UNIVERSIDADE DE BRASÍLIA

INSTITUTO DE CIÊNCIAS BIOLÓGICAS

PROGRAMA DE PÓS-GRADUAÇÃO EM ECOLOGIA

\title{
PRIMATAS DO CERRADO: CONSERVAÇÃO, BIOGEOGRAFIA E MUDANÇAS CLIMÁTICAS
}

\author{
Danilo Gustavo Rodrigues de Oliveira \\ Orientador: Prof. Dr. Ricardo Bomfim Machado \\ Co-orientador: Prof. Dr. Fabiano Rodrigues de Melo
}

Brasília, agosto de 2015 
UNIVERSIDADE DE BRASÍLIA

INSTITUTO DE CIÊNCIAS BIOLÓGICAS

PROGRAMA DE PÓS-GRADUAÇÃO EM ECOLOGIA

\title{
PRIMATAS DO CERRADO: CONSERVAÇÃO, BIOGEOGRAFIA E MUDANÇAS CLIMÁTICAS
}

\author{
Orientador: Dr. Ricardo B. Machado \\ Tese apresentada ao Programa de \\ Pós-Graduação em Ecologia, \\ Instituto de Ciências Biológicas da \\ Universidade de Brasília, como \\ parte dos requisitos necessários \\ para a obtenção do título de Doutor \\ em Ecologia
}

Brasília-DF, 2015 


\section{AGRADECIMENTOS}

Agradeço primeiramente a Deus que é sempre um porto-seguro em minha vida e me dá forças e esperança a cada dia para continuar.

Agradeço aos meus pais José Gilnei e Maria das Mercês, que sempre me incentivaram a estudar e me apoiaram em todos os momentos. Sem o apoio deles nada disso seria possível. Tenho muito a agradecer à minha esposa Thaís Imperatori que foi compreensiva e amorosa em todos os momentos, especialmente nos de dificuldade e ausência, te amo.

Agradeço este trabalho ao meu orientador Ricardo "Pacheco" Machado, pela orientação, liberdade e paciência durante todo este tempo. O apoio e as discussões foram fundamentais para a minha formação acadêmica. Agradeço também ao prof. Fabiano de Melo que me auxiliou na montagem da base de dados, em expedições de campo e com várias correções e ensinamentos científicos. Muito obrigado!!

Aos amigos do Laboratório de Planejamento para Conservação da Biodiversidade (LaBIO) que me ensinaram muito e me apoiaram em minhas dúvidas e angústias. Obrigado Vivian, Thallita, Renatinha, Tatá, Giovanna, Yuri, Babi, Mariana, Romina, Reile, Fernando Goulart e Diego.

Aos amigos do Programa de Pós-Graduação em Ecologia que compartilharam tanto ideias e frustrações como risadas e descontrações. Muito obrigado a todos!! Também agradeço ao programa de pós-graduação em Ecologia por possibilitar o desenvolvimento desta pesquisa. 
Agradeço aos doutores Carlos Abs da Cruz Bianchi, Francisco Dyonísio Cardoso Mendes, Maria Clotilde Henrique Tavares, Regina Helena Ferraz Macedo e André Faria Mendonça por terem aceitado participar da banca e pelas sugestões.

Aos funcionários e professores do IB, pelo bom funcionamento do Instituto e pela qualidade das disciplinas ofertadas e do trabalho realizado.

A todos que contribuíram de alguma forma, meu muito obrigado!! 


\section{SUMÁRIO}

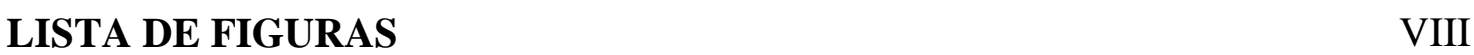

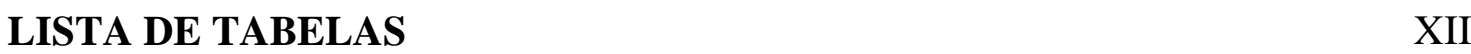

$\begin{array}{ll}\text { RESUMO GERAL XIV } & \text { XIV }\end{array}$

CAPITULO 1 - Introdução Geral

INTRODUÇÃO GERAL 2

$\begin{array}{lr}\text { Primatas do Cerrado } & 4\end{array}$

$\begin{array}{ll}\text { Mudanças Climáticas } & 7\end{array}$

$\begin{array}{lr}\text { Modelagem de Nicho Ecológico } & 9\end{array}$

$\begin{array}{lr}\text { APRESENTAÇÃO DA TESE } & 12\end{array}$

REFERÊNCIAS BIBLIOGRÁFICAS

CAPITULO 2- Prioridades para a conservação de primatas do Cerrado e identificação de lacunas de Conservação

RESUMO

$\begin{array}{lr}\text { ABSTRACT } & 19\end{array}$

$\begin{array}{ll}\text { INTRODUÇÃO } & 20\end{array}$

MÉTODOS 23

$\begin{array}{ll}\text { Dados das Espécies } & 23\end{array}$

Modelagem de Nicho Ecológico 25

$\begin{array}{lr}\text { Lacunas de Conservação } & 28\end{array}$

Prioridades de Conservação $\quad 29$

$\begin{array}{ll}\text { RESULTADOS } & 30\end{array}$

$\begin{array}{ll}\text { Modelos de Nicho } & 30\end{array}$

$\begin{array}{ll}\text { Prioridades de Conservação } & 33\end{array}$ 
$\begin{array}{ll}\text { DISCUSSÃO } & 35\end{array}$

Variáveis preditoras da presença de primatas 35

$\begin{array}{ll}\text { Grau de ameaça } & 37\end{array}$

$\begin{array}{ll}\text { Modelos x Pontos } & 37\end{array}$

Priorização de áreas para conservação $\quad 39$

$\begin{array}{ll}\text { Conclusões } & 41\end{array}$

$\begin{array}{ll}\text { REFERENCIAS } & 42\end{array}$

CAPITULO 3 - Áreas de estabilidade climática e de endemismo para Primatas no Cerrado

$\begin{array}{ll}\text { RESUMO } & 56\end{array}$

$\begin{array}{ll}\text { ABSTRACT } & 56\end{array}$

$\begin{array}{ll}\text { INTRODUÇÃO } & 57\end{array}$

MATERIAL E MÉTODOS $\quad 60$

$\begin{array}{ll}\text { Dados das espécies } & 60\end{array}$

Modelagem de Nicho Ecológico $\quad 62$

$\begin{array}{ll}\text { Áreas de endemismo e vicariância } & 64\end{array}$

$\begin{array}{ll}\text { RESULTADOS } & 66\end{array}$

$\begin{array}{ll}\text { DISCUSSÃO } & 76\end{array}$

$\begin{array}{ll}\text { Áreas de endemismo } & 76\end{array}$

$\begin{array}{ll}\text { Refúgios de estabilidade climática } & 79\end{array}$

$\begin{array}{lr}\text { Cerrado e biomas vizinhos } & 82\end{array}$

$\begin{array}{lr}\text { Considerações finais } & 84\end{array}$

REFERÊNCIAS BIBLIOGRÁFICAS 85

CAPITULO 4- Efeito das mudanças climáticas e fragmentação do habitat 90 
sobre a primatofauna do Cerrado

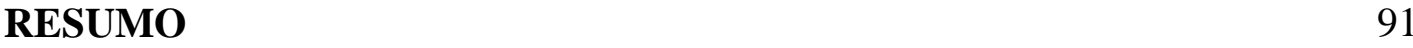

$\begin{array}{ll}\text { ABSTRACT } & 91\end{array}$

$\begin{array}{ll}\text { INTRODUÇÃO } & 92\end{array}$

MATERIAL E MÉTODOS 95

Modelagem de Nicho Ecológico $\quad 95$

Grau de ameaça de extinção no futuro 97

$\begin{array}{lr}\text { Análises estatísticas } & 99\end{array}$

$\begin{array}{lr}\text { RESULTADOS } & 100\end{array}$

$\begin{array}{lr}\text { DISCUSSÃO } & 110\end{array}$

Cenários climáticos 110

Uso da terra e deslocamento na paisagem 112

$\begin{array}{ll}\text { Graus de ameaça } & 114\end{array}$

$\begin{array}{lr}\text { REFERÊNCIAS BIBLIOGRÁFICAS } & 118\end{array}$

CAPITULO 5- Conclusões gerais 130

$\begin{array}{ll}\text { Conclusões } & 131\end{array}$

Limitações do Estudo 133

Implicações para Conservação 135

Referências Bibliográficas 136

$\begin{array}{ll}\text { ANEXOS } & 137\end{array}$

Anexo 1- Diferenças entre pares de projeções para o passado 138

Anexo 2- Remanescentes nativos no cerrado para o presente e estimados para 2050

Anexo 3- Projeções para o futuro nos quatro diferentes modelos globais de circulação atmosfera-oceano e cenários climáticos 
Anexo 4- Lista das localidades de registros de primatas do Cerrado com suas coordenadas geográficas

Anexo 5- Lista das instituições e referências que possuem registros de primatas do cerrado 


\section{LISTA DE FIGURAS}

\section{CAPITULO 1}

Figura 1. Representação dos principais tipos de vegetação do Cerrado. A transição savana-floresta representa um mosaico com manchas de florestas e savanas. (Adaptado de Silva e Bates, 2002)

Figura 2. Mapa das áreas de endemismo dos primatas neotropicais feito com análise parcimônica de endemismo com quadrantes de $5^{\circ} \times 5^{\circ}$, adaptado de Goldani et al. (2006). A área em verde representa a área do Cerrado conforme proposta do IBGE (2004)

Figura 3. Mudanças projetadas nos cenários RCP2.6, 4.5, 6.0 e 8.5 do IPCC para a temperatura média global (a), as emissões de $\mathrm{CO}_{2}$ (b), as emissões de $\mathrm{CH}_{4}$ (c), as emissões de $\mathrm{NO}_{2}$ (d) e as emissões de $\mathrm{SO}_{2}$ (e). (Modificado de: IPCC 2014).

\section{CAPÍTULO 2}

Figura 1. A extensão do Cerrado mostrado as áreas antropizadas em cinza e remanescentes de vegetação nativa em verde até o ano de 2010, segundo o 50 IBAMA (MMA/IBAMA 2011b).

Figura 2. Mapas binários de adequabilidade ambiental para 15 taxa de primatas

Figura 3. Número de táxa em cada célula baseado em modelos de nicho para 15 primatas. 
Figura 4. Mapas de áreas prioritárias para conservação de primatas no Cerrado gerados utilizando pontos de ocorrência (A) e modelos de nicho (B). Áreas vermelhas correspondem às áreas prioritárias.

Figura 5. Mapas de insubstituibilidade considerando pontos de ocorrência (A) e modelos de distribuição (B), considerando as reservas já existentes (em verde). Valor obtido utilizando as 1000 soluções de priorização.

\section{CAPÍTULO 3}

Figura 1. Mapas de riqueza das espécies de primatas do Cerrado no presente (A), holoceno médio (6 mil anos) (B), última glaciação (21 mil anos) (C) e último período inter-glacial (130 mil anos) (D).

Figura 2. Soma das adequabilidades climáticas históricas obtido pela soma das projeções dos cenários do presente, 6 mil, 21 mil e 130 mil anos para cada espécie e calculando a média para cada uma. Valores mais altos indicam áreas que foram selecionadas como presença em maior número de cenários.

Figura 3. Foco na distribuição potencial histórica de Alouatta belzebul mostrando as mudanças nas duas áreas de distribuição disjunta ao longo do tempo.

Figura 4. Áreas de endemismo geradas com o método de otimização NDM com tamanho de célula de $2^{\circ} \times 2^{\circ}(\mathrm{A} \mathrm{e} \mathrm{B}), 3^{\circ} \times 3^{\circ}\left(\mathrm{C}, \mathrm{D}\right.$ e E) e $4^{\circ} \times 4^{\circ}(\mathrm{F}, \mathrm{G} \mathrm{e} \mathrm{H})$. Áreas similares foram fundidas em um consenso das espécies presentes na área.

Figura 5. Áreas de endemismo geradas com o programa NDM utilizando pontos de ocorrência e modelos de distribuição de espécies. Valores 
correspondem ao índice de endemicidade.

Figura 6. Mapa mostrando a delimitação das áreas de endemismo baseado na distribuição de primatas que habitam o Cerrado. (A) Transição leste, (B) Serras do Centro-Leste, (C) Transição norte e (D) Transição oeste.

Figura 7. Mapas de estabilidade climática histórica média ao longo do tempo considerando somente as espécies que contribuem para a delimitação das áreas de endemismo calculadas na Transição leste (A), Serras do Centro-Leste (B), Transição norte (C) e Transição oeste (D).

\section{CAPÍTULO 4}

Figura 1. Diagrama explicativo de como foram gerados os fatores de condição de deslocamento possível para as espécies.

Figura 2. Figura representando a adequabilidade climática média (cor) para os primatas do Cerrado no presente (esquerda, no alto) e nos anos de 2050 (esquerda) e 2080 (direita) para os cenários otimista (RCP 26 no centro) e pessimista (RCP 85 abaixo).

Figura 3. Mudança na riqueza de espécies ('turnover') existente entre o presente e o ano de 2050 (A e C) e o ano de 2080 (B e D), nos cenários otimista (A e B) e pessimista (C e D). Azul indica que a área não perderá riqueza no futuro, enquanto amarelo e vermelho indicam a quantidade de espécies que cada célula perderá.

Figura 4. Gráficos das diferenças de áreas projetadas nos diferentes cenários 
climáticos (presente, otimista 2050, otimista 2080, negativo 2050 e negativo 2080) e nas diferentes condições de deslocamento. Na parte de cima, gráfico com a área em todo o mapa e na parte de baixo somente no Cerrado.

Figura 5. Análises pareadas mostrando diferenças significativas entre os pares de projeções presente e negativo 2050 para cada espécie. Cores representam com qual cenário a célula está mais semelhante: quanto mais próximo do verde mais parecido somente com o futuro e mais próximo do branco significa só semelhante ao presente. Verde escuro representa diferença significativa do presente, áreas em branco são diferentes do futuro, áreas em rosa não demonstram diferença entre presente e futuro e áreas em bege não são ocupadas.

\section{ANEXOS}

Figura A.1. Figuras ilustrando as diferenças entre pares de projeções temporais para o passado. Áreas em verde representam áreas potencialmente ocupadas no período mais recente, áreas em vermelho representam áreas que deixaram de ser potencialmente ocupadas e áreas em amarelo não modificaram seu valor entre os períodos de tempo.

Figura A.2. Áreas de remanescentes nativos no Cerrado (em verde) no presente e estimados para o ano de 2050.

Figura A.3. Figuras representando as projeções de adequabilidade climática para as espécies de primatas analisadas nos diferentes modelos AOGCM e cenários climáticos. 


\section{LISTA DE TABELAS}

\section{CAPÍTULO 2}

Tabela 1. Valores de densidade populacional e metas de conservação para os gêneros de primatas estudados.

Tabela 2. Valor médio da importância de cada variável para a previsão da adequabilidade climática gerada pelos modelos. Quanto maior o valor, maior a importância da variável. Os números nas colunas representam cada um dos taxa usados.

Tabela 3. Quantidade de área que já foi desmatada até 2010 da área de ocorrência esperada $\left(\mathrm{em} \mathrm{km}^{2}\right)$ das espécies de primatas no Cerrado.

Tabela 4. Efetividade do atual sistema de reservas do Cerrado para a proteção de primatas.

\section{CAPÍTULO 3}

Tabela 1. Valores da estatística $\mathrm{T}$ para agrupamento da distribuição das espécies calculado com um modelo nulo.

Tabela 2. Resultados da análise de endemicidade baseada em pontos.

Tabela 3. Resultados da análise de endemicidade baseada em pontos e modelos.

\section{CAPÍTULO 4}


Tabela 1. Valores das áreas (em milhares de $\mathrm{km}^{2}$ ) e dos percentuais de perdas de área projetadas para as espécies do Cerrado nos anos de 2050 e 2080 e nos cenários otimista e pessimista. Foi considerado que os táxons não terão liberdade para migrar para locais em que eles não estavam no presente.

Tabela 2. Graus de ameaça de extinção no presente e no ano de 2050 para as condições sem migração, somente no Cerrado e de desmatamento no Cerrado. As letras correspondem aos níveis de ameaça: menor preocupação (LC), quase ameaçado (NT), vulnerável (VU), em perigo (EN) e criticamente em perigo (CR). Espécies em negrito possuem risco crítico em mais de uma condição.

\section{ANEXOS}

Tabela A.1. Número de registros de ocorrência por espécie de primata 


\section{RESUMO GERAL}

Os primatas neotropicais dependem de ambientes com estrato arbóreo para viver. Essa dependência os deixa particularmente vulneráveis às mudanças no uso da terra para fins antrópicos. O Cerrado é o segundo maior bioma do Brasil e tem apresentado uma alta taxa de desmatamento nos últimos anos, devido ao avanço da agricultura e pecuária na região. Além disso, o clima tem passado por fortes mudanças que alteraram os seus limites e devem alterar a distribuição de várias espécies nos próximos anos. O objetivo desta tese foi analisar os padrões biogeográficos históricos, atuais e futuros dos primatas do Cerrado e as implicações para a conservação do grupo. Objetivos específicos incluem: analisar a eficiência do atual sistema de áreas protegidas do Cerrado para a proteção de primatas; apontar áreas prioritárias para conservação que complementam as já existentes; apontar áreas de endemismo; apontar zonas de estabilidade climática; compreender a contribuição de biomas vizinhos para a primatofauna da região; verificar o efeito das mudanças climáticas e do uso da terra na distribuição de primatas; comparar a magnitude do efeito das mudanças no futuro; e projetar mudanças futuras para o grau de ameaça. As ocorrências de 16 taxóns de primatas foram coletadas a partir de coleções em museus e literatura científica. Foram utilizados modelos de nicho ecológico no pacote biomod2 do software $\mathrm{R}$ em uma abordagem de consenso de algoritmos para estimar os requisitos de nicho das espécies. Foram utilizadas as variáveis bioclimáticas como variáveis ambientais e também foram utilizadas três variáveis obtidas de imagens de satélite para descrever a vegetação no período presente. Os modelos de nicho foram projetados para os períodos: 130 mil aap. (anos antes do presente), 21 mil aap., 6 mil aap., presente, ano de 2050 e ano de 2080. As projeções para o futuro foram realizadas com o cenário mais otimista e o mais pessimista, RCP2.6 e RCP8.5 respectivamente. Foi feita a priorização de áreas para conservação com o programa Marxan e a análise de lacunas de conservação com o ArcMap 10.2. As áreas de endemismo foram identificadas com o programa NDM usando pontos e pontos mais modelos. O agrupamento da distribuição das espécies foi testada usando o teste $\mathrm{T}$ do pacote prabclus. As zonas de estabilidade climática foram determinadas como áreas que se mantiveram adequadas para a permanência da espécie no passado por mais tempo. A mudança no uso da terra foi estimada para o ano de 2050 
com a ferramenta LCM do programa IDRISI. Foram utilizados os critérios A e B da União Internacional para a Conservação da Natureza (IUCN) para estimar o grau de ameaça projetado para primatas. De acordo com a estimativa realizada, as principais características do meio que afetam a distribuição de primatas do Cerrado são a precipitação média anual e a sazonalidade da temperatura. Um terço dos taxóns analisados constituem lacunas reais de conservação e apenas um táxon é uma lacuna potencial (estimada por modelos). As áreas prioritárias apontadas foram, em geral, maiores e mais contínuas no norte do Cerrado e mais fragmentadas e isoladas no sul, devido ao maior grau de antropização do sul do domínio. A distribuição dos primatas não é randômica, o que indica existência de uma força que possivelmente moldou a distribuição do grupo no passado. O consenso das áreas de endemismo apontou agrupamento de espécies na transição leste, oeste e norte do domínio e também nas serras do centro-leste do Brasil. As zonas de estabilidade climática médias se distribuem em uma faixa no sul do Cerrado que atravessa do Chaco à Mata Atlântica e são mais concentradas em áreas de alta altitude em espécies das áreas de endemismo do leste do domínio e em baixas altitudes para espécies das áreas oeste e norte. No futuro, a área de ocorrência das espécies deve reduzir, em média, de 9,3\% a 59,2\% dependendo da sua capacidade de migração. Dentro do Cerrado, a perda de área média das espécies fica em torno de $84,4 \%$ quando consideramos a expectativa de remanescentes nativos para 2050. Seis táxons irão aumentar o grau de ameaça e quatro correm risco de serem extintos do Cerrado. Comparando as projeções para o passado, presente e futuro emerge a importância da conservação de espécies que habitam áreas marginais no leste e oeste do domínio. 
CAPÍTULO 1

Introdução geral 


\section{INTRODUÇÃO GERAL}

O Cerrado é uma formação savânica que ocupa boa parte do Brasil central e faz contato com quase todos os demais biomas brasileiros. A área ocupada pelo bioma é de aproximadamente $2.000 .000 \mathrm{~km}^{2}$, o que representa cerca de $23 \%$ do território brasileiro (IBGE 2004). O clima da região é classificado como Aw na classificação de Köppen (tropical chuvoso), com duas estações bem definidas: uma seca (de maio a setembro) e outra chuvosa (de outubro a abril) (Eiten 1972). A média de precipitação anual é igual a 1500 mm, variando de 750 a 2000 mm (Eiten 1972, 1994).

O Cerrado é constituído por um mosaico de formações vegetais de diferentes formas e características. Ribeiro e Walter (1998) dividem o Cerrado em onze fitofisionomias diferentes, sendo que quatro delas são formações florestais. As savanas ocupam aproximadamente $72 \%$ da área do Cerrado e as áreas de floresta ou transição savana-floresta representam um total de $28 \%$ da área do bioma (Silva and Bates 2002) (Figura 1).

Myers e colaboradores (Myers et al. 2000), classificaram os biomas com grande concentração de endemismos e alto grau de alteração ambiental como hotspots para a conservação da biodiversidade e o Cerrado está na lista dos 32 hotspots (Mittermeier et al., 2005), pela alta diversidade de plantas . Estima-se que nos últimos 35 anos mais da metade da área original do Cerrado tenha sido convertida para pastagem, lavoura e outros usos e apenas $2,2 \%$ da área do bioma está protegida para conservação ambiental (Klink and Machado 2005). Se o ritmo de desmatamento se mantiver igual ao ocorrido no passado nos próximos anos o Cerrado será totalmente convertido para usos antrópicos ate 2030 (Machado et al. 2004). 


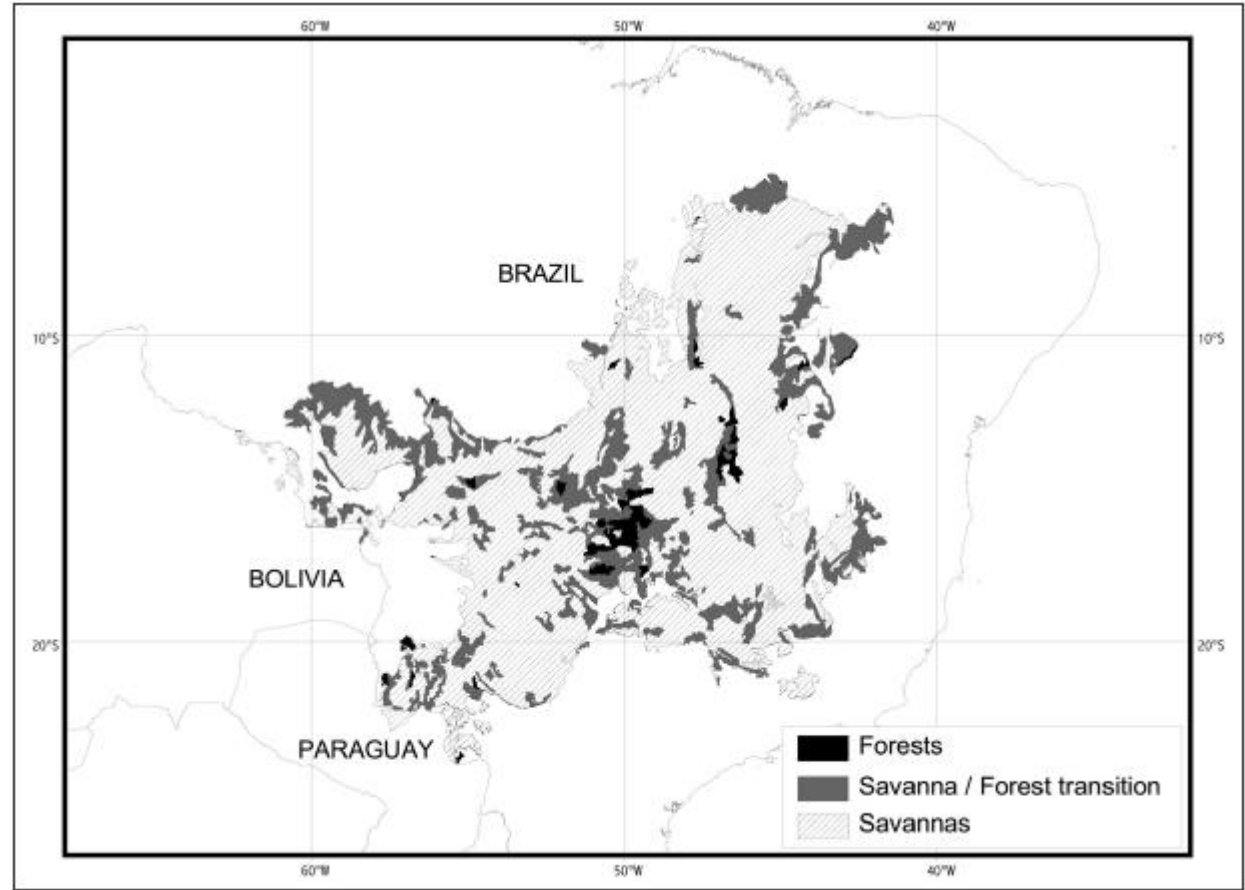

Figura 1. Representação dos principais tipos de vegetação do Cerrado. A transição savana-floresta representa um mosaico com manchas de florestas e savanas. (Adaptado de Silva e Bates, 2002).

A biodiversidade do Cerrado apresenta uma riqueza de espécies expressiva (Machado et al. 2008), atingindo uma riqueza de angiospermas estimada em 10.000 espécies (Ratter et al. 1997). A fauna também apresenta grande riqueza, com aproximadamente 177 espécies de répteis (Aguiar et al. 2004), 856 de aves (Silva and Santos, 2005) e 251 de mamíferos (Paglia et al. 2012), dentre os quais 16 de primatas (Hisch et al., 2002). Apesar de ser um bioma constituído em sua maioria por formações savânicas e campestres, $82 \%$ das aves e de 50 a $80 \%$ dos mamíferos possuem algum grau de dependência de ambientes florestais (Redford and Fonseca 1986, Silva 1995, Aguiar et al. 2004). As populações de primatas brasileiros necessitam de ambientes com estrato arbóreo e podem utilizar essas áreas para viver e reproduzir ou como corredores entre duas manchas maiores. Elas podem ainda utilizar ou ter utilizado essas manchas florestais como rotas de dispersão entre biomas diferentes, já que foi identificado grande número de espécies arbóreas que ocorrem desde a Floresta Amazônica até a Atlântica 
atravessando o Cerrado pelos cursos d'água na diagonal noroeste sudeste (OliveiraFilho and Ratter 1995).

\section{Primatas do Cerrado}

A ordem primates se distribui principalmente em regiões tropicais na África, Madagascar, Ásia e Neotrópicos, com uma diversidade de 348 espécies em 18 famílias (Lehman and Fleagle, 2006). A ordem se divide em Strepsirrhini, que habitam a ilha de Madagascar, e Haplorrhini, que se subdivide em Catarrhini e Platyrrhini. Os Catarrhini ocupam a África e a Ásia e são conhecidos como primatas do Velho Mundo, enquanto Platyrrhini ocupa a região Neotropical e são conhecidos como primatas do Novo Mundo (Fleagle, 1999). O Brasil possui 118 táxons de primatas distribuídos em cinco famílias: Callithrichidae, Cebidae, Aotidae, Pithecidae e Atelidae (Paglia et al., 2012)

Primatas são animais predominantemente arborícolas e excluindo alguns gêneros de macacos do Velho Mundo, como Pan, Papio e Macaca, todos os demais são dependentes de estrato arbóreo para se locomover e se alimentar (Fleagle 1999). Todos os primatas neotropicais habitam ambientes com variados graus de arborização. Em uma floresta alta, como a amazônica, as espécies podem se especializar em diferentes habitats na floresta, inclusive em diferentes estratos da vegetação (Warner 2002; Schwarzkopf \& Rylands 1989), aumentando a densidade demográfica local. Porém em formações vegetais mais baixas, como as do Cerrado, a diversidade de habitats é menor e os grupos de primatas precisam competir de forma mais intensa para manter o seu território (Lazaro-Perea 2001; Decanini \& Macedo 2008)

A riqueza de primatas possui uma associação muito característica com a precipitação da região. Até $2500 \mathrm{~mm}$ de precipitação anual a riqueza de primatas aumenta com o aumento da precipitação e depois desse limite há um decréscimo da riqueza de primatas (Kay et al. 1997). Esse trabalho sugere que a riqueza decresce após 
2500mm de precipitação devido à queda da produtividade primária das plantas e que o principal fator que determina a riqueza de primatas neotropicais é a produtividade primária. A produtividade primária afeta diretamente a capacidade das plantas de produzir itens alimentares, o que também é um fator que afeta a riqueza do grupo (Stevenson 2001; Hanya et al. 2011).

Uma revisão das ocorrências de primatas neotropicais (Hirsch et al. 2002) apontou que 15 táxons de primatas ocorrem no Cerrado, sendo elas: Alouatta caraya, A. guariba, A. belzebul, Aotus azarae azarae, A. a. infulatus, Callicebus nigrifons, C. personatus, Callithrix geoffroyi, C. jacchus, C. penicillata, Mico melanurus, Sapajus cay, S. libidinosus, S. nigritus e S. xanthosternos. No caso de alguns primatas do Cerrado, observa-se o desenvolvimento de adaptações para sobreviver na época da seca, quando a disponibilidade de alimentos é menor. Por exemplo, animais do gênero Callithrix possuem dentes adaptados para escavar buracos (escarificar) na casca das árvores e retirar exsudatos vegetais para se alimentar. Na época da seca, a proporção dos exsudatos vegetais na dieta desses animais é maior e na época chuvosa aumenta o consumo de frutos (Vilela \& Faria 2002). Em São Paulo, os macacos-prego aumentam o consumo de sementes e flores na época de seca e no caso de bugios existe um aumento na quantidade de folhas na dieta (Rodrigues, 1992). Sapajus libidinosus pode complementar a dieta com itens obtidos das populações humanas que frequentam a área em que moram (Sacramento 2014)

Os biomas sofreram ao longo dos anos contrações e expansões como resultado de mudanças climáticas pronunciadas, como as eras glaciais. Haffer (1969), sugeriu que a savanização de várias áreas da Amazônia forçou as espécies de aves a migrarem para regiões que se mantiveram florestadas e esses refúgios florestais teriam agido como centros de especiação e de dispersão de espécies. Kinzey (1982), analisou a existência 
de alguns refúgios florestais para primatas na Mata Atlântica e sugeriu três centros de endemismos diferentes dos obtidos com evidências herpetológicas. Jones (1987), relata observações que reforçam a ideia que os refúgios florestais propostos para aves na África são semelhantes aos de primatas. (Silva \& Oren 1996), utilizaram os dados sobre as comunidades de primatas na Amazônia em uma análise parcimônica de endemismo (PAE) e viram que os centros de endemismos coincidem com os centros propostos para aves na mesma região. Silva (1997), propôs três áreas de endemismo no Cerrado para aves e ainda não foi analisado qual é a relevância dessas áreas para os primatas. Segundo Cracraft (1994), áreas de endemismo similares sugerem estruturações históricas e biogeográficas similares nos clados por processos de vicariância.

Goldani et al. (2006), analisaram a distribuição das espécies de primatas neotropicais e fizeram uma análise parsimônica de endemismo (PAE, método para reconstruir áreas de endemismo) buscando regiões com padrões filogenéticos de primatas parecidos. Eles encontraram diversas áreas de endemismo diferentes, que estão representadas na figura 2. A principal área de endemismo do Cerrado (A6) ocupa também boa parte da Caatinga. No entanto, a fauna de primatas do Cerrado também tem relação com áreas de endemismo típicas da Amazônia (A2), Mata Atlântica (A4) e Pantanal e Chaco (A5). O trabalho de Goldani et al. (2006) apresenta a PAE de três formas diferentes e concluiu que a análise tende a agrupar a fauna do Cerrado, Caatinga, Mata Atlântica e Chaco. Isso condiz com o fato de a flora da Mata Atlântica ser mais similar à do Cerrado que a flora da Amazônia (Méio et al. 2003). 


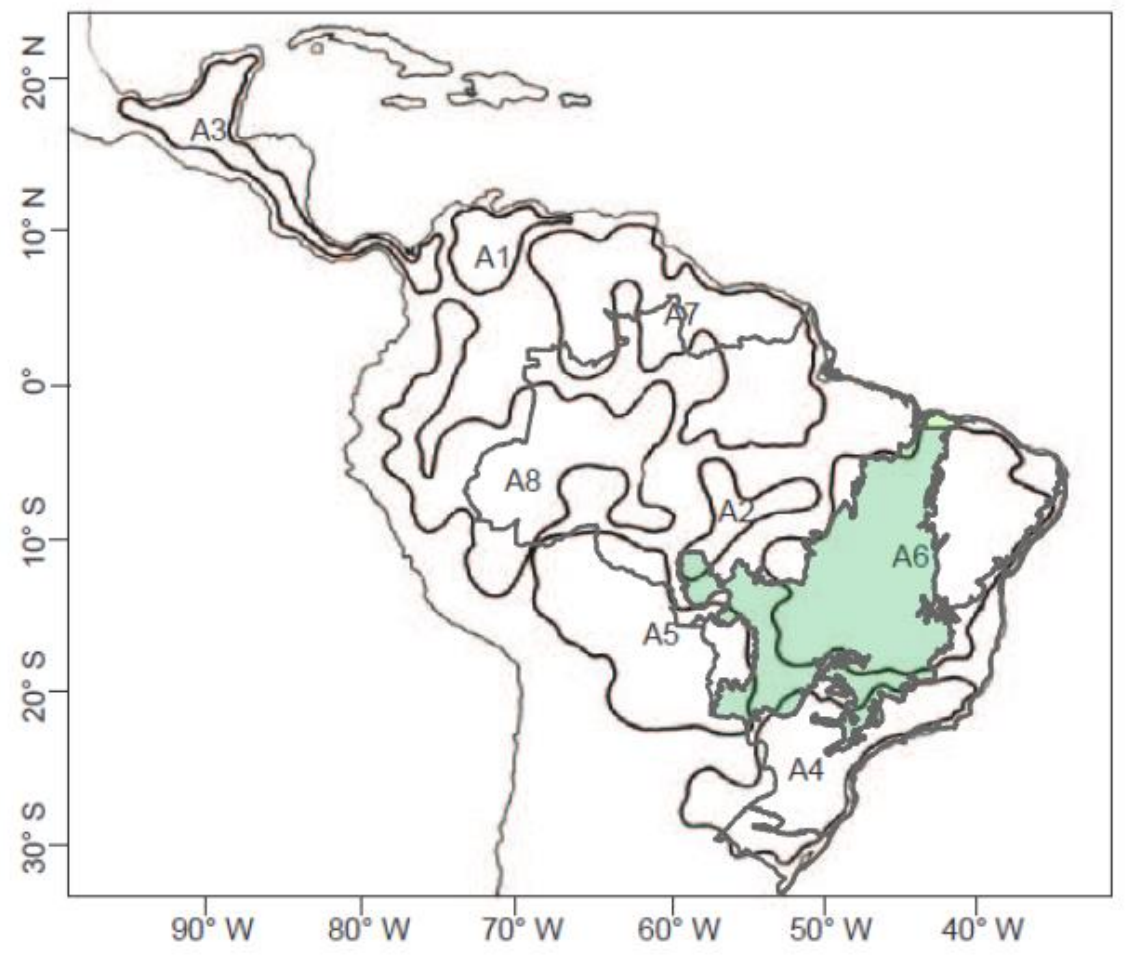

Figura 2. Mapa das áreas de endemismo dos primatas neotropicais feito com análise parcimônica de endemismo com quadrantes de $5^{\circ} \times 5^{\circ}$, os códigos de A1 a A8 representam as diferentes áreas de endemismo apontadas (adaptado de: Goldani et al. 2006). A área em verde representa a área do Cerrado conforme proposta do IBGE (2004).

\section{Mudanças climáticas}

O ambiente do Cerrado variou muito de clima nos últimos milhões de anos e passou por pelo menos cinco ciclos de resfriamento e aquecimento durante o período Quaternário (Salgado-Labouriau 1994). As flutuações climáticas do quaternário fizeram com que as fitofisionomias florestais se expandissem nos períodos mais quentes e úmidos (interglaciais) e se retraíssem nos períodos mais frios e secos (glaciais), aumentando a proporção das formações abertas (Bigarella and Andrade-Lima 1982). Essas flutuações do clima e das formações vegetais associadas a ele tiveram um grande impacto na fauna da região, de forma que muitos grupos animais tiveram que encontrar 
um local para se refugiar em um período climaticamente desfavorável (Haffer 1969, Kinzey 1982, Prance 1982).

As flutuações climáticas ao longo dos últimos milhares de anos são explicadas por mudanças na distância do sol para a Terra ao longo dos anos por causa de pequenas variações na órbita da Terra (Bigarella and Andrade-Lima 1982). No entanto, o mundo está atualmente passando por um período de mudanças climáticas provocadas pela atividade humana. Esse aumento global da temperatura ocorre por causa do aumento das emissões de gases do efeito estufa, devido à queima de combustíveis fósseis, às mudanças no uso da terra e atividades industriais (IPCC 2014). Os gases do efeito estufa permitem a entrada de calor, porém dificultam a saída dele da atmosfera, portanto sua maior concentração na atmosfera causa um represamento do calor e aumento da temperatura (Chapin et al. 2002).

O Painel Intergovernamental Para as Mudanças Climáticas (IPCC) da ONU fez projeções de como mudará o clima do planeta nos próximos anos baseado em cenários de emissão de gases do efeito estufa, chamados de caminhos representativos de concentração (RCP). Os cenários modelados no IPCC são quatro: o RCP2.6 projeta um aumento dos gases do efeito estufa até o ano de 2020 e depois uma redução; o RCP4.5 estima o aumento dos gases até o ano de 2040, com o posterior declínio; o RCP6.0 prevê que as emissões terão um pico no ano de 2080 e o RCP8.5 prevê que as emissões continuam a aumentar ao longo do século XXI (IPCC 2014) (Figura 3). A temperatura tende a aumentar de 0,3 a 1,7 graus celsius no RCP2.6 e de 2,6 a 4,8 no RCP8.5 até o ano de 2100 (IPCC 2014). 
(a) Média global de mudança de temperatura

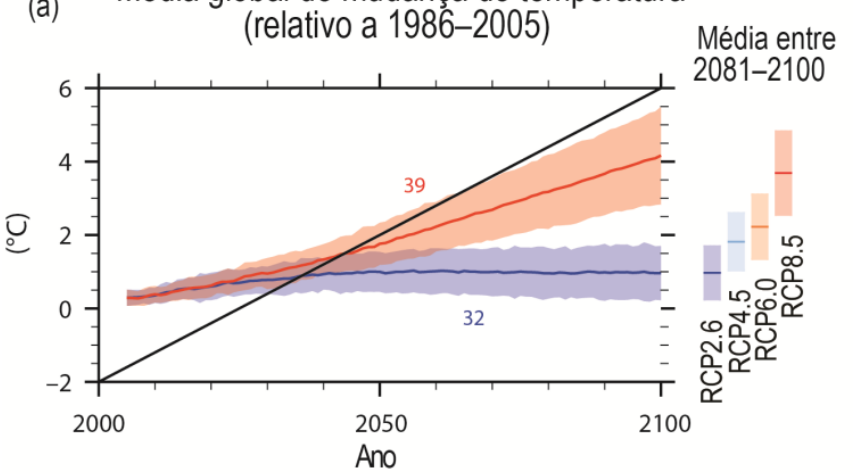

- Emissões

históricas

Cenários RCP

- RCP8.5

- RCP6.0

- RCP4.5

$-\mathrm{RCP} 2.6$

T Faixa total de variação

$\perp$ dos cenários em 2010
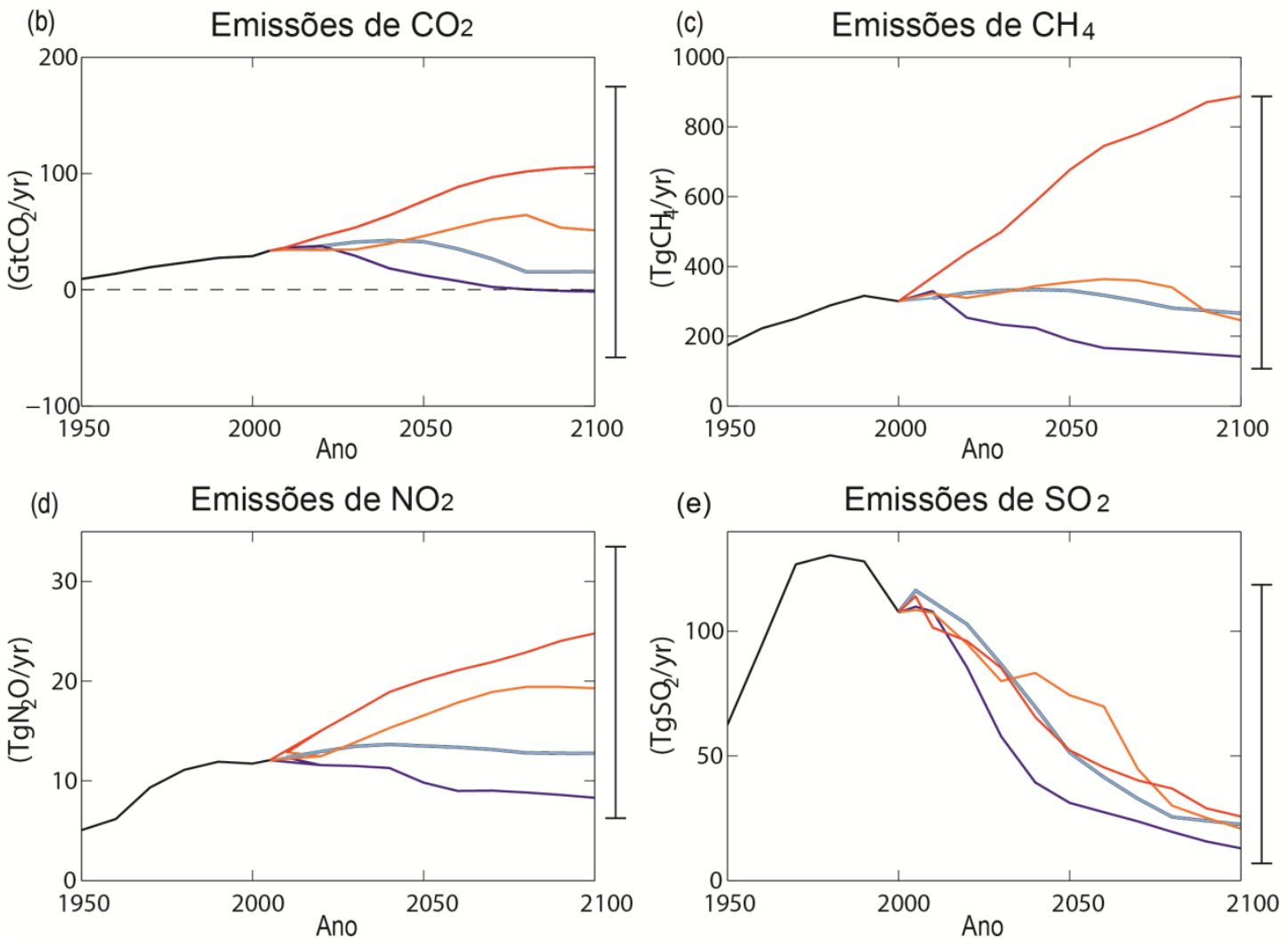

Figura 3. Mudanças projetadas nos cenários RCP2.6, 4.5, 6.0 e 8.5 do IPCC para a temperatura média global (a), as emissões de $\mathrm{CO}_{2}$ (b), as emissões de $\mathrm{CH}_{4}$ (c), as emissões de $\mathrm{NO}_{2}$ (d) e as emissões de $\mathrm{SO}_{2}$ (e). (Modificado de: IPCC 2014).

\section{Modelagem de nicho ecológico}

Uma boa ferramenta para se compreender possíveis mudanças na distribuição das espécies causadas por variações no clima é a modelagem de nicho ecológico, porque projeta resposta de distribuição das espécies frente ao clima (Peterson et al., 2011). Para definirmos o que é esse método faz-se necessário primeiramente deixar claro qual é o 
conceito de nicho utilizado para fins do presente estudo. Apesar de amplamente utilizado e disseminado em toda a ecologia a definição exata de nicho é difícil de concretizar. O primeiro conceito de nicho foi apresentado por Joseph Grinnell, que sugeriu que nicho é a área que o organismo ocupa no seu habitat (Grinnell 1917). Isto implica que as espécies ocupam um local no ambiente com condições abióticas favoráveis. Outro famoso conceito de nicho foi utilizado por Charles Elton se referindo à função ou ao papel da espécie (Elton 1962). Este se relaciona mais à posição da espécie em cadeias tróficas e em outras formas de espaços de interação biótica (Polechová \& Storch 2008).

Estes duas formas de entender nichodominaram a discussão sobre a teoria de nichos até o final da década de 50, quando surgiu a teoria unificadora de George E. Hutchinson (Hutchinson 1958). Segundo este autor, nicho é composto por um 'hipervolume' em um espaço ecológico multidimensional determinado pelas necessidades da espécie para reproduzir e sobreviver. Esse conceito considera que todos os fatores bióticos (também chamados de variáveis recurso ou bionômicas) e abióticos (também chamadas de variáveis condição ou cenopoéticas) agem em conjunto para delimitar o nicho da espécie (Soberón 2007). Hutchinson também definiu nicho fundamental, que é um espaço multidimensional (variáveis bionômicas e cenopoéticas) em que a espécie consegue reproduzir e sobreviver indefinidamente, e nicho realizado, que é uma parte do nicho fundamental em que a espécie consegue permanecer mesmo em competição com outras (Polechová \& Storch 2008).

Ao se realizar uma modelagem de nicho ecológico fazemos uso da grande quantidade de informação que se tornou disponível em uma escala global sobre o meio abiótico. A dinâmica e estrutura das variáveis bionômicas são mais localizadas e difíceis de extrapolar para uma escala global (Soberón 2007). Portanto, o conceito de nicho 
utilizado é o de nicho Grinnelliano (conforme Grinnel), seguindo a visão de espaço multidimensional estabelecida por Hutchinson.

A ferramenta de modelagem ecológica utiliza variáveis ambientais e climáticas do lugar onde se registrou a ocorrência das espécies para construir um modelo do nicho utilizado por elas em todos os pontos de ocorrência conhecidos. A partir desse modelo, ela projeta as condições ambientais propícias para a ocorrência das espécies para locais em que a ocorrência não é conhecida e gera um mapa de distribuição potencial ao longo da área considerada. A partir das variáveis analisadas no treino do modelo, a análise também pode extrapolar a ocorrência das espécies em outros períodos de tempo ou em outras regiões geográficas.

Atualmente, existem dezenas de modelos diferentes para prever a ocorrência de espécies e não existe um consenso na literatura sobre o melhor deles (Elith et. al., 2006; Qiao et. al., 2015). Vários estudos foram realizados para se encontrar o modelo que consegue prever a ocorrência de espécies com maior precisão (e.g.: Araújo et al. 2005; Elith et al. 2006; Peterson et al. 2007; Costa et al. 2010), porém os resultados variam conforme a situação e a espécie em questão. Elith et al. (2006) testaram diversos modelos em seis regiões do mundo e viram que, em geral, os modelos que utilizam somente dados de presença são menos precisos do que os que utilizam dados de presença e alguma forma de ausência ou pseudo ausência. Dois modelos muito utilizados na literatura, Maxent e GARP, foram testados e avaliados baseado em pontos independentes e fazendo previsões para a mesma área e para áreas diferentes e na primeira situação o Maxent teve melhor performance (Elith et al. 2006) e na segunda o GARP se saiu melhor (Peterson et al. 2007).

Alguns trabalhos defendem a ideia de que a melhor previsão da distribuição das espécies seria a obtida com o uso do consenso de vários modelos (Araújo et al. 2005; 
Araújo \& New 2007). Essa abordagem teria a vantagem de reduzir as incertezas próprias de cada modelo e melhorar a precisão da previsão (Araújo et al. 2005). Tal abordagem já vem sendo usada em várias áreas do conhecimento para melhorar previsões, como na economia, gestão, sistemática, biomedicina, meteorologia e climatologia (Araújo e New, 2007).

\section{APRESENTAÇÃO DA TESE}

O objetivo geral desta tese é analisar os padrões biogeográficos históricos, atuais e futuros dos primatas do Cerrado e as implicações para a conservação do grupo.

Além do capítulo de introdução geral, este documento está organizado em mais três capítulos.. O capítulo 2 analisa as lacunas de conservação e as prioridades atuais para a conservação das espécies. $\mathrm{O}$ capitulo 3 analisa como as populações de primatas reagiram às mudanças climáticas ocorridas antes, durante e depois da última era glacial

e quais seriam as áreas de refúgio climático. O capítulo 4 aborda o efeito que o aquecimento global e as mudanças no uso da terra possivelmente terão para a conservação dos primatas. O capítulo 5 sintetiza as conclusões encontradas na tese e discute limitações e perspectivas para a conservação. 


\section{REFERÊNCIAS BIBLIOGRÁFICAS}

Aguiar, L. M. S., R. B. Machado, and J. Marinho-Filho. 2004. A Diversidade Biológica do Cerrado.in L. M. S. Aguiar and A. Camargo, editors. Ecologia e Caracterização do Cerrado. Embrapa Cerrados, Planaltina-DF.

Araújo, M. B., R. J. Whittaker, R. J. Ladle and M. Erhard. 2005. Reducing uncertainty in projections of extinction risk from climate change. Global Ecology and Biogeography 14: 529-538.

Araújo, M. B. and M. New. 2007. Ensemble forecasting of species distributions. Trends in Ecology and Evolution 22: 42-47.

Bigarella, J. J. and D. Andrade-Lima. 1982. Paleoenvironmental changes in Brazil. Pages 27-40 in G. T. Prance, editor. Biological Diversification in the Tropics. Columbia University Press, New York.

Chapin, F. S., P. A. Matson, and H. A. Mooney. 2002. Principles of Terrestrial Ecosystem Ecology. Springer Science, New York.

Costa, G. C., Nogueira, C., Machado, R. B. and G. R. Colli. 2010. Sampling bias and the use of ecological niche modeling in conservation planning: a field evaluation in a biodiversity hotspot. Biodiversity and Conservation 19: 883-899.

Cracraft, J. 1994. Species Diversity, Biogeography, and the Evolution of Biotas. American Zoologist 34: 33-47.

Decanini, D. P. and Macedo, R. H. 2008. Sociality in Callithrix penicillata: II. Individual Strategies During Intergroup Encounters. International Journal of Primatology 29: 627-639.

Eiten, G. 1972. The Cerrado Vegetation of Brazil. Botanical Review 38:201-341.

Eiten, G. 1994. Vegetação do Cerrado. Pages 17-73 in M. Pinto, editor. Cerrado: caracterização, ocupação e perspectivas. Editora da Universidade de Brasília, Brasília, DF.

Elith, J., C.H. Graham, R. P. Anderson, M. Dudık, S. Ferrier, A. Guisan, R. J. Hijmans, F. Huettmann, J. R. Leathwick, A. Lehmann, J. Li, L. G. Lohmann, B. A. Loiselle, G. Manion, C. Moritz, M. Nakamura, Y. Nakazawa, J. M. Overton, A. T. Peterson, S. J. Phillips, K. Richardson, R. Scachetti-pereira, R. E. Schapire, J. Soberón, S. Williams, M. S. Wisz, N. E. Zimmermann, 2006. Novel methods improve prediction of species ' distributions from occurrence data. Ecography 29: 129-151,

Elton, C. 1962. Animal Ecology. Sidgwick \& Jackson, London UK, 204p.

Fleagle, J. G. 1999. Primate Adaptation and Evolution. 2nd edition. Elsevier Academic Press.

Goldani, A., G. S. Carvalho and J. C. Bicca-Marques. 2006. Distribution patterns of Neotropical primates (Platyrrhini) based on Parsimony Analysis of Endemicity. Brazilian Journal of Biology, 66: 61-74.

Grinnel, J. 1917. The niche relationships of the california thrasher. Auk 34: 427-433.

Haffer, J. 1969. Speciation in Amazonian forest birds. Science 165:131-166.

Hanya, G., P. Stevenson, M. van Noordwijk, S. Te Wong, T. Kanamori, N. Kuze, S. I. Aiba, C. A. Chapman, C. van Schaik, Seasonality in fruit availability affects frugivorous primate biomass and species richness. Ecography 34: 1009-1017.

Hirsch, A., L. G. Dias, L. O. Martins, R. F. Campos, E. C. Landau, N. A. T. Resende. 2002. BDGEOPRIM - Database of geo-referenced localities of neotropical primates. Neotropical Primates, 10: 79-84. 
Hutchinson, G. E. 1958. Concluding Remarks. Cold Spring Harbor Symposia on Quantitative Biology 42: 415-427.

IPCC. 2014. Climate Change 2014: Synthesis report.

Jones, C. B. 1987. Evidence Supporting the Pleistocene Forest Refuge Hypothesis for Primates. Biotropica 19: 373-375.

Kay, R. F., R. H. Madden, C. van Schaik and D. Higdon. 1997. Primate species richness is determined by plant productivity: Implications for conservation. Proceedings of the National Academy of Sciences USA 94: 13023-13027.

Kinzey, W. G. 1982. Distribution of Primates and forest refuges. Pages 455-482 in G. T. Prance, editor. Biological Diversification in the Tropics. Columbia University Press, New York, NY.

Klink, C. and R. B. Machado. 2005. Conservation of the Brazilian Cerrado. Conservation Biology 19:707-713.

Lazaro-Perea, C. 2001. Intergroup interactions in wild common marmosets, Callithrix jacchus: territorial defence and assessment of neighbours. Animal Behaviour. 62:11-21.

Lehman, S. M., J. G. Fleagle, 2006. Primate Biogeography: Progress and Prospects. Springer, New York. 553p.

Machado, R. B., L. M. S. Aguiar, A. A. J. F. Castro, C. C. Nogueira, and M. B. R. Neto. 2008. Caracterização da Fauna e Flora do Cerrado.in F. Faleiro and A. Neto, editors. Savanas - desafios e estratégias para o equilíbrio entre sociedade, agronegócio e recursos naturais. Embrapa Cerrados, Planaltina- DF.

Machado, R. B., M. B. R. Neto, P. G. P. Pereira, E. F. Caldas, D. A. Gonçalves, N. S. Santos, K. Tabor, and M. Steininger. 2004. Estimativas de perda da área do Cerrado brasileiro. Brasília-DF.

Méio, B. B., C. V. Freitas, L. Jatobá, M. E. F. Silva, J. F. Ribeiro and R. P. B. Henriques. 2003 Influência da flora das florestas Amazônica e Atlântica na vegetação do cerrado sensu stricto. Revista Brasileira de Botânica 26: 437-444.

Mittermeier, R. A., Robles-Gil, P., Hoffmann, M., Pilgrim, J. D., Brooks, T. B., Mittermeier, C. G., Lamoreux, J. L. and Fonseca, G. A. B. 2004. Hotspots Revisited: Earth's Biologically Richest and Most Endangered Ecoregions. CEMEX, Mexico City, Mexico 390pp.

Myers, N., R. A. Mittermeier, C. G. Mittermeier, G. A. B. Fonseca, and J. Kent. 2000. Biodiversity hotspots for conservation priorities. Nature 403:853-858.

Oliveira-Filho, A. T. and J. A. Ratter. 1995. A study of the origin of central brazilian forests by the analysis of plant species distribution patterns. Edinburgh Journal of Botany 52:141-194.

Paglia, A. P., G. A. B. Fonseca, A. B. Rylands, G. Herrmann, L. M. S. Aguiar, A. G. Chiarello, Y. L. R. Leite, L. P. Costa, S. Siciliano, M. C. M. Kierulff, S. L. Mendes, V. d. C. Tavares, R. A. Mittermeier, and J. L. Patton. 2012. Lista Anotada dos Mamíferos do Brasil. $2^{\mathrm{a}}$ edition. Conservation International, Arlington, VA.

Peterson, A. P., M. Papes, M. Eaton. 2007. Transferability and model evaluation in ecological niche modeling a comparison of GARP and Maxent. Ecography 30: 550-560.

Peterson, A. T., J. Soberón, R. G. Pearson, R. P. Anderson, E. Martinez-Meyer, M. Nakamura, \& M. B. Araújo. (2011). Ecological Niches and Geographic Distributions. Princeton, USA: Princeton University Press.

Polechová, J. and D. Storch. 2008. Ecological Niche. In: S. Jorgensen and B. Fath. Enciclopedia of Ecology. Elsevier Academic Press, Oxford UK. 
Prance, G. T. 1982. Biological Diversification in the Tropics. Columbia University Press, New York, 1982.

Qiao, H., J. Sóberon and T. A. Peterson. 2015. No Silver Bullets in Correlative Ecological Niche Modeling: Insights from Testing Among Many Potential Algorithms for Niche Estimation. Methods in Ecology and Evolution.

Ratter, J. A., J. F. Ribeiro, and S. Bridgewater. 1997. The Brazilian Cerrado Vegetation and Threats to its Biodiversity. Annals of Botany 80:223-230.

Redford, K. H. and G. A. B. Fonseca. 1986. The role of Gallery Forests in the Zoogeography of the Cerrado' s non-volant mammalian fauna. Biotropica 18:126-135.

Ribeiro, J. F. and Walter, B. M. T. 1998. Fitofisionomias do bioma Cerrado. In: S. Sano and S. Almeida, editors. Cerrado: Ambiente e Flora. Embrapa -CPAC, Planaltina- DF

Rodrigues, M. G. 1992. Sazonalidade da dieta de vertebrados frugivoros em uma dieta semidecidua no Brasil. Dissertação de mestrado. Unicamp, 114p.

Sacramento, T. S. 2014. Influência da disponibilidade de alimentos sobre os comportamentos de um grupo de Sapajus libidinosus e análise das interações e conflitos entre humanos e macacos-prego no Parque Nacional de Brasília, DF. Dissertação de mestrado. Universidade de Brasília.

Salgado-Labouriau, M. L. 1994. História Ecológica da Terra. Editora Edgard Blucher Ltda., São Paulo - SP Brazil.

Schwarzkopf, L. and Rylands, A. B. 1989. Primate Species Richness in Relation to Habitat Structure in Amazonian Rainforest Fragments. Biological Conservation 48:1-12

Silva, J. M. C. and D. C. Oren. 1986. Application of parsimony analysis of endemicity in Amazonian biogeography: an example with primates. Biological Journal of the Linnean Society. 59: 427-437.

Silva, J. M. C. and M. P. D. Santos. 2005. A importância relativa dos processos biogeográficos na formação da avifauna do Cerrado e de outros biomas brasileiros. In: Scariot, A., Souza-Silva, J.C., Felfili, J.M. (Eds.), Cerrado: ecologia, biodiversidade e conservação. Ministério do Meio Ambiente, Brasília, pp. 220-233.

Silva, J. M. C. 1995. Biogeographic analysis of the South American Cerrado avifauna. Steenstrupia 21:49-67.

Silva, J. M. C. 1997. Endemic bird species and conservation in the Cerrado Region, South America. Biodiversity and Conservation, 6: 435-450.

Silva, J. M. C. d. and J. M. Bates. 2002. Biogeographic patterns and conservation in the South American Cerrado: A tropical savanna hotspot. BioScience 52:225-234.

Soberon, J. 2007. Grinnellian and Eltonian niches and geographic distributions of species. Ecology Letters 10: 1115-1123.

Stevenson, P. 2001. The relationship between fruit production and primate abundance in Neotropical communities. Biological Journal of the Linnean Society 72: 161178.

Vilela, S. L. and D. S. Faria. 2002. Dieta do Callithrix penicillata (Primates, Callithrichidae) em áreas de Cerrado no Distrito Federal, Brasil. Neotropical Primates 10: 17-20.

Warner, M. D. 2002. Assessing Habitat Utilization by Neotropical Primates: A New Approach. Primates 43:59-71. 
CAPÍTULO 2

Prioridades para a conservação de primatas do Cerrado e identificação de lacunas de conservação 


\section{Conservation priorities for primates of the Cerrado and identification of conservation gaps}

\section{Danilo Gustavo R. de Oliveira ${ }^{1}$; Fabiano Rodrigues de $\mathrm{Melo}^{2}$ e Ricardo Bomfim Machado 1}

1-Laboratório de planejamento para conservação da biodiversidade; Departamento de Zoologia, Universidade de Brasília.

Instituto de Ciências Biológicas - Campus Darcy Ribeiro

Asa Norte

70910-900 - Brasília, DF - Brasil.

2- Universidade Federal de Goiás, Instituto de Ciências Biológicas, Campus Jataí.

Br 364, km 195, no. 3.800

Parque Industrial

75801615 - Jataí, GO - Brasil.

Corresponding author: Danilo Gustavo R. de Oliveira. E-mail:

danilo.gustavo@gmail.com 


\section{RESUMO}

Primatas são animais associados às formações florestais e isso os torna mais suscetíveis a sofrer os efeitos do desmatamento, tanto que é a ordem de mamíferos com mais espécies ameaçadas de extinção no Brasil. O objetivo do trabalho foi avaliar a eficiência do atual sistema de áreas protegidas do Cerrado quanto à proteção de primatas e apontar áreas que complementem as já existentes. Para tanto, foi realizada uma análise de lacunas de conservação dos primatas a partir dos pontos de ocorrência e de modelagem de nicho ecológico. Foram feitos modelos de nicho com nove algoritmos diferentes e analisado o consenso entre as predições com AUC maior que 0,8. Nos modelos de distribuição, as variáveis mais importantes foram sazonalidade da temperatura e precipitação anual. A priorização foi feita com Marxan, com metas baseadas nos critérios da IUCN. Cruzaram-se os mapas de pontos e modelos com o de unidades de conservação para identificar quais espécies não estão protegidas no Cerrado. Dez táxons já perderam mais de $50 \%$ da sua área esperada e seis perderam mais de $70 \%$. De 15 táxons cinco constituem lacunas de conservação na abordagem por pontos e somente uma na abordagem por modelos. As áreas prioritárias apontadas consistem em algumas áreas grandes e contíguas ao norte e oeste do Cerrado e áreas pequenas e isoladas ao sul, devido à proporção de áreas nativas remanescentes. A conservação do grupo na região pede urgência, pois um terço das espécies não foram observadas em nenhuma unidade de conservação e as áreas apontadas como de maior relevância para conservação do grupo estão na porção mais fragmentada.

Palavras-chave: Priorização para conservação, lacunas de conservação, primatas, modelos de nicho ecológico, consenso de projeções 


\begin{abstract}
Neotropical primates live associated with arboreal formations making them particularly vulnerable to the effects of deforestation, such that they are the order of mammals with the largest number of endangered species in Brazil. The aim of this study was to evaluate the current protected area network in the Cerrado biome regarding the protection of primates and to identify priority areas to complement the existing ones. In order to do that, it was conducted a gap analysis and spatial conservation priority exercise using occurrence records and ecological niche models. The niche models were built with nine different algorithms and the consensus summarized among the ones with AUC greater than 0.8. In the models, the most important variables were seasonal temperature and annual precipitation. The prioritization was made with Marxan with targets based on the IUCN criteria. We crossed the map points and models with the protected areas to identify which species are not protected within the Cerrado. Ten taxa have lost more than $50 \%$ of their expected areas and six have lost more than $70 \%$. Out of the total of 15 taxa, five constitute gaps in the points approach and one in the distribution models approach. Identified priority areas constitute some large areas to the north and west of Cerrado and small and isolated ones to the south, due to the proportion of remnant native areas. The conservation of the group in the region demands urgency because one third of the taxa were not observed in a single protected area and the most relevant priority areas for the conservation of the group are in the most anthropized region.
\end{abstract}

Keywords: Conservation prioritization, conservation gaps, primates, ecological niche models, ensemble forecast 


\section{Introduction}

Primate richness in Brazil is the largest in the world. Brazil has 118 recognized species of primates (Paglia et al. 2012); 34 of them are endangered, making primates the mammal order with the largest number of endangered species (MMA 2014). As they are species which depend on some kind of arboreal stratum (Fleagle 1999), they are associated to the extent of forested area in the location (Reed and Fleagle 1995), which makes them even more vulnerable to the effects of habitat loss.

Fifteen primate taxa have been recorded within Cerrado areas and five of them are on the threatened species list (Hirsch et al. 2002, MMA 2014). According to Hirsch et al. (2002), the Cerrado presents a low quantity of occurrence records for primates. On average $30 \%$ of the primate recordswere obtained from protected areas and in the Cerrado occurrence records have been noted in 8 national parks and in 5 ecological stations and none were found in the other integral protection protected areas.

The Cerrado is the second largest biome of South America, occupying a central position in the continent, and is bordered by several other biomes (IBGE 2004). It is bordered by Chaco and Caatinga in the southwest-northeast diagonal, linking open area vegetations, to the Amazon and the Atlantic Forest in the northwest-southeast diagonal, connecting the vegetation of these two great forest formations (Oliveira-Filho and Ratter 1995). It is a very heterogeneous biome, forming a mosaic of phytophysiognomies that contain grasslands, savannas and forests (Eiten 1972, 1994).

Due to the great concentration of endemic species (mainly trees) and high level of anthropic modifications observed in the Cerrado, the biome was considered a hotspot for biodiversity conservation (Myers et al. 2000). From the total original area of the Cerrado $\left(2,045,064 \mathrm{~km}^{2}\right) 48.5 \%$ had already been devastated by 2010 (MMA/IBAMA $2011 \mathrm{~b}$ ) and the remaining native area encompasses more than 10,000 species of plants 
(Ratter et al. 1997) and 1,325 species of terrestrial vertebrates (Machado et al. 2008b), and 251 of which are mammals (Paglia et al. 2012). There are several threats to the biodiversity of the Cerrado: the advance of mechanized agriculture and pastures, soil degradation, invasion of exotic species, increase of frequency and intensity of forest fires, deforestation of riparian forests and hunting (Klink and Machado 2005, Machado et al. 2008a). Recent modifications in the Brazilian environmental legislation allowed a reduction in protection within rural areas and alongside rivers and this may greatly increase the threat to the fauna of the region (Soares-Filho et al. 2014).

Conservation gap analysis started as an initiative to evaluate the protection level that the existing system of protected areas offered to protect target species (Scott et al. 1993, Jennings 2000). By identifying gaps for the conservation of some groups, a necessity of establishing conservation measures for their protection became clear. To select the locations for the implementation of protected areas, many methods and theories have already been used, such as the selection of areas based on the existence of umbrella or emblematic species; the scenic beauty of the region; the theory of island biogeography, considering each area protected as an island surrounded by a matrix of inadequate habitat; on the richness and endemism as indicatives of importance (Andelman and Fagan 2000, Roberge and Angelstam 2004, Watson et al. 2011). Conservation gap analysis and area prioritization for conservation constitue the systematic planning for conservation, a process aimed at efficiently planning regional conservation strategies, optimizing protection. This process uses four principles: representativeness, which is to protect the existing biological diversity as much as possible; efficiency, which means protecting areas at low cost (financial or other costs regarding the implantation of the area); persistence, which represents the continuation of the species in the future; and flexibility, which means selecting the areas in a way 
minimizes conflicts (Watson et al. 2011). A reserve design made without planning may waste valuable resources for conservation and may not provide complementarity for the protection of non-protected species (Pressey et al. 1993, Andelman and Fagan 2000, Possingham et al. 2000).

Prioritization exercise precision is strongly influenced by the scarcity of points of occurrence, bias in the sample of points and by the existence of errors of omission, generating small areas of prioritization and centered in the known points (Rondinini et al. 2006). A powerful tool, that may assist in the definition of priority areas for species with sampling deficiency, is the ecological niche modeling (Elith and Leathwick 2009a, Meller et al. 2014). With this tool, occurrence points of the species and environmental variables are used to generate a surface of the adequacy of the habitats for the species (Peterson et al. 2011). Environmental variables, which define the niche, may be of two types: the Grinnelian condition type, where there is no interaction with the species or are intrinsic characteristics of the environment; or the Eltonian resource type, where the resource is consumed or referent to the interaction among species (Soberón 2007, Polechová and Storch 2008). Hutchinson proposed the niche as a "hypervolume" formed by the multidimensional set of environmental variables (condition or resource) in which the species can reproduce and survive (Hutchinson 1958). The process of niche modeling usually takes into consideration only condition type variables, because variables of the resource type are difficult to obtain in large scale (Peterson et al. 2011). In this study, the niche concept used is the Grinnelian niche (only condition type variables), according to Hutchinson's multidimensional view (Soberón 2007, Polechová and Storch 2008). In the end of the niche modeling process the models have to be validated with different points than the ones used for calibration to test the accuracy of predictions (Peterson et. al. 2011). Ecological niche modeling may help to extrapolate 
the distribution of species for areas in which they have not been sampled yet, by using a low number of points, which makes it a good tool to use in rare species or species which are difficult to observe (Engler et al. 2004, Guisan et al. 2006, Thorn et al. 2009, Marini et al. 2010a, Marini et al. 2010b).

In this paper, the objective was to identify gaps and define priority areas for the conservation of the primates of the Cerrado. We also tested the hypothesis that the variables related to the vegetation structure would be more important than climatic ones when determining species' distributions.

\section{Methods}

\section{$\underline{\text { Species data }}$}

The taxonomic nomenclature adopted was the one used in (Rylands and Mittermeier 2009) and the genus Sapajus was used opposed to Cebus, as recommended by Alfaro and his collaborators (2012a). Only taxa with at least one occurrence point in the Cerrado area were used here, however all the points of occurrence of the species were used for the modeling process. The following taxa of primates were analyzed as belonging to the Cerrado: Alouatta belzebul, A. caraya, A. guariba, Aotus azarae azarae, A. a. infulatus, Callicebus donacophilus, C. nigrifons, C. personatus, Callithrix geoffroyi, C. jacchus, C. penicillata, Mico melanurus, Sapajus cay, S. libidinosus, S. nigritus and $S$. xanthosternos.

Records of occurrence points of the Cerrado primates were obtained by several means available. Initially, the recordings were researched on the digital database of GBIF (www.gbif.org) and specieslink (splink.cria.org.br) which counts with the occurrences of species in several museums around the world. Data from the Brazilian Primate Center of ICMBIO created to generate the list of threatened primates in Brazil was also collected. In addition to it, an extensive survey on the scientific journals 
available in the CAPES database was carried out and the articles that contained georeferenced recordings were compiled in order to complement the distribution of each species.

The recordings obtained, which had neither an indication of the geographic coordinates nor the location of the collection, were disregarded. Those that only had the indication of the collection location were georeferenced through the use of the tool geoLoc, which is available on the website of the specieslink or the tool GEOLocate, of the Natural History Museum of University of Tulane (http://www.museum.tulane.edu/geolocate/default.html). These tools compare the locality names of the recordings to the collection of localities already known present in several collections to suggest the most probable site of that location. A visual inspection was carried out in the points of occurrence of each species and the points that were distant from the distribution maps existing on the website All the world's primates (http://www.alltheworldsprimates.org/Maps.aspx), were excluded, as a way of excluding mistakes on the georeference or on the identification of the species. Points which were less than $20 \mathrm{~km}$ far from each other, approximately, were filtered in order to remove a spatial bias in the sample (Varela et al. 2014).

$\underline{\text { Ecological niche modelling }}$

A set of climatic and environmental variables, which involve precipitation, temperature, net primary productivity and satellite images which correlate to vegetation aspects was used. The climatic data were obtained from the database Worldclim (Hijmans et al. 2005); www.worldclim.org), where 19 bioclimatic variables are available. From these, we used number 1 (annual mean temperature), number 2 (mean diurnal range), number 4 (temperature seasonality), number 12 (annual precipitation), number 15 (precipitation seasonality), number 17 (precipitation of driest quarter), 
number 18 (precipitation of warmest quarter) and number 19 (precipitation of coldest quarter). Variables were selected by removing those which had Pearson correlation coefficient greater than or equal to $90 \%(\mathrm{r}=0.9)$ with other variables, and selecting those with biological significance.

The vegetation variables were net primary productivity, normalized difference vegetation index (NDVI), product MOD43b3, and an image MOD44b VCF (Vegetation Continuous Fields). The net primary productivity was downloaded on the website Atlas of Biosphere (http://www.sage.wisc.edu/atlas) and it was obtained by the IBIS methodology (integrated biosphere simulator; (Foley et al. 1996, Kucharik et al. 2000)) from a 2006 image. The MOD43b3NDVI and the image MOD44bVCF were produced from the image of the MODIS sensor, in the Terra satellite, of year 2010. VCF estimates the quantity of vegetation in each pixel and NDVI is an index calculated from the satellite images which is highly correlated to a low to medium leaf area index (Turner et al. 1999, Wang et al. 2005), which indicates the quantity of the existing vegetation coverage in a location and it is related to the primary productivity. All the images were obtained or resampled for the resolution of 2.5 arc-minutes (approximately $5 \mathrm{~km})$.

The ecological niche modeling of eachspecies were carried out by using nine algorithms, in a way that it is possible to make a forecast using the modeling set available on the biomod2 package (Thuiller et al. 2009, Thuiller et al. 2014). Consequently, the intention was to seek a consensus of the several models in order to have a reduction of the specific uncertainties of each one (Araújo et al. 2005, Marmion et al. 2009). In order to do that, we used models of the biomod2 package which were used in other analyses (Heikkinen et al. 2006, Grenouillet et al. 2011) in addition to presence-only methods or methods which require background points. 
Were utilized: (1) generalized linear model (GLM), a parametric regression method which can be adapted to different error structures; (2) generalized additive model (GAM), semi-parametric regression in which few assumptions about the function structure are made; (3) multivariate adaptive regression splines (MARS), method similar to GAM, although it produces response curves which are connected straight lines while the GAM curves are smoother; (4) flexible discriminant analysis (FDA), similar to MARS, although it is not a regression and it produces generalized classifications to a linear discriminant analysis; (5) classification tree analysis (CTA) which divides the data several times in homogenous pairs and classifies each one in presence or absence until it grows a "tree" which is then "pruned" to an optimum level of complexity; (6) random forest (RF), is a method similar to CTA, but it generates several trees and selects the consensus among them; (7) artificial neural network (ANN), a method which "learns" the response of the species to the environmental variables transmitting the data through a network of artificial "neurons" that modify themselves in each interaction; (8) Maximum entropy approach (Maxent) (Phillips et al. 2004, Phillips et al. 2006) that aims at the maximum entropy probability distribution (widespread or closer to the uniform) subject to the restrictions imposed by the occurrence recordings and environmental variables in the field of study; and (9) surface range envelope (SRE), the simplest method which consists in designing an envelope that contains the maximum and minimum values of all environmental variables. For a review of the methods and applications consult (Hastie et al. 2009, Peterson et al. 2011). Algorithms from 1 to 7 use presence and pseudo-absence data, Maxent uses presence data and a large sample of the background and SRE uses only presence.

Two sets of pseudo-absence points generated by randomly selecting points considered as absences in a surface range envelope (SRE) were utilized as was done in 
other studies (Le Maitre et al. 2008, Marini et al. 2010a). Models with pseudo-absence are more precise, in general, once they present a greater evaluation value than those which utilize only presence (Elith et al. 2006). The models were generated with $75 \%$ of the points of occurrence of the species separated for practice and $25 \%$ to perform the evaluation tests of the model, using geographically structured cross-validation (Boria et al. 2014). The software evaluates the importance of each environmental variable by means of correlation with a randomized prediction in order to compare results of diferent models.

The most widely used method to evaluate niche models' efficiency (also used in other science fields) is the area under the curve (AUC) of the receiver operatingcharacteristic (ROC) (Hanley and McNeil 1982) plot, that analyzes the absence of false negatives (sensitivity) and the absence of false positives (specificity). The AUC values vary between 0 and 1 , in which 0.5 means that the prediction is not different from the random; values from 0.5 to 0.7 are considered bad predictions; above 0.7 , they are considered useful; above 0.8, good and values above 0.9 are very good (Swets 1988). Only models with AUC greater than 0.80 (with good predictions) have been selected to produce the consensus maps. Although AUC is a criticized measure of evalutation (Lobo et al. 2008) it is still the measure used to make the consensus models in biomod2.

For each species, 32 models were calculated (nine algorithms, 2 sets of pseudoabsence and 2 repetitions) and the average value of each pixel regarding all the selected models was used to generate the probability of occurrence of each species in that location in the consensus model, once this was considered one of the best consensus methods (Marmion et al. 2009). To transform the model results into presence-absence maps, the values of environmental adequacy for each pixel were reclassified following a 
threshold that maximizes the sum of the sensitivity and the specificity (Liu et al. 2005, Jiménez-Valverde and Lobo 2007).

Maps of environmental adequacy for each species were crossed with the map of anthropized areas of the Cerrado generated by the Remote Sensing Center of IBAMA for the year 2010 (MMA/IBAMA 2011b) (Figure 1). Thus, we could quantify the extent to which the expected area of occurrence has already been deforested and which species were affected most by the deforestation.

[[Figure 1]]

\section{$\underline{\text { Conservation Gaps }}$}

Maps of occurrence points (observed occurrences) and maps of environmental adequacy (expected occurrences) of the primates were crossed with a map containing the integral protection protected areas (equivalent to categories I to III of IUCN) in the ArcGIS 10.2 to assess the existence of conservation gaps. The species that did not have recordings of occurrence in none of the protected areas of the Cerrado were considered a "real gap" and species considered a "potential gap" are the ones whose potential distribution does not cross with any of the protected areas. All the species which had the expected occurrence inside the protected areas in at least one pixel were considered potential presence.

\section{$\underline{\text { Conservation Priorities }}$}

Conservation priorities analyses were made for the occurrence point records of the species and also for the potential suitability models generated for each species. The software Marxan 2.43 (Ball et al. 2009) was utilized, which is a tool to assist in the conservation planning calculating the planning units that must be protected, reaching the objectives established with the lowest possible cost. In this study, the planning units were hexagons of 10,000 ha. The cost was the deforestation probability of the Cerrado 
calculated through the comparison of land use images - one of 2002 and the other of 2009 - and projected for the year of 2010 (Salmona 2013), using neural networks and Markov chains in the software IDRISI (Eastman 2009). Goals were calculated taking into consideration the criteria of IUCN for the definition of a vulnerable species (lower category for species considered threatened): 10,000 individuals or $20,000 \mathrm{~km}^{2}$ of extension of the occupation or $2,000 \mathrm{~km}^{2}$ of the occurrence area (IUCN 2001). In order to calculate these goals, the average population density related to each genus was considered (Table 1).

The areas that presented $25 \%$ or less of the remaining native vegetation were excluded from the input files for Marxan in order to exclude heavily deforested areas and areas improper for conservation. Planning units are hexagons of 10,000ha, and sometimes smaller, in case they are boarded by a reserve. Boundary length modifier (LBM) were used the on the value of 0.01 and simulations totalized 1,000 repetitions. 
Table 1. Population density values and conservation goals for the primate genera being studied.

\begin{tabular}{|c|c|c|c|c|}
\hline & $\begin{array}{l}\text { Average } \\
\text { (ind. } / \mathrm{km}^{2} \text { ) }\end{array}$ & Range & $\begin{array}{c}\text { Goals } \\
\left(\text { in } \mathrm{km}^{2}\right) *\end{array}$ & Source \\
\hline Alouatta & 7.5 & $\begin{array}{r}0.2- \\
43\end{array}$ & 1333.3 & $\begin{array}{l}\text { (Chiarello and Melo 2001, Codenotti } \\
\text { et al. 2002, Aguiar et al. 2003, Araújo } \\
\text { et al. 2008) }\end{array}$ \\
\hline Aotus & 32 & $12-64$ & 312.5 & $\begin{array}{l}\text { (Fernandez-Duque et al. 2001, } \\
\text { Fernandez-Duque 2007) }\end{array}$ \\
\hline Callicebus & 8 & $1-15$ & 1250.0 & $\begin{array}{l}\text { (Oliveira et al. 2003, Santana et al. } \\
\text { 2008) }\end{array}$ \\
\hline Callithrix & 17 & $3-38$ & 588.2 & $\begin{array}{l}\text { (Chiarello and Melo 2001, São } \\
\text { Bernardo and Galetti 2004, Santana et } \\
\text { al. 2008) }\end{array}$ \\
\hline Mico & 17 & $7-27$ & 588.2 & (Stallings 1985) \\
\hline Sapajus & 22 & $6-66$ & 454.5 & $\begin{array}{l}\text { (Bitetti 2001, Chiarello and Melo } \\
\text { 2001, São Bernardo and Galetti 2004, } \\
\text { Araújo et al. 2008) }\end{array}$ \\
\hline
\end{tabular}

\footnotetext{
*Goal necessary to reach a population of 10,000 adult individuals. In the approach by points, it was considered that each point represented $100 \mathrm{~km}^{2}$ (size of the planning unit) and calculated the quantity of points that were necessary to reach the goal. When the number of points was greater than $50 \%$ of the total of points of the species, $50 \%+1$ points were considered.
}

\section{Results}

$\underline{\text { Niche models }}$

In total, 2,395 occurence points were obtained for all primates associated to the Cerrado. After removing the points with uncertaintities and filter the ones which were 
too close from each other, 1,869 occurrence points remained. Callicebus donacophilus was removed from analysis because it had a low number of test points and the models did not have high evaluation scores. The consensus of the modeling algorithms presented AUC values between 0.82 and 0.99 , with an average of 0.93 .

Binary models generated to each one of the 15 taxa are represented in figure 2, which shows that only three species are mostly distributed in the Cerrado (Alouatta caraya, Callithrix penicillata and Sapajus libidinosus). Seven taxa are present in the Cerrado and the Atlantic Forest (Alouatta caraya, Alouatta guariba, Callicebus nigrifrons, C. personatus, Callithrix geoffroyi, Sapajus nigritus and S. xanthosternos), three are in the border between the Cerrado, Pantanal and Chaco (Aotus azarae azarae, Mico melanurus and Sapajus cay), two are in the border between the Cerrado and the Amazon (Alouatta belzebul and Aotus azarae infulatus) and Callithrix jacchus and Sapajus libidinosus occupies the Cerrado and the Caatinga. By adding all the maps is possible to notice that the richest areas expected are located mainly in the south of the domain, in the states of Sao Paulo, Minas gerais and Mato Grosso do Sul, besides an area located in the center-west of the state of Mato Grosso (figure 3).

As opposed to expected, the variables related to the vegetation were not the most important ones to foresee the occurrence of the species, the most important ones, in average, were temperature seasonality (bio 4) and annual precipitation (bio 12) (table 2). [[Figure 2]]

[[Figure 3]] 
Table 2. Average value of the importance of each variable for environmental suitability forecast generated by the models. Higher value represents higher variable importance. Numbers in columns represent each one of the taxa used: 1- Alouatta belzebul; 2- Alouatta caraya; 3 Alouatta guariba; 4- Aotus azarae azarae; 5-Aotus azarae infulatus; 6-Callicebus nigrifrons; 7 Callicebus personatus; 8-Callithrix geoffroyi; 9- Callithrix jacchus; 10- Callithrix penicillata; 11- Mico melanurus; 12- Sapajus cay; 13-Sapajus libidinosus; 14- Sapajus nigritus; 15-

Sapajus xanthosternos. Bio1 = annual mean temperature, bio2 $=$ mean diurnal range, bio4 $=$ temperature seasonality, bio12 $=$ annual precipitation, bio $15=$ precipitation seasonality, bio $17=$ precipitation of driest quarter, bio18 $=$ precipitation of warmest quarter, bio19 $=$ precipitation of coldest quarter, $\mathrm{VCF}=$ vegetation continuous fields, $\mathrm{NPP}=$ net primary productivity and NDVI $=$ normalized difference vegetation index.

\section{Species}

\begin{tabular}{llllllllllllllll}
\hline & $\mathbf{1}$ & $\mathbf{2}$ & $\mathbf{3}$ & $\mathbf{4}$ & $\mathbf{5}$ & $\mathbf{6}$ & $\mathbf{7}$ & $\mathbf{8}$ & $\mathbf{9}$ & $\mathbf{1 0}$ & $\mathbf{1 1}$ & $\mathbf{1 2}$ & $\mathbf{1 3}$ & $\mathbf{1 4}$ & $\mathbf{1 5}$ \\
\hline Bio1 & 0.06 & 0.13 & 0.10 & 0.24 & 0.16 & 0.15 & 0.13 & 0.14 & 0.08 & 0.18 & 0.27 & 0.36 & 0.09 & 0.13 & 0.12 \\
Bio2 & 0.05 & 0.07 & 0.18 & 0.07 & 0.07 & 0.12 & 0.08 & 0.06 & 0.15 & 0.05 & 0.12 & 0.10 & 0.15 & 0.04 & 0.38 \\
Bio4 & 0.26 & 0.48 & 0.28 & 0.39 & 0.26 & 0.40 & 0.57 & 0.62 & 0.39 & 0.38 & 0.41 & 0.32 & 0.13 & 0.58 & 0.42 \\
Bio12 & 0.07 & 0.35 & 0.15 & 0.20 & 0.25 & 0.22 & 0.32 & 0.23 & 0.29 & 0.28 & 0.13 & 0.38 & 0.42 & 0.14 & 0.21 \\
Bio15 & 0.15 & 0.10 & 0.22 & 0.17 & 0.11 & 0.19 & 0.08 & 0.10 & 0.06 & 0.06 & 0.12 & 0.14 & 0.24 & 0.05 & 0.05 \\
Bio17 & 0.14 & 0.10 & 0.24 & 0.45 & 0.12 & 0.10 & 0.18 & 0.17 & 0.09 & 0.15 & 0.29 & 0.22 & 0.35 & 0.12 & 0.28 \\
Bio18 & 0.20 & 0.05 & 0.34 & 0.13 & 0.65 & 0.33 & 0.23 & 0.14 & 0.10 & 0.07 & 0.15 & 0.30 & 0.07 & 0.13 & 0.11 \\
Bio19 & 0.29 & 0.26 & 0.31 & 0.31 & 0.11 & 0.22 & 0.19 & 0.16 & 0.15 & 0.25 & 0.35 & 0.32 & 0.13 & 0.09 & 0.07 \\
VCF & 0.03 & 0.05 & 0.05 & 0.15 & 0.10 & 0.04 & 0.03 & 0.06 & 0.07 & 0.05 & 0.03 & 0.16 & 0.18 & 0.02 & 0.07 \\
NPP & 0.05 & 0.15 & 0.10 & 0.13 & 0.11 & 0.05 & 0.01 & 0.02 & 0.12 & 0.14 & 0.04 & 0.16 & 0.26 & 0.03 & 0.02 \\
NDVI & 0.10 & 0.04 & 0.10 & 0.14 & 0.11 & 0.09 & 0.17 & 0.20 & 0.21 & 0.04 & 0.09 & 0.27 & 0.21 & 0.23 & 0.05 \\
\hline
\end{tabular}

Great part of the primate richness in the Cerrado is located in the south of the biome (figure 3), which is exactly where most part of the deforestation of the region happened (Figure 1). Two thirds of the taxa have already lost more than $50 \%$ of its expected area, and six of them have already lost more than 70\% (Table 3) because of land use changes. 
Table 3. Quantity of the expected area of occurrence (in $\mathrm{km}^{2}$ ) of taxa of primates in the Cerrado that has been deforested until 2010.

\begin{tabular}{lrrrr}
\hline \multicolumn{1}{c}{ Species } & $\begin{array}{c}\text { Area in the } \\
\text { Cerrado }\end{array}$ & $\begin{array}{c}\text { Anthropized } \\
\text { area }\end{array}$ & $\begin{array}{c}\text { Anthropized } \\
\text { percentage }\end{array}$ & $\begin{array}{c}\text { Remaining } \\
\text { area }\end{array}$ \\
\hline Alouatta belzebul & 75959.6 & 17269.8 & 22.7 & 58689.8 \\
Alouatta caraya & 1479850.1 & 835624.9 & 56.5 & 644225.2 \\
Alouatta guariba & 212155.9 & 151965.4 & 71.6 & 60190.5 \\
Aotus azarae azarae & 207723.0 & 150372.3 & 72.4 & 57350.7 \\
Aotus azarae & & & & \\
infulatus & 402470.5 & 110637.8 & 27.5 & 291832.6 \\
Callicebus nigrifrons & 224092.4 & 161939.4 & 72.3 & 62153.0 \\
Callicebus & & & & \\
personatus & 161847.1 & 109183.3 & 67.5 & 52663.8 \\
Callithrix geoffroyi & 68363.6 & 31676.8 & 46.3 & 36686.9 \\
Callithrix jacchus & 198995.7 & 52109.7 & 26.2 & 146886.0 \\
Callithrix penicillata & 1216508.0 & 740063.6 & 60.8 & 476444.5 \\
Mico melanurus & 312704.2 & 215088.0 & 68.8 & 97616.2 \\
Sapajus cay & 338193.4 & 244317.5 & 72.2 & 93875.9 \\
Sapajus libidinosus & 1016635.0 & 440381.0 & 43.3 & 576254.0 \\
Sapajus nigritus & 105189.0 & 80992.8 & 77.0 & 24196.3 \\
Sapajus & & & & \\
xanthosternos & 23.1 & 23.1 & 100.0 & 0.0 \\
\hline
\end{tabular}

\section{Conservation priorities}

In the prioritization by models approach, the conservation goals are possible to being reached for all the taxa in case the planning units pointed out are effectively protected. Nonetheless, in the prioritization by points it was not possible to reach the goal established in seven taxa, which means that the quantity of points of occurrence known that still have native vegetation is not sufficient to reach the goals established.

The best solutions to the prioritization by points and by models are represented in figure 4. Prioritization by using maps of niche models provided a more compact solution selecting an area of 3,864,084.1 ha against 7,041,219.9 ha of the solution with occurrence points - the area of the existing protected areas were already considered. 
Cost of the solution by the models approach was $9.6 \%$ lower than the cost of the solution by points. By adding all the times that a certain cell was selected for a prioritization solution, we have its irreplaceability index, which indicates the importance of that planning unit (Figure 5). Many small areas were selected in common in the two approaches and have a high value of irreplaceability, especially to the south of the domain. The largest and more continuous areas of prioritization were pointed out to the north and the west of the biome, due to the greater quantity of remaining native area. Only one great and continuous area was pointed in the southeast (Figure 4).

[[Figure 4]]

[[Figure5]]

\section{Conservation Gaps}

Five of the 15 taxa analyzed do not present points of occurrence in any of the protected areas (PA) in the Cerrado, constituting real conservation gaps. They are the following: Sapajus xanthosternos, Aotus azarae azarae, Callicebus nigrifrons, Alouatta belzebul and A. guariba. In four of the other ten taxa (Callicebus personatus, Callithrix geoffroyi, Sapajus cay and S. nigritus) the area inside a PA was less than the conservation goal (table 4). 
Table 4. Efficiency of the current reserve network of the Cerrado for protection of primates.

\begin{tabular}{|c|c|c|c|}
\hline Species & $\begin{array}{c}\text { Number of } \\
\text { occurrences in } \\
\text { the } \\
\text { Planning units } \\
\end{array}$ & $\begin{array}{l}\text { Percentage of the } \\
\text { goals in the } \\
\text { existing reserves } \\
\text { (points) }\end{array}$ & $\begin{array}{l}\left.\text { Area (in } \mathrm{km}^{2}\right) \text { in } \\
\text { the existing } \\
\text { reserves (niche } \\
\text { models) }\end{array}$ \\
\hline$\overline{\text { Alouatta belzebul }}$ & 12 & $0 \%$ & 3176.8 \\
\hline Alouatta caraya & 124 & $100 \%$ & 37287.4 \\
\hline Alouatta guariba & 12 & $0 \%$ & 5255.7 \\
\hline Aotus azarae azarae & 0 & $0 \%$ & 2730.9 \\
\hline Aotus azarae infulatus & 22 & $100 \%$ & 11782.8 \\
\hline Callicebus nigrifrons & 20 & $0 \%$ & 4531.7 \\
\hline Callicebus personatus & 20 & $7.2 \%$ & 4816.4 \\
\hline Callithrix geoffroyi & 22 & $53.8 \%$ & 3563.5 \\
\hline Callithrix jacchus & 8 & $100 \%$ & 8527.4 \\
\hline Callithrix penicillata & 144 & $100 \%$ & 19229.2 \\
\hline Mico melanurus & 22 & $100 \%$ & 2511.2 \\
\hline Sapajus cay & 18 & $63 \%$ & 3315.6 \\
\hline Sapajus libidinosus & 52 & $100 \%$ & 37305.9 \\
\hline Sapajus nigritus & 18 & $0.6 \%$ & 3059.0 \\
\hline Sapajus xanthosternos & 2 & $0 \%$ & 0.0 \\
\hline
\end{tabular}

Regarding the area of environmental suitability of each species, only Sapajus xanthosternos did not present potential presence in any of the protected areas of the Cerrado and it constitutes a potential gap. On average, the taxa are potentially present in $37.3 \%( \pm 34.3 \%)$ of the protected areas, with Callithrix penicillata as the most widely distributed occupying $81.7 \%$ of the reserves.

\section{Discussion}

$\underline{\text { Predictor variables of the presence of primates }}$

Environmental variables that influence the occurrence of primates the most in the Cerrado are temperature seasonality and annual precipitation. The precipitation is traditionally seen as important for primate richness (Reed and Fleagle 1995, Kay and Madden 1997, Kamilar 2009). Nevertheless, the primary productivity was suggested as the best factor that determines the richness of species of primates in a region because 
the two variables increase together up to a precipitation of 2,500 $\mathrm{mm} / \mathrm{year}$ and then decrease in rainier areas (Kay et al. 1997). Considering that the productivity of plants is proportional to the net primary productivity (NPP), the outcome of our study does not show NPP as an important variable when foreseeing the occurrence of primates, as opposed to what Kay et al. (1997) suggested. Probably, this has occurred because the richness of frugivore primates is associated to the productivity and the fruit seasonality (Hanya et al. 2011) and the Cerrado has low diversity of frugivore animals.

In studies with primates of the Atlantic Forest, the variables indicated as determinant to the distribution of species were: minimum temperature, maximum temperature, average temperature, precipitation, relative humidity and rainy days (Vilanova et al. 2005, Grelle and Cerqueira 2006). Boubli and Lima (2009) tested the requirements of niche for Cacajao spp. and Chiropotes israelita and realized that besides having association with some ecoregions of the Amazon, the animals were also associated to isothermality, which is highly correlated to temperature seasonality $(\mathrm{r}=-$ $0,90)$.

Temperature seasonality is the most important variable for the occurrence of primates in the Cerrado. Some studies have suggested the existence of an association between precipitation seasonality (Kay and Madden 1997, Kamilar 2009), seasonality of fruit productivity (Hanya et al. 2011) or isothermality (Boubli and Lima 2009) and quantity of species of primates, but not between temperature seasonality. This may have occurred because the Cerrado has a very remarkable seasonality between the rainy season and the drought and the dry environment has a wider range of variation of the daily temperature than an environment with a greater concentrarion of water vapor (Geerts 2003), generating higher seasonality. Hence, the animals which inhabit the 
region would have adapted better to tolerate the temperature seasonality found in Cerrado than any other environmental characteristic.

\section{$\underline{\text { Threat Status }}$}

Despite only five species of primates being in the list of threatened animals in Brazil (MMA 2014), analysis of the distribution of animals with the map of deforestation in the Cerrado domain points out some concerns. Ten out of the 15 taxa analyzed already had more than $50 \%$ of their potential area of occurrence deforested. If we take into consideration the average annual deforestation rate between 2002 and 2010 of 1,240,000 hectares per year (MMA/IBAMA 2009, 2011a, b) as a basis, we conclude that six taxa could have lost $50 \%$ of the Cerrado area in the last ten years (table 3 ), which would imply in a regional extinction threat level for the Cerrado (IUCN 2003). Out of these six taxa Aotus azarae azarae, Callicebus nigrifrons and Sapajus nigritus are not considered under extinction threat in a national level, which suggests that a greater quantity of species could be regionally threatened (IUCN 2003). These concerns are enhanced because the most deforested area in the Cerrado is also the area with greatest quantity of expected species (Figure 3).

\section{$\underline{\text { Models x Points }}$}

Comparing the conservation prioritization by points and by niche models, the final outcomes of the two processes were similar. However, the prioritization by points remained around the original occurrence points (it only occupied a larger area due to the BLM parameter value), which may maintain an existing collection bias and generate an increase in the conservation only around the areas with more samples (Rondinini et al. 2006). Furthermore, studies for conservation planning with incomplete data demonstrate to be less efficient (Freitag and Van Jaarsveld 1998) and to have reduced flexibility in the areas available to be selected (Gladstone and Davis 2003). Occurrence points use to 
present a greater error of omission, but they can also indicate species in locations in which they do not occur (commission) due to georeference or taxonomic identification errors, while the models approach use to present greater error of comission and levels of uncertainty (Rondinini et al. 2006). For this reason, a combined analysis of the two methodologies decrease the disadvantages associated to each one of them.

In the two prioritization approaches it was preferable to increase areas around the existing protected areas and in more continuous areas in the north of the Cerrado, where there is a greater quantity of remaining areas, and more isolated areas in the south, where there was a strong habitat fragmentation (Figure 1 and 4). This result occurred due to high level of anthropization of the areas in the south of the biome and low cost of inclusion of planning units in the north of the Cerrado because they have smaller probability of deforestation (Salmona 2013). Another reason why outcome does not directly reflect richness is that Marxan's "greedy" method gives preferences to the selection of low cost areas, firstly. The inclusion of only one isolated unit smaller than 11,570 ha, as proposed in points of the south of the domain, is not enough to maintain a genetically stable population of a large bodied primate, the muriqui (Brito and Grelle 2006). It is necessary to maintain a larger and connected area so the gene flow and the population do not suffer the effects of inbreeding and end up being extinct (Saccheri et al. 1998, Coulon et al. 2004, Fagan and Holmes 2006).

Main differences between the two approaches lie on the fact that the points of occurrence did not seem sufficient to reach a conservation goal determined in almost half the taxa and generated a solution with a greater quantity of planning units and with a higher cost. The non-accomplishment of the goals may possibly be attributed to the fact that the occurrence points have a bias of sampling and a low density of recordings in the Cerrado, besides wide anthropized area which has reduced the availability of 
habitats for the species (Figure 1). Analysis by models provided a more compact and lower cost solution and reached all conservation goals established, which indicates a more efficient solution and easier to be implemented. Ecological niche models have proven themselves to be good tools to assist analyses of conservation prioritization (Elith and Leathwick 2009a), especially in studies considering the effect of climate changes in the future (Araujo 2009, Faleiro et al. 2013).

\section{Conservation prioritization}

Five taxa do not present occurrence records in any protected areas of the Cerrado, and four of them present potential occurrence inside the units. All the potential and real gaps in protected areas of the Cerrado also occur in regions outside the Cerrado and have been recorded in protected areas of Amazonia and Atlantic Forest. The difference, apparently contradictory, between the real and the potential conservation gaps may be explained by one of two possibilities. First, the niche models may have foreseen the occurrence of the species in a location that it does not occupy in practice because it does not have access by movement or it does have access and is competitively excluded by other species that inhabit the location (Soberón and Peterson 2005, Peterson et al. 2011), such as Alouatta guariba and A. caraya or Callicebus personatus and Callithrix geoffroyi, which may occur in sympatry. Second, the species may exist in the location and have not been sampled, which implies in the necessity of more field studies to ascertain the presence of the species in the established reserves.

In this study, a total of $66.7 \%$ of the taxa are represented by occurrence points in protected areas of the Cerrado. In similar situations, Pinto and Grelle (2009) found out that the existing protected area network protects $89 \%$ of the species in the Atlantic Forest. In the Amazon, though, an analysis has been made considering multiple scales and situations and in the closest to our methodology, with rectangular units of 
$0.25^{\circ} \times 0.25^{\circ}$ and considering only integral protection protected areas, the current network protects $59.8 \%$ of the species of primates (Pinto et al. 2014). The protection of primates is not greater than the random expected in the Amazon (Pinto et al. 2014) or in the Atlantic Forest, when we consider only reserves larger than 11,570 ha (Pinto and Grelle 2009), which indicates that the Brazilian reserves, in general, were not projected for conservation of this group.

Areas in the Serra do Espinhaço and in the Peruaçu Valley in Minas Gerais, the Serra da Bodoquena in Mato Grosso do Sul, the region between the Mortes river and the Araguaia River in Mato Grosso and the region south of Maranhão, borded by Piauí, it is already possible to find areas indicated for creation of PAs in the evaluation of the priority areas of the Cerrado (MMA 2002, 2006). Other studies have evaluated the conservation priorities for sites in the Cerrado based on trees (Siqueira and Peterson 2003), endemic vertebrates (Diniz-Filho et al. 2008) and non-flying mammals (Faleiro et al. 2013) with similar results for vertebrates and a different pattern for trees. These creations or expansions of units are important to approximate, with systematic criterion, to Aichi's goal of protecting, until 2020, 17\% of each Brazilian terrestrial area (UICN et al. 2011), because currently the coverage on integral protection areas is present in only $3.12 \%$ of the Cerrado and $9.55 \%$ considering all PAs. Taking into consideration the priority and primate richness in the areas, the Serra do Espinhaço is the preferable area to invest in protected areas.

In the solutions of conservation prioritization calculated in this study, four largest areas are suggested: one in the states of Tocantins, Piauí and Maranhão, other in the states of Tocantis and Mato Grosso, another in the northwest of Mato Grosso and in Minas Gerais. Out of the indicated areas, the formation of a unit mosaic in Tocantins and Maranhão and the creation of an area in the northwest of Mato Grosso seem to be 
the most viable to put into practice. Nonetheless, the most difficult one to concretize is the one in Minas Gerais, which is also the one with the greatest primate richness and highest risk of deforestation. As the recent changes in the use of the land and the probability of deforestation were included as a cost in the prioritization process, the software selected areas where the conflict between human activities and the creation of areas for conservation will be smaller, facilitating the implementation in practice.

\section{$\underline{\text { Conclusions }}$}

Our study found that the most important variables for occurrence of primates in the Cerrado are temperature seasonality and annual mean precipitation. We also found that one third of the taxa of primates are a real conservation gap and one species is a potential conservation gap. The reserve system network must take into consideration these species to complement the total of species already protected.

Finally, we highlight that the Cerrado is a domain which has been deforested at a very high rate and may be extinct soon (Machado et al. 2004); that is the reason why the conservation of the species which inhabit it is so important. Nevertheless, it is still possible to invest in the territories pointed out in figure $4 \mathrm{~b}$, mainly the two big areas in the north of the domain, the one in the west and the other in the southeast, and try to prevent the exclusion of species from the region. For this to happen, governmental incentives are necessary to provide more protection to the areas and more scientific efforts to comprehend areas that have few samples and understand the effects of the changes in the use of the land.

\section{Acknowledgements}


We thank Giovanna Botura and Yuri Salmona for the help regarding the use of the prioritization algorithm. This work was supported by the Ecology program of the University of Brasília. RBM received a research grant from $\mathrm{CNPq}$.

\section{References}

Aguiar, L. M., Reis, N. R., Ludwig, G., \& Rocha, V. J. (2003). Dieta, área de vida, vocalizações e estimativas populacionais de Alouatta guariba em um remanescente florestal no norte do estado do Paraná. Neotropical Primates, $11(78-86$.

Alfaro, J. L. W., Silva, J. D. J., \& Rylands, A. B. (2012). How different are robust and gracile capuchin monkeys? An argument for the use of Sapajus and Cebus. American Journal of Primatology, 74(4), 273-286.

Andelman, S. J., \& Fagan, W. F. (2000). Umbrellas and flagships: efficient conservation surrogates or expensive mistakes? Proceedings of the National Academy of Sciences of the United States of America, 97(11), 5954-5959.

Araujo, M. B. (2009). Climate Change and Spatial Conservation Planning. In A. Moilanen, K. A. Wilson \& H. P. Possingham (Eds.), Spatial conservation prioritization : quantitative methods and computational tools (pp. 328). Oxford, UK: Oxford Universtiy Press.

Araújo, M. B., Whittaker, R. J., Ladle, R. J., \& Erhard, M. (2005). Reducing uncertainty in projections of extinction risk from climate change. Global Ecology and Biogeography, 14(6), 529-538.

Araújo, R. M. D., Souza, M. B. D., \& Ruiz-miranda, C. R. (2008). Densidade e tamanho populacional de mamíferos cinegéticos em duas Unidades de Conservação do Estado do Rio de Janeiro, Brasil. Iheringia, Série Zoológica, 98(3), 391-396.

Ball, I. R., Possingham, H. P., \& Watts, M. (2009). Marxan and relatives: Software for spatial conservation prioritization. In A. Moilanen, K. A. Wilson \& H. P. Possingham (Eds.), Spatial conservation prioritization: Quantitative methods and computational tools (pp. 185-195). Oxford, UK: Oxford University Press.

Bitetti, M. S. (2001). Home-range use by the tufted capuchin monkey (Cebus apella nigritus) in a subtropical rainforesr of Argentina. Journal of Zoology (London), 253(33-45.

Boria, R. A., Olson, L. E., Goodman, S. M., \& Anderson, R. P. (2014). Spatial filtering to reduce sampling bias can improve the performance of ecological niche models. Ecological Modelling, 275(73-77.

Boubli, J. P., \& Lima, M. G. (2009). Modeling the geographical distribution and fundamental niches of Cacajao spp. and Chiropotes israelita in northwestern Amazonia via a Maximum Entropy algorithm. International Journal of Primatology, 30(2), 217-228.

Brito, D., \& Grelle, C. E. V. (2006). Estimating Minimum Area of Suitable Habitat and Viable Population Size for the Northern Muriqui (Brachyteles hypoxanthus). Biodiversity and Conservation, 15(13), 4197-4210.

Chiarello, A. G., \& Melo, F. R. (2001). Primate population densities in Atlantic Forest remnants of northern Espirito Santo, Brazil. International Journal of Primatology, 22(3), 379-396. 
Codenotti, T. L., Silva, V. M., Albuquerque, V. J., Camargo, E. V., \& Silveira, R. M. M. (2002). Distribuição e situação atual de conservação de Alouatta caraya (Humboldt, 1812) no Rio Grande do Sul, Brasil. Neotropical Primates, 10(3), 132-141.

Coulon, A., Cosson, J. F., Angibault, J. M., Cargnelutti, B., Galan, M., Morellet, N., Petit, E., Aulagnier, S., \& Hewison, A. J. (2004). Landscape connectivity influences gene flow in a roe deer population inhabiting a fragmented landscape: an individual-based approach. Molecular ecology, 13(9), 2841-2850.

Diniz-Filho, J. A. F., Bini, L. M., Pinto, M. P., Terribile, L. C., de Oliveira, G., Vieira, C. M., Blamires, D., de Souza Barreto, B., Carvalho, P., Rangel, T. F. L. V. B., Tôrres, N. M., \& Bastos, R. P. (2008). Conservation planning: a macroecological approach using the endemic terrestrial vertebrates of the Brazilian Cerrado. Oryx, 42(04), 567.

Eastman, J. R. (2009). Guide to GIS and Image Processing. Worcester, Massachusetts: Clark University.

Eiten, G. (1972). The Cerrado Vegetation of Brazil. Botanical Review, 38(2), 201-341.

Eiten, G. (1994). Vegetação do Cerrado. In M. Pinto (Ed.), Cerrado: caracterização, ocupação e perspectivas ( $2^{\circ} \mathrm{ed} ., \mathrm{pp} .17-73$ ). Brasília, DF: Editora da Universidade de Brasília.

Elith, J., Graham, C. H., Anderson, R. P., Dudık, M., Ferrier, S., Guisan, A., Hijmans, R. J., Huettmann, F., Leathwick, J. R., Lehmann, A., Li, J., Lohmann, L. G., Loiselle, B. A., Manion, G., Moritz, C., Nakamura, M., Nakazawa, Y., Overton, J. M., Peterson, A. T., Phillips, S. J., Richardson, K., Scachetti-pereira, R., Schapire, R. E., Soberón, J., Williams, S., Wisz, M. S., \& Zimmermann, N. E. (2006). Novel methods improve prediction of species' distributions from occurrence data. Ecography, 29(129-151.

Elith, J., \& Leathwick, J. (2009). The Contribution of Species Distribution Modelling to Conservation Prioritization. In A. Moilanen, K. A. Wilson \& H. P. Possingham (Eds.), Spatial Conservation Prioritization (pp. 70-93). Oxford: Oxford University Press.

Engler, R., Guisan, A., \& Rechsteiner, L. (2004). An improved approach for predicting the distribution of rare and endangered species from occurrence and pseudoabsence data. Journal of Applied Ecology, 41(2), 263-274.

Fagan, W. F., \& Holmes, E. E. (2006). Quantifying the extinction vortex. Ecology Letters, 9(1), 51-60.

Faleiro, F. V., Machado, R. B., \& Loyola, R. D. (2013). Defining spatial conservation priorities in the face of land-use and climate change. Biological Conservation, $158(248-257$.

Fernandez-Duque, E. (2007). Aotinae: Social monogamy in the only nocturnal haplorhines. In C. J. Campbell, A. Fuentes, K. C. Mackinnon, M. Panger \& S. K. Bearder (Eds.), Primates in Perspective (pp. 139-154). Oxford, UK: Oxford University Press.

Fernandez-Duque, E., Rotundo, M., \& Sloan, C. (2001). Density and Population Structure of Owl Monkeys (Aotus azarai ) in the Argentinean Chaco. American Journal of Primatology, 53(99-108.

Fleagle, J. G. (1999). Primate Adaptation and Evolution (2nd ed.): Elsevier Academic Press.

Foley, J. A., Prentice, I. C., Ramankutty, N., Levis, S., Pollard, D., Sitch, S., \& Haxeltine, A. (1996). An integrated biosphere model of land surface processes, 
terrestrial carbon balance, and vegetation dynamics. Global Biogeochemical Cycles, 10(4), 603-628.

Freitag, S., \& Van Jaarsveld, A. S. (1998). Sensitivity of selection procedures for priority conservation areas to survey extent, survey intensity and taxonomic knowledge. Proceedings of the Royal Society B: Biological Sciences, 265(14751482.

Geerts, B. (2003). Empirical estimation of the monthly-mean daily temperature range. Theoretical and Applied Climatology, 74(3-4), 145-165.

Gladstone, W., \& Davis, J. (2003). Reduced survey intensity and its consequences for marine reserve selection. Biodiversity and Conservation, 12(1525-1536.

Grelle, C. E. V., \& Cerqueira, R. (2006). Determinantes da distribuição geográfica de Callithrix flaviceps (Thomas) (Primates, Callitrichidae). Revista Brasileira de Zoologia, 2006(2), 414-420.

Grenouillet, G., Buisson, L., Casajus, N., \& Lek, S. (2011). Ensemble modelling of species distributions: the effects of geographical and environmental ranges. Ecography, 34(1), 9-17.

Guisan, A., Broennimann, O., Engler, R., Vust, M., Yoccoz, N. G., Lehmann, A., \& Zimmermann, N. E. (2006). Using Niche-Based Models to Improve the Sampling of Rare Species. Conservation Biology, 20(2), 501-511.

Hanley, J. A., \& McNeil, B. J. (1982). The meaning and use of the area under a Receiver Operating Characteristic (ROC) curve. Radiology, 143(1), 29-36.

Hanya, G., Stevenson, P., van Noordwijk, M., Te Wong, S., Kanamori, T., Kuze, N., Aiba, S.-i., Chapman, C. A., \& van Schaik, C. (2011). Seasonality in fruit availability affects frugivorous primate biomass and species richness. Ecography, 34(6), 1009-1017.

Hastie, T., Tibshirani, R., \& Friedman, J. (2009). Elements of Statistical Learning Data Mining, Inference and Prediction (2nd ed.). California, US: Springer.

Heikkinen, R. K., Luoto, M., Araújo, M. B., Virkkala, R., Thuiller, W., \& Sykes, M. T. (2006). Methods and uncertainties in bioclimatic envelope modelling under climate change. Progress in Physical Geography, 30(6), 751-777.

Hijmans, R. J., Cameron, S. E., Parra, J. L., Jones, P. G., \& Jarvis, A. (2005). Very high resolution interpolated climate surfaces for global land areas. International Journal of Climatology, 25(15), 1965-1978.

Hirsch, A., Dias, L. G., Martins, L. d. O., Campos, R. F., Landau, E. C., \& Resende, N. A. T. (2002). BDGEOPRIM - Database of geo-referenced localities of Neotropical Primates. Neotropical Primates, 10(79), 79-84.

Hutchinson, G. E. (1958). Concluding remarks. In Cold Spring Harbor Symposia on Quantitative Biology (Vol. 42, pp. 415-427).

IBGE. (2004). Mapa de Biomas do Brasil. Escala 1:5.000.000. In.

IUCN. (2001). IUCN Red List Categories and Criteria Version 3.1. In (pp. 30).

IUCN. (2003). Guidelines for Application of IUCN Red List Criteria at Regional Levels Version 3.0. In I. S. S. Comission (Ed.), (pp. 26). Gland, Switzerland and Cambridge, UK: IUCN.

Jennings, M. D. (2000). Gap analysis concepts, methods, and recent results. Landscape Ecology, 15(5-20.

Jiménez-Valverde, A., \& Lobo, J. M. (2007). Threshold criteria for conversion of probability of species presence to either-or presence-absence. Acta Oecologica, 31(3), 361-369. 
Kamilar, J. M. (2009). Environmental and geographic correlates of the taxonomic structure of primate communities. American Journal of Physical Anthropology, 139(3), 382-393.

Kay, R. F., \& Madden, R. H. (1997). Mammals and rainfall: paleoecology of middle miocene at La Venta (Colombia, South America). Journal of Human Evolution, 32(161-199.

Kay, R. F., Madden, R. H., Van Schaik, C., \& Higdon, D. (1997). Primate species richness is determined by plant productivity: Implications for conservation. Proceedings of the National Academy of Sciences, 94(13023-13027.

Klink, C., \& Machado, R. B. (2005). Conservation of the Brazilian Cerrado. Conservation Biology, 19(3), 707-713.

Kucharik, C. J., Foley, J. A., Delire, C., Fisher, V. A., Coe, M. T., Lenters, J. D., Young-Molling, C., Ramankutty, N., Norman, J. M., \& Gower, S. T. (2000). Testing the performance of a dynamic global ecosystem model: Water balance, carbon balance, and vegetation structure. Global Biogeochemical Cycles, 14(3), 795-825.

Le Maitre, D. C., Thuiller, W., \& Schonegevel, L. (2008). Developing an approach to defining the potential distributions of invasive plant species: a case study of Hakea species in South Africa. Global Ecology and Biogeography, 17(5), 569584.

Liu, C., Berry, P. M., Dawson, T. P., \& Pearson, R. G. (2005). Selecting thresholds of occurrence in the prediction of species distributions. Ecography, 28(385-393.

Lobo, J. A., Jimenez-Valverde, A., \& Real, R. 2008. AUC: a misleading measure of the performance of predictive distribution models. Global Ecology and Biogeography. 17, 145-151.

Machado, A. B. M., Drummond, G. M., \& Paglia, A. P. (2008a). Livro Vermelho da Fauna Brasileira ameaçada de extinção, volume 2 (Vol. 2). Brasília-DF: MMA.

Machado, R. B., Aguiar, L. M. S., Castro, A. A. J. F., Nogueira, C. C., \& Neto, M. B. R. (2008b). Caracterização da Fauna e Flora do Cerrado. In F. Faleiro \& A. Neto (Eds.), Savanas - desafios e estratégias para o equilíbrio entre sociedade, agronegócio e recursos naturais. Planaltina- DF: Embrapa Cerrados.

Machado, R. B., Neto, M. B. R., Pereira, P. G. P., Caldas, E. F., Gonçalves, D. A., Santos, N. S., Tabor, K., \& Steininger, M. (2004). Estimativas de perda da área do Cerrado brasileiro. In C. International (Ed.), (pp. 23). Brasília-DF.

Marini, M. Â., Barbet-Massin, M., Lopes, L. E., \& Jiguet, F. (2010a). Predicting the occurrence of rare Brazilian birds with species distribution models. Journal of Ornithology, 151(4), 857-866.

Marini, M. Â., Barbet-Massin, M., Martinez, J., Prestes, N. P., \& Jiguet, F. (2010b). Applying ecological niche modelling to plan conservation actions for the Redspectacled Amazon (Amazona pretrei). Biological Conservation, 143(1), 102112.

Marmion, M., Parviainen, M., Luoto, M., Heikkinen, R. K., \& Thuiller, W. (2009). Evaluation of consensus methods in predictive species distribution modelling. Diversity and Distributions, 15(1), 59-69.

Meller, L., Cabeza, M., Pironon, S., Barbet-Massin, M., Maiorano, L., Georges, D., \& Thuiller, W. (2014). Ensemble distribution models in conservation prioritization: from consensus predictions to consensus reserve networks. Diversity \& distributions, 20(3), 309-321.

MMA. (2002). Biodiversidade brasileira: avaliação e identificação de áreas e ações prioritárias para conservação, utilização sustentável e repartição dos benefícios 
da biodiversidade nos biomas brasileiros. In SBF (Ed.), (pp. 404). Brasília- DF: MMA.

MMA. (2006). Mapa de Áreas Prioritárias do Cerrado em formato SHAPE. In. BrasíliaDF.

MMA. (2014). Portaria 444, de 17 de dezembro de 2014. In MMA (Ed.), 444 (pp. 121 126). DOU.

MMA/IBAMA. (2009). Relatório técnico de monitoramento do desmatamento no bioma Cerrado, 2002 a 2008: Dados revisados. In. Brasília-DF: MMA.

MMA/IBAMA. (2011a). Monitoramento do desmatamento nos biomas brasileiros por satélite: acordo de cooperação técnica MMA/IBAMA. Monitoramento do bioma Cerrado 2008-2009. In. Brasília-DF: MMA.

MMA/IBAMA. (2011b). Monitoramento do desmatamento nos biomas brasileiros por satélite: acordo de cooperação técnica MMA/IBAMA. Monitoramento do bioma Cerrado 2009-2010. In. Brasília-DF: MMA.

Myers, N., Mittermeier, R. A., Mittermeier, C. G., Fonseca, G. A. B., \& Kent, J. (2000). Biodiversity hotspots for conservation priorities. Nature, 403(853-858.

Oliveira-Filho, A. T., \& Ratter, J. A. (1995). A study of the origin of central brazilian forests by the analysis of plant species distribution patterns. Edinburgh Journal of Botany, 52(2), 141-194.

Oliveira, R. C. R. d., Coelho, A. S., \& Melo, F. R. (2003). Estimativa de densidade populacional de Sauá (Callicebus nigrifrons) em um fragmento de mata em regeneração, Viçosa, Minas Gerais, Brasil Neotropical Primates, 11(2), 91-93.

Paglia, A. P., Fonseca, G. A. B., Rylands, A. B., Herrmann, G., Aguiar, L. M. S., Chiarello, A. G., Leite, Y. L. R., Costa, L. P., Siciliano, S., Kierulff, M. C. M., Mendes, S. L., Tavares, V. d. C., Mittermeier, R. A., \& Patton, J. L. (2012). Lista Anotada dos Mamíferos do Brasil (2 $2^{\mathrm{a}}$ ed.). Arlington, VA: Conservation International.

Peterson, A. T., Soberón, J., Pearson, R. G., Anderson, R. P., Martinez-Meyer, E., Nakamura, M., \& Araujo, M. B. (2011). Ecological Niches and Geographic Distributions. Princeton, USA: Princeton University Press.

Phillips, S. J., Anderson, R. P., \& Schapire, R. E. (2006). Maximum entropy modeling of species geographic distributions. Ecological Modelling, 190(3-4), 231-259.

Phillips, S. J., Dudik, M., \& Schapire, R. E. (2004). A Maximum Entropy Approach to Species Distribution Modeling. In Proceedings of the 21st International Conference on Machine Learning (pp. 1-8). Banff, Canada.

Pinto, M. P., \& Grelle, C. E. V. (2009). Reserve selection and persistence: complementing the existing Atlantic Forest reserve system. Biodiversity and Conservation, 18(4), 957-968.

Pinto, M. P., Silva-Junior Jde, S., Lima, A. A., \& Grelle, C. E. (2014). Multi-scales analysis of primate diversity and protected areas at a megadiverse region. PLoS ONE, 9(8), e105205.

Polechová, J., \& Storch, D. (2008). Ecological Niche. In S. E. Jorgensen \& B. D. Fath (Eds.), Enciclopedia of Ecology (pp. 1088-1097). Oxford: Elsevier Academic Press.

Possingham, H. P., Ball, I. R., \& Andelman, S. (2000). Mathematical methods for identifying representative reserve networks. In S. Fersson \& M. Burgman (Eds.), Quantitative Methods for Conservation Biology (pp. 322): Springerlink.

Pressey, R. L., Humphries, C. J., Margules, C. R., Vane-Wright, R. I., \& Williams, P. H. (1993). Beyond opportunism key principles for systematic reserve selection. Trends in Ecology \& Evolution, 8(4), 124-128. 
Ratter, J. A., Ribeiro, J. F., \& Bridgewater, S. (1997). The Brazilian Cerrado Vegetation and Threats to its Biodiversity. Annals of Botany, 80(223-230.

Reed, K. E., \& Fleagle, J. G. (1995). Geographic and climatic control of primate diversity. Proceedings of the National Academy of Sciences, 92(7874-7876.

Roberge, J.-M., \& Angelstam, P. (2004). Usefulness of the Umbrella species concept as a conservation tool. Conservation Biology, 18(1), 76-85.

Rondinini, C., Wilson, K. A., Boitani, L., Grantham, H., \& Possingham, H. P. (2006). Tradeoffs of different types of species occurrence data for use in systematic conservation planning. Ecology Letters, 9(10), 1136-1145.

Rylands, A. B., \& Mittermeier, R. A. (2009). The Diversity of the New World Primates (Platyrrhini): An Annotated Taxonomy. In P. A. Garber, A. Estrada, J. C. BiccaMarques, E. W. Heymann \& K. B. Strier (Eds.), South American Primates: Comparative Perspectives in the Study of Behavior, Ecology, and Conservation. New York: Springer

Saccheri, I., Kuussaari, M., Kankare, M., Vikman, P., Fortelius, W., \& Hanski, I. (1998). Inbreeding and extinction in a butterfly metapopulation. Nature, 392(2), 491-494.

Salmona, Y. B. (2013). Cerrado com C ou com S? Modelagem de cenários futuros para o bioma. Unpublished Dissertação, Universidade de Brasília, Brasília.

Santana, B. E. M. M., Prado, M. R., Lessa, G., Rocha, E. C., \& Melo, F. R. (2008). Densidade, tamanho populacional e abundância dos primatas em MG. Revista Árvore, 32(6), 1109-1117.

São Bernardo, C. S., \& Galetti, M. (2004). Densidade e tamanho populacional de primatas em um fragmento florestal no sudeste do Brasil. Revista Brasileira de Zoologia, 21(4), 827-832.

Scott, J. M., Davis, F., Csuti, B., Noss, R., Butterfield, B., Anderson, H., Caicco, S., Erchia, F. D., Edwards, T. C., Ulliman, J., Anderson, H. A. L., \& Wright, R. G. (1993). Gap Analysis: a geographic approach to protection of biological diversity. Wildlife Monographs, 123(3-41.

Siqueira, M. F. d., \& Peterson, A. T. (2003). Consequences of global climate change for geographic distributions of Cerrado tree species. Biota Neotropica, 3(2), 1-14.

Soares-Filho, B., Rajão, R., Macedo, M., Carneiro, A., Costa, W., Coe, M., Rodrigues, H., \& Alencar, A. (2014). Cracking Brazil's Forest Code. Science, 344(363-364.

Soberón, J. (2007). Grinnellian and Eltonian niches and geographic distributions of species. Ecology Letters, 10(12), 1115-1123.

Soberón, J., \& Peterson, A. T. (2005). Interpretation of models of fundamental ecological niches and species' distributional areas. Biodiversity Informatics, 2(110 .

Stallings, J. R. (1985). Distribution and status of primates in Paraguay. Primate Conservation, 6(51-57.

Swets, J. A. (1988). Measuring the accuracy of diagnostic systems. Science, 240(12851293.

Thorn, J. S., Nijman, V., Smith, D., \& Nekaris, K. A. I. (2009). Ecological niche modelling as a technique for assessing threats and setting conservation priorities for Asian slow lorises (Primates:Nycticebus). Diversity and Distributions, 15(2), 289-298.

Thuiller, W., Georges, D., \& Engler, R. (2014). biomod2: Ensemble platform for species distribution modeling. . In ( $\mathrm{R}$ package version 3.1-64 ed.). 
Thuiller, W., Lafourcade, B., Engler, R., \& Araújo, M. B. (2009). BIOMOD - a platform for ensemble forecasting of species distributions. Ecography, 32(3), 369-373.

Turner, D. P., Cohen, W. B., Kennedy, R. E., Fassnacht, K. S., \& Briggs, J. M. (1999). Relationships between Leaf Area Index and Landsat TM Spectral Vegetation Indices across Three Temperate Zone Sites. Remote Sensing of Environment, 70(52-68.

UICN, WWF-Brasil, \& IPÊ. (2011). Metas de Aichi: Situação atual no Brasil. In R. Weigand Jr, D. C. da Silva \& D. d. O. e. Silva (Eds.). Brasília, DF.

Varela, S., Anderson, R. P., García-Valdés, R., \& Fernández-González, F. (2014). Environmental filters reduce the effects of sampling bias and improve predictions of ecological niche models. Ecography, no-no.

Vilanova, R., Júnior, J. d. S. e. S., Grelle, C. E. V., Marroig, G., \& CerqueirL, R. (2005). Limites climáticos e vegetacionais das distribuições de Cebus nigritus e Cebus robustus (Cebinae, Platyrrhini). Neotropical Primates, 13(1), 14-19.

Wang, Q., Adiku, S., Tenhunen, J., \& Granier, A. (2005). On the relationship of NDVI with leaf area index in a deciduous forest site. Remote Sensing of Environment, 94(2), 244-255.

Watson, J. E. M., Grantham, H. S., Wilson, K. A., \& Possingham, H. P. (2011). Systematic Conservation Planning: Past, Present and Future. In R. J. Ladle \& R. J. Whittaker (Eds.), Conservation Biogeography: Blackwell Publishing Ltd. 
Figure 1. Extent of the Cerrado, showing the anthropized areas in gray, and remainders of native vegetation in green, until the year 2010, in accordance to IBAMA MMA/IBAMA 2011b).

Figure 2. Binary maps of environmental adequacy for 15 taxa of primates.

Figure 3. Number of taxa in each cell based on the niche models for 15 primates.

Figure 4. Maps of the priority areas for the conservation of primates in the Cerrado generated utilizing occurrence points (A) and niche models (B). Red corresponds to the priority areas and green are protected areas.

Figure 5. Maps of irreplaceability considering points of occurrence (A) and niche models (B), considering the existing reserves. This value was obtained using the sum of the 1,000 priority solutions. 


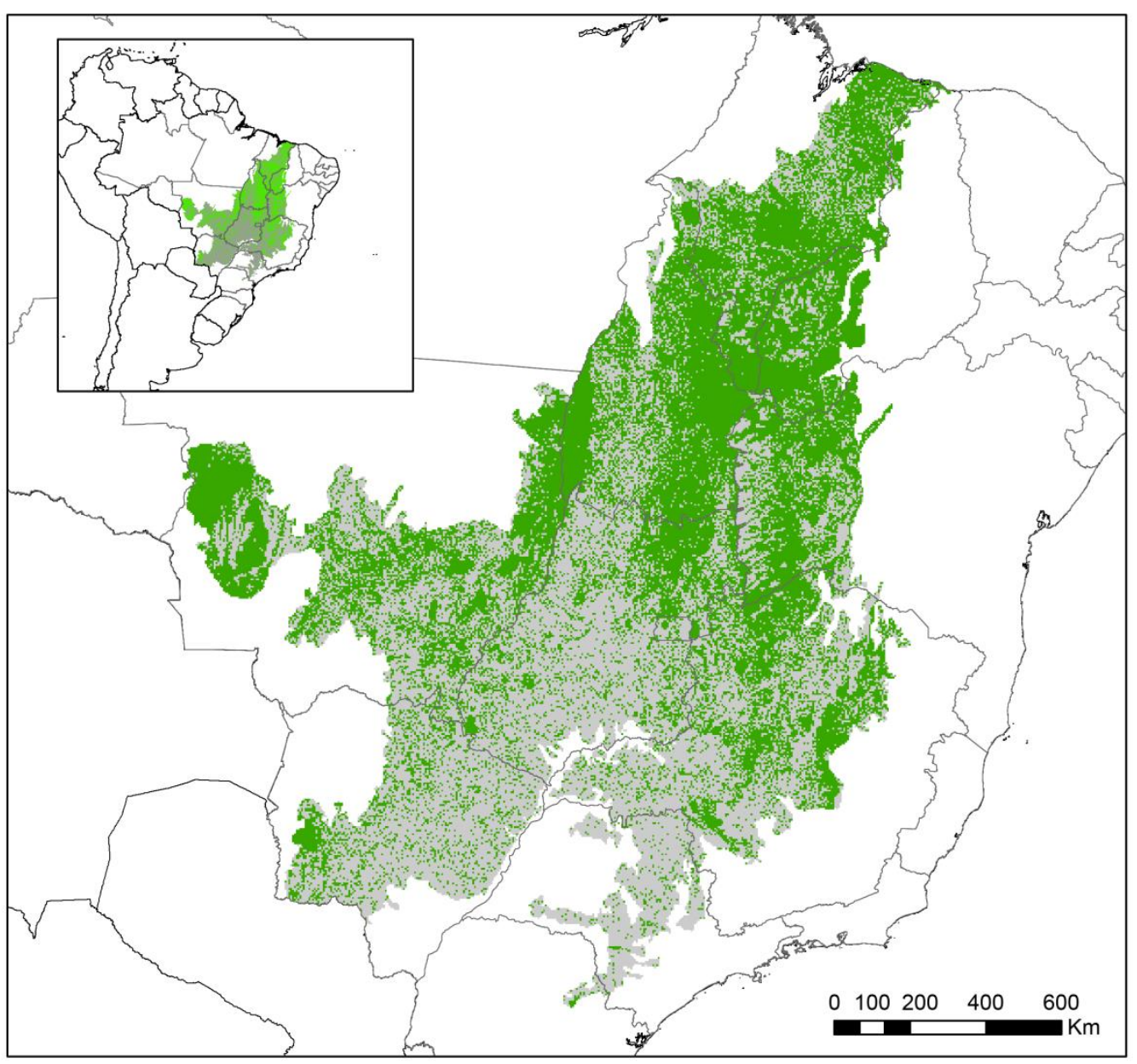




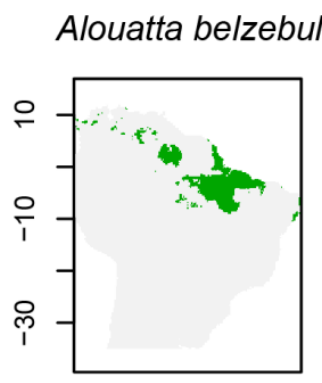

Aotus azarae

infulatus

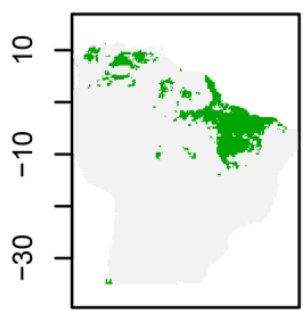

Callithrix jacchus

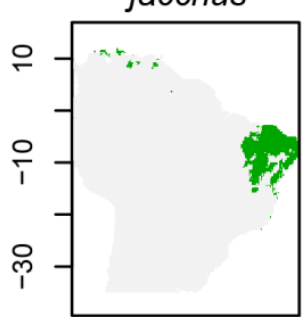

Sapajus

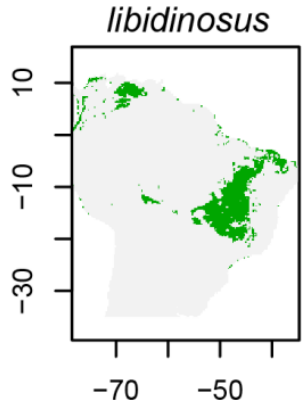

Alouatta caraya

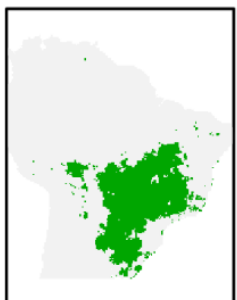

Callicebus

nigrifrons

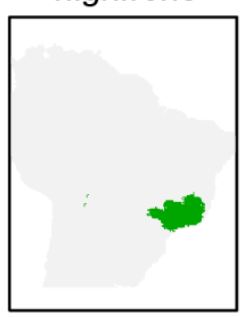

Callithrix

penicilatta

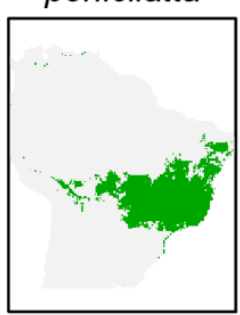

Sapajus

nigritus

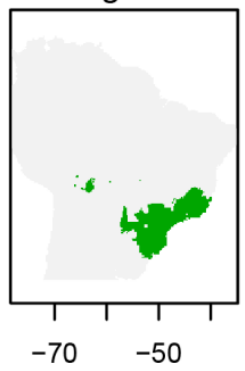

Aotus azarae

azarae

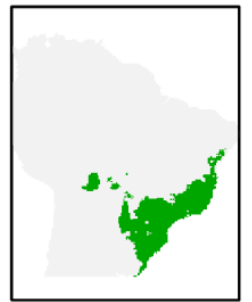

Callicebus

personatus

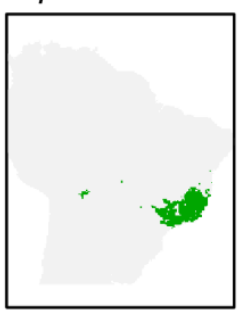

Mico

melanurus

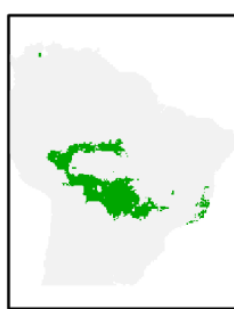

Sapajus

xanthosternos

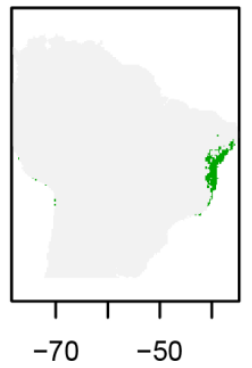

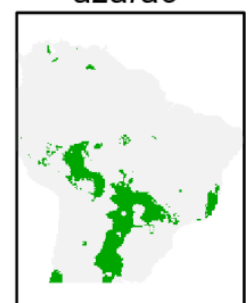

Callithrix

geoffroyi

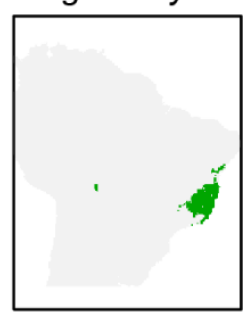

Sapajus cay

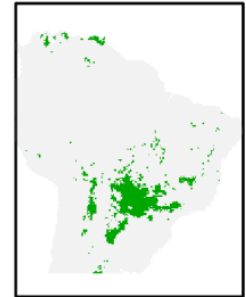




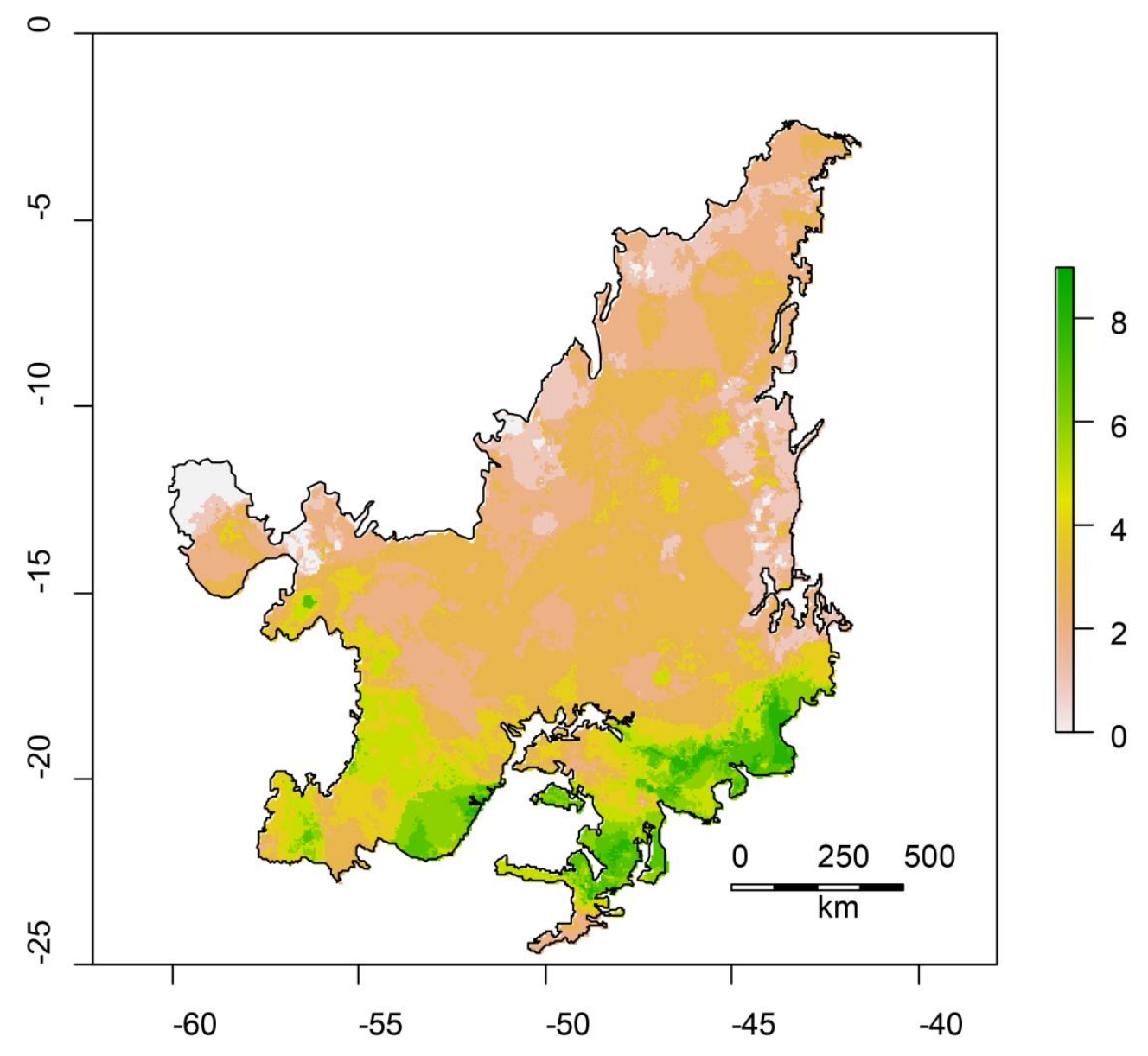




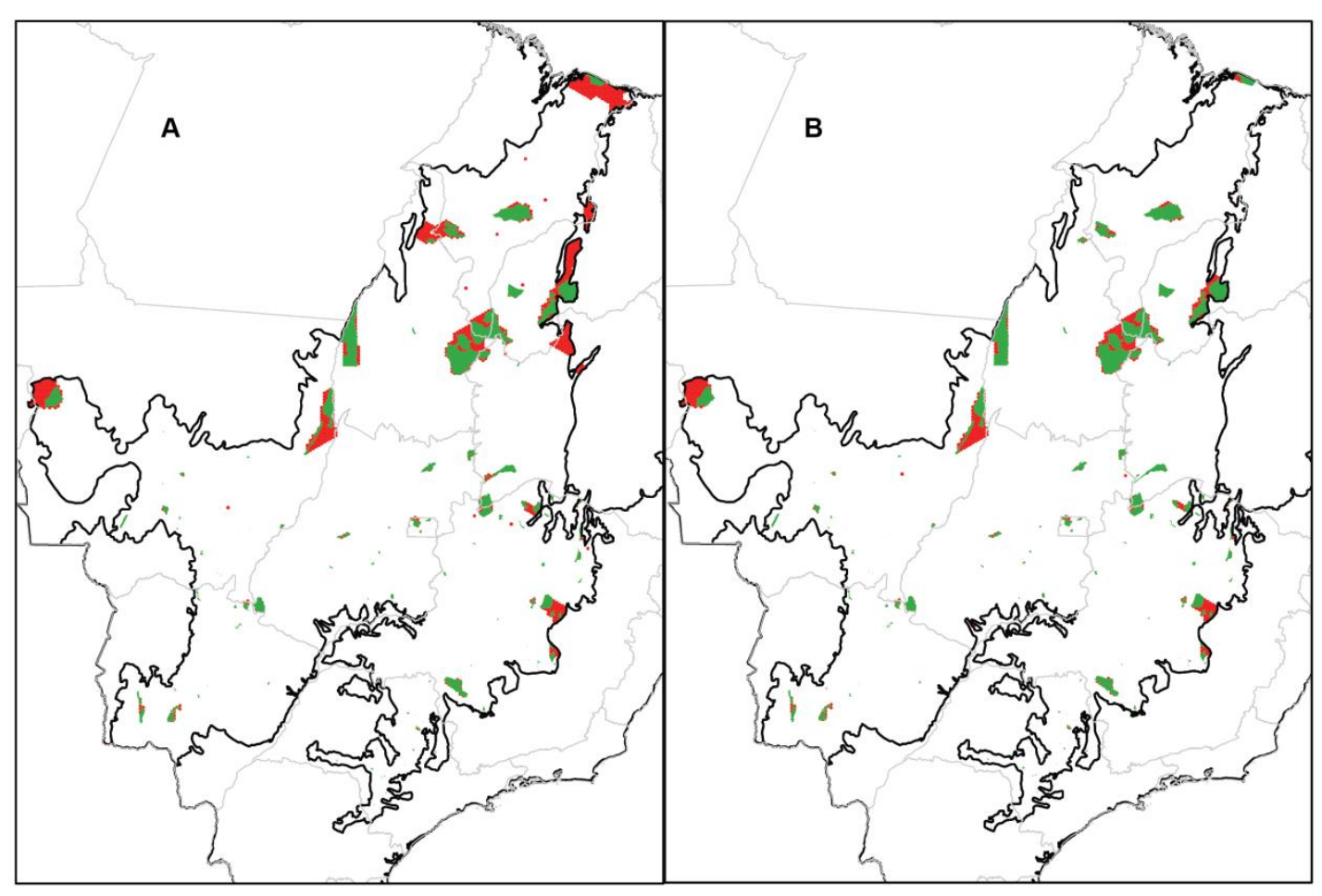




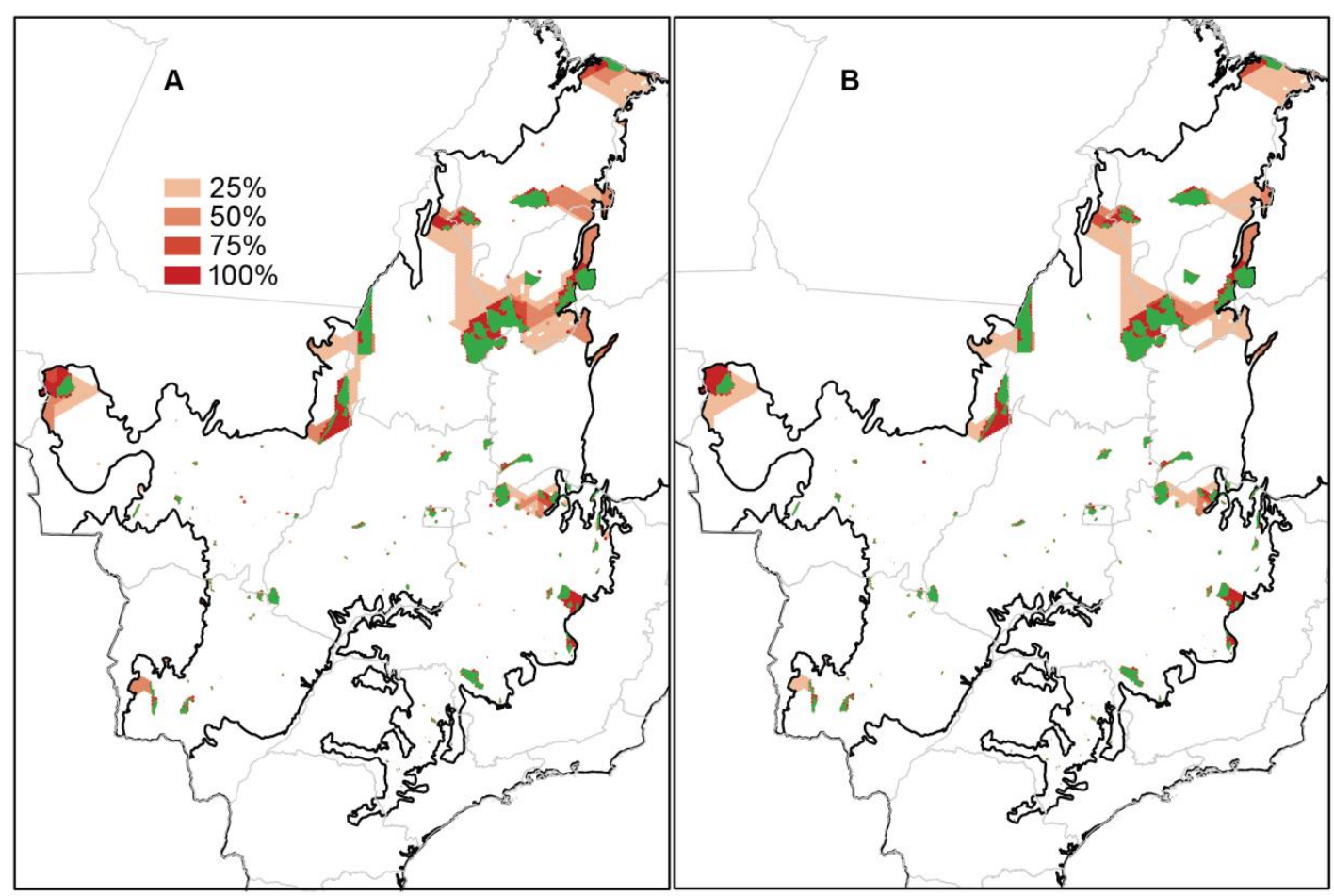




\section{CAPítulo 3}

Áreas de estabilidade climática e de endemismo para

primatas no Cerrado 


\title{
RESUMO
}

Ao longo do período quaternário a América do Sul passou por ciclos de expansão e encolhimento dos biomas graças a mudanças climáticas cíclicas. Essas mudanças provocaram alterações na distribuição das espécies de animais, fazendo com que ocorressem áreas não randômicas em que as distribuições de diversas espécies fossem congruentes, as chamadas áreas de endemismo. Essas zonas em que várias espécies se agruparam no passado são chamadas de refúgios climáticos e vários já foram apontados para primatas em áreas florestais do Brasil. Neste estudo procuramos apontar áreas de endemismo e de refúgio climático para primatas no Cerrado e compreender a contribuição de biomas vizinhos para a primatofauna do Cerrado. Para isso, coletamos a ocorrência das espécies de primatas que ocorrem no Cerrado e modelamos a distribuição potencial usando um conjunto de modelos de nicho ecológico. Os modelos foram projetados para o presente e para seis mil (meio do holoceno), 21 mil (última glaciação) e 130 mil anos (último intervalo entre glaciações) antes do presente. A distribuição das espécies foi comparada com um modelo nulo usando o teste $\mathrm{T}$ do pacote prabclus. Áreas de endemismo foram calculadas usando o software NDM somente com os pontos de ocorrência e também com pontos e modelos do presente nas escalas de $1^{\circ} \times 1^{\circ}, 2^{\circ} \times 2^{\circ}, 3^{\circ} \times 3^{\circ}$ e $4^{\circ} \times 4^{\circ}$ graus decimais. Zonas de refúgio climático para a fauna foram definidas como áreas projetadas como habitats adequados na maioria dos períodos de tempo analisados. A distribuição das espécies não é randômica, o que indica existência de uma força que moldou a distribuição dos primatas. $\mathrm{O}$ consenso das áreas de endemismo apontou quatro áreas comuns em mais de uma escala espacial, nomeadas: Transição leste, Transição norte, Transição oeste e Serras do Centro-Leste. As áreas de estabilidade climática médias foram concentradas em um corredor entre a Mata Atlântica e o Chaco. Espécies que ocupam o leste do Cerrado utilizaram refúgios em regiões de alta altitude e espécies do oeste e norte utilizaram refúgios em regiões de menor altitude. O corredor de estabilidade climática entre Mata Atlântica e Chaco indica uma possível associação passada entre a fauna desses dois biomas. Nós propomos a inclusão das áreas de estabilidade climática como prioritárias para conservação do grupo.

\begin{abstract}
In the quaternary period the South American biomes went through cycles of expansion and reduction thanks to cyclical climate changes. These changes caused changes in the distribution of various species of animals, forcing a non-random larger congruency between the ranges of various species, which are named areas of endemism. The zones in which a number of species clustered in the past are called climatic refugia and several of these have been suggested for primates in Brazil. In this study, we determined areas of endemism and climatic stability for primates of the Cerrado and the contribution of neighboring biomes for the primate fauna. In order to do that, the occurrence points of the species of primates were compiled and we modeled the potential distribution using an ensemble of ecological niche models. The models were projected to the present and to six (middle holocene), 21 (last glacial maxima) and 130 (last interglacial) thousand years before present. Species ranges were compared with a null model in T test of the package prabclus. Areas of endemism were calculated with the software NDM with
\end{abstract}


only occurrence points and also points and niche models of the present with cell size of $1^{\circ} \times 1^{\circ}, 2^{\circ} \times 2^{\circ}, 3^{\circ} \times 3^{\circ}$ and $4^{\circ} \times 4^{\circ}$ decimal degrees. Climatic refuges of the fauna were the areas projected as suitable habitat in most of the analyzed time periods. Species ranges are not randomic, which indicates the existence of some force that shaped primate ranges. The consensus of areas of endemism pointed four areas common to more than one spatial scale, which were named: East Transition, West Transition, North Transition and East-Center Hills. The mean climatic stability areas were concentrated in a corridor between the Atlantic Forest and Chaco. Species that occupies the east of the Cerrado utilized refuges in regions of high altitude and species of north and west utilizes regions of lower altitudes. The zones of climatic stability between Atlantic Forest and Chaco indicates a probable past association between the two domains. We proposo the inclusion of the areas of climatic stability as priorities to the conservation of this group.

\section{INTRODUÇÃO}

Ao longo do Quaternário, o Cerrado sofreu diversas variações na sua extensão geográfica e formato das fitofisionomias graças às mudanças climáticas ocorridas nos últimos milhares de anos. Durante as eras glaciais, a extensão das savanas se ampliava e durante os períodos mais quentes as florestas aumentavam de proporção, o que se sabe que ocorreu de forma cíclica pelo menos cinco vezes durante o período Quaternário (Salgado-Labouriau 1994). Essas grandes variações climáticas fizeram o Cerrado avançar para dentro de outros biomas em determinadas épocas e hoje podemos ver ilhas de Cerrado rodeadas pela Floresta Amazônica, como nos estados de Roraima e Rondônia (Silva and Bates, 2002).

Apesar de ser um bioma composto em sua maior parte por savanas e campos abertos, a fauna do Cerrado tem uma associação muito forte com as florestas de forma que mais da metade dos mamíferos e aves presentes no Cerrado é dependente dos ambientes florestais (Redford and Fonseca 1986, Silva and Bates 2002). Isso teria ocorrido por causa do avanço da Floresta Amazônica e Atlântica dentro do Cerrado nos períodos úmidos e quando a Caatinga e Chaco avançavam nos períodos mais secos as espécies da fauna dependentes de florestas se refugiavam nos remanescentes florestais (Silva and Bates 2002). 
A contração e expansão das formações vegetais propicia o aparecimento de áreas de congruência não randômicas na distribuição de diferentes táxons, chamadas de áreas de endemismo (Morrone 1994). Vários estudos já foram feitos tentando encontrar áreas de endemismo para certos grupos taxonômicos. No Cerrado, foram sugeridos três centros de endemismo para a distribuição de aves (Silva 1997) e dez centros para a distribuição de répteis (Nogueira et al. 2011). Para primatas amazônicos, os refúgios florestais tendem a concordar com as áreas de endemismo propostas com base nas distribuições geográficas de aves (Jones 1987, Silva and Oren 1996). Kinzey (1982) propôs áreas de endemismo para os primatas da Mata Atlântica que discorda das áreas propostas com base em dados de répteis.

A Biogeografia apresenta vários métodos diferentes de análise dos padrões de distribuição das espécies no passado (Ronquist 1997, Crisci 2001, Szumik et al. 2002, Hausdorf and Hennig 2003, Nihei 2006, Lamm and Redelings 2009). Posadas e colaboradores (2006) listam 33 métodos de análise da biogeografia histórica distribuídos em nove abordagens científicas diferentes. Um dos métodos mais usados é a análise parcimônica de endemismo (PAE), que utiliza os táxons comuns em cada área para prever a história do local mais parcimoniosa. Ela foi utilizada para primatas neotropicais por Goldani et al. (2006) que propuseram várias áreas que possuem táxons comuns, dentre elas quatro que ligam o Cerrado a outros biomas. No entanto, PAE é um método muito criticado por não utilizar informações sobre filogenia, por falhar em descobrir a história biogeográfica de sistemas que não sofreram vicariância e também por ser muito variável dependendo do tamanho das unidades de área (Brooks and van Veller 2003, Nihei 2006).

Dois métodos que tentam reconstruír áreas de agrupamentos de espécies são: NDM (eNDeMismo) (Szumik et al. 2002, Szumik and Goloboff 2004) e análise de 
elementos bióticos (Hausdorf and Hennig 2003). A análise de elementos bióticos utiliza uma abordagem baseada no modelo de vicariância, onde primeiro se testa se existe agrupamento que pode ser explicado por processos históricos de vicariância e depois se realiza uma análise de agrupamento para verificar quais grupos existem (Hausdorf 2002, Hausdorf and Hennig 2003, Hausdorf and Hennig 2004). Já a análise de endemicidade, NDM, é um algoritmo que busca distribuições de espécies similares de forma iterativa e que possui quatro critérios de otimização para selecionar as melhores áreas de amostragem (Szumik et al. 2002, Szumik and Goloboff 2004). Em estudos que analisaram o desempenho de PAE, NDM e elementos bióticos (e outros métodos com agrupamento), os algoritmos apresentaram soluções bem diferentes e variáveis, no entanto o NDM sempre se mostrou mais preciso (Carine et al. 2009, Casagranda et al. 2012).

Uma alternativa aos métodos variáveis de reconstrução da biogeografia histórica é utilizar a modelagem de nicho ecológico para reconstruir áreas de distribuição geográfica histórica das espécies através de similaridades climáticas com o presente. Os modelos de nicho ecológico (ENM) são algoritmos estatísticos que utilizam variáveis climáticas e ambientais para reconstruir o nicho de uma espécie e prever áreas em que o ambiente é favorável à existência desta (Soberón and Peterson 2005, Peterson et al. 2011, Peterson and Soberón 2012). As variáveis utilizadas são geralmente descritoras do ambiente abiótico (porque é mais difícil de ter variáveis de interação biótica em escala continental), fazendo o ENM descrever o nicho abiótico ou grineliano (Soberón 2007). Partindo da premissa que os requisitos de nicho das espécies variam lentamente (chamado de conservantismo de nicho (Peterson et al. 1999)) ENMs podem utilizar o nicho reconstruído a partir das ocorrências recentes da espécie e projetá-lo para o passado. Essa abordagem já foi usada para estudar diversas questões importantes da 
paleobiologia, como refúgios glaciais, extinções da megafauna e impactos das mudanças climáticas do passado na diversidade e distribuição das espécies (Svenning et al. 2011).

Uma aplicação dos ENMs para a descoberta de padrões biogeográficos é a reconstrução das áreas de estabilidade climática, que são as áreas que permanecem adequadas para a existência das espécies por vários períodos de tempo. As áreas de estabilidade climática já foram indicadas como áreas que concentram alta diversidade genética(Carnaval et al. 2009) e alta riqueza de espécies (Carnaval and Moritz 2008, Werneck et al. 2012) e se mostraram tão precisos quanto métodos filogeográficos para estimar os refúgios climáticos do Quaternário (Hugall et al. 2002, Waltari et al. 2007). Adicionalmente, podemos combinar áreas de endemismo e áreas de estabilidade climática como áreas de refúgio climático específicos de espécies com simpatria.

Neste capítulo buscamos identificar áreas de refúgio climático e áreas de endemismo dos primatas no Cerrado e entender como esse grupo se relaciona com biomas vizinhos. Procuramos responder: quais são as áreas de endemismo e de estabilidade climáticas para primatas do Cerrado. Buscamos saber também qual bioma vizinho teve mais contribuição para a primatofauna do Cerrado. Nossas hipóteses são as seguintes: as áreas de refúgio climático de primatas no Cerrado são semelhantes às áreas de estabilidade para a vegetação do Cerrado e o bioma que tem mais contribuição para a primatofauna do Cerrado é a Mata Atlântica. 


\section{MATERIAL E MÉTODOS}

\section{Dados de espécies}

Foi adotada a nomenclatura taxonômica utilizada em Rylands e Mittermeier (2009) e foi utilizado o gênero Sapajus ao invés de Cebus conforme recomendado por Alfaro e colaboradores (2012b). Somente foram utilizadas espécies que tinham pelo menos um ponto de ocorrência no Cerrado ou no Pantanal (por ser considerada uma área de transição entre Cerrado e outros domínios morfoclimáticos (Ab`Saber 1977)). Contudo, foram utilizados todos os pontos de ocorrência encontrados dos táxons para as modelagens, mesmo os existentes fora do Cerrado. Os seguintes táxons de primatas foram analisados como do Cerrado: Alouatta belzebul, A. caraya, A. guariba, Aotus azarae azarae, A. azarae infulatus, Callicebus donacophilus, C. nigrifons, $C$. personatus, Callithrix geoffroyi, C. jacchus, C. penicillata, Mico melanurus, Sapajus cay, S. libidinosus, S. nigritus e S. xanthosternos.

Para a construção do banco de dados de ocorrências de espécies foi utilizada a mesma base de dados gerada para o capítulo 2, composta por dados de ocorrência de museus e coleções online (principalmente GBIF e specieslink), registros de especialistas obtidos pelo Centro de Primatas Brasileiros do ICMBIO e levantamento extensivo das ocorrências georreferenciadas relatadas na literatura científica.

Os registros obtidos que não tiveram indicação das coordenadas geográficas e nem da localidade de coleta foram descartados. Aqueles que só tiverem indicação da localidade de coleta foram georreferenciados usando a ferramenta geoLoc disponível na página do specieslink ou a ferramenta GEOLocate do museu de história natural da universidade de Tulane (http://www.museum.tulane.edu/geolocate/default.html). Essas ferramentas comparam o nome das localidades de registros com o acervo de localidades 
já conhecidas presentes em várias coleções para sugerir o local mais provável daquela localidade. Foi feita uma inspeção visual nos pontos de ocorrência de cada espécie e excluídos os pontos distantes dos mapas de distribuição existentes no site All the world's primates (http://www.alltheworldsprimates.org/Maps.aspx), como forma de excluir erros no georreferenciamento ou na identificação da espécie. Pontos que estavam a menos de $20 \mathrm{~km}$, aproximadamente, uns dos outros foram filtrados para retirar um viés espacial de amostragem (Varela et al. 2014).

\section{Modelagem de Nicho Ecológico}

Foi utilizado um conjunto de variáveis ambientais que envolvem precipitação, temperatura no presente e projetadas para as mesmas variáveis há 6 mil, 21 mil e 130 mil anos antes do presente (a.p.). Esses períodos de tempo foram usados para se entender a resposta à flutuação climática antes, durante e depois da última era glacial. No presente e nos três períodos do passado foram usadas as variáveis bioclimáticas bio 1 a 7 e 10 a 17, retirando as variáveis 8, 9, 18 e 19 por conterem artefatos em uma região do mapa. As variáveis do presente foram obtidas da base de dados online Worldclim (Hijmans et al. 2005) (www.worldclim.org) que contém variáveis com diferentes relações de temperatura ou precipitação globais ao longo do ano. Após treinar os modelos nessas variáveis foram feitas projeções para o passado baseado em estimativas do projeto de intercomparações de modelagens paleoclimáticas acopladas $5^{\text {a }}$ fase (CMIP5: http://cmip-pcmdi.llnl.gov/cmip5/). As variáveis são simuladas para um período de aproximadamente 6 mil (holoceno) e 21 mil (máximo da última era glacial - LGM) anos a.p. As variáveis de 130 mil anos a.p. (último intervalo entre glaciações - LIG) foram obtidas com um modelo de clima global, um modelo dinâmico de cobertura de gelo e dados paleoclimáticos (Otto-Bliesner 2006). Novas variáveis 
foram criadas a partir da análise de componentes principais (PCA), para reduzir o número de variáveis e evitar o overfit, ajuste exagerado aos dados de ocorrência. Foram utilizados os sete primeiros eixos da PCA, que explicaram mais de $99 \%$ da variação dos dados ambientais.

Para realizar as análises de modelagem de nicho ecológico foram utilizados cinco algoritmos presentes no pacote Biomod2 (Thuiller 2003, Thuiller et al. 2009), de forma a fazer uma previsão usando um conjunto de modelos. Foi utilizada a área embaixo da curva ROC (AUC) como medida de avaliação dos modelos já que o pacote a utiliza, no entanto sabemos que é uma medida criticada (Lobo et al. 2008). Para selecionar os melhores modelos foi feita uma modelagem inicial com os pontos e escolhidos os cinco algoritmos que apresentaram uma maior AUC para os dados, pois o desempenho dos algoritmos varia para cada caso (Qiao et al. 2015). É preferível usar o consenso de um conjunto de modelos a usar apenas um, pois o consenso gera um resultado mais preciso por reduzir as incertezas específicas de cada modelo (Araújo et al. 2005, Marmion et al. 2009), assim como utilizar algoritmos bem ajustados de acordo com as métricas de avaliação (Buisson et al. 2010). Os algoritmos selecionados foram: (1) modelo aditivo generalizado (GAM); (2) Modelo impulsionado generalizado (GBM); (3) redes neurais artificiais (ANN); (4) floresta randômica (RF) e (5) Máxima entropia (Maxent) (Philips et. al. 2004). Detalhes dos métodos dos algoritmos de 1 a 4 podem ser encontrados em um livro específico (Hastie et al. 2009).

Mesmo sem ter dados confiáveis de ausência das espécies é vantajoso utilizar pseudo ausências porque as predições de modelos que somente utilizam presenças são menos precisas (Elith et al. 2006). Pseudo ausências são pontos selecionados em áreas que não possuem registros de ocorrência para simular ausência para os algoritmos. Utilizamos um algoritmo para gerar pseudo-ausências disponível no pacote Biomod2, 
método este que seleciona pontos considerados como ausência potencial da espécieem um algoritmo de somente presença, o envelope de superfície de distribuição (SRE). Outros estudos já utilizaram essa opção do Biomod2 com bons resultados (Le Maitre et al. 2008, Marini et al. 2010b). Somente os modelos com AUC/ROC maior que 0,8 foram selecionados para produzir um mapa de consenso. Foi utilizada a média do valor de cada pixel dos modelos para gerar a adequabilidade climática da espécie àquele local no modelo de consenso. Para transformar os resultados dos modelos em mapas de distribuição foram cortados os valores de adequabilidade ambiental de cada pixel seguindo limiar que maximiza o valor da True Skill Statistics (TSS) (Allouche et al. 2006).

\section{Áreas de endemismo e vicariância}

Para testar se as espécies de primatas possuem distribuições agrupadas e estruturadas utilizamos o teste de agrupamento proposto por Hausdorf e Hennig (Hausdorf 2002, Hausdorf and Hennig 2003, Hausdorf and Hennig 2004). Essa análise verifica a hipótese que a distribuição das espécies é diferente do esperado ao acaso para analisar se existe um agrupamento não randômico das distribuições, o que indica que houve processos geográficos de estruturação da biota no passado. O esperado ao acaso, no método, foi obtido com a construção de um modelo nulo com 1000 repetições. Os dados observados e os esperados através do modelo nulo foram comparados com a estatística T, que é a razão entre a soma das $25 \%$ menores distâncias de Kulczynski com as 25\% maiores na distribuições (Hausdorf \& Hennig 2003). Esse teste avalia se as distâncias entre grupos de ocorrências das espécies são maiores do que as distâncias dentro dos grupos. Essa análise foi implementada no pacote Prabclus (Hennig and Hausdorf 2015) para o software R (versão x64 3.1.2). 
Após confirmar o agrupamento na distribuição das espécies foi utilizado o programa NDM/ VNDM (Szumik et al. 2002, Szumik and Goloboff 2004) para reconstruir as áreas de endemismo para o grupo. Esse programa utiliza quatro critérios de otimização para reconstruir áreas de endemismo e indica um grau de endemicidade para a espécie baseado no critério utilizado e na quantidade de células presentes dentro de cada área (Szumik and Goloboff 2004). Essa ferramenta permite incluir os resultados da modelagem de nicho ecológico como presença assumida (Szumik and Goloboff 2004). Esse programa foi considerado o melhor método para reconstrução de áreas de endemismo, em comparação com métodos de agrupamento e PAE (Carine et al. 2009, Casagranda et al. 2012).

Fizemos a análise de endemicidade (NDM) em quatro escalas espaciais: $1^{\circ} \times 1^{\circ}$, $2^{\circ} \times 2^{\circ}, 3^{\circ} \times 3^{\circ}$ e $4^{\circ} \times 4^{\circ}$ graus geográficos. Fizemos desta forma para identificar áreas de maior concentração de espécies sem perder a existência de padrões dependentes da escala. Foram consideradas as áreas em uma abordagem que utilizou somente pontos de ocorrência e outra que utilizou pontos e modelos. Para tanto foram geradas duas matrizes para cada escala, uma que continha os valores: 0 (ausência), 1 (presença observada) e 2 (presença assumida por modelos) e outra somente com 0 e 1.

Foram usados os parâmetros de seleção de apenas áreas com duas ou mais espécies restritas, escore de endemismo maior do que 1,0 e utilizada a opção edge proportion, que faz com que se contabilize as células vizinhas das áreas para o cálculo do índice de endemicidade. O agrupamento inicial foi resumido por meio do consenso flexível, considerando semelhantes aquelas áreas que possuíam mais de $50 \%$ de semelhança na composição de espécies. Foram utilizados 50 réplicas para cada matriz analisada. 


\section{RESULTADOS}

Em geral, observamos uma maior quantidade de espécies previstas em um corredor entre Mata Atlântica e o Chaco em todos os períodos de tempo (figura 1). Esse corredor de adequabilidade ambiental é formado por áreas fortemente e outras mais fracamente representadas no presente e no LIG (130 mil anos a.p.), por áreas de menor riqueza geral no Holoceno e por uma área maior e de mais alta densidade de espécies durante o LGM (21 mil anos a.p.). Segundo os modelos gerados, durante o Holoceno houve maior concentração de adequabilidade climática no norte do Cerrado (fig. 1B) e no LIG ocorreu uma maior adequabilidade no litoral do nordeste brasileiro (fig. 1D). 
A

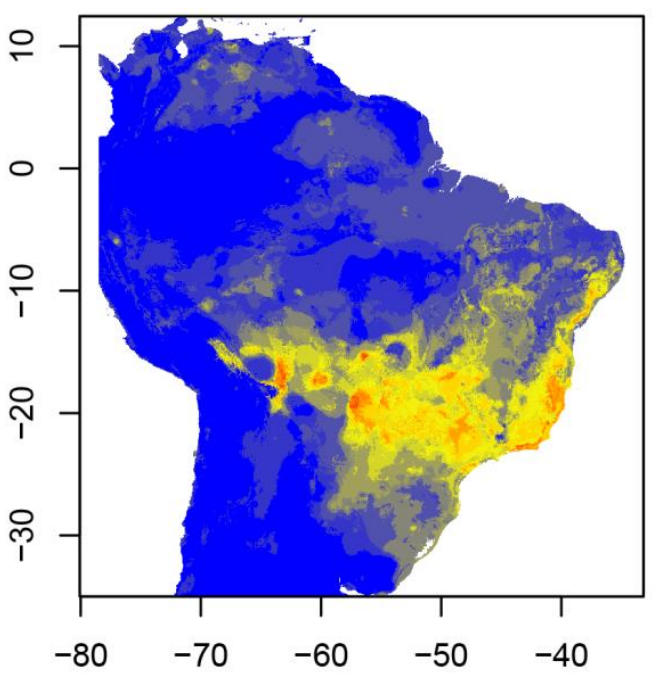

C Última glaciação - 21k

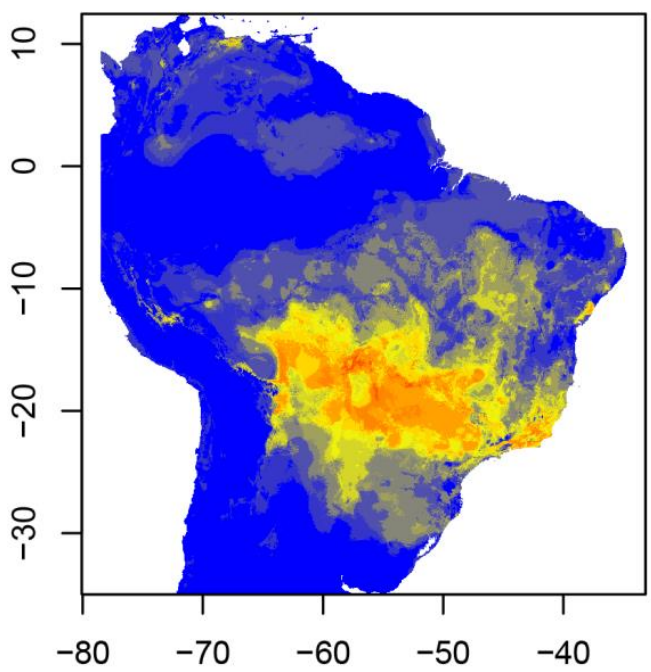

B Holoceno - $6 k$

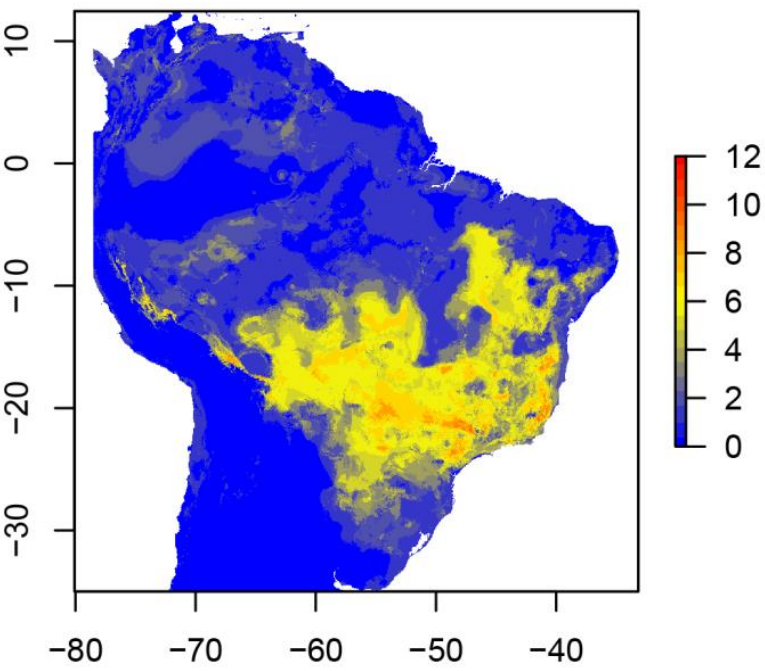

D Ültimo intervalo entre glaciações - 130k

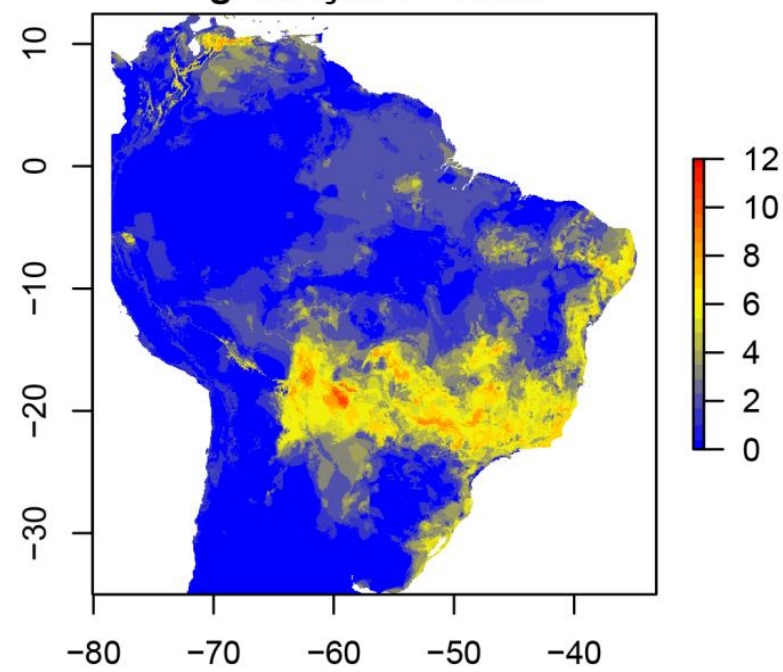

Figura 1. Mapas de riqueza das espécies de primatas do Cerrado no presente (A),

Holoceno médio (6 mil anos)(B), última glaciação (21 mil anos) (C) e último período inter-glacial (130 mil anos) (D).

Existe uma maior associação com áreas ao sul do Cerrado formando um largo corredor de zonas de habitats adequados entre a Mata Atlântica, o Cerrado e o Chaco (Figura 2). Também verificou-se segundo os modelos, que existe uma alta adequabilidade climática em habitats presentes na Mata Atlântica do Sudeste brasileiro. 
A alta adequabilidade climática fora do Cerrado indica que as espécies de primatas saíram da área de distribuição do domínio em alguns períodos de tempo, principalmente durante o LGM (figura 1). Alouatta belzebul ainda apresenta um padrão de distribuição disjunta que apresentou aproximações e afastamentos dos habitats adequados ao longo $\operatorname{dos}$ anos

(figura

$3)$.

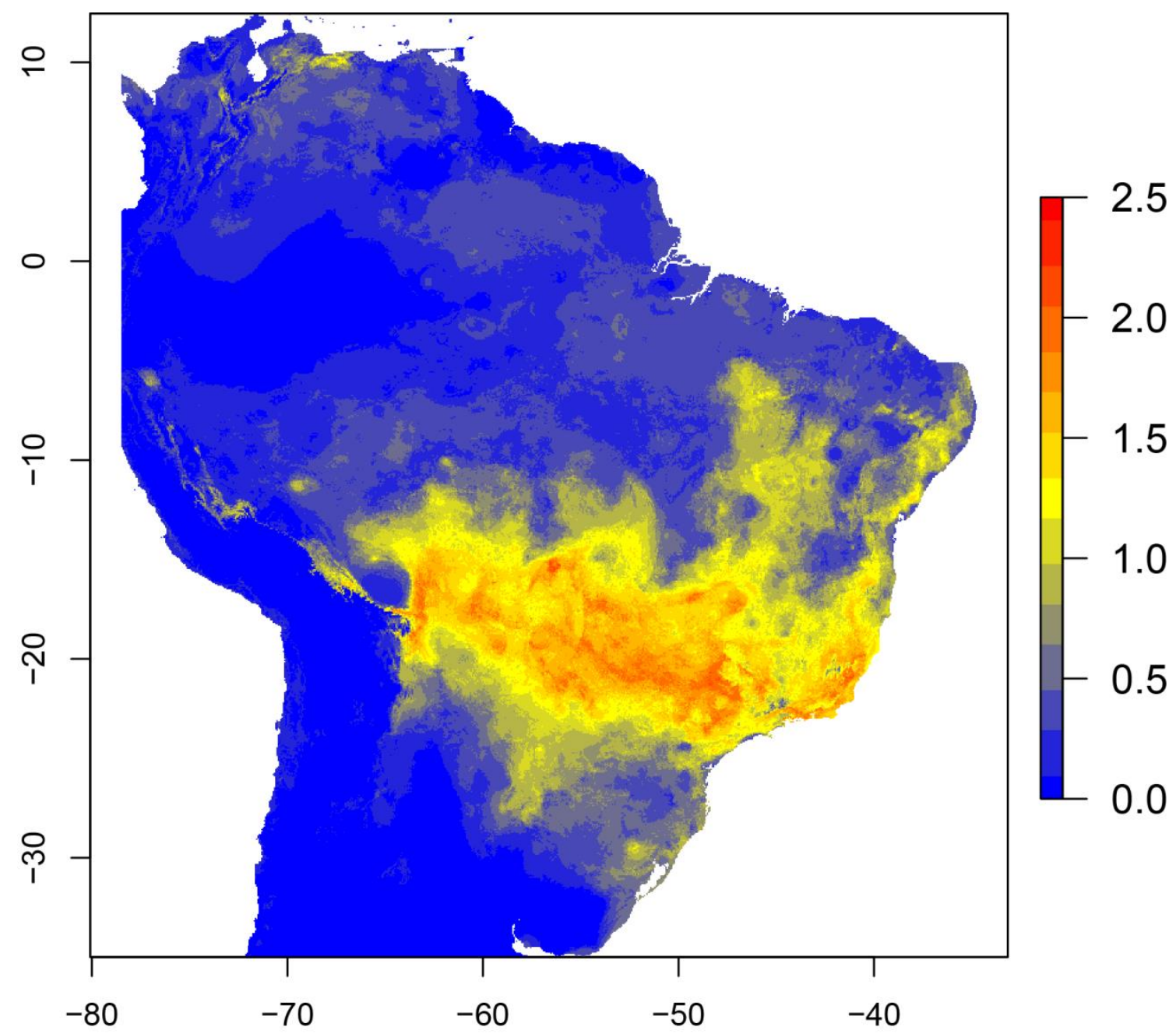

Figura 2. Adequabilidades climáticas históricas obtidas pela soma das projeções dos cenários do presente, 6 mil, 21 mil e 130 mil anos para cada espécie e calculando a média entre todas as espécies. Valores mais altos indicam áreas que foram selecionadas como presença em maior número de cenários. 

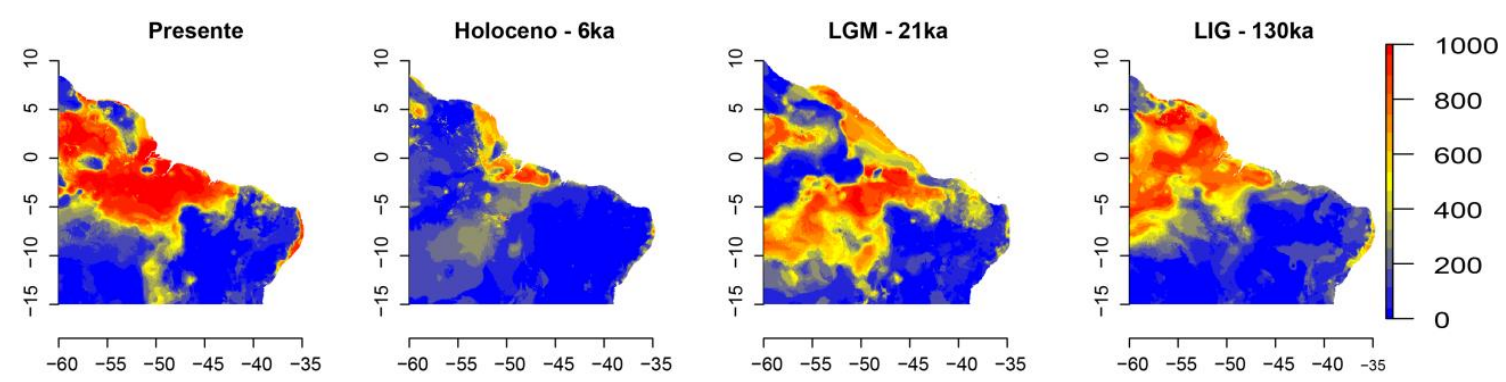

Figura 3. Foco na distribuição potencial histórica de Alouatta belzebul mostrando as mudanças nas duas áreas de distribuição disjunta ao longo do tempo.

A distribuição dos pontos de ocorrência das espécies se mostrou agrupado em relação ao modelo nulo em todas as escalas de análise $\left(1^{\circ} \times 1^{\circ}: \mathrm{T}=0,361\right.$, Nulo=0,452, $\mathrm{p}=0,01 ; 2^{\mathrm{o}} \times 2^{\mathrm{o}}: \mathrm{T}=0,47$, Nulo $=0,70, \mathrm{p}<0,01 ; 3^{\mathrm{o}} \times 3^{\mathrm{o}}: \mathrm{T}=0,40$, Nulo $=0,67, \mathrm{p}<0,01 ; 4^{\mathrm{o}} \times$ $4^{\mathrm{o}}: \mathrm{T}=0,35$, Nulo=0,63, $\mathrm{p}<0,01$ ) (tabela 1). No entanto, não houve agrupamento da distribuição quando foram considerados apenas os modelos de distribuição potencial (Tabela 1). 
Tabela 1. Valores da estatística $\mathrm{T}$ para agrupamento da distribuição das espécies calculado com um modelo nulo.

\begin{tabular}{llll}
\hline & $\mathrm{T}$ & Nulo & $\mathrm{P}$ \\
\hline Pontos $-1^{\mathbf{o}}$ & 0,36 & 0,45 & 0,01 \\
Pontos $-2^{\text {o }}$ & 0,47 & 0,70 & $<0,01$ \\
Pontos $-3^{\text {o }}$ & 0,40 & 0,67 & $<0,01$ \\
Pontos $-4^{\text {o }}$ & 0,35 & 0,63 & $<0,01$ \\
Modelos $-1^{\text {o }}$ & 0,99 & 0,65 & 1,00 \\
Modelos $-2^{\text {o }}$ & 0,92 & 0,70 & 1,00 \\
Modelos $-3^{\text {o }}$ & 0,97 & 0,60 & 1,00 \\
Modelos $-4^{\text {o }}$ & 0,99 & 0,65 & 1,00 \\
\hline
\end{tabular}

$\mathrm{Na}$ abordagem somente com pontos de ocorrência não houve nenhuma área de endemismo encontrada na escala de $1^{\circ} \times 1^{\circ}$ e a escala que apresentou maior número de áreas foi a com quadrículas de $3^{\circ} \times 3^{\circ}$ (5 áreas) (Tabela 2). Foram apontadas quatro principais áreas que foram comuns em mais de uma escala. A Transição leste é representada por seis espécies que ocorrem na fronteira do Cerrado com a Mata Atlântica ou com a Caatinga (Figura 4- A, D rosa e F): Callicebus nigrifrons, Callicebus personatus, Callithrix geoffroyi, Callithrix jacchus, Sapajus libidinosus e Sapajus xanthosternos. A área Serras do Centro-Leste é formada por seis espécies que se concentram no sudeste do domínio (Figura 4 - B, E e G): Alouatta guariba, Callicebus nigrifrons, Callicebus personatus, Callithrix penicillata, Sapajus libidinosus e Sapajus nigritus. A área Transição Oeste é formada pelas espécies que ocorrem na divisa entre Cerrado, Pantanal e Chaco (Figura 4-D azul e H rosa): Aotus azarae azarae, Callicebus donacophillus, Mico melanurus e Sapajus cay. Por último, a área 
Transição Norte, que é formada por duas espécies que ocorrem no Cerrado e na Amazônia (Figura 4- C azul e H azul): Alouatta belzebul e Aotus azarae infulatus.

Tabela 2. Resultados da análise de endemicidade baseada em pontos.

\begin{tabular}{llll}
\hline Grade & $\mathrm{N}^{\mathbf{o}}$ de Sets & $\mathrm{N}^{\mathbf{o}}$ de áreas de endemismo & Mínima e máxima pontuação \\
\hline $1^{\mathbf{0}} \times 1^{\mathbf{o}}$ & - & - & - \\
$2^{\mathbf{o}} \times 2^{\mathbf{o}}$ & 2 & 2 & $1,43-1,68$ \\
$3^{\mathbf{0}} \times 3^{\mathbf{o}}$ & 7 & 5 & $1,64-2,62$ \\
$4^{\mathbf{o}} \times 4^{\mathbf{o}}$ & 8 & 4 & $1,36-3,51$ \\
\hline
\end{tabular}


A

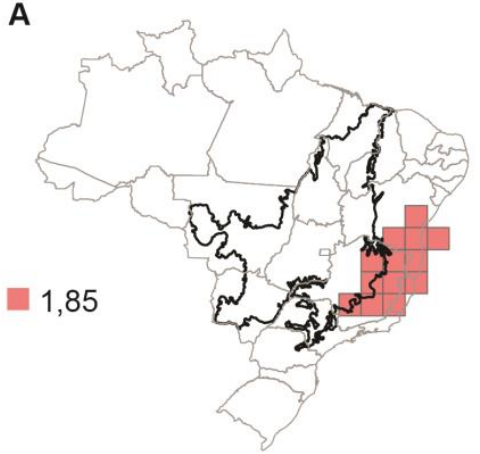

D

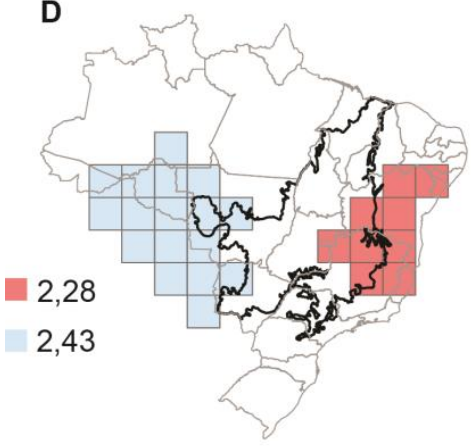

G

3,51

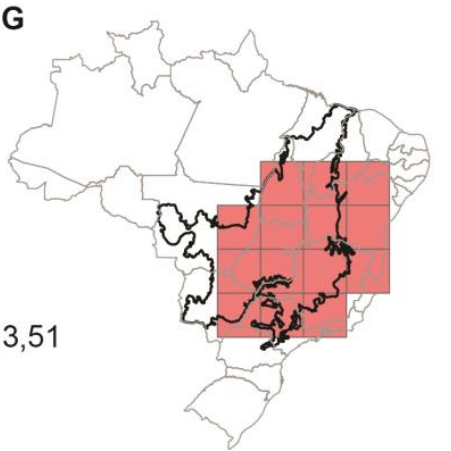

B

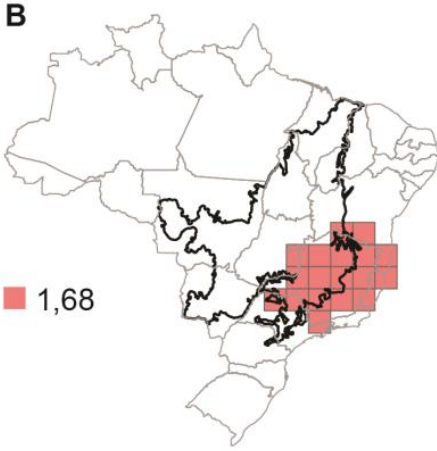

E

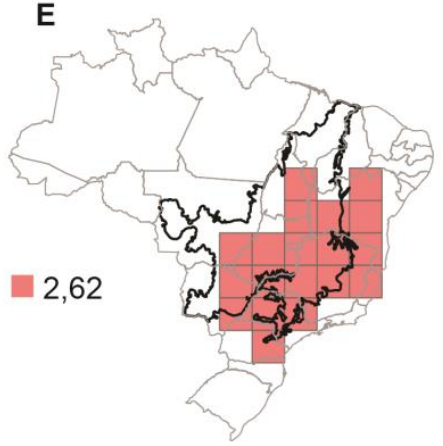

H

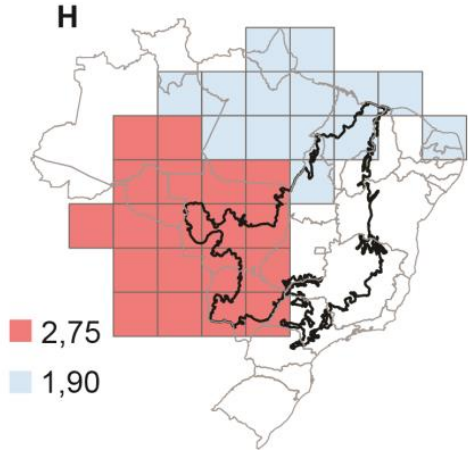

C
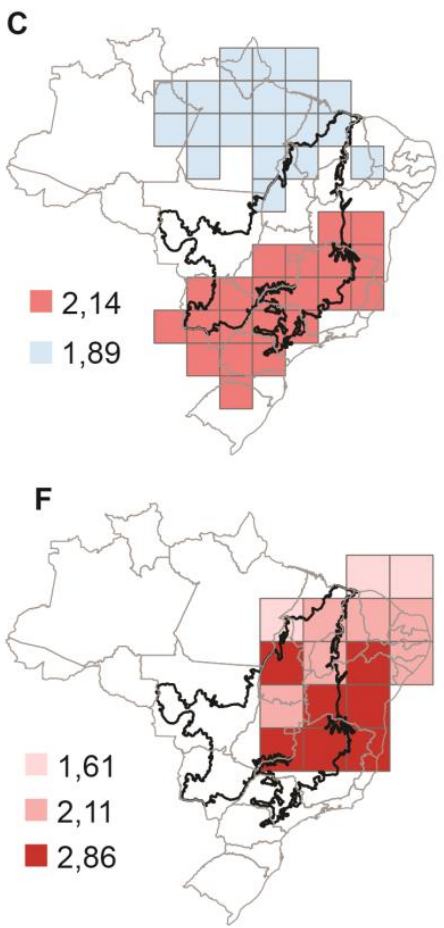

Figura 4. Áreas de endemismo geradas com o método de otimização NDM com tamanho de célula de $2^{\circ} \times 2^{\circ}\left(\mathrm{A}\right.$ e B), $3^{\circ} \times 3^{\circ}\left(\mathrm{C}, \mathrm{D}\right.$ e E) e $4^{\circ} \times 4^{\circ}(\mathrm{F}, \mathrm{G}$ e H). Áreas similares foram fundidas em um consenso das espécies presentes na área.

$\mathrm{Na}$ abordagem por pontos e modelos de distribuição também não foi encontrado nenhum agrupamento de espécies na escala de $1^{\circ} \times 1^{\circ}$ e as quadrículas de $4^{\circ} \times 4^{\circ}$ foram aquelas que geraram maior número de áreas (5 áreas) (Tabela 3). De modo geral, os valores de endemicidade foram menores na abordagem com modelos de distribuição, com uma variação de 1,121 a 2,936 contra 1,428 - 3,515 quando foram considerados somente pontos (Tabelas 2 e 3). Foram identificados quatro padrões principais de 
distribuição, um localizado ao redor das serras e planaltos do centro-leste brasileiro (Figura 5- A, F e J), outro na transição com biomas do leste (Figura 5- B, E e I), um terceiro na transição com os do oeste (Figura 5- C e H) e o quarto no centro do Brasil (Figura 5- G e K).

A partir da delimitação de áreas em diferentes escalas e juntando em consenso aquelas com distribuição e espécies semelhantes selecionamos quatro áreas de endemismo para o Cerrado, sendo elas: Serras do Centro-Leste, Transição leste, Transição oeste e Transição norte (Figura 6). Pelo número de áreas de endemismo e representação das espécies em cada uma delas, pode-se observar que a Mata Atlântica é o bioma adjacente mais influente para a primatofauna do Cerrado.

Tabela 3. Resultados da análise de endemicidade baseada em pontos e modelos.

\begin{tabular}{|c|c|c|c|}
\hline Grade & $\mathrm{N}^{\mathrm{o}}$ de Sets & $\mathrm{N}^{\mathrm{o}}$ de áreas de endemismo & Mínima e máxima pontuação \\
\hline $1^{\circ} \times 1^{\circ}$ & - & - & - \\
\hline $2^{\circ} \times 2^{\circ}$ & 3 & 2 & $1,12-1,73$ \\
\hline $3^{\circ} \times 3^{\circ}$ & 7 & 4 & $1,60-2,14$ \\
\hline $4^{\circ} \times 4^{\circ}$ & 9 & 5 & $1,25-2,94$ \\
\hline
\end{tabular}



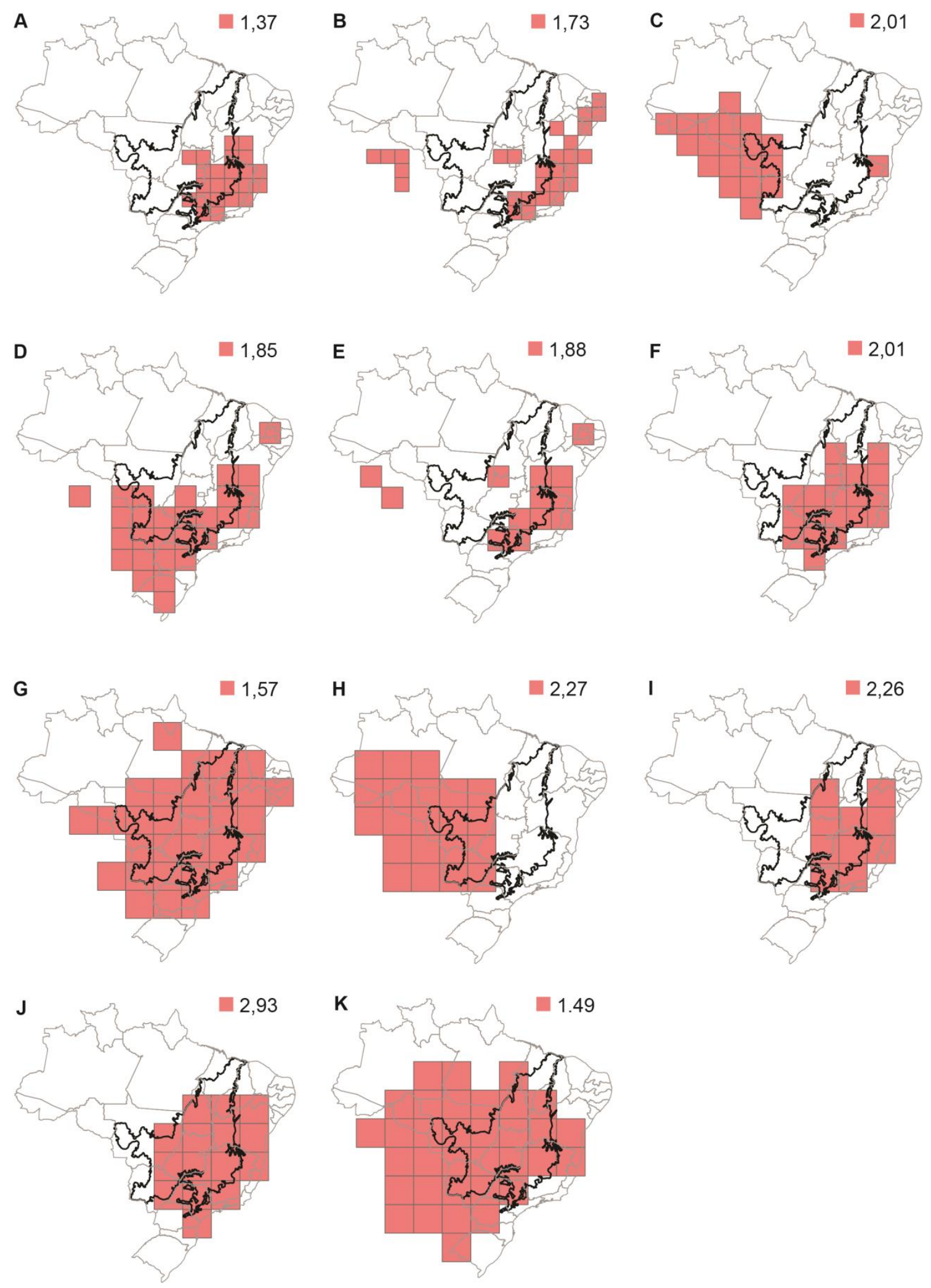

Figura 5. Áreas de endemismo geradas com o programa NDM utilizando pontos de ocorrência e modelos de distribuição de espécies com tamanhos de célula $2^{\circ} \times 2^{\circ}$ (A e B), $3^{\circ} \times 3^{\circ}\left(\mathrm{C}, \mathrm{D}, \mathrm{E}\right.$ e F ) e $4^{\circ} \times 4^{\circ}(\mathrm{G}, \mathrm{H}, \mathrm{I}, \mathrm{J}$ e K$)$. Valores correspondem ao índice de endemicidade. 

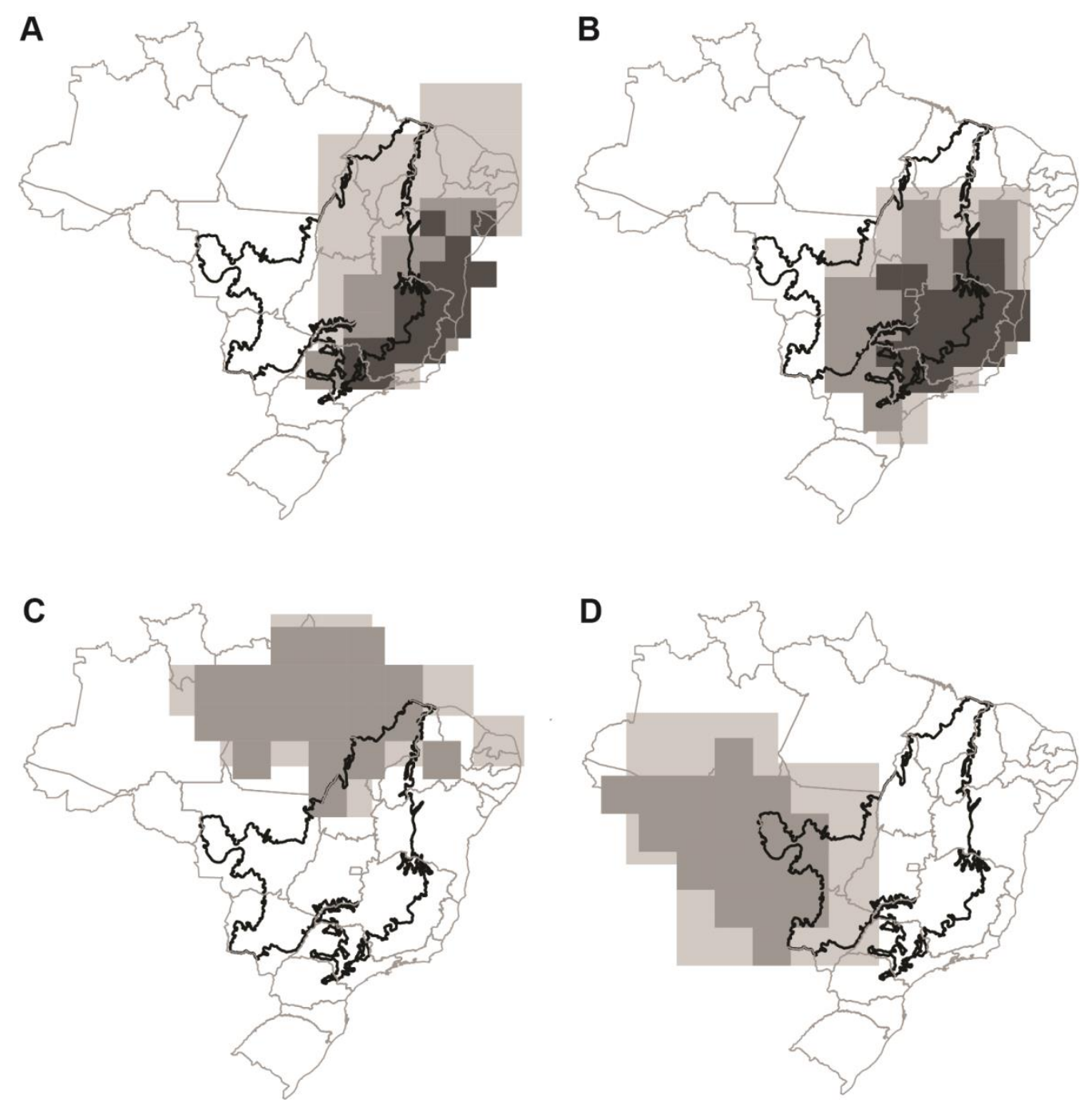

Figura 6. Mapa mostrando a delimitação das áreas de endemismo baseado na distribuição de primatas que habitam o Cerrado. (A) Transição leste, (B) Serras do Centro-Leste, (C) Transição norte e (D) Transição oeste.

Considerando somente as espécies que contribuem para as áreas de endemismo o padrão das zonas de estabilidade climáticas se torna levemente diferente. A maior concentração de áreas de estabilidade para as espécies da transição leste e da Serras do Centro-Leste se dá no sul do domínio do Cerrado, com as principais zonas sendo no sul de Minas, norte de São Paulo e no sul de Goiás (figura 7). Para as espécies da transição norte as zonas de estabilidade se concentram principalmente no Maranhão e Pará. As 
espécies da transição oeste encontram refúgio ao longo do tempo em uma área que engloba o sudoeste do Cerrado, o Pantanal e uma parte do Chaco Boliviano (Figura 7).
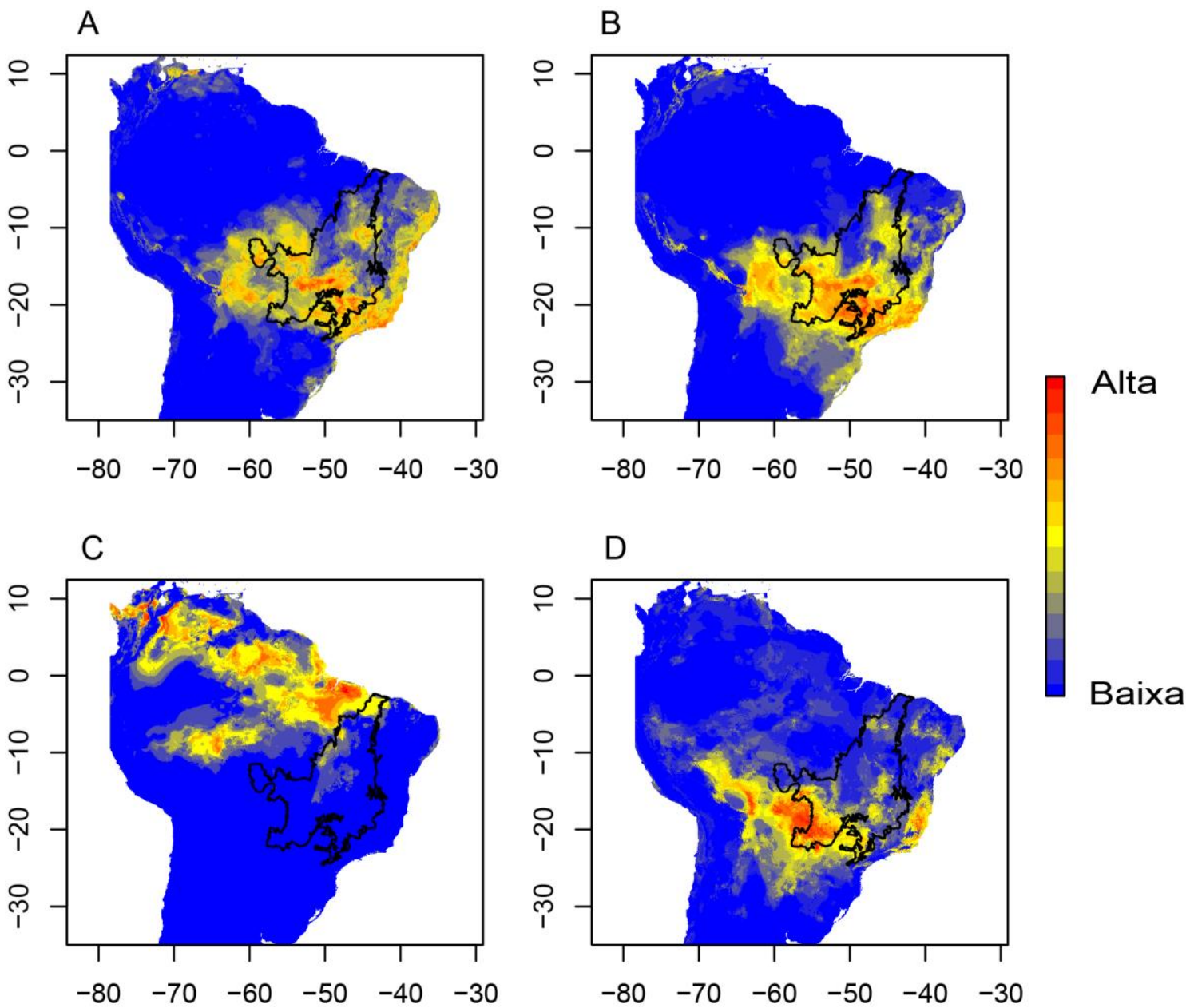

Figura 7. Mapas de estabilidade climática histórica média ao longo do tempo considerando somente as espécies que contribuem para a delimitação das áreas de endemismo calculadas na Transição leste (A), Serras do Centro-Leste (B), Transição norte (C) e Transição oeste (D).

\section{DISCUSSÃO}

\section{Áreas de endemismo}

No nosso conhecimento é a primeira vez que as áreas de endemismo foram analisadas focando em primatas que ocorrem no Cerrado. Encontramos associação de 
grande parte das espécies com as áreas de transição ao leste do domínio e um menor número estava associado à transição oeste e norte.A distribuição das espécies de primatas que ocorrem no Cerrado se mostrou agrupada em relação ao modelo nulo gerado com 1000 repetições, quando consideramos somente os pontos de ocorrência. O agrupamento indica a existência de padrões históricos de vicariância que moldaram as distribuições das espécies existentes através de barreiras (Hausdorf 2002, Hausdorf and Hennig 2003, Hausdorf and Hennig 2004). Na abordagem por modelos de nicho não foi possível verificar agrupamento das distribuições, talvez porque os modelos tenderam a extrapolar para áreas ambientalmente similares e geograficamente distantes, mascarando o agrupamento das áreas de presença real. As barreiras históricas no processo de vicariância no Cerrado parecem ser flexíveis, mudando conforme as mudanças climáticas do Quaternário que causaram expansões e retrações da vegetação (Werneck 2011).

As áreas de endemismo apontadas pelo método de otimização foram quatro: Transição leste, Transição oeste, Transição norte e Serras do Centro-Leste. Das áreas de endemismo apontadas, a Serras do Centro-Leste é a única que está localizada predominantemente no Cerrado, pois tem seu núcleo na cadeia do Espinhaço e se expande para o planalto central do Brasil. A cadeia do Espinhaço é uma região rica em biodiversidade e representa áreas de endemismo para outros grupos (Silva 1997, Silva and Bates 2002, Nogueira et al. 2011). A área Serras do Centro-Leste está representada pelas espécies: Alouatta guariba, Callicebus nigrifrons, Callicebus personatus, Callithrix penicillata, Sapajus libidinosus e Sapajus nigritus.

A área de transição leste é formada por espécies cujas distribuições englobam o Cerrado e a Mata Atlântica ou o Cerrado e a Caatinga. Esta área é composta por espécies adaptadas a uma zona intermediária entre um domínio morfoclimático e outro. 
É formado por Callicebus nigrifrons, Callicebus personatus, Callithrix geoffroyi, Callithrix jacchus, Sapajus libidinosus e Sapajus xanthosternos. A teoria mais aceita para a chegada de Platyrrhini no Novo Mundo é através de uma rota de migração direta partindo da África, colonizando a região que hoje possui Mata Atlântica a aproximadamente 37 milhões de anos a.p., idade estimada pela divergência genética entre os grupos (Kay 2015, Kiesling et al. 2015). O registro fóssil de primatas neotropicais mais antigo foi encontrado na Bolívia e sua idade foi estimada em 26 milhões de anos a.p. (Kay 2015). Portanto, as comunidades de primatas da Mata Atlântica são as mais antigas e possuem um histórico de interação com áreas mais secas no centro do continente desde o início da colonização das Américas.

A área de transição oeste é uma área que engloba algumas espécies que estão associadas a uma área de transição entre o Cerrado, o Pantanal e o Chaco. As espécies da área incluem Aotus azarae azarae, Callicebus donacophillus, Mico melanurus e Sapajus cay. Já a área de transição norte, é formada apenas por duas espécies que ocorrem na divisa entre Cerrado e Amazônia: Alouatta belzebul e Aotus azarae infulatus. Os registros fósseis presentes na região da área de transição oeste são os mais antigos do continente e os presentes na região Amazônica são em torno de 17 milhões de anos mais recentes (Kay 2015).

As entradas das espécies para o Cerrado ocorreram em um período de tempo relativamente recente, possivelmente ocorreram eventos de ocupação e outros de extinção na área gerando uma ocupação intermitente, enquanto a ocupação foi contínua em áreas mais úmidas (Lynch Alfaro et al. 2015). Exemplos dessa ocupação mais recente é a diversificação dos Sapajus modernos para as áreas mais secas do continente, que ocorreu há apenas 400 mil anos (Lynch Alfaro et al. 2012), a diversificação de Callithrix para Caatinga e Cerrado ocorrido há 1 milhão de anos (Buckner et al. 2014) 
e a formação das três espécies de Alouatta ocorrida há 4 milhões de anos atrás (CortésOrtiz et al. 2003, Lynch Alfaro et al. 2015). Essa colonização recente indica que a ocupação do Cerrado foi mais lenta, por causa do acúmulo de adaptações necessárias para se viver nesse ambiente, como a gomivoria (Buckner et al. 2014). Desta forma, as áreas de endemismo que têm menos tempo de colonizadas apresentam um número menor de táxons capazes de interagir com este ambiente, sendo que a Mata Atlântica tem o conjunto mais antigo seguido do Chaco e da Amazônia (Kay 2015).

\section{Refúgios de estabilidade climática}

As áreas de estabilidade climática apontadas para todas as espécies representam um grande corredor que liga os domínios leste e oeste, passando pelo centro-sul do Cerrado. A estabilidade climática aponta uma região que teve o clima favorável para a ocorrência em mais de um período de tempo e indica regiões com maior diversidade genética e riqueza de espécies (Hugall et al. 2002, Carnaval and Moritz 2008, Carnaval et al. 2009, Werneck et al. 2012). As áreas de maior probabilidade de presença histórica concordam com zonas de alta altitude, com pontos de alta estabilidade ocorrendo na serra da Canastra, na serra do Caiapó, chapada dos Guimarães, planalto central goiano, planalto central da bacia do rio Paraná e uma área próxima à cordilheira dos Andes. Isso indica que as espécies em geral utilizaram as áreas de maior altitude para se refugiar das mudanças climáticas ocorridas nos últimos 130 mil anos. Esse padrão também é encontrado para aves florestais da Mata Atlântica (Silva 1996).

As zonas de estabilidade climática apontadas para primatas diferem das encontradas como estáveis para todo o Cerrado (Werneck et al. 2012). Para o Cerrado, as áreas mais estáveis estão presentes no centro norte, com zonas de refúgio apontadas 
no Maranhão, serra geral de Goiás e no centro-oeste, próximo ao rio Araguaia (Werneck et al. 2012). O padrão geral de distribuição das zonas de estabilidade encontrado está mais concordante com a distribuição das zonas de florestas tropicais sazonalmente secas (SDTFs) definição ampla e das zonas de Mata Atlântica, no sentido que elas também apresentam uma faixa de adequabilidade oeste-leste atravessando pelo sul do Cerrado e São Paulo (Carnaval and Moritz 2008, Werneck et al. 2011). Como a diferença entre as zonas de SDTFs definição ampla e restrita é a inclusão das florestas semideciduais brasileiras (Werneck et al. 2011), que também estão presentes na definição da Mata Atlântica (Carnaval and Moritz 2008), essa foi provavelmente a formação vegetal utilizada predominantemente por primatas do Cerrado como refúgio climático.

Considerando que as espécies utilizaram refúgios diferentes dependendo da comunidade a que pertenciam e onde estavam localizadas, vimos que o padrão de refúgios para as espécies varia conforme a área de endemismo em que elas se encontram. Para as espécies da área de endemismo Serras do Centro-Leste e Transição leste o padrão é bastante parecido, com zonas de estabilidade na Serra da Canastra, Serra dos Pirineus, Serra dos Caiapós e próximo ao Rio Grande em São Paulo. As zonas nas serras de Goiás e Mato Grosso são consideradas como cerrado em 3 dos 4 períodos de tempo analisados por Werneck (Werneck et al. 2012), indicando uma estabilidade média como Cerrado. As zonas presentes em Minas e Goiás são também citadas no trabalho de Carnaval e Moritz (2008) como zona de estabilidade de Mata Atlântica. Essa diferença na estabilidade da vegetação na área indica a preferência por habitats de transição para as espécies associadas com essa zona.

Já para as espécies das áreas de transição oeste e norte, apontamos áreas de alta estabilidade principalmente em terrenos de planície úmida. Uma zona importante para a área Transição oeste é situada na planície do Pantanal e entorno, adentrando no Chaco e 
no planalto da bacia do rio Paraná. Para a área de transição norte a zona de mais alta estabilidade histórica se encontra na planície em torno do rio Gurupi entre o Maranhão e o Pará. Essa zona é coincidente com o refúgio Belém do trabalho de Haffer (1969). Para essas duas áreas de endemismo as espécies se refugiaram em áreas predominantemente fora do domínio morfoclimático do Cerrado, provavelmente porque são comunidades cuja colonização na região foi mais recente e ainda não tiveram tempo para evoluir adaptações para o ambiente sazonalmente seco do Cerrado (Kay 2015, Lynch Alfaro et al. 2015).

Essa característica de espécies que ocupam a Mata Atlântica adentrarem o Cerrado através de áreas de alta altitude e espécies da Amazônia através de planícies e depressões também é encontrada em aves florestais (Silva 1996). Neste grupo animal, as espécies da Mata Atlântica também entram significativamente mais no interior do bioma, inclusive chegando em áreas na divisa com o Chaco (Silva 1996). 


\section{Cerrado e biomas vizinhos}

O Cerrado ocupa posição central no continente sul-americano e no Brasil e apresenta conexão com cinco outros biomas, sendo eles: Amazônia e Mata Atlântica de formações florestais, Caatinga e Chaco de formações abertas e Pantanal, que possui características similares ao Cerrado, só que com grandes áreas alagáveis (Ab’Saber, 1977). É de se imaginar que o Cerrado, com sua heterogeneidade de fitofisionomias serviria de corredor tanto para as formações abertas quanto para as formações florestais (através das matas de galeria e cerradões) (Oliveira-Filho and Ratter 1995). No presente estudo, o que encontramos foi a existência de um corredor de adequabilidade climática entre a Mata Atlântica (formação florestal) e o Chaco (formação mais aberta).

Essa conexão entre Mata Atlântica e Chaco contrasta com a informação de vários trabalhos que relatam a existência de similaridades na biota das duas grandes formações florestais. A análise da distribuição de árvores revelou que boa parte das espécies arbóreas de florestas do Cerrado estão distribuídas ao longo das duas diagonais e as árvores de formações florestais se distribuíam ao longo das matas de galeria de sudeste a noroeste de forma a ligar a Mata Atlântica e a Amazônia (Oliveira-Filho and Ratter 1995). A distribuição das espécies de aves da Mata Atlântica e da Amazônia foi analisada por Silva (Silva 1996) para descobrir o quanto e como elas adentravam no Cerrado. As espécies da Amazônia entram por áreas de baixa altitude (menos que $500 \mathrm{~m}$ ) e só entram até $250 \mathrm{~km}$ da borda do Cerrado, já as de Mata Atlântica entram por áreas de alta altitude (mais que 500m) e entram até mais de $1000 \mathrm{~km}$ na região (Silva 1996). No entanto, as aves já utilizaram áreas mais secas para cruzar entre Amazônia e Mata Atlântica diversas vezes no passado com a rota mais recente atravessando pelo norte e pelo sul da Caatinga e norte do Cerrado (Willis 1992, Batalha-Filho et al. 2012). Os pequenos mamíferos possuem uma grande similaridade entre as duas formações 
florestais de forma que, em geral, as populações da Mata Atlântica não aparecem diferentes geneticamente das encontradas na Amazônia, se encontram na verdade aninhadas dentro de clados da Amazônia ou de populações mais centrais (Costa 2003).

Um exemplo existente de animal que migrou da Mata Atlântica para a Amazônia é Alouatta belzebul. Este primata possui distribuição disjunta entre a Amazônia e a Mata Atlântica nordestina e os modelos de nicho apontam que ele pode ter atravessado a Caatinga em direção a Amazônia durante o LGM (figura 3). As flutuações climáticas durante o Quaternário fizeram as duas populações estarem afastadas no LIG, se aproximarem com alguns pontos pequenos mais próximos, se afastarem novamente no holoceno e atualmente se aproximarem novamente (figura 3 ). Esse processo de afastamento e aproximação dos habitats não chegou a manter as duas populaçãos em contato em nenhum período, porém pode ter permitido a existência de fluxo gênico através de dispersão nas manchas de habitat próximas. Essa rota de travessia concorda em parte com o proposto por Cortés-Ortiz (2003) porque a rota de travessia é através da Caatinga em épocas que a vegetação estava mais favorável, no entanto a época do LGM (21 ka) é mais favorável do que a do Holoceno (6 ka) conforme proposto. Um grande fóssil de primata extinto encontrado na Caatinga baiana há aproximadamente 10 a 12 mil anos (Vivo 1997) aponta a existência de um clima mais úmido e vegetação florestal na região, além de maior número de espécies que não sobreviveu às flutuações climáticas.

Nosso estudo mostra que a maior parte das espécies de primatas utilizaram áreas de refúgio próximas à Mata Atlântica e ao Chaco no sul do Cerrado e inclusive em áreas externas à distribuição atual do Cerrado. Essas áreas apresentaram maior adequabilidade climática e ocuparam uma área maior durante o LGM (figura 1). Isso mostra a importância da Mata Atlântica e dos Chacos para a diversidade de primatas, 
principalmente da Mata Atlântica, que concentra maior número de espécies e áreas de endemismos. Esse padrão é parcialmente semelhante ao encontrado em aves, onde a diversidade central é acrescida pelo sul através da Mata Atlântica (Silva 1996), entretanto a contribuição da Amazônia para a diversidade é pequena.

Batalha-Filho e colaboradores (2012) relatam uma rota que aves da Mata Atlântica teriam utilizado há mais de 7 milhões de anos a.p. para se deslocar através do Cerrado e por áreas da Bolívia e Paraguai para chegar à Amazônia. Essa é uma passagem muito mais antiga do que o período de tempo analisado, no entanto existe um primata que pode ter aproveitado essa passagem no Cerrado mais recentemente: o Sapajus cay. Este animal se diversificou de um ancestral comum a Sapajus nigritus (associada à transição Cerrado e Mata Atlântica) há aproximadamente 400 mil anos atrás (Lynch Alfaro et al. 2012) e atualmente habita áreas do Chaco.

\section{Considerações finais}

Este é o primeiro estudo que examinou as áreas de endemismo e história biogeográfica de primatas que ocorrem no Cerrado. É necessário maior número de trabalhos para examinar o padrão biogeográfico para um período de tempo maior, para identificar como foi a resposta a outras mudanças climáticas do Quaternário. Como áreas de refúgio são correlacionadas com riqueza e diversidade genética (Carnaval et al. 2009, Werneck et al. 2012) assim como áreas de endemismo são importantes para a conservação (Silva et al. 2004), é recomendado incluir essas áreas como prioridades para a conservação do grupo de primatas para a região em estudo. 


\section{REFERÊNCIAS BIBLIOGRÁFICAS}

Ab’Saber, A. N. 1977. Os domínios morfoclimáticos na América do Sul. Geomorfologia 52:1-23.

Alfaro, J. W. L., J. D. E. S. E. Silva, and A. B. Rylands. 2012. How Different Are Robust and Gracile Capuchin Monkeys? An Argument for the Use of Sapajus and Cebus. American Journal of Primatology 74:273-286.

Allouche, O., A. Tsoar, and R. Kadmon. 2006. Assessing the accuracy of species distribution models: prevalence, kappa and the true skill statistic (TSS). Journal of Applied Ecology 43:1223-1232.

Araújo, M. B., R. J. Whittaker, R. J. Ladle, and M. Erhard. 2005. Reducing uncertainty in projections of extinction risk from climate change. Global Ecology and Biogeography 14:529-538.

Batalha-Filho, H., J. Fjeldså, P.-H. Fabre, and C. Y. Miyaki. 2012. Connections between the Atlantic and the Amazonian forest avifaunas represent distinct historical events. Journal of Ornithology 154:41-50.

Brooks, D. R. and M. G. P. van Veller. 2003. Critique of PAE as a method of historical biogeography. Journal of Biogeography 30:819-825.

Buckner, J. C., J. W. Lynch Alfaro, A. B. Rylands, and M. E. Alfaro. 2014. Biogeography of the marmosets and tamarins (Callitrichidae). Molecular phylogenetics and evolution 82 Pt B:413-425.

Buisson, L., W. Thuiller, N. Casajus, S. Lek, and G. Grenouillet. 2010. Uncertainty in ensemble forecasting of species distribution. Global Change Biology 16:11451157.

Carine, M. A., C. J. Humphries, I. R. Guma, J. A. Reyes-Betancort, and A. Santos Guerra. 2009. Areas and algorithms: evaluating numerical approaches for the delimitation of areas of endemism in the Canary Islands archipelago. Journal of Biogeography 36:593-611.

Carnaval, A. C., M. J. Hickerson, C. F. B. Haddad, M. T. Rodrigues, and C. Moritz. 2009. Stability predicts genetic diversity in the Brazilian Atlantic Forest hotspot. Science 323:785-789.

Carnaval, A. C. and C. Moritz. 2008. Historical climate modelling predicts patterns of current biodiversity in the Brazilian Atlantic forest. Journal of Biogeography 35:1187-1201.

Casagranda, M. D., L. Taher, and C. a. Szumik. 2012. Endemicity analysis, parsimony and biotic elements: A formal comparison using hypothetical distributions. Cladistics 28:645-654.

Cortés-Ortiz, L., E. Bermingham, C. Rico, E. Rodríguez-Luna, I. Sampaio, and M. Ruiz-García. 2003. Molecular systematics and biogeography of the Neotropical monkey genus, Alouatta. Molecular phylogenetics and evolution 26:64-81.

Costa, L. P. 2003. The historical bridge between the Amazon and the Atlantic Forest of Brazil: A study of molecular phylogeography with small mammals. Journal of Biogeography 30:71-86. 
Crisci, J. V. 2001. The voice of historical biogeography. Journal of Biogeography 28:157-168.

Elith, J., C. H. Graham, R. P. Anderson, M. Dudık, S. Ferrier, A. Guisan, R. J. Hijmans, F. Huettmann, J. R. Leathwick, A. Lehmann, J. Li, L. G. Lohmann, B. A. Loiselle, G. Manion, C. Moritz, M. Nakamura, Y. Nakazawa, J. M. Overton, A. T. Peterson, S. J. Phillips, K. Richardson, R. Scachetti-pereira, R. E. Schapire, J. Soberón, S. Williams, M. S. Wisz, and N. E. Zimmermann. 2006. Novel methods improve prediction of species' distributions from occurrence data. Ecography 29:129-151.

Goldani, A., G. S. Carvalho, and J. C. Bicca-Marques. 2006. Distribution patterns of neotropical primates (Platyrrhini) based on Parsimony Analysis of Endemicity. Brazilian journal of biology 66:61-74.

Hastie, T., R. Tibshirani, and J. Friedman. 2009. Elements of Statistical Learning - Data Mining, Inference and Prediction. 2nd edition. Springer, California, US.

Hausdorf, B. 2002. Units in Biogeography. Systematic Biology 51:648-652.

Hausdorf, B. and C. Hennig. 2003. Biotic Element Analysis in Biogeography. Systematic Biology 52:717-723.

Hausdorf, B. and C. Hennig. 2004. Does vicariance shape biotas Biogeographical tests of the vicariance model in the north-west European land snail fauna. Journal of Biogeography 31:1751-1757.

Hennig, C. and B. Hausdorf. 2015. prabclus: Functions for Clustering of Presenceabsence, abundance and multilocus genetic data.

Hijmans, R. J., S. E. Cameron, J. L. Parra, P. G. Jones, and A. Jarvis. 2005. Very high resolution interpolated climate surfaces for global land areas. International Journal of Climatology 25:1965-1978.

Hugall, A., C. Moritz, A. Moussalli, and J. Stanisic. 2002. Reconciling paleodistribution models and comparative phylogeography in the Wet Tropics rainforest land snail Gnarosophia bellendenkerensis (Brazier 1875). Proceedings of the National Academy of Sciences of the United States of America 99:6112-6117.

Jones, C. B. 1987. Evidence supporting the Pleistocene Forest Refuge hypothesis for primates. Biotropica 19:373-375.

Kay, R. F. 2015. Biogeography in deep time - What do phylogenetics, geology, and paleoclimate tell us about early platyrrhine evolution? Molecular phylogenetics and evolution 82 Pt B:358-374.

Kiesling, N. M. J., S. V. Yi, K. Xu, F. Gianluca Sperone, and D. E. Wildman. 2015. The tempo and mode of New World monkey evolution and biogeography in the context of phylogenomic analysis. Molecular phylogenetics and evolution $82 \mathbf{P t}$ B:386-399.

Kinzey, W. G. 1982. Distribution of Primates and forest refuges. Pages 455-482 in G. T. Prance, editor. Biological Diversification in the Tropics. Columbia University Press, New York, NY.

Lamm, K. S. and B. D. Redelings. 2009. Reconstructing ancestral ranges in historical biogeography: properties and prospects. Journal of Systematics and Evolution 47:369-382.

Le Maitre, D. C., W. Thuiller, and L. Schonegevel. 2008. Developing an approach to defining the potential distributions of invasive plant species: a case study ofHakeaspecies in South Africa. Global Ecology and Biogeography 17:569-584.

Lobo, J. A., A. Jimenez-Valverde and R. Real. 2008. AUC: a misleading measure of the performance of predictive distribution models. Global Ecology and Biogeography. 17: 145-151. 
Lynch Alfaro, J. W., J. P. Boubli, L. E. Olson, A. Di Fiore, B. Wilson, G. A. GutiérrezEspeleta, K. L. Chiou, M. Schulte, S. Neitzel, V. Ross, D. Schwochow, M. T. T. Nguyen, I. Farias, C. H. Janson, and M. E. Alfaro. 2012. Explosive Pleistocene range expansion leads to widespread Amazonian sympatry between robust and gracile capuchin monkeys. Journal of Biogeography 39:272-288.

Lynch Alfaro, J. W., L. Cortes-Ortiz, A. Di Fiore, and J. P. Boubli. 2015. Special issue: Comparative biogeography of Neotropical primates. Molecular phylogenetics and evolution 82 Pt B:518-529.

Marini, M. Â., M. Barbet-Massin, J. Martinez, N. P. Prestes, and F. Jiguet. 2010. Applying ecological niche modelling to plan conservation actions for the Redspectacled Amazon (Amazona pretrei). Biological Conservation 143:102-112.

Marmion, M., M. Parviainen, M. Luoto, R. K. Heikkinen, and W. Thuiller. 2009. Evaluation of consensus methods in predictive species distribution modelling. Diversity and Distributions 15:59-69.

Morrone, J. J. 1994. On the identification of areas of endemism. Systematic Biology 43:438-441.

Nihei, S. S. 2006. Misconceptions about parsimony analysis of endemicity. Journal of Biogeography 33:2099-2106.

Nogueira, C., S. Ribeiro, G. C. Costa, and G. R. Colli. 2011. Vicariance and endemism in a Neotropical savanna hotspot: distribution patterns of Cerrado squamate reptiles. Journal of Biogeography 38:1907-1922.

Oliveira-Filho, A. T. and J. A. Ratter. 1995. A study of the origin of central brazilian forests by the analysis of plant species distribution patterns. Edinburgh Journal of Botany 52:141-194.

Otto-Bliesner, B. L. 2006. Simulating Arctic Climate Warmth and Icefield Retreat in the Last Interglaciation. Science 311:1751-1753.

Peterson, A. T. and J. Soberón. 2012. Species Distribution Modeling and Ecological Niche Modeling: Getting the Concepts Right. Natureza \& Conservação 10:102107.

Peterson, A. T., J. Soberón, R. G. Pearson, R. P. Anderson, E. Martinez-Meyer, M. Nakamura, and M. B. Araujo. 2011. Ecological Niches and Geographic Distributions. Princeton University Press, Princeton, USA.

Peterson, A. T., J. Soberon, and V. Sánchez-Cordero. 1999. Conservatism of ecological niche in Evolutionary Time. Nature 285:1265-1267.

Posadas, P., J. V. Crisci, and L. Katinas. 2006. Historical biogeography: A review of its basic concepts and critical issues. Journal of Arid Environments 66:389-403.

Qiao, H., J. Soberón, and T. A. Peterson. 2015. No silver bullets in correlative ecological niche modeling: Insights from testings among many potential algorithms for niche estimation. Methods in Ecology and Evolution.

Redford, K. H. and G. A. B. Fonseca. 1986. The role of Gallery Forests in the Zoogeography of the Cerrado' s non-volant mammalian fauna. Biotropica 18:126-135.

Ronquist, F. 1997. Dispersal-Vicariance Analysis: a new approach to the quantification of Historical Biogeography. Systematic Biology 46:195-203.

Rylands, A. B. and R. A. Mittermeier. 2009. The Diversity of the New World Primates (Platyrrhini): An Annotated Taxonomy.in P. A. Garber, A. Estrada, J. C. BiccaMarques, E. W. Heymann, and K. B. Strier, editors. South American Primates: Comparative Perspectives in the Study of Behavior, Ecology, and Conservation. Springer New York. 
Salgado-Labouriau, M. L. 1994. História Ecológica da Terra. Editora Edgard Blucher Ltda., São Paulo - SP Brazil.

Silva, J. M. C. d. 1996. Distribution of amazonian and atlantic birds in gallery forests of the Cerrado region, South America. Ornitologia Neotropical 7:1-18.

Silva, J. M. C. d. 1997. Endemic bird species and conservation in the Cerrado Region, South America. Biodiversity and Conservation 6:435-450.

Silva, J. M. C. d. and J. M. Bates. 2002. Biogeographic patterns and conservation in the South American Cerrado: A tropical savanna hotspot. BioScience 52:225-234.

Silva, J. M. C. d. and D. C. Oren. 1996. Application of parsimony analysis of endemicity in Amazonian biogeography: an example with primates. Biological Journal of the Linnean Society 59:427-437.

Silva, J. M. C. d., M. C. d. Sousa, and C. H. M. Castelletti. 2004. Areas of endemism for passerine birds in the Atlantic forest, South America. Global Ecology and Biogeography 13:85-92.

Soberón, J. 2007. Grinnellian and Eltonian niches and geographic distributions of species. Ecology Letters 10:1115-1123.

Soberón, J. and A. T. Peterson. 2005. Interpretation of models of fundamental ecological niches and species' distributional areas. Biodiversity Informatics 2:110.

Svenning, J.-C., C. Fløjgaard, K. A. Marske, D. Nógues-Bravo, and S. Normand. 2011. Applications of species distribution modeling to paleobiology. Quaternary Science Reviews 30:2930-2947.

Szumik, C. and P. Goloboff. 2004. Areas of endemism: an improved optimality criterion. Systematic Biology 53:968-977.

Szumik, C. A., F. Cuezzo, P. A. Goloboff, and A. E. Chalup. 2002. An optimality criterion to determine areas of endemism. Systematic Biology 51:806-816.

Thuiller, W. 2003. BIOMOD - optimizing predictions of species distributions and projecting potential future shifts under global change. Global Change Biology 9:1353-1362.

Thuiller, W., B. Lafourcade, R. Engler, and M. B. Araújo. 2009. BIOMOD - a platform for ensemble forecasting of species distributions. Ecography 32:369-373.

Varela, S., R. P. Anderson, R. García-Valdés, and F. Fernández-González. 2014. Environmental filters reduce the effects of sampling bias and improve predictions of ecological niche models. Ecography:no-no.

Vivo, M. d. 1997. Mammalian evidence of historical ecological change in the Caatinga semiarid vegetation of Northeastern Brazil. Journal of Comparative Biology 2:65-73.

Waltari, E., R. J. Hijmans, A. T. Peterson, A. S. Nyári, S. L. Perkins, and R. P. Guralnick. 2007. Locating Pleistocene Refugia: comparing phylogeographic and Ecological Niche Model predictions. PLoS ONE 2:e563.

Werneck, F. P. 2011. The diversification of eastern South American open vegetation biomes: Historical biogeography and perspectives. Quaternary Science Reviews 30:1630-1648.

Werneck, F. P., G. C. Costa, G. R. Colli, D. E. Prado, and J. W. Sites Jr. 2011. Revisiting the historical distribution of Seasonally Dry Tropical Forests: new insights based on palaeodistribution modelling and palynological evidence. Global Ecology and Biogeography 20:272-288.

Werneck, F. P., C. Nogueira, G. R. Colli, J. W. Sites, and G. C. Costa. 2012. Climatic stability in the Brazilian Cerrado: implications for biogeographical connections 
of South American savannas, species richness and conservation in a biodiversity hotspot. Journal of Biogeography 39:1695-1706.

Willis, E. O. 1992. Zoogeographical origins of eastern Brazilian birds. Ornitologia Neotropical 3:1-15. 
CAPÍtULO 4

Efeitos das mudanças climáticas e fragmentação do habitat sobre a primatofauna do Cerrado 


\title{
RESUMO
}

As atividades antrópicas estão aumentando a concentração de gases do efeito estufa e a temperatura do planeta. As estimativas realizadas do efeito dessa variação do clima na fauna apontam redução da área de distribuição, mudanças no período de reprodução e extinções de algumas espécies no futuro próximo. Somado aos problemas do clima, elevado desmatamento no Cerrado tem ameaçado ainda mais a fauna da região. $\mathrm{O}$ objetivo deste trabalho foi verificar qual será o efeito das mudanças no clima e no uso da terra nos primatas do Cerrado e qual das mudanças terá possivelmente o efeito mais forte. Buscamos ainda projetar mudanças futuras no status de conservação. Projetamos modelos de nicho ecológico com um conjunto de cinco algoritmos, quatro AOGCM (modelo de circulação global atmosfera-oceano) e dois cenários climáticos para os anos de 2050 e 2080. Os modelos foram realizados no pacote biomod2. A mudança no uso da terra foi estimada com o LCM do programa IDRISI para o ano de 2050. Foram utilizados os critérios A e B da IUCN para estimar o grau de ameaça projetado. A diferença na perda de área entre os cenários e condições foi analisada com modelos lineares de efeito misto. Em média, a área projetada das espécies de primatas reduzirá de $9,3 \%$ a 59,2\%, dependendo da capacidade de migração. A área projetada média dentro do Cerrado deve reduzir em até $84,4 \%$ na condição de ocupação somente dos remanescentes nativos. A mudança no uso da terra terá um efeito maior do que o clima sobre a área disponível para primatas. Seis taxa devem aumentar seu nível de ameaça no futuro e seis estão com alto risco de extinção regional no Cerrado. Comparamos com outras estimativas de efeito esperado das mudanças climáticas e discutimos o futuro da espécies de primatas no Cerrado.

\begin{abstract}
Anthropogenic activities are increasing the concentration of greenhouse gases and the planet temperature is consequently also increasing. The estimated effects of this climate variation on the fauna point to a reduction in species' ranges, changes in the reproduction period and extinctions in the near future. In addition to climate changes the Cerrado is suffering a high rate of deforestation, threatening even more the fauna of the region. The objective of this work was to verify what is the effect of climate and land use change in Cerrado primates and which change will possibly have the strongest effect. We also aimed to evaluate future changes in conservation status. Ecological niche models were projected with a set of five algorithms, four AOGCM (atmosphereocean global circulation model) and two climate scenarios to the years 2050 and 2080. The models were done in the biomod 2 package. Land use change was estimated to the year 2050 with LCM in the IDRISI software. On average, the projected area of primate species will reduce $9.3 \%$ to $59.2 \%$, varying according to migration capacity. The mean projected area in the Cerrado shall reduce up to $84.4 \%$ in the condition in which only the native remnants were occupied. Land use change will have a bigger effect than climate in the area available to primates. Six taxa shall increase their threat status in the future and six are at a high risk of regional extinction within the Cerrado. We compare results with other predicted effects of climate change and discuss the future of primate species in the Cerrado.
\end{abstract}




\section{INTRODUÇÃO}

O mundo está passando por um período de aquecimento global pronunciado causado pelo acúmulo de gases causadores do efeito estufa na atmosfera devido às atividades antrópicas (IPCC, 2014). Pelo efeito estufa, a atmosfera terrestre deixa passar a maior parte da radiação solar de ondas curtas, no entanto a radiação de ondas longas (infravermelha) que vem da superfície da Terra é absorvida e irradiada novamente por alguns gases, como $\mathrm{CO}_{2}, \mathrm{CH}_{4}$ e $\mathrm{N}_{2} \mathrm{O}$, tornando a superfície do planeta mais quente (Chapin et al. 2002). A exacerbação desse fenômeno está acarretando a elevação da temperatura global e os efeitos desse aquecimento já podem ser percebidos nas populações naturais de animais, com a diminuição da área ocupada por espécies e mudanças na época de reprodução (Root et al. 2003, Araújo et al. 2005).

Mudanças climáticas cíclicas já ocorrem no planeta naturalmente há milhares de anos (Salgado-Labouriau 1994, Petit et al. 1999). Entre cada um dos períodos de era glacial a temperatura do planeta passou por momentos de intervalos mais quentes, como o que estamos vivendo atualmente. No entanto, o painel intergovernamental para mudanças climáticas (IPCC) (IPCC 2007) estima que nos últimos 250 anos a temperatura do planeta passou a aumentar muito mais rapidamente do que o padrão existente nos últimos 10.000 anos devido às emissões de gases do efeito estufa por atividades humanas. O IPCC5 trabalhou as análises do clima da Terra no futuro baseado em quatro cenários que prevêem diferentes condições econômicas e sociais nos próximos anos e de acordo com o cenário escolhido a temperatura deve aumentar de 1.1 a $4.8^{\circ} \mathrm{C}$ até 2100 (IPCC 2014).

Além do cenário de mudanças climáticas globais, o Cerrado também sofre com alterações e fragmentação do habitat de forma acelerada. Mais da metade da cobertura vegetal original do Cerrado já foi convertida para usos antrópicos e se o processo de 
fragmentação continuar nesse ritmo até o ano de 2030 não haverá mais área natural restante no bioma fora das áreas protegidas (Machado et al. 2004, Klink and Machado 2005). Um estudo recente calculou a projeção de desmatamento para 2050 e estimou que nesse ano quase toda a área nativa do sul e centro do Cerrado já terá sido desmatada (Faleiro et al. 2013).

O crescente aumento da antropização do domínio nos últimos anos tem aumentado consideravelmente a ameaça para a biodiversidade da região, em especial para espécies dependentes de ambientes arbóreos, como os primatas. Dos primatas que possuem parte da sua distribuição geográfica dentro do bioma Cerrado, cinco estão ameaçadas de extinção pela lista de mamíferos ameaçados oficial do Brasil (MMA 2014) e três estão ameaçadas de acordo com a lista vermelha da IUCN (IUCN 2012). A principal ameaça para os primatas ameaçados no Cerrado é a perda de área por desmatamento, a conversão de áreas nativas em áreas agrícolas e a caça (Machado et al. 2008a). Quatro espécies entraram na lista de ameaçados por causa da significativa redução no tamanho populacional nos últimos anos (critério A) (MMA 2014). De acordo com a IUCN, 36,4\% dos primatas brasileiros estão ameaçados de extinção, o que significa que a porcentagem de ameaçados para o Cerrado $(18,7 \%)$ é menor do que a média para o Brasil. Como a situação atual de conservação dos primatas do Cerrado ainda está melhor que a média nacional, é possível que exista mais tempo para se planejar uma estratégia de conservação para o grupo antes que a situação piore.

As espécies de primatas brasileiros são dependentes de ambientes florestais, mesmo que consigam ocorrer temporariamente em alguns ambientes antropizados (Estrada 2006), pois dependem da a existência de um estrato arbóreo. A tendência atual de diminuição dos ambientes florestais no Brasil pode causar um impacto sério nas espécies de primatas existentes. Tendo uma estimativa da resposta das espécies às 
mudanças ambientais e antrópicas podemos nos antecipar para a conservação das espécies antes do efeito ocorrer, resultando em propostas de manejo mais eficientes.

Uma ferramenta poderosa para estimar os efeitos das mudanças ambientais do globo nas espécies de primatas é a modelagem de nicho ecológico (ENM) (Elith and Leathwick 2009b, Peterson et al. 2011). Esta ferramenta utiliza algoritmos estatísticos para fazer um modelo preditivo de ocorrência potencial da espécie usando as variáveis climáticas e ambientais existentes em cada ponto de ocorrência (Soberón and Peterson 2005, Peterson et al. 2011). As variáveis climáticas utilizadas podem ser do tipo condição/ grineliana, quando não interagem com a espécie ou são características intrínsecas do meio; ou variável recurso/ eltoniana, quando são consumidas ou referentes à interação entre espécies (Soberón 2007, Polechová and Storch 2008). Normalmente se utiliza as variáveis do tipo condição para conseguir descrever processos em uma escala continental (Soberón 2007) e para projetar para outros períodos de tempo (Peterson et al. 2011), pois essas variáveis são mais fáceis de estimar e a hipótese do ruído Eltoniano postula que variáveis recurso são só ruído, pois os modelos são precisos mesmo sem elas (Soberón and Nakamura, 2009). O método assume que as espécies irão ocupar o mesmo nicho ambiental nos períodos de tempo considerados porque as espécies tendem a manter os seus requisitos de nicho ao longo de muito tempo, conceito conhecido como conservatismo de nicho (Peterson et al. 1999). O ENM foi usado para estimar a perda de área de espécies causada por mudanças climáticas em vários grupos (Bakkenes et al. 2002, Thomas et al. 2004, McClean et al. 2006, Sekercioglu et al. 2008, Barbet-Massin et al. 2012, Russell et al. 2012).

Neste capítulo buscamos identificar como as mudanças climáticas globais e as mudanças no uso da terra afetarão as populações de primatas no Cerrado. Buscamos verificar: qual fator terá maior impacto no conjunto de primatas, se fragmentação do 
habitat ou mudanças climáticas e se o status de conservação dos primatas irá se modificar nos próximos anos. Nossas hipóteses foram: as mudanças no uso da terra terão um impacto maior que as mudanças climáticas na distribuição de primatas do Cerrado e os primatas apresentarão um grau de ameaça maior no futuro.

\section{MATERIAL E MÉTODOS}

\section{Modelagem de Nicho Ecológico}

Para a construção do conjunto dos modelos de nicho foi utilizada a mesma base de dados gerada para o capítulo 2 (esse volume), composta por dados de ocorrência de museus e coleções online, registros de especialistas obtidos pelo Centro de Primatas Brasileiros do ICMBIO e ocorrências relatadas na literatura científica.

Foi utilizado um conjunto de variáveis ambientais que envolvem precipitação e temperatura no presente e projetadas para as mesmas variáveis nos anos de 2050 e 2080 . Foram obtidas as variáveis da base de dados online Worldclim (Hijmans et al. 2005; www.worldclim.org) que contém variáveis com diferentes relações de temperatura ou precipitação globais ao longo do ano. Foram utilizadas as variáveis bioclimáticas bio 1 a bio 7 e bio 10 a bio 17 , porque as variáveis bio 8, 9, 18 e 19 continham artefatos para a região de estudo. Novas variáveis foram criadas com o uso de análise de componentes principais (PCA), para resumir a variação dos dados em menos dimensões. A redução do número de variáveis pode diminuir o overfitting (ajuste exagerado às variáveis) que tende a ser maior com maior número de dimensões. Foram utilizados somente os sete primeiro eixos da PCA que explicavam juntos $99 \%$ da variação dos dados.

As projeções para o futuro foram baseadas em estimativas de modelos de circulação geral atmosfera-oceano (AOGCM) utilizados no quinto painel 
intergovernamental sobre mudanças climáticas (IPCC) (IPCC 2014). Foram utilizados quatro modelos AOGCM de diferentes fontes: da universidade de Miami RSMASCCSM4; do Centro Canadense para modelagem e análise CCMA-CANESM2; da organização para comunidade científica e pesquisa industrial da Austrália CSIRO-MK3 e do Met Office Hadley Centre, com contribuição do INPE, MOHC-HADGEM2. Para cada AOGCM foram utilizados os representative concentration pathways (RCP) RCP2.6 e RCP8.5. RCPs são cenários climáticos utilizados no quinto relatório do IPCC que descrevem concentrações prováveis dos gases de efeito estufa na atmosfera e a forçante radiativa no ano de 2100 (IPCC 2014). O RCP2.6 é o cenário mais otimista considerando que as emissões de gases do efeito estufa atingirão um pico em 2020 e depois declinarão e o RCP8.5 é o mais pessimista, pois considera que as emissões continuam a aumentar indefinidamente.

Para realizar as análises de modelagem de nicho ecológico foram testados nove modelos, de forma a fazer uma previsão usando um conjunto de modelos disponíveis no pacote Biomod2 (Thuiller 2003; Thuiller et al. 2009). É preferível usar o consenso de um conjunto de modelos a usar apenas um, pois o consenso gera um resultado mais preciso por reduzir as incertezas específicas de cada modelo (Araújo et al. 2005; Marmion et al. 2009). Foi feita uma primeira análise com todos os modelos, e selecionamos apenas aqueles cinco modelos que apresentaram maior AUC (método que estima a precisão do modelo a partir da adequação à curva ROC) para os nossos dados, segundo método proposto recentemente (Qiao et al. 2015). Foram selecionados e utilizados nos modelos finais os seguintes algoritmos: (1) modelo aditivo generalizado (GAM); (2) modelo impulsionado generalizado (GBM); (3) redes neurais artificiais (ANN); (4) Máxima entropia (Maxent) (Philips et. al. 2004) e (5) floresta randômica 
(RF). Detalhes dos métodos de cada algoritmo podem ser encontrados em revisões específicas (Hastie et al. 2009, Peterson et al. 2011).

Mesmo sem ter dados confiáveis de ausência das espécies é vantajoso utilizar pseudo-ausências porque as predições de modelos que somente utilizam presenças são menos precisas (Elith et al. 2006). Utilizamos um algoritmo para gerar pseudoausências que utilizou pontos que caem fora de uma modelagem ecológica de somente presença, chamada de envelope de superfície de distribuição (SRE). Esse método está disponível no Biomod2 e outros estudos já o utilizaram com bons resultados (Marini, et al. 2010; Le Maitre et al. 2008). Somente os modelos com AUC/ROC maior que 0,80 foram selecionados para produzir um mapa de consenso. Foi utilizada a média do valor de cada pixel dos modelos para gerar a adequabilidade climática da espécie em um dado lugar. Para transformar os resultados dos modelos em mapas de ocorrência potencial foram cortados os valores de adequabilidade ambiental de cada pixel seguindo limiar que maximiza o valor da True Skill Statistics (TSS) (Allouche et al. 2006). Todos os mapas e imagens utilizados foram reamostrados para a resolução de 2,5 arcos de minuto.

\section{Grau de ameaça de extinção no futuro}

Foi utilizada uma projeção da área de remanescente nativa do Cerrado calculada para o ano de 2050 através das imagens de remanescentes vegetais nativos de 2002 e 2008 divulgadas pelo centro de sensoriamento remoto do IBAMA (http://siscom.ibama.gov.br/monitorabiomas/index.htm). O uso da terra foi modelado com o Land Change Modeler - LCM do software Idrisi (Eastman 2009), usando as variáveis: elevação do terreno, precipitação anual, proximidade de estradas, proximidade de cidades e proximidade de áreas desmatadas. O LCM é um 
procedimento de aprendizado e classificação automática que faz a projeção para o futuro utilizando cadeias de Markov. Para testar a precisão da previsão invertemos a análise e projetamos o mapa para 1990 e fizemos uma inspeção visual em imagens MrSID de 1990 (https://zulu.ssc.nasa.gov/mrsid/).

Essa análise foi considerada como a estimativa do desmatamento que ocorrerá no Cerrado caso o ritmo permaneça igual ao presente nos cálculos da cobertura vegetal atual. Como todos os primatas neotropicais necessitam em algum grau de ambientes arbóreos para viver, retiramos as áreas que foram previstas como antropizadas da área projetada para ocorrência das espécies no futuro.

Também foram consideradas duas condições para o deslocamento das espécies, uma em que elas podem se deslocar livremente e outra em que elas não poderão ocupar áreas que elas não ocupam no presente. O motivo de restringir a possibilidade de migração é que a movimentação por áreas inadequadas na paisagem e ocupação de uma nova área distante é algo difícil de ocorrer, pois primatas têm dificuldade em se movimentar por ambientes sem árvores ou deslocar pela matriz na paisagem (Vasudev and Fletcher 2015).

Analisamos quanto cada espécie potencialmente perderá ou ganhará em área ocupada no Cerrado e as mudanças na adequabilidade do ambiente para cada espécie de forma a estimar o risco de extinção de cada espécie no futuro. Foram utilizados os critérios A e B da IUCN (IUCN 2001) para estimar o risco de extinção das espécies em análise. O critério A trata da redução do tamanho da população e para estimar essa redução foi considerado que a densidade populacional permanece igual ao longo do tempo e, portanto, a redução na área é proporcional à redução na população. O critério B trata de distribuição geográfica restrita. Para o critério A3 foi considerado que a espécie perdeu a cada dez anos $30 \%$ da população para vulnerável, $50 \%$ para em perigo 
ou $80 \%$ para criticamente em perigo (IUCN 2001), o que equivale a, respectivamente, $65,7,87,5$ e $99,2 \%$ ao final de 30 anos. Se a projeção da espécie estimou um ganho de área foi considerada como de menor preocupação (LC). Para o critério B a espécie teria que ter, em área potencial, menos de $20.000 \mathrm{~km}^{2}$ para ser considerada vulnerável, menos de $5.000 \mathrm{~km}^{2}$ para ser considerada em perigo e menos de $100 \mathrm{~km}^{2}$ para atingir o status de criticamente em perigo (IUCN 2001). Para o cálculo das áreas os arquivos foram reprojetados para a projeção cônica Albers equal-area com células de 4,8 x 4,8 km, porque essa projeção não apresenta distorções para o tamanho das feições mapeadas.

\section{$\underline{\text { Análises estatísticas }}$}

As diferenças entre as áreas nas projeções foram analisadas com dois modelos de efeito misto, um para área total disponível e outro só considerando a área no Cerrado. Cada modelo comparou o logaritmo das áreas $\left(\mathrm{em}^{\mathrm{km}}{ }^{2}\right)$ com as variáveis cenário climático e restrição de ocupação de efeito fixo e a variável espécies de efeito randômico. As áreas foram transformadas com logaritmo natural para obedecer ao requisito de normalidade do teste. A variável cenários possui os fatores presente, otimista 2050 (O.50), otimista 2080 (O.80), negativo 2050 (N.50) e negativo 2080 (N.80) e a variável condição possui os fatores migração ilimitada (MI), sem migração (SM) e do uso da terra (UT) (Figura 1). Os testes foram conduzidos utilizando os pacotes raster (Hijmans 2015), nlme (Pinheiro et al. 2015), lme4 (Bates et al. 2014) e lsmeans (Lenth and Hervã 2015) do software R 3.1.2 (R core team 2014). 

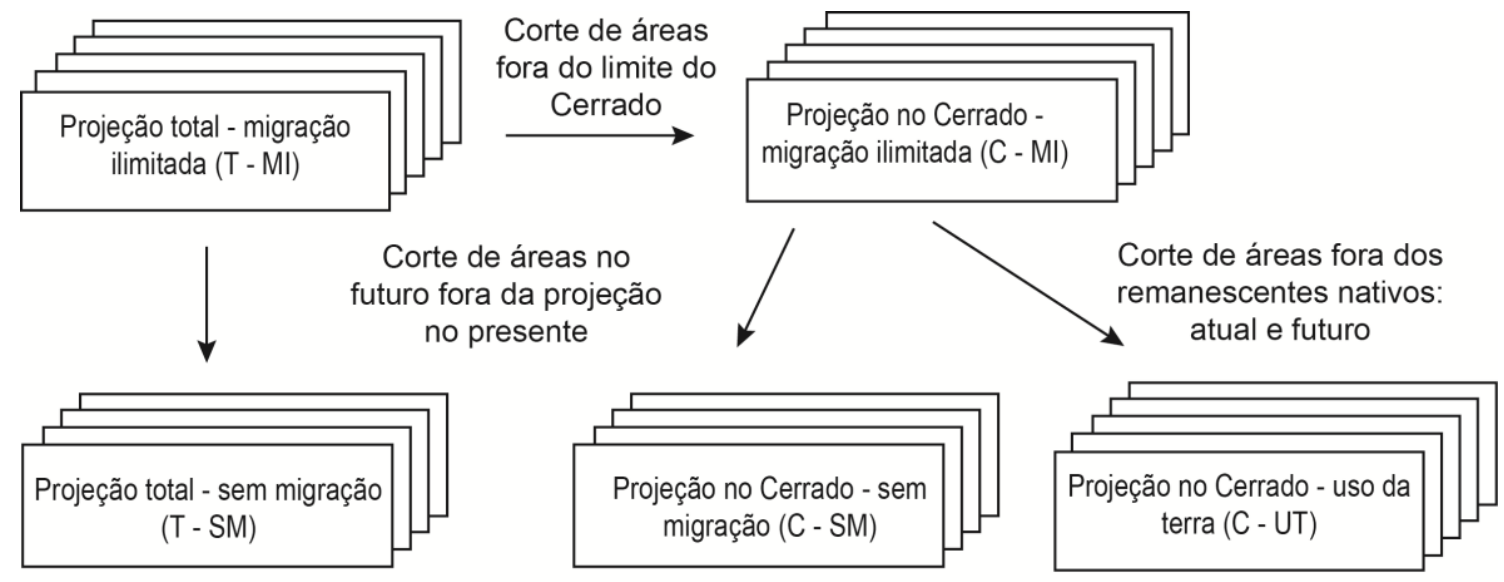

Figura 1. Diagrama explicativo de como foram gerados os fatores de condição de deslocamento possíveis para as espécies.

\section{RESULTADOS}

Os modelos gerados tiveram alta capacidade preditiva, com um valor de AUC médio em 0,99 (desvio padrão $=0,01)$ e de TSS médio em 0,96 (desvio-padrão $=0,02)$ e apresentaram sensitividade média em 97,51 e especificidade em 98,32. Os mapas indicativos das variações na adequabilidade climática média de todas as espécies nos anos de 2050 e 2080 tanto nos cenários positivos quanto negativos (Figura 2) mostram um empobrecimento geral do habitat no futuro, representado pela diminuição dos valores de adequabilidade. Enquanto o padrão da distribuição das espécies no cenário otimista parece ser uma versão do presente um pouco mais fraco, no cenário pessimista o padrão se mostra diferente (Figura 2). 


\section{Presente}

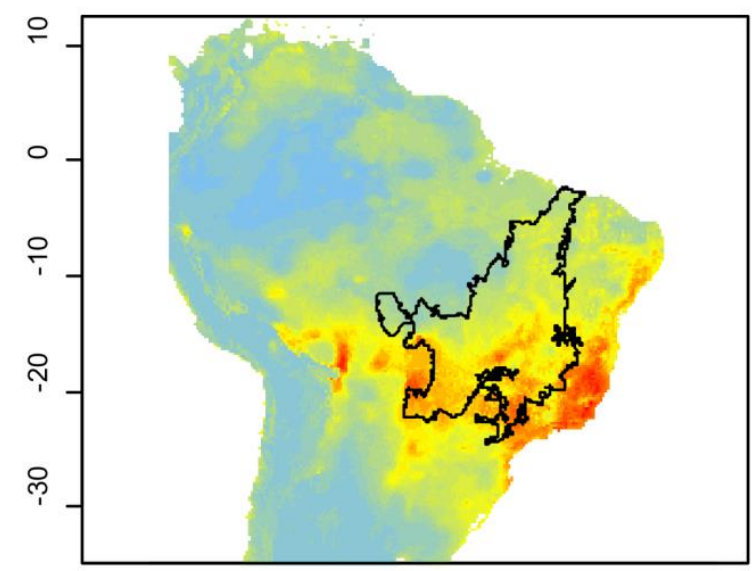

RCP 2.6 - ano 2050

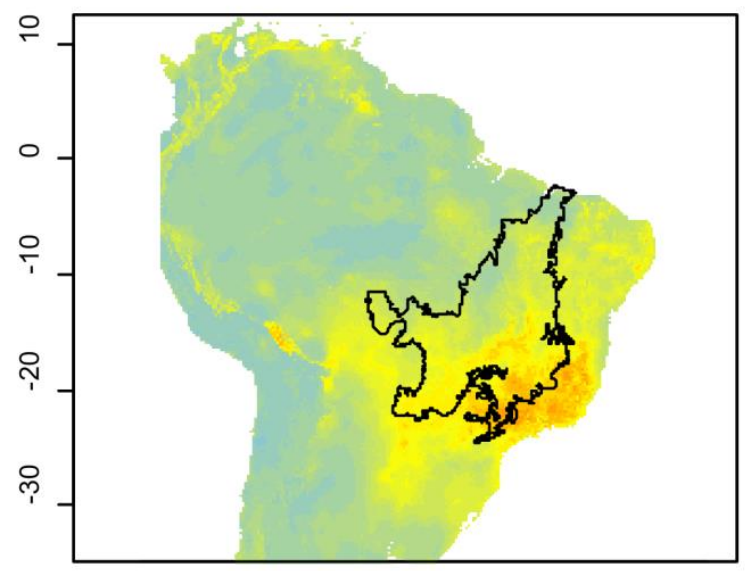

RCP 8.5 - ano 2050

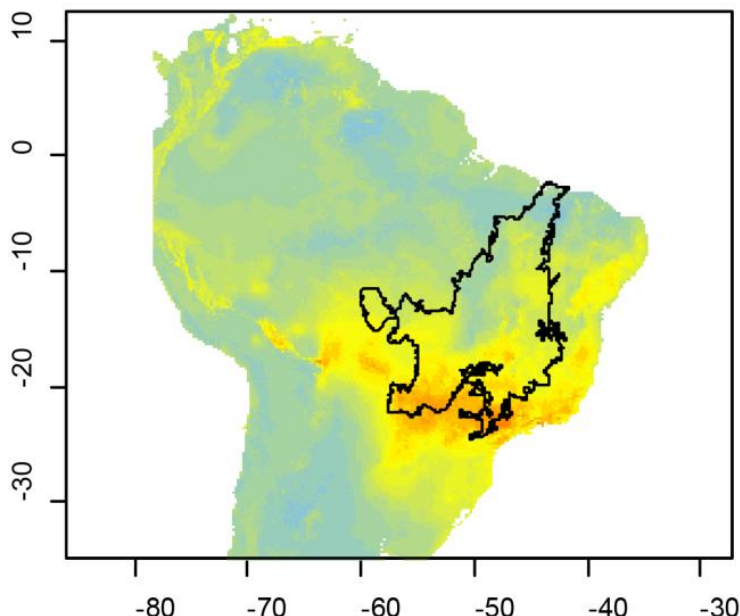

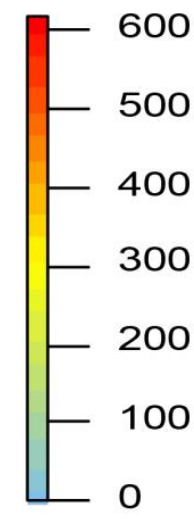

RCP 2.6 - ano 2080

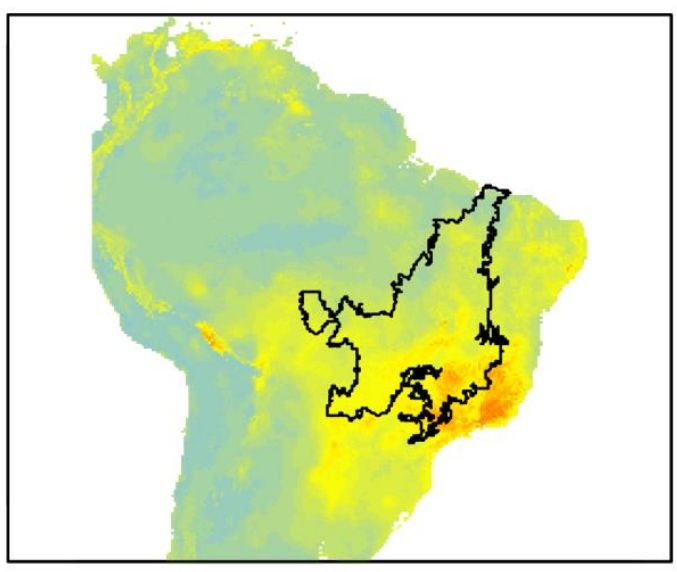

RCP 8.5 - ano 2080

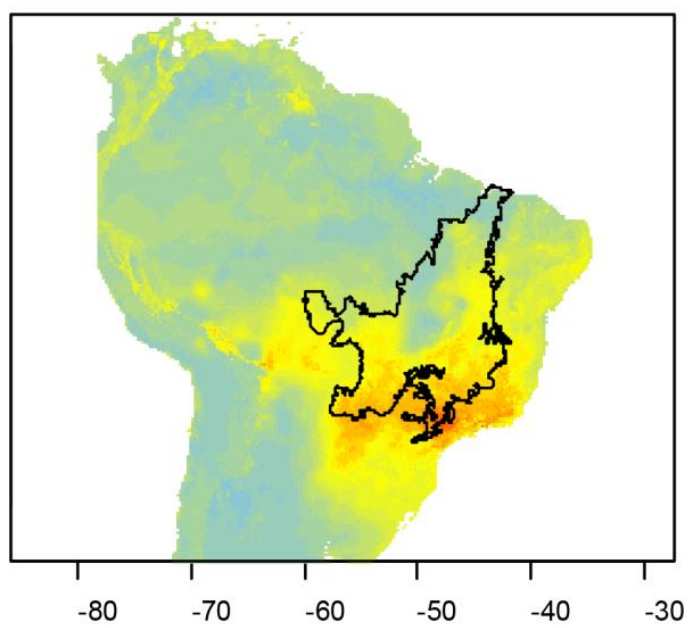

Figura 2. Figura representando a adequabilidade climática média (cor) para os primatas do Cerrado no presente (esquerda, no alto) e nos anos de 2050 (esquerda) e 2080 (direita) para os cenários otimista (RCP 26 no centro) e pessimista (RCP 85 em baixo). 
A riqueza potencial de espécies caiu em todas as áreas quando se restringiu a dispersão, com algumas áreas perdendo até dez espécies em relação ao que têm no presente (Figura 3). As principais áreas com perda de riqueza de espécies no Cerrado se encontram no Planalto Central e no Mato Grosso. Já na região fora do Cerrado foram observadas perdas expressivas no Pantanal e no litoral do sudeste do Brasil. Áreas com baixa riqueza atual tiveram um alto percentual de perda de espécies, apesar do baixo valor absoluto mostrado, como é o caso das áreas mais ao norte do Cerrado.

A área projetada total irá reduzir independentemente do cenário para 43,7\% dos táxons e apenas 12,5\% irão aumentar a área na condição de deslocamento ilimitado. Em média, a área projetada total das espécies de primatas do Cerrado irá reduzir em 9,3\% na condição de deslocamento ilimitado e 59,2\% para a condição sem migração (tabela 1). Considerando somente a área dentro do Cerrado, $56,2 \%$ das espécies irão reduzir de área e $12,5 \%$ irão ampliar a área de ocorrência dentro do domínio. Além disso, os táxons deverão, em média, aumentar a área em 2,7\% com deslocamento ilimitado, reduzir em 59,0\% sem migração e reduzir em $84,4 \%$ na condição de ocupação somente da área remanescente no Cerrado. 


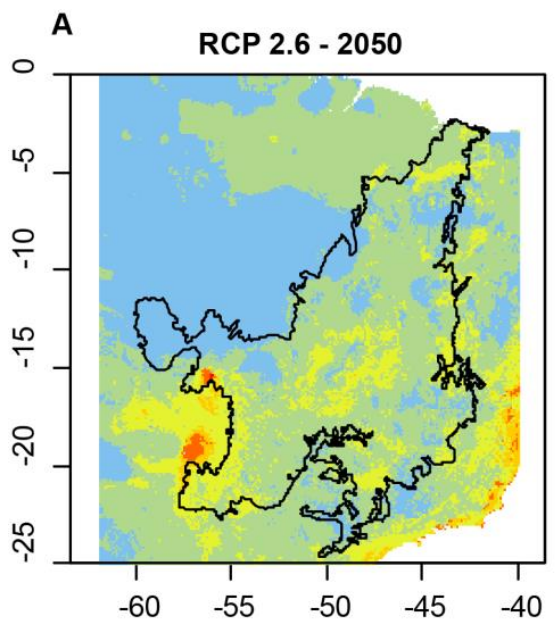

B
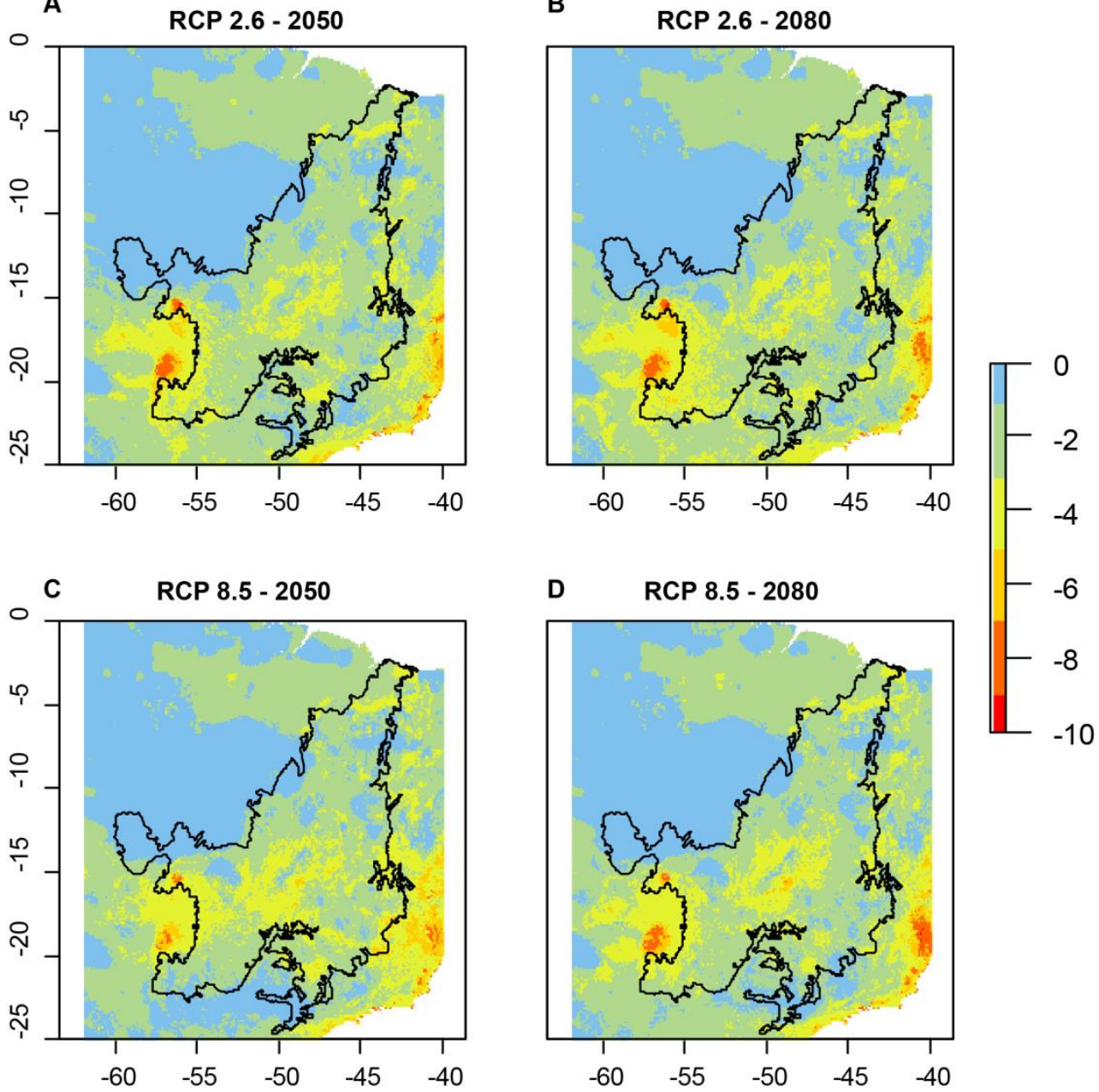

Figura 3. Mudança na riqueza de espécies ('turnover') existente entre o presente e o ano de 2050 (A e C) e o ano de 2080 (B e D), nos cenários otimista (A e B) e pessimista (C e D). Azul representa que a área não perderá riqueza no futuro, enquanto amarelo e vermelho representa a quantidade de espécies que cada célula perderá. 
Tabela 1. Valores estimados dos percentuais de perdas de área projetadas para as espécies do Cerrado nos anos de 2050 e 2080 e nos cenários otimista e pessimista. Foi considerado que os táxons não terão liberdade para migrar para locais em que eles não estavam no presente.

\begin{tabular}{|c|c|c|c|c|c|c|c|c|}
\hline & \multicolumn{4}{|c|}{ Área total } & \multicolumn{4}{|c|}{ Cerrado } \\
\hline & \multicolumn{2}{|c|}{ RCP2.6 } & \multicolumn{2}{|c|}{ RCP8.5 } & \multicolumn{2}{|c|}{ RCP2.6 } & \multicolumn{2}{|c|}{ RCP8.5 } \\
\hline & 2050 & 2080 & 2050 & 2080 & 2050 & 2080 & 2050 & 2080 \\
\hline Alouatta belzebul & 82,54 & 82,31 & 61,24 & 86,07 & 100,00 & 100,00 & 100,00 & 100,00 \\
\hline Alouatta caraya & 13,88 & 13,73 & 14,31 & 18,62 & 9,72 & 4,64 & 14,31 & 15,62 \\
\hline Alouatta guariba & 61,00 & 60,62 & 45,73 & 45,29 & 25,92 & 38,65 & 12,60 & 4,36 \\
\hline Aotus a. azarae & 95,74 & 96,29 & 90,21 & 97,26 & 62,64 & 67,64 & 48,57 & 30,19 \\
\hline Aotus a. infulatus & 76,84 & 72,29 & 65,85 & 59,39 & 99,94 & 99,92 & 100,00 & 100,00 \\
\hline $\begin{array}{l}\text { Callicebus } \\
\text { donacophilus }\end{array}$ & 98,16 & 95,48 & 76,34 & 75,40 & 99,98 & 98,25 & 89,63 & 62,47 \\
\hline $\begin{array}{l}\text { Callicebus } \\
\text { nigrifrons }\end{array}$ & 30,15 & 31,67 & 26,63 & 26,22 & 44,71 & 42,35 & 29,74 & 33,35 \\
\hline $\begin{array}{l}\text { Callicebus } \\
\text { personatus }\end{array}$ & 40,26 & 48,39 & 48,32 & 49,03 & 19,01 & 38,27 & 35,42 & 34,89 \\
\hline $\begin{array}{l}\text { Callithrix } \\
\text { geoffroyi }\end{array}$ & 87,25 & 90,00 & 68,01 & 68,44 & 89,77 & 93,38 & 62,27 & 51,55 \\
\hline $\begin{array}{l}\text { Callithrix } \\
\text { jacchus }\end{array}$ & 50,83 & 50,33 & 68,42 & 65,90 & 97,23 & 98,24 & 99,85 & 99,75 \\
\hline $\begin{array}{l}\text { Callithrix } \\
\text { penicillata }\end{array}$ & 30,48 & 33,44 & 26,37 & 25,68 & 26,56 & 31,64 & 16,54 & 20,29 \\
\hline Mico melanurus & 98,09 & 99,21 & 100,00 & 99,99 & 97,76 & 98,44 & 100,00 & 100,00 \\
\hline Sapajus cay & 20,23 & 21,93 & 38,79 & 32,16 & 1,93 & 3,70 & 42,27 & 20,93 \\
\hline $\begin{array}{l}\text { Sapajus } \\
\text { libidinosus }\end{array}$ & 95,60 & 96,16 & 92,91 & 95,90 & 95,82 & 96,66 & 93,03 & 96,38 \\
\hline Sapajus nigritus & 29,09 & 28,93 & 30,71 & 34,23 & 2,34 & 0,0 & 2,47 & 4,39 \\
\hline $\begin{array}{l}\text { Sapajus } \\
\text { xanthosternos }\end{array}$ & 56,27 & 66,46 & 49,73 & 52,41 & 100,00 & 100,00 & 90,91 & 78,41 \\
\hline
\end{tabular}


Os cenários de mudança do clima não afetaram significativamente a área dos primatas analisados em relação ao presente para a área total de cada espécie $\left(\mathrm{F}_{4,123}=0,38 ; \mathrm{p}=0,82\right)$. Em relação à área ocupada no Cerrado, os cenários de clima reduziram a área significativamente $\left(\mathrm{F}_{4,202}=12,29 ; \mathrm{p}<0,001\right)$, existindo diferença de área entre todos os cenários do futuro e do presente (P x P.50: $t_{202}=5,47 ; \mathrm{p}<0,001 ; \mathrm{P} \times \mathrm{P} .80$ : $\mathrm{t}_{202}=5,54 ; \mathrm{p}<0,001 ; \mathrm{P} \times \mathrm{N} .50: \mathrm{t}_{202}=6,62 ; \mathrm{p}<0,001 ; \mathrm{P} \times \mathrm{N} .80: \mathrm{t}_{202}=5,44 ; \mathrm{p}<0,001$ ), porém não entre os cenários do futuro ( $p>0,05)$. Já as condições de deslocamento e uso da terra provocaram uma diminuição significativa da área dos táxons. Quando se considera a área toda obtivemos uma área muito maior para deslocamento ilimitado do que quando as projeções eram restritas na possibilidade de migração $\left(F_{1,123}=48,28 ; p<0,001\right)$ (Figura 4). $\mathrm{Na}$ área no Cerrado também houve uma variação significativa nas áreas entre as condições $\left(\mathrm{F}_{2,202}=15,58 ; \mathrm{p}<0,001\right)$, no entanto o fator que mais afetou foi o uso da terra (DI x UT: $\mathrm{t}_{202}=5,38 ; \mathrm{p}<0,001 ; \mathrm{SM}$ x UT: $\mathrm{t}_{202}=2,75 ; \mathrm{p}=0,02$ ) e não a possibilidade de migração (DI x SM: $\left.t_{202}=2,18 ; \mathrm{p}=0,08\right)$ (Figura 4). 

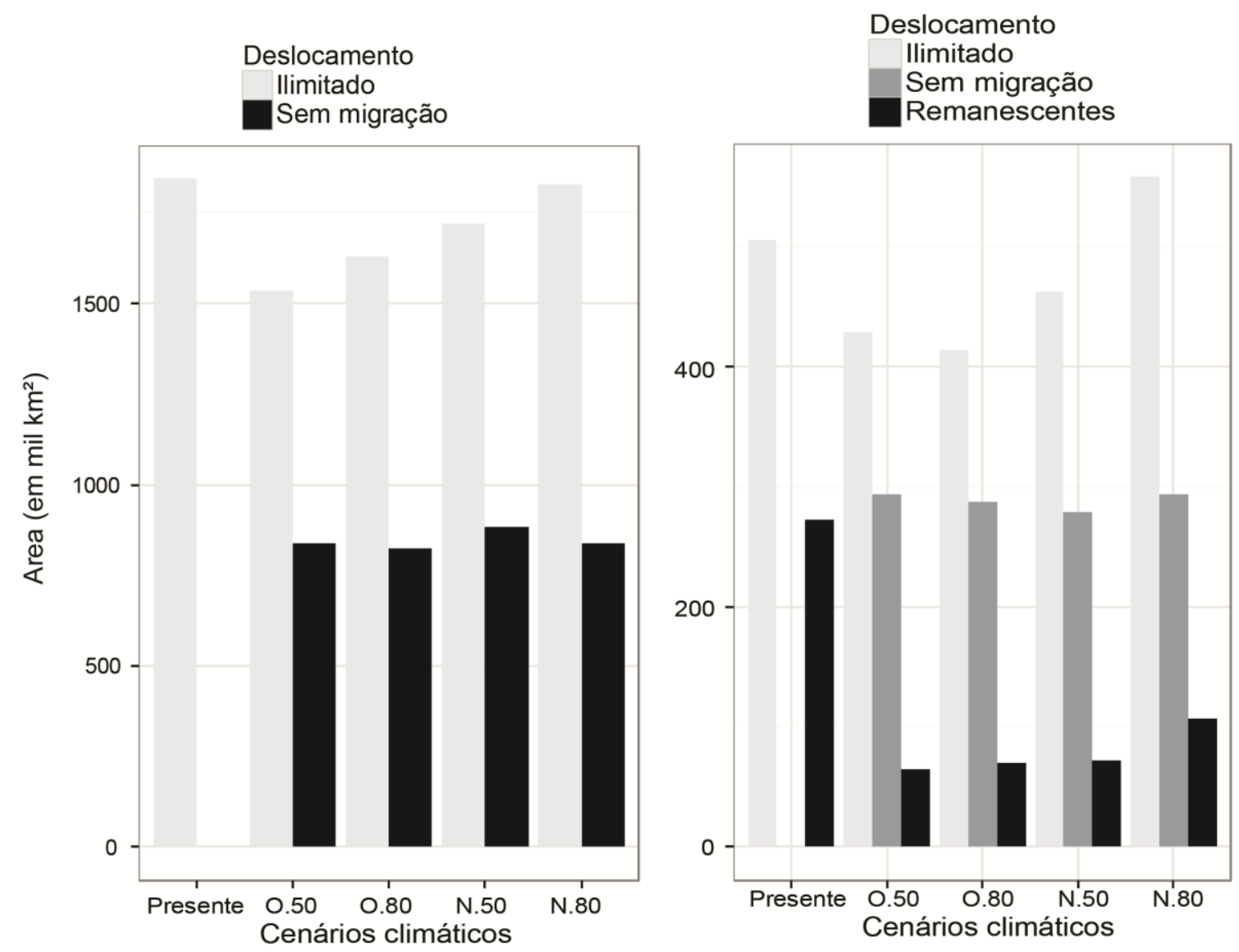

Figura 4. Representação gráfica das diferenças de áreas projetadas nos diferentes cenários climáticos (presente, otimista 2050, otimista 2080, negativo 2050 e negativo 2080) e nas diferentes condições de deslocamento. Na parte da esquerda, gráfico com a área em todo o mapa e na parte da direita somente no Cerrado.

As adequabilidades climáticas para as espécies se deslocarão de forma variada.

Espécies que possuem área na Amazônia terão deslocamento para o oeste. As que possuem área na região do Chaco deverão perder adequabilidade e se deslocarão para o sul ou para o norte. Cinco espécies ganharão adequabilidade para dentro da região do Cerrado. O padrão médio de deslocamento das espécies do presente para uma média dos cenários climáticos utilizados (média mostrada porque não houve diferença entre a projeção dos cenários) está mostrado na figura 5. 

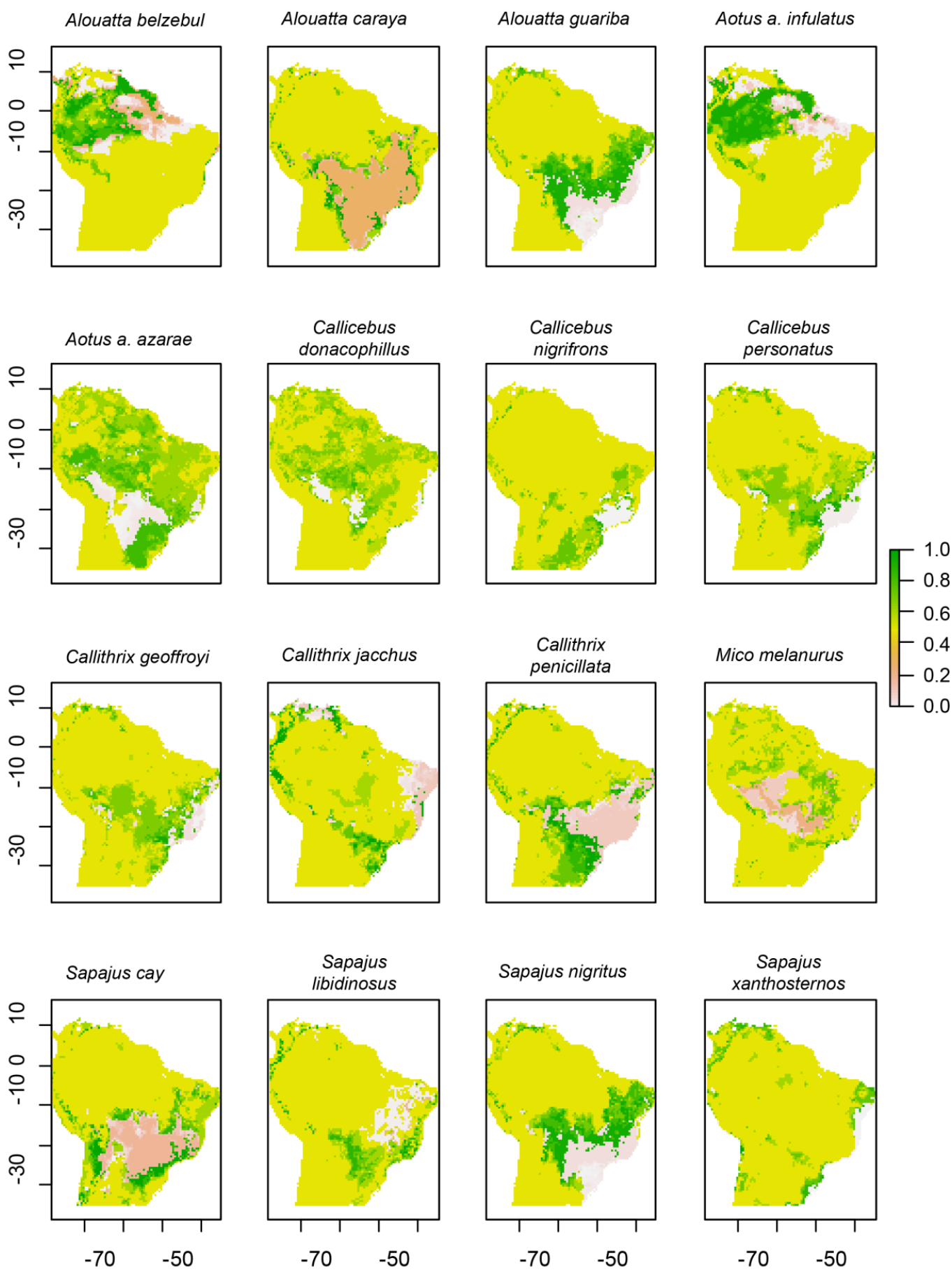

Figura 5. Análises pareadas mostrando diferenças significativas entre os pares de projeções presente e negativo 2050 para cada espécie. Cores representam com qual cenário a célula está mais semelhante: quanto mais próximo do verde mais parecido somente com o futuro e mais próximo do branco significa só semelhante ao presente. Verde escuro representa diferença significativa do presente, áreas em branco são diferentes do futuro, áreas em rosa não demonstram diferença entre presente e futuro e áreas em bege não são ocupadas. 
Para o ano de 2050, o Cerrado deve ficar muito deficiente em sua primatofauna, pois as projeções apontam que seis táxons terão uma redução de área maior que $99 \%$ em pelo menos dois cenários do futuro. Destas, Alouatta belzebul, Aotus a. infulatus, Mico melanurus e Sapajus xanthosternos estão com uma previsão de $100 \%$ de redução, ou seja, previsão de extinção na região (Tabela 2). Essa é a situação considerando a projeção de mudanças no uso da terra. Para a condição só no Cerrado e sem restrições de deslocamento, sete espécies devem aumentar o seu grau de ameaça regional e três podem passar para criticamente em perigo na região (Tabela 2). Nessa condição mencionada, a espécie Alouatta guariba poderá aumentar de área no Cerrado. Considerando toda a área das espécies e sem possibilidade de migração, espera-se que seis espécies devam aumentar o risco de extinção, Alouatta guariba deve sair da lista de ameaçados e Mico melanurus pode ficar criticamente em perigo (Tabela 2). 
Tabela 2. Graus de ameaça de extinção no presente e no ano de 2050 para as condições sem migração, somente no Cerrado e de desmatamento no Cerrado. As letras correspondem aos níveis de ameaça: menor preocupação (LC), quase ameaçado (NT), vulnerável (VU), em perigo (EN) e criticamente em perigo (CR). Espécies em negrito possuem risco crítico em mais de uma condição.

\begin{tabular}{llllllll}
\hline & Presente & Sem migração & & Cerrado* & Desmatamento no \\
Cerrado*
\end{tabular}

\footnotetext{
*Todas as estimativas dessas colunas são para ameaças de extinção regionais, somente no Cerrado
} 


\section{DISCUSSÃO}

\section{Cenários climáticos}

Os cenários de mudança climática global reduziram a área que os táxons ocuparam no Cerrado, mas não afetaram consideravelmente a área ocupada na abordagem distribuição total. Isso significa que a maioria das espécies foi projetada em maior proporção em áreas fora do domínio do Cerrado. Várias espécies podem mudar de área procurando áreas mais frias, como o sul do continente ou o alto das montanhas, outras aumentaram a sua distribuição dentro do Cerrado, o que mostra que cada uma respondeu de forma diferente aos efeitos do clima.

Dentro dos cenários globais de alterações climáticas, muitos estudos já projetaram mudanças na distribuição de espécies para o futuro (Bellard et al. 2012). Na Europa, cada local perderá em média $32 \%$ da sua diversidade de plantas até 2050 e a área total em que serão perdidas $32 \%$ das espécies ou mais ocupam $44 \%$ do continente (Bakkenes et al. 2002). Outro estudo estimou em aproximadamente 30\% a perda de espécies (dependendo do cenário) e 50\% a mudança na composição de espécies de plantas (Thuiller 2005). As espécies de plantas se moverão em direção ao norte do continente, que é mais frio (Bakkenes et al. 2002). As espécies de peixes do Mediterrâneo também mudarão sua distribuição em direção ao norte, sendo que em média 37,85 \% dos peixes do mar Mediterrâneo migrarão para o norte em torno de 70 km até 2069 e $53.82 \%$ das espécies migrarão em torno de 90 km para o norte até 2099 (Albouy et al. 2012). Aves europeias terão uma distribuição de 50 a $80 \%$ do tamanho da distribuição atual e a área de $71 \%$ das espécies irá reduzir, com apenas $40 \%$ de sobreposição com o presente, variando para o nordeste (Huntley et al. 2006, BarbetMassin et al. 2012). Na África, é esperada que 54 a $74 \%$ da biodiversidade de 
vertebrados reduza de área (Garcia et al. 2012). Aves deverão perder de 35 a $45 \%$ para de área nas áreas de importância (IBA) (Hole et al. 2009) e mamíferos deverão perder em torno de 30 a 50\% de área (Thuiller et al. 2006). Plantas da África se moverão para altitudes maiores e 81-97\% das espécies reduzirão de área (McClean et al. 2006). O Cerrado brasileiro terá redução de área para 25 de 26 espécies endêmicas de aves de ampla distribuição, com uma mudança da área de distribuição de 175 a 200 km na direção sudeste (Marini et al. 2009). No presente estudo, de 44 a $87 \%$ das espécies analisadas deverão reduzir de área dependendo do cenário considerado e as espécies reduzirão sua área em média 59\%.

Espera-se que os biomas da América do Sul também variem conforme a mudança do clima, com as áreas de savana aumentando de proporção no continente, forçando a perda da parte leste da floresta Amazônica e aparecimento de novas áreas de florestas no sul do continente (Salazar et al. 2007). Essa velocidade de mudança das distribuições das formações vegetais é questionável, já que 80-100 anos pode ser pouco tempo para o crescimento completo de uma nova vegetação. No entanto, a velocidade das mudanças climáticas na região também é muito maior do que no passado (em torno de $10 \mathrm{~m} /$ ano nos últimos $21 \mathrm{mil}$ anos e $1 \mathrm{~km} /$ ano nos próximos 100 anos (Loarie et al. 2009, Sandel et al. 2011). Essa velocidade gera uma pressão seletiva que seleciona as espécies que conseguirem se adaptar fisiologicamente,fenologicamente ou comportamentalmente ou migrar para uma nova distribuição (Root et al. 2003, Parmesan 2006, Bellard et al. 2012), caso contrário ocorrerá a extinção dessas espécies Se as espécies de primatas do Cerrado puderem migrar livremente elas terão respostas acompanhando a mudança esperada para os biomas, algumas acompanhando formações florestais e outras se expandirão junto com o Cerrado. 
Os cenários de mudanças climáticas adotados são os mais modernos cenários existentes, utilizados pela Organização das Nações Unidas para gerar o último relatório sobre mudanças climáticas (IPCC 2014). Houve melhora dos métodos de projeção do quarto para o quinto relatório no que se refere a precipitação e simulações do nível do oceano, no entanto a principal diferença é que os cenários RCP incluem uma maior faixa de variação dos gases em relação aos cenários SRES (usados no quarto relatório) e também adicionam cenários de políticas de clima (IPCC 2014). Em especial, o RCP2.6 é um cenário novo que inclui o efeito das ações esperadas nas convenções sobre clima atuais, por isso os resultados com este cenário podem ser mais otimistas do que os obtidos em outros trabalhos que usaram os cenários antigos. No entanto, o nível de confiança nos cenários aumentou e as incertezas diminuíram o que reforça a plausibilidade dos cenários RCP.

\section{Uso da terra e deslocamento na paisagem}

A possibilidade de deslocamento na paisagem para novas áreas é uma das principais adaptações discutidas como forma de se adequar a mudança do clima (Bakkenes et al. 2002, Huntley et al. 2006, McClean et al. 2006, Albouy et al. 2012, Bellard et al. 2014). No entanto, a movimentação para uma área nova é uma tarefa difícil para algumas espécies que possuem mobilidade reduzida e dificuldade de se deslocar por áreas da matriz na paisagem (Rothermel and Semlitsch 2002, Vasudev and Fletcher 2015). Mesmo estimativas da movimentação que as espécies são capazes de realizar (Barbet-Massin et al. 2012, Faleiro et al. 2013) se mostram irreais em nosso caso, pois consideram que elas irão atravessar vastas áreas sem adequabilidade climática ou mesmo sem vegetação o que é particularmente difícil para primatas arborícolas (Vasudev and Fletcher 2015). O caso mais conhecido de expansão da área original em 
animal do grupo é o do sagui comum (Callithrix jacchus) que ocupa áreas no sudeste do Brasil, o que só foi possível porque foi transportado por pessoas (Ruiz-Miranda et al. 2000).

Nós encontramos que a restrição da migração das espécies para novas áreas foi uma variável que teve alta influência no tamanho da área ocupada por ela no futuro. Isso significa que as espécies terão que se deslocar consideravelmente para se adaptar às novas condições do clima e manterem o mesmo vigor populacional que apresentam atualmente. Um exemplo é o Sapajus xanthosternos, que teve a maior parte da sua distribuição potencial futura projetada para o sul.

A mudança no uso da terra é o principal fator para explicar a diminuição da área projetada das espécies para o futuro. Esse é um efeito que já foi encontrada em outro trabalho, que comparou dados de taxas de desmatamento com dados simulados de mudança climáticas e viu que em média o efeito do desmatamento na perda de área das espécies foi mais que o dobro do que o efeito das mudanças climáticas (Malcolm et al. 2006). É importante investir em impedir novos desmatamentos no Cerrado para diminuir o ritmo de mudança no uso da terra e evitar o cenário projetado de degradação da biota.

O presente trabalho é um dos poucos que utilizou estimativas robustas de desmatamento e mudanças no uso da terra para o futuro em estimativas de perda de área de táxons. Uma das exceções é o trabalho de Barbet-Massin e colaboradores (BarbetMassin et al. 2012), que analisaram uma projeção de mudança da cobertura do solo e compararam com previsões de mudanças climáticas para aves europeias. Entretanto, a prática mais usual é a de realizar simulações para o futuro e usar estimativa de uso da terra no presente (Thuiller et al. 2006, Gadsden et al. 2012). O modelo de mudança de 
uso da terra utilizado emprega a ideia que a taxa de conversão de terra continuará no mesmo ritmo existente entre 2002 e 2008.

\section{Graus de ameaca}

O risco de extinção de espécies é provavelmente o tema mais pesquisado em estudos sobre mudança climática. Um dos primeiros trabalhos feitos com esse intuito usando modelos climáticos se estimou que de 15 a $37 \%$ das espécies analisadas estariam ‘comprometidas com a extinção' (Thomas et al. 2004). De 25 a $42 \%$ das plantas africanas devem perder toda a área até 2085 (McClean et al. 2006) e no Brasil, 43\% das plantas da Amazônia (Miles et al. 2004) e de 56 a 76\% das plantas do Cerrado (Siqueira and Peterson 2003) devem perder grande parte da sua área nos próximos anos. De 1,3 a $30 \%$ das aves do hemisfério ocidental e 400 a 550 aves terrestres do mundo estão previstas de se tornarem extintas até o final de 2100 (Sekercioglu et al. 2008). 25\% das espécies de aves europeias estão em risco de extinção pela significativa redução da sua área (Huntley et al. 2006). No pior cenário modelado 4\% dos mamíferos africanos devem se tornar extintos e 37,5\% criticamente ameaçados depois de 2080 (Thuiller et al. 2006). .Um estudo com modelos fisiológicos calculou que os lagartos do mundo irão perder $20 \%$ das espécies existentes e devem sofrer extinções locais da ordem de $39 \%$ (Sinervo et al. 2010). Um trabalho analisando todos os hotspots calculou que, em média, 11,6\% (1-43\%) das espécies endêmicas dessas unidades irão desaparecer em 100 anos (Malcolm et al. 2006). Uma revisão recente dos trabalhos publicados no tema calculou uma média de 7,9\% de risco de extinção em todos os trabalhos, com a América do Sul apresentando uma média muito mais elevada, em torno de 23\% (Urban 2015).

Outra prática comum em trabalhos que analisam o efeito de mudanças climáticas na distribuição das espécies é calcular qual será o status de ameaça que cada táxon terá 
segundo os critérios da IUCN (IUCN 2001). Essa prática foi reproduzida para estimar os graus de ameaça de vários grupos: de 50 a $60 \%$ dos mamíferos africanos deverão ficar ameaçados até 2050(Thuiller et al. 2006); de 25 a 55\% das aves do hemisfério ocidental até 2100 (Sekercioglu et al. 2008); de 65 a $70 \%$ das plantas da região do Cabo até 2020 (Bomhard et al. 2005). Em um trabalho usando uma coletânea de grupos e estimativas de risco de extinção baseadas nas categorias da IUCN (quanto mais alta a categoria maior o risco de extinção), se estimou que de 34 a 58\% das espécies estão com risco de extinção na condição sem migração (Thomas et al. 2004). As aves endêmicas do Cerrado não devem modificar o atual status de ameaça (Marini et al. 2009).

No entanto, essa prática de usar as categorias da IUCN para o futuro foi criticada por fazer constante uso de modificações nos critérios da IUCN, o que na verdade tornam as estimativas de graus de ameaças apenas projeções que não se relacionam com a atual lista vermelha de espécies ameaçadas (Akçakaya et al. 2006). Uma das modificações mais comuns é do critério A3 que expressa porcentagens de perda de área em dez anos para o futuro, entretanto alguns estudos modificaram para cinquenta anos para o futuro (Thomas et al. 2004, Bomhard et al. 2005, Sekercioglu et al. 2008). O atual estudo não modificou os critérios da IUCN utilizados, fazendo uma estimativa com critérios mais conservadores que em outros estudos, porém fiéis aos propostos pela IUCN e comparáveis com as atuais listas vermelhas.

Nós encontramos que quatro espécies com área no Cerrado possuem risco de se extinguir da região do Cerrado até o final de 2050 (Alouatta belzebul, Aotus azarae infulatus, Mico melanurus e Sapajus xanthosternos), com o RCP 8.5 sendo o cenário de previsões mais pessimistas. Alouatta belzebul e Aotus infulatus são espécies com boa parte da sua distribuição em áreas da Amazônia e eles tiveram uma mudança na 
distribuição para o oeste deixando completamente as áreas ocupadas no Cerrado e acompanhando a mudança prevista para o centro da Amazônia (Salazar et al. 2007). Sapajus xanthosternos é uma espécie com distribuição mais restrita e área atual no Cerrado em uma parte da Bahia e ela terá um deslocamento de habitat para o sul nos cenários pessimistas e para o nordeste nos cenários otimistas, deixando o Cerrado. Mico melanurus é a espécie com o maior risco de extinção de todas, pois poderá se extinguir de toda a área de sua distribuição, mesmo na condição de deslocamento ilimitado e mapa total. Também é importante ressaltar a significativa diminuição da área projetada no Cerrado de Sapajus libidinosus e Callithrix jacchus que são associadas ao ambiente mais aberto e com área na Caatinga. Essas são espécies que não possuem risco de extinção atual e são abundantes em boa parte do Cerrado, mas devem se tornar raras por causa das mudanças climáticas e uso da terra.

As maiores ameaças para os primatas do Cerrado atualmente são a perda de área disponível e a caça (Machado et al. 2008a). A caça é um fator que não foi parte do escopo das projeções deste estudo e ela possui um padrão mais complexo e difícil de prever. No entanto, pode ter um efeito devastador em algumas populações que são traficadas para a venda como animais de estimação (Kierulff et al. 2004). O aumento na taxa de mortalidade devido à caça nas populações de Sapajus xanthosternos faz com que a probabilidade de extinção aumente substancialmente (Paglia 2003).

Por fim, ressaltamos que a medida mais eficaz para evitar futuras extinções regionais da primatofauna é a diminuição do desmatamento no Cerrado. Estudos futuros devem analisar o efeito de mudanças climáticas e uso da terra em primatas de outras regiões, principalmente do Chaco que apresentou casos preocupantes de deslocamentos de espécies. É importante utilizar projeções de desmatamento mais abrangentes que 
permitam usar as projeções para todo o continente sul-americano para entender esses efeitos sobre todos os platirrinos. 


\section{REFERÊNCIAS BIBLIOGRAFICAS}

Ab’Saber, A. N. 1977. Os domínios morfoclimáticos na América do Sul. Geomorfologia 52:1-23.

Aguiar, L. M., N. R. Reis, G. Ludwig, and V. J. Rocha. 2003. Dieta, área de vida, vocalizações e estimativas populacionais de Alouatta guariba em um remanescente florestal no norte do estado do Paraná. Neotropical Primates 11:78-86.

Akçakaya, H. R., S. H. M. Butchart, G. M. Mace, S. N. Stuart, and C. Hilton-Taylor. 2006. Use and misuse of the IUCN Red List Criteria in projecting climate change impacts on biodiversity. Global Change Biology 12:2037-2043.

Albouy, C., F. Guilhaumon, M. B. Araújo, D. Mouillot, and F. Leprieur. 2012. Combining projected changes in species richness and composition reveals climate change impacts on coastal Mediterranean fish assemblages. Global Change Biology 18:2995-3003.

Alfaro, J. L. W., J. D. J. Silva, and A. B. Rylands. 2012a. How different are robust and gracile capuchin monkeys? An argument for the use of Sapajus and Cebus. American Journal of Primatology 74:273-286.

Alfaro, J. W. L., J. D. E. S. E. Silva, and A. B. Rylands. 2012b. How Different Are Robust and Gracile Capuchin Monkeys? An Argument for the Use of Sapajus and Cebus. American Journal of Primatology 74:273-286.

Allouche, O., A. Tsoar, and R. Kadmon. 2006. Assessing the accuracy of species distribution models: prevalence, kappa and the true skill statistic (TSS). Journal of Applied Ecology 43:1223-1232.

Andelman, S. J. and W. F. Fagan. 2000. Umbrellas and flagships: efficient conservation surrogates or expensive mistakes? Proceedings of the National Academy of Sciences of the United States of America 97:5954-5959.

Araujo, M. B. 2009. Climate Change and Spatial Conservation Planning. Page 328 in A. Moilanen, K. A. Wilson, and H. P. Possingham, editors. Spatial conservation prioritization : quantitative methods and computational tools. Oxford Universtiy Press, Oxford, UK.

Araújo, M. B., R. J. Whittaker, R. J. Ladle, and M. Erhard. 2005. Reducing uncertainty in projections of extinction risk from climate change. Global Ecology and Biogeography 14:529-538.

Araújo, R. M. D., M. B. D. Souza, and C. R. Ruiz-miranda. 2008. Densidade e tamanho populacional de mamíferos cinegéticos em duas Unidades de Conservação do Estado do Rio de Janeiro, Brasil. Iheringia, Série Zoológica 98:391-396.

Bakkenes, M., J. R. M. Alkemade, F. Ihle, R. Leemans, and J. B. Latour. 2002. Assessing effects of forecasted climate change on the diversity and distribution of European higher plants for 2050. Global Change Biology 8:390-407.

Ball, I. R., H. P. Possingham, and M. Watts. 2009. Marxan and relatives: Software for spatial conservation prioritization. Pages 185-195 in A. Moilanen, K. A. Wilson, and H. P. Possingham, editors. Spatial conservation prioritization: Quantitative methods and computational tools. Oxford University Press, Oxford, UK. 
Barbet-Massin, M., W. Thuiller, and F. Jiguet. 2012. The fate of European breeding birds under climate, land-use and dispersal scenarios. Global Change Biology 18:881-890.

Batalha-Filho, H., J. Fjeldså, P.-H. Fabre, and C. Y. Miyaki. 2012. Connections between the Atlantic and the Amazonian forest avifaunas represent distinct historical events. Journal of Ornithology 154:41-50.

Bates, D., M. Maechler, B. Bolker, and S. Walker. 2014. lme4: Linear mixed-effects models using Eigen and S4. Journal of Statistical software.

Bellard, C., C. Bertelsmeier, P. Leadley, W. Thuiller, and F. Courchamp. 2012. Impacts of climate change on the future of biodiversity. Ecology Letters 15:365-377.

Bellard, C., C. Leclerc, B. Leroy, M. Bakkenes, S. Veloz, W. Thuiller, and F. Courchamp. 2014. Vulnerability of biodiversity hotspots to global change. Global Ecology and Biogeography 23:1376-1386.

Bitetti, M. S. 2001. Home-range use by the tufted capuchin monkey (Cebus apella nigritus) in a subtropical rainforesr of Argentina. Journal of Zoology (London) 253:33-45.

Bomhard, B., D. M. Richardson, J. S. Donaldson, G. O. Hughes, G. F. Midgley, D. C. Raimondo, A. G. Rebelo, M. Rouget, and W. Thuiller. 2005. Potential impacts of future land use and climate change on the Red List status of the Proteaceae in the Cape Floristic Region, South Africa. Global Change Biology 11:1452-1468.

Boria, R. A., L. E. Olson, S. M. Goodman, and R. P. Anderson. 2014. Spatial filtering to reduce sampling bias can improve the performance of ecological niche models. Ecological Modelling 275:73-77.

Boubli, J. P. and M. G. Lima. 2009. Modeling the geographical distribution and fundamental niches of Cacajao spp. and Chiropotes israelita in northwestern Amazonia via a Maximum Entropy algorithm. International Journal of Primatology 30:217-228.

Brito, D. and C. E. V. Grelle. 2006. Estimating Minimum Area of Suitable Habitat and Viable Population Size for the Northern Muriqui (Brachyteles hypoxanthus). Biodiversity and Conservation 15:4197-4210.

Brooks, D. R. and M. G. P. van Veller. 2003. Critique of PAE as a method of historical biogeography. Journal of Biogeography 30:819-825.

Buckner, J. C., J. W. Lynch Alfaro, A. B. Rylands, and M. E. Alfaro. 2014. Biogeography of the marmosets and tamarins (Callitrichidae). Molecular phylogenetics and evolution 82 Pt B:413-425.

Buisson, L., W. Thuiller, N. Casajus, S. Lek, and G. Grenouillet. 2010. Uncertainty in ensemble forecasting of species distribution. Global Change Biology 16:11451157.

Carine, M. A., C. J. Humphries, I. R. Guma, J. A. Reyes-Betancort, and A. Santos Guerra. 2009. Areas and algorithms: evaluating numerical approaches for the delimitation of areas of endemism in the Canary Islands archipelago. Journal of Biogeography 36:593-611.

Carnaval, A. C., M. J. Hickerson, C. F. B. Haddad, M. T. Rodrigues, and C. Moritz. 2009. Stability predicts genetic diversity in the Brazilian Atlantic Forest hotspot. Science 323:785-789.

Carnaval, A. C. and C. Moritz. 2008. Historical climate modelling predicts patterns of current biodiversity in the Brazilian Atlantic forest. Journal of Biogeography 35:1187-1201. 
Casagranda, M. D., L. Taher, and C. a. Szumik. 2012. Endemicity analysis, parsimony and biotic elements: A formal comparison using hypothetical distributions. Cladistics 28:645-654.

Chapin, F. S., P. A. Matson, and H. A. Mooney. 2002. Principles of Terrestrial Ecosystem Ecology. Springer Science, New York.

Chiarello, A. G. and F. R. Melo. 2001. Primate population densities and sizes in Atlantic Forest remnants of northern Espirito Santo, Brazil. International Journal of Primatology 22:379-396.

Codenotti, T. L., V. M. Silva, V. J. Albuquerque, E. V. Camargo, and R. M. M. Silveira. 2002. Distribuição e situação atual de conservação de Alouatta caraya (Humboldt, 1812) no Rio Grande do Sul, Brasil. Neotropical Primates 10:132141.

Cortés-Ortiz, L., E. Bermingham, C. Rico, E. Rodríguez-Luna, I. Sampaio, and M. Ruiz-García. 2003. Molecular systematics and biogeography of the Neotropical monkey genus, Alouatta. Molecular phylogenetics and evolution 26:64-81.

Costa, L. P. 2003. The historical bridge between the Amazon and the Atlantic Forest of Brazil: A study of molecular phylogeography with small mammals. Journal of Biogeography 30:71-86.

Coulon, A., J. F. Cosson, J. M. Angibault, B. Cargnelutti, M. Galan, N. Morellet, E. Petit, S. Aulagnier, and A. J. Hewison. 2004. Landscape connectivity influences gene flow in a roe deer population inhabiting a fragmented landscape: an individual-based approach. Molecular ecology 13:2841-2850.

Crisci, J. V. 2001. The voice of historical biogeography. Journal of Biogeography 28:157-168.

Diniz-Filho, J. A. F., L. M. Bini, M. P. Pinto, L. C. Terribile, G. de Oliveira, C. M. Vieira, D. Blamires, B. de Souza Barreto, P. Carvalho, T. F. L. V. B. Rangel, N. M. Tôrres, and R. P. Bastos. 2008. Conservation planning: a macroecological approach using the endemic terrestrial vertebrates of the Brazilian Cerrado. Oryx 42:567.

Eastman, J. R. 2009. Guide to GIS and Image Processing. Clark University, Worcester, Massachusetts.

Eiten, G. 1972. The Cerrado Vegetation of Brazil. Botanical Review 38:201-341.

Eiten, G. 1994. Vegetação do Cerrado. Pages 17-73 in M. Pinto, editor. Cerrado: caracterização, ocupação e perspectivas. Editora da Universidade de Brasília, Brasília, DF.

Elith, J., C. H. Graham, R. P. Anderson, M. Dudık, S. Ferrier, A. Guisan, R. J. Hijmans, F. Huettmann, J. R. Leathwick, A. Lehmann, J. Li, L. G. Lohmann, B. A. Loiselle, G. Manion, C. Moritz, M. Nakamura, Y. Nakazawa, J. M. Overton, A. T. Peterson, S. J. Phillips, K. Richardson, R. Scachetti-pereira, R. E. Schapire, J. Soberón, S. Williams, M. S. Wisz, and N. E. Zimmermann. 2006. Novel methods improve prediction of species' distributions from occurrence data. Ecography 29:129-151.

Elith, J. and J. Leathwick. 2009a. The Contribution of Species Distribution Modelling to Conservation Prioritization. Pages 70-93 in A. Moilanen, K. A. Wilson, and H. P. Possingham, editors. Spatial Conservation Prioritization. Oxford University Press, Oxford.

Elith, J. and J. R. Leathwick. 2009b. Species Distribution Models: Ecological Explanation and Prediction Across Space and Time. Annual Review of Ecology, Evolution, and Systematics 40:677-697. 
Engler, R., A. Guisan, and L. Rechsteiner. 2004. An improved approach for predicting the distribution of rare and endangered species from occurrence and pseudoabsence data. Journal of Applied Ecology 41:263-274.

Estrada, A. 2006. Human and Non-human primate co-existence in the Neotropics: a preliminary view of some agricultural practices as a complement for primate conservation. Ecological and Environmental Anthropology 2:17-29.

Fagan, W. F. and E. E. Holmes. 2006. Quantifying the extinction vortex. Ecology Letters 9:51-60.

Faleiro, F. V., R. B. Machado, and R. D. Loyola. 2013. Defining spatial conservation priorities in the face of land-use and climate change. Biological Conservation 158:248-257.

Fernandez-Duque, E. 2007. Aotinae: Social monogamy in the only nocturnal haplorhines. Pages 139-154 in C. J. Campbell, A. Fuentes, K. C. Mackinnon, M. Panger, and S. K. Bearder, editors. Primates in Perspective. Oxford University Press, Oxford, UK.

Fernandez-Duque, E., M. Rotundo, and C. Sloan. 2001. Density and Population Structure of Owl Monkeys ( Aotus azarai ) in the Argentinean Chaco. American Journal of Primatology 53:99-108.

Fleagle, J. G. 1999. Primate Adaptation and Evolution. 2nd edition. Elsevier Academic Press.

Foley, J. A., I. C. Prentice, N. Ramankutty, S. Levis, D. Pollard, S. Sitch, and A. Haxeltine. 1996. An integrated biosphere model of land surface processes, terrestrial carbon balance, and vegetation dynamics. Global Biogeochemical Cycles 10:603-628.

Freitag, S. and A. S. Van Jaarsveld. 1998. Sensitivity of selection procedures for priority conservation areas to survey extent, survey intensity and taxonomic knowledge. Proceedings of the Royal Society B: Biological Sciences 265:14751482.

Gadsden, H., C. Ballesteros-Barrera, O. Hinojosa de la Garza, G. Castañeda, C. GarcíaDe la Peña, and J. A. Lemos-Espinal. 2012. Effects of land-cover transformation and climate change on the distribution of two endemic lizards, Crotaphytus antiquus and Sceloporus cyanostictus, of northern Mexico. Journal of Arid Environments 83:1-9.

Garcia, R. A., N. D. Burgess, M. Cabeza, C. Rahbek, and M. B. Araújo. 2012. Exploring consensus in 21st century projections of climatically suitable areas for African vertebrates. Global Change Biology 18:1253-1269.

Geerts, B. 2003. Empirical estimation of the monthly-mean daily temperature range. Theoretical and Applied Climatology 74:145-165.

Gladstone, W. and J. Davis. 2003. Reduced survey intensity and its consequences for marine reserve selection. Biodiversity and Conservation 12:1525-1536.

Goldani, A., G. S. Carvalho, and J. C. Bicca-Marques. 2006. Distribution patterns of neotropical primates (Platyrrhini) based on Parsimony Analysis of Endemicity. Brazilian journal of biology 66:61-74.

Grelle, C. E. V. and R. Cerqueira. 2006. Determinantes da distribuição geográfica de Callithrix flaviceps (Thomas) (Primates, Callitrichidae). Revista Brasileira de Zoologia 2006:414-420.

Grenouillet, G., L. Buisson, N. Casajus, and S. Lek. 2011. Ensemble modelling of species distributions: the effects of geographical and environmental ranges. Ecography 34:9-17. 
Guisan, A., O. Broennimann, R. Engler, M. Vust, N. G. Yoccoz, A. Lehmann, and N. E. Zimmermann. 2006. Using Niche-Based Models to Improve the Sampling of Rare Species. Conservation Biology 20:501-511.

Hanley, J. A. and B. J. McNeil. 1982. The meaning and use of the area under a Receiver Operating Characteristic (ROC) curve. Radiology 143:29-36.

Hanya, G., P. Stevenson, M. van Noordwijk, S. Te Wong, T. Kanamori, N. Kuze, S.-i. Aiba, C. A. Chapman, and C. van Schaik. 2011. Seasonality in fruit availability affects frugivorous primate biomass and species richness. Ecography 34:10091017.

Hastie, T., R. Tibshirani, and J. Friedman. 2009. Elements of Statistical Learning - Data Mining, Inference and Prediction. 2nd edition. Springer, California, US.

Hausdorf, B. 2002. Units in Biogeography. Systematic Biology 51:648-652.

Hausdorf, B. and C. Hennig. 2003. Biotic Element Analysis in Biogeography. Systematic Biology 52:717-723.

Hausdorf, B. and C. Hennig. 2004. Does vicariance shape biotas Biogeographical tests of the vicariance model in the north-west European land snail fauna. Journal of Biogeography 31:1751-1757.

Heikkinen, R. K., M. Luoto, M. B. Araújo, R. Virkkala, W. Thuiller, and M. T. Sykes. 2006. Methods and uncertainties in bioclimatic envelope modelling under climate change. Progress in Physical Geography 30:751-777.

Hennig, C. and B. Hausdorf. 2015. prabclus: Functions for Clustering of Presenceabsence, abundance and multilocus genetic data.

Hijmans, R. 2015. Raster: Geographic data analysis and modeling.

Hijmans, R. J., S. E. Cameron, J. L. Parra, P. G. Jones, and A. Jarvis. 2005. Very high resolution interpolated climate surfaces for global land areas. International Journal of Climatology 25:1965-1978.

Hirsch, A., L. G. Dias, L. d. O. Martins, R. F. Campos, E. C. Landau, and N. A. T. Resende. 2002. BDGEOPRIM - Database of geo-referenced localities of Neotropical Primates. Neotropical Primates 10:79-84.

Hole, D. G., S. G. Willis, D. J. Pain, L. D. Fishpool, S. H. Butchart, Y. C. Collingham, C. Rahbek, and B. Huntley. 2009. Projected impacts of climate change on a continent-wide protected area network. Ecology Letters 12:420-431.

Hugall, A., C. Moritz, A. Moussalli, and J. Stanisic. 2002. Reconciling paleodistribution models and comparative phylogeography in the Wet Tropics rainforest land snail Gnarosophia bellendenkerensis (Brazier 1875). Proceedings of the National Academy of Sciences of the United States of America 99:6112-6117.

Huntley, B., Y. C. Collingham, R. E. Green, G. M. Hilton, C. Rahbek, and S. G. Willis. 2006. Potential impacts of climatic change upon geographical distribution of birds. Ibis 148:8-28.

Hutchinson, G. E. 1958. Concluding remarks. Pages 415-427 Cold Spring Harbor Symposia on Quantitative Biology.

IBGE. 2004. Mapa de Biomas do Brasil. Escala 1:5.000.000.

IPCC. 2007. Summary for Policymakers. Pages 7-22 in M. L. Parry, O. F. Canziani, J. P. Palutikof, P. J. van der Linden, and C. E. Hanson, editors. Climate Change 2007: Impacts, Adaptation and Vulnerability. Contribution of Working Group II to the Fourth Assessment Report on the Intergovernamental Panel on Climate Change. Cambridge University Press, Cambridge, UK.

IPCC. 2014. Climate Change 2014: Synthesis report.

IUCN. 2001. IUCN Red List Categories and Criteria Version 3.1. Page 30. 
IUCN. 2003. Guidelines for Application of IUCN Red List Criteria at Regional Levels Version 3.0. IUCN, Gland, Switzerland and Cambridge, UK.

Jennings, M. D. 2000. Gap analysis concepts, methods, and recent results. Landscape Ecology 15:5-20.

Jiménez-Valverde, A. and J. M. Lobo. 2007. Threshold criteria for conversion of probability of species presence to either-or presence-absence. Acta Oecologica 31:361-369.

Jones, C. B. 1987. Evidence supporting the Pleistocene Forest Refuge hypothesis for primates. Biotropica 19:373-375.

Kamilar, J. M. 2009. Environmental and geographic correlates of the taxonomic structure of primate communities. American Journal of Physical Anthropology 139:382-393.

Kay, R. F. 2015. Biogeography in deep time - What do phylogenetics, geology, and paleoclimate tell us about early platyrrhine evolution? Molecular phylogenetics and evolution 82 Pt B:358-374.

Kay, R. F. and R. H. Madden. 1997. Mammals and rainfall: paleoecology of middle miocene at La Venta (Colombia, South America). Journal of Human Evolution 32:161-199.

Kay, R. F., R. H. Madden, C. Van Schaik, and D. Higdon. 1997. Primate species richness is determined by plant productivity: Implications for conservation. Proceedings of the National Academy of Sciences 94:13023-13027.

Kierulff, M. C. M., G. R. Santos, G. R. Canale, C. E. Guidorizzi, and C. Cassano. 2004. The use of camera-traps in a sourvey of the buff-headed capuchin monkey. Neotropical Primates 12:56-59.

Kiesling, N. M. J., S. V. Yi, K. Xu, F. Gianluca Sperone, and D. E. Wildman. 2015. The tempo and mode of New World monkey evolution and biogeography in the context of phylogenomic analysis. Molecular phylogenetics and evolution $82 \mathbf{P t}$ B:386-399.

Kinzey, W. G. 1982. Distribution of Primates and forest refuges. Pages 455-482 in G. T. Prance, editor. Biological Diversification in the Tropics. Columbia University Press, New York, NY.

Klink, C. and R. B. Machado. 2005. Conservation of the Brazilian Cerrado. Conservation Biology 19:707-713.

Kucharik, C. J., J. A. Foley, C. Delire, V. A. Fisher, M. T. Coe, J. D. Lenters, C. Young-Molling, N. Ramankutty, J. M. Norman, and S. T. Gower. 2000. Testing the performance of a dynamic global ecosystem model: Water balance, carbon balance, and vegetation structure. Global Biogeochemical Cycles 14:795-825.

Lamm, K. S. and B. D. Redelings. 2009. Reconstructing ancestral ranges in historical biogeography: properties and prospects. Journal of Systematics and Evolution 47:369-382.

Le Maitre, D. C., W. Thuiller, and L. Schonegevel. 2008. Developing an approach to defining the potential distributions of invasive plant species: a case study of Hakea species in South Africa. Global Ecology and Biogeography 17:569-584.

Lenth, R. V. and M. Hervã. 2015. lsmeans: Least-Square Means.

Liu, C., P. M. Berry, T. P. Dawson, and R. G. Pearson. 2005. Selecting thresholds of occurrence in the prediction of species distributions. Ecography 28:385-393.

Loarie, S. R., P. B. Duffy, H. Hamilton, G. P. Asner, C. B. Field, and D. D. Ackerly. 2009. The velocity of climate change. Nature 462:1052-1055.

Lynch Alfaro, J. W., J. P. Boubli, L. E. Olson, A. Di Fiore, B. Wilson, G. A. GutiérrezEspeleta, K. L. Chiou, M. Schulte, S. Neitzel, V. Ross, D. Schwochow, M. T. T. 
Nguyen, I. Farias, C. H. Janson, and M. E. Alfaro. 2012. Explosive Pleistocene range expansion leads to widespread Amazonian sympatry between robust and gracile capuchin monkeys. Journal of Biogeography 39:272-288.

Lynch Alfaro, J. W., L. Cortes-Ortiz, A. Di Fiore, and J. P. Boubli. 2015. Special issue: Comparative biogeography of Neotropical primates. Molecular phylogenetics and evolution 82 Pt B:518-529.

Machado, A. B. M., G. M. Drummond, and A. P. Paglia. 2008a. Livro Vermelho da Fauna Brasileira ameaçada de extinção, volume 2. MMA, Brasília-DF.

Machado, R. B., L. M. S. Aguiar, A. A. J. F. Castro, C. C. Nogueira, and M. B. R. Neto. 2008b. Caracterização da Fauna e Flora do Cerrado.in F. Faleiro and A. Neto, editors. Savanas - desafios e estratégias para o equilíbrio entre sociedade, agronegócio e recursos naturais. Embrapa Cerrados, Planaltina- DF.

Machado, R. B., M. B. R. Neto, P. G. P. Pereira, E. F. Caldas, D. A. Gonçalves, N. S. Santos, K. Tabor, and M. Steininger. 2004. Estimativas de perda da área do Cerrado brasileiro. Brasília-DF.

Malcolm, J. R., C. Liu, R. P. Neilson, L. Hansen, and L. E. E. Hannah. 2006. Global Warming and Extinctions of Endemic Species from Biodiversity Hotspots. Conservation Biology 20:538-548.

Marini, M. Â., M. Barbet-Massin, L. E. Lopes, and F. Jiguet. 2010a. Predicting the occurrence of rare Brazilian birds with species distribution models. Journal of Ornithology 151:857-866.

Marini, M. Â., M. Barbet-Massin, J. Martinez, N. P. Prestes, and F. Jiguet. 2010b. Applying ecological niche modelling to plan conservation actions for the Redspectacled Amazon (Amazona pretrei). Biological Conservation 143:102-112.

Marini, M. Ã. N., M. Barbet-Massin, L. E. Lopes, and F. D. R. Jiguet. 2009. Predicted Climate-Driven Bird Distribution Changes and Forecasted Conservation Conflicts in a Neotropical Savanna. Conservation Biology 23:1558-1567.

Marmion, M., M. Parviainen, M. Luoto, R. K. Heikkinen, and W. Thuiller. 2009. Evaluation of consensus methods in predictive species distribution modelling. Diversity and Distributions 15:59-69.

McClean, C. J., J. C. Lovett, W. Küper, L. Hannah, J. H. Sommer, W. Barthlott, M. Termansen, G. F. Smith, S. Tokumine, and J. R. D. Taplin. 2006. African plant diversity and climate change. Annals of the Missouri Botanical Garden 92:139152.

Meller, L., M. Cabeza, S. Pironon, M. Barbet-Massin, L. Maiorano, D. Georges, and W. Thuiller. 2014. Ensemble distribution models in conservation prioritization: from consensus predictions to consensus reserve networks. Diversity \& distributions 20:309-321.

Miles, L., A. Grainger, and O. L. Phillips. 2004. The impact of global climate change on tropical forest biodiversity in Amazonia. Global Ecology and Biogeography 13:553-565.

MMA. 2002. Biodiversidade brasileira: avaliação e identificação de áreas e ações prioritárias para conservação, utilização sustentável e repartição dos benefícios da biodiversidade nos biomas brasileiros. Page 404 in SBF, editor. MMA, Brasília- DF.

MMA. 2006. Mapa de Áreas Prioritárias do Cerrado em formato SHAPE. Brasília-DF.

MMA. 2014. Portaria 444, de 17 de dezembro de 2014. Pages 121-126 in MMA, editor. 444, DOU.

MMA/IBAMA. 2009. Relatório técnico de monitoramento do desmatamento no bioma Cerrado, 2002 a 2008: Dados revisados. MMA, Brasília-DF. 
MMA/IBAMA. 2011a. Monitoramento do desmatamento nos biomas brasileiros por satélite: acordo de cooperação técnica MMA/IBAMA. Monitoramento do bioma Cerrado 2008-2009. MMA, Brasília-DF.

MMA/IBAMA. 2011b. Monitoramento do desmatamento nos biomas brasileiros por satélite: acordo de cooperação técnica MMA/IBAMA. Monitoramento do bioma Cerrado 2009-2010. MMA, Brasília-DF.

Morrone, J. J. 1994. On the identification of areas of endemism. Systematic Biology 43:438-441.

Myers, N., R. A. Mittermeier, C. G. Mittermeier, G. A. B. Fonseca, and J. Kent. 2000. Biodiversity hotspots for conservation priorities. Nature 403:853-858.

Nihei, S. S. 2006. Misconceptions about parsimony analysis of endemicity. Journal of Biogeography 33:2099-2106.

Nogueira, C., S. Ribeiro, G. C. Costa, and G. R. Colli. 2011. Vicariance and endemism in a Neotropical savanna hotspot: distribution patterns of Cerrado squamate reptiles. Journal of Biogeography 38:1907-1922.

Oliveira-Filho, A. T. and J. A. Ratter. 1995. A study of the origin of central brazilian forests by the analysis of plant species distribution patterns. Edinburgh Journal of Botany 52:141-194.

Oliveira, R. C. R. d., A. S. Coelho, and F. R. Melo. 2003. Estimativa de densidade populacional de Sauá (Callicebus nigrifrons) em um fragmento de mata em regeneração, Viçosa, Minas Gerais, Brasil Neotropical Primates 11:91-93.

Otto-Bliesner, B. L. 2006. Simulating Arctic Climate Warmth and Icefield Retreat in the Last Interglaciation. Science 311:1751-1753.

Paglia, A. P. 2003. Análises de viabilidade populacional: quantos indivíduos? Serão eles suficientes? Estudo de caso para espécies ameaçadas da mata atlântica do sul da Bahia. Conservation International.

Paglia, A. P., G. A. B. Fonseca, A. B. Rylands, G. Herrmann, L. M. S. Aguiar, A. G. Chiarello, Y. L. R. Leite, L. P. Costa, S. Siciliano, M. C. M. Kierulff, S. L. Mendes, V. d. C. Tavares, R. A. Mittermeier, and J. L. Patton. 2012. Lista Anotada dos Mamíferos do Brasil. $2^{a}$ edition. Conservation International, Arlington, VA.

Parmesan, C. 2006. Ecological and Evolutionary Responses to Recent Climate Change. Annual Review of Ecology, Evolution, and Systematics 37:637-669.

Peterson, A. T. and J. Soberón. 2012. Species Distribution Modeling and Ecological Niche Modeling: Getting the Concepts Right. Natureza \& Conservação 10:102107.

Peterson, A. T., J. Soberón, R. G. Pearson, R. P. Anderson, E. Martinez-Meyer, M. Nakamura, and M. B. Araujo. 2011. Ecological Niches and Geographic Distributions. Princeton University Press, Princeton, USA.

Peterson, A. T., J. Soberon, and V. Sánchez-Cordero. 1999. Conservatism of ecological niche in Evolutionary Time. Nature 285:1265-1267.

Petit, J. R., J. Jouzel, D. Raynaud, N. I. Barkov, J.-M. Barnola, I. Basile, M. Bender, J. Chappellaz, M.Davis, G. Delaygue, M. Delmotte, V. M. Kotlyakov, M. Legrand, V. Y. Lipenkov, C. Lorius, L. Pépin, C. Ritz, E. Saltzman, and M. Stievenard. 1999. Climate and atmospheric history of the past 420,000 years from the Vostok ice core, Antarctica. Nature 399:429-436.

Phillips, S. J., R. P. Anderson, and R. E. Schapire. 2006. Maximum entropy modeling of species geographic distributions. Ecological Modelling 190:231-259. 
Phillips, S. J., M. Dudik, and R. E. Schapire. 2004. A Maximum Entropy Approach to Species Distribution Modeling. Pages 1-8 in Proceedings of the 21st International Conference on Machine Learning, Banff, Canada.

Pinheiro, J., D. Bates, S. DebRoy, D. Sarkar, and R. c. team. 2015. \{nlme\}: Linear and nonlinear mixed effects models.

Pinto, M. P. and C. E. V. Grelle. 2009. Reserve selection and persistence: complementing the existing Atlantic Forest reserve system. Biodiversity and Conservation 18:957-968.

Pinto, M. P., S. Silva-Junior Jde, A. A. Lima, and C. E. Grelle. 2014. Multi-scales analysis of primate diversity and protected areas at a megadiverse region. PLoS ONE 9:e105205.

Polechová, J. and D. Storch. 2008. Ecological Niche. Pages 1088-1097 in S. E. Jorgensen and B. D. Fath, editors. Enciclopedia of Ecology. Elsevier Academic Press, Oxford.

Posadas, P., J. V. Crisci, and L. Katinas. 2006. Historical biogeography: A review of its basic concepts and critical issues. Journal of Arid Environments 66:389-403.

Possingham, H. P., I. R. Ball, and S. Andelman. 2000. Mathematical methods for identifying representative reserve networks. Page 322 in S. Fersson and M. Burgman, editors. Quantitative Methods for Conservation Biology. Springerlink.

Pressey, R. L., C. J. Humphries, C. R. Margules, R. I. Vane-Wright, and P. H. Williams. 1993. Beyond opportunism key principles for systematic reserve selection. Trends in Ecology \& Evolution 8:124-128.

Qiao, H., J. Soberón, and T. A. Peterson. 2015. No silver bullets in correlative ecological niche modeling: Insights from testings among many potential algorithms for niche estimation. Methods in Ecology and Evolution.

R core team. 2014. R: A language and environment for statistical computing.in R. f. f. S. Computing, editor., Vienna, Austria.

Ratter, J. A., J. F. Ribeiro, and S. Bridgewater. 1997. The Brazilian Cerrado Vegetation and Threats to its Biodiversity. Annals of Botany 80:223-230.

Redford, K. H. and G. A. B. Fonseca. 1986. The role of Gallery Forests in the Zoogeography of the Cerrado' s non-volant mammalian fauna. Biotropica 18:126-135.

Reed, K. E. and J. G. Fleagle. 1995. Geographic and climatic control of primate diversity. Proceedings of the National Academy of Sciences 92:7874-7876.

Roberge, J.-M. and P. Angelstam. 2004. Usefulness of the Umbrella species concept as a conservation tool. Conservation Biology 18:76-85.

Rondinini, C., K. A. Wilson, L. Boitani, H. Grantham, and H. P. Possingham. 2006. Tradeoffs of different types of species occurrence data for use in systematic conservation planning. Ecology Letters 9:1136-1145.

Ronquist, F. 1997. Dispersal-Vicariance Analysis: a new approach to the quantification of Historical Biogeography. Systematic Biology 46:195-203.

Root, T. L., J. T. Price, K. R. Hall, H. Schneider, C. Rosenzweigk, and J. A. Pounds. 2003. Fingerprints of global warming on wild animals and plants. Nature 421:57-60.

Rothermel, B. B. and R. D. Semlitsch. 2002. An Experimental Investigation of Landscape Resistance of Forest versus Old-Field habitats to emigrating juvenile amphibians. Conservation Biology 16:1324-1332.

Ruiz-Miranda, C. R., A. G. Affonson, A. Martins, and B. Beck. 2000. Distribuição do sagui (Callithrix jacchus) nas áreas de ocorrência do mico leão dourado no 
(Leontopithecus rosalia) no estado do Rio de Janeiro. Neotropical Primates 8:98-101.

Russell, B. D., S. D. Connell, C. Mellin, B. W. Brook, O. W. Burnell, and D. A. Fordham. 2012. Predicting the distribution of commercially important invertebrate stocks under future climate. PLoS ONE 7:e46554.

Rylands, A. B. and R. A. Mittermeier. 2009. The Diversity of the New World Primates (Platyrrhini): An Annotated Taxonomy.in P. A. Garber, A. Estrada, J. C. BiccaMarques, E. W. Heymann, and K. B. Strier, editors. South American Primates: Comparative Perspectives in the Study of Behavior, Ecology, and Conservation. Springer New York.

Saccheri, I., M. Kuussaari, M. Kankare, P. Vikman, W. Fortelius, and I. Hanski. 1998. Inbreeding and extinction in a butterfly metapopulation. Nature 392:491-494.

Salazar, L. F., C. A. Nobre, and M. D. Oyama. 2007. Climate change consequences on the biome distribution in tropical South America. Geophysical Research Letters 34:n/a-n/a.

Salgado-Labouriau, M. L. 1994. História Ecológica da Terra. Editora Edgard Blucher Ltda., São Paulo - SP Brazil.

Salmona, Y. B. 2013. Cerrado com C ou com S? Modelagem de cenários futuros para o bioma. Dissertação. Universidade de Brasília, Brasília.

Sandel, B., L. Arge, B. Dalsgaard, R. G. Davies, K. J. Gaston, W. J. Sutherland, and J. C. Svenning. 2011. The influence of Late Quaternary climate-change velocity on species endemism. Science 334:660-664.

Santana, B. E. M. M., M. R. Prado, G. Lessa, E. C. Rocha, and F. R. Melo. 2008. Densidade, tamanho populacional e abundância dos primatas em MG. Revista Árvore 32:1109-1117.

São Bernardo, C. S. and M. Galetti. 2004. Densidade e tamanho populacional de primatas em um fragmento florestal no sudeste do Brasil. Revista Brasileira de Zoologia 21:827-832.

Scott, J. M., F. Davis, B. Csuti, R. Noss, B. Butterfield, H. Anderson, S. Caicco, F. D. Erchia, T. C. Edwards, J. Ulliman, H. A. L. Anderson, and R. G. Wright. 1993. Gap Analysis: a geographic approach to protection of biological diversity. Wildlife Monographs 123:3-41.

Sekercioglu, C. H., S. H. Schneider, J. P. Fay, and S. R. Loarie. 2008. Climate change, elevational range shifts, and bird extinctions. Conservation biology : the journal of the Society for Conservation Biology 22:140-150.

Silva, J. M. C. d. 1996. Distribution of amazonian and atlantic birds in gallery forests of the Cerrado region, South America. Ornitologia Neotropical 7:1-18.

Silva, J. M. C. d. 1997. Endemic bird species and conservation in the Cerrado Region, South America. Biodiversity and Conservation 6:435-450.

Silva, J. M. C. d. and J. M. Bates. 2002. Biogeographic patterns and conservation in the South American Cerrado: A tropical savanna hotspot. BioScience 52:225-234.

Silva, J. M. C. d. and D. C. Oren. 1996. Application of parsimony analysis of endemicity in Amazonian biogeography: an example with primates. Biological Journal of the Linnean Society 59:427-437.

Silva, J. M. C. d., M. C. d. Sousa, and C. H. M. Castelletti. 2004. Areas of endemism for passerine birds in the Atlantic forest, South America. Global Ecology and Biogeography 13:85-92.

Siqueira, M. F. d. and A. T. Peterson. 2003. Consequences of global climate change for geographic distributions of Cerrado tree species. Biota Neotropica 3:1-14. 
Soares-Filho, B., R. Rajão, M. Macedo, A. Carneiro, W. Costa, M. Coe, H. Rodrigues, and A. Alencar. 2014. Cracking Brazil's Forest Code. Science 344:363-364.

Soberón, J. 2007. Grinnellian and Eltonian niches and geographic distributions of species. Ecology Letters 10:1115-1123.

Soberón, J. and A. T. Peterson. 2005. Interpretation of models of fundamental ecological niches and species' distributional areas. Biodiversity Informatics 2:110.

Sóberon, J. and M. Nakamura. 2009. Niches and distributional areas: Concepts, methods, and assumptions. Proceedings of the National Academy of Sciences 106: $19644-19650$.

Stallings, J. R. 1985. Distribution and status of primates in Paraguay. Primate Conservation 6:51-57.

Svenning, J.-C., C. Fløjgaard, K. A. Marske, D. Nógues-Bravo, and S. Normand. 2011. Applications of species distribution modeling to paleobiology. Quaternary Science Reviews 30:2930-2947.

Swets, J. A. 1988. Measuring the accuracy of diagnostic systems. Science 240:12851293.

Szumik, C. and P. Goloboff. 2004. Areas of endemism: an improved optimality criterion. Systematic Biology 53:968-977.

Szumik, C. A., F. Cuezzo, P. A. Goloboff, and A. E. Chalup. 2002. An optimality criterion to determine areas of endemism. Systematic Biology 51:806-816.

Thomas, C. D., A. Cameron, R. E. Green, M. Bakkenes, L. J. Beaumont, Y. C. Collingham, B. F. N. Erasmus, M. F. d. Siqueira, A. Grainger, L. Hannah, L. Hughes, B. Huntley, A. S. v. Jaarsveld, G. F. Midgley, L. Miles, M. A. OrtegaHuerta, A. T. Peterson, O. L. Phillips, and S. E. Williams. 2004. Extinction risk from climate change. Nature 427:145-148.

Thorn, J. S., V. Nijman, D. Smith, and K. A. I. Nekaris. 2009. Ecological niche modelling as a technique for assessing threats and setting conservation priorities for Asian slow lorises (Primates:Nycticebus). Diversity and Distributions 15:289-298.

Thuiller, W. 2003. BIOMOD - optimizing predictions of species distributions and projecting potential future shifts under global change. Global Change Biology 9:1353-1362.

Thuiller, W. 2005. Climate change threats to plant diversity in Europe. Proceedings of the National Academy of Sciences 102:8245-8250.

Thuiller, W., O. Broennimann, G. Hughes, J. R. M. Alkemade, G. F. Midgley, and F. Corsi. 2006. Vulnerability of African mammals to anthropogenic climate change under conservative land transformation assumptions. Global Change Biology 12:424-440.

Thuiller, W., D. Georges, and R. Engler. 2014. biomod2: Ensemble platform for species distribution modeling. .

Thuiller, W., B. Lafourcade, R. Engler, and M. B. Araújo. 2009. BIOMOD - a platform for ensemble forecasting of species distributions. Ecography 32:369-373.

Turner, D. P., W. B. Cohen, R. E. Kennedy, K. S. Fassnacht, and J. M. Briggs. 1999. Relationships between Leaf Area Index and Landsat TM Spectral Vegetation Indices across Three Temperate Zone Sites. Remote Sensing of Environment 70:52-68.

UICN, WWF-Brasil, and IPÊ. 2011. Metas de Aichi: Situação atual no Brasil. Brasília, DF. 
Urban, M. C. 2015. Accelerating extinction risk from climate change. Science 348:571573.

Varela, S., R. P. Anderson, R. García-Valdés, and F. Fernández-González. 2014. Environmental filters reduce the effects of sampling bias and improve predictions of ecological niche models. Ecography:no-no.

Vasudev, D. and R. J. Fletcher. 2015. Incorporating movement behavior into conservation prioritization in fragmented landscapes: An example of western hoolock gibbons in Garo Hills, India. Biological Conservation 181:124-132.

Vilanova, R., J. d. S. e. S. Júnior, C. E. V. Grelle, G. Marroig, and R. CerqueirL. 2005. Limites climáticos e vegetacionais das distribuições de Cebus nigritus e Cebus robustus (Cebinae, Platyrrhini). Neotropical Primates 13:14-19.

Vivo, M. d. 1997. Mammalian evidence of historical ecological change in the Caatinga semiarid vegetation of Northeastern Brazil. Journal of Comparative Biology 2:65-73.

Waltari, E., R. J. Hijmans, A. T. Peterson, A. S. Nyári, S. L. Perkins, and R. P. Guralnick. 2007. Locating Pleistocene Refugia: comparing phylogeographic and Ecological Niche Model predictions. PLoS ONE 2:e563.

Wang, Q., S. Adiku, J. Tenhunen, and A. Granier. 2005. On the relationship of NDVI with leaf area index in a deciduous forest site. Remote Sensing of Environment 94:244-255.

Watson, J. E. M., H. S. Grantham, K. A. Wilson, and H. P. Possingham. 2011. Systematic Conservation Planning: Past, Present and Future.in R. J. Ladle and R. J. Whittaker, editors. Conservation Biogeography. Blackwell Publishing Ltd.

Werneck, F. P. 2011. The diversification of eastern South American open vegetation biomes: Historical biogeography and perspectives. Quaternary Science Reviews 30:1630-1648.

Werneck, F. P., G. C. Costa, G. R. Colli, D. E. Prado, and J. W. Sites Jr. 2011. Revisiting the historical distribution of Seasonally Dry Tropical Forests: new insights based on palaeodistribution modelling and palynological evidence. Global Ecology and Biogeography 20:272-288.

Werneck, F. P., C. Nogueira, G. R. Colli, J. W. Sites, and G. C. Costa. 2012. Climatic stability in the Brazilian Cerrado: implications for biogeographical connections of South American savannas, species richness and conservation in a biodiversity hotspot. Journal of Biogeography 39:1695-1706.

Willis, E. O. 1992. Zoogeographical origins of eastern Brazilian birds. Ornitologia Neotropical 3:1-15. 
CAPÍTUlO 5

Conclusões gerais 


\section{CONCLUSÕES}

O presente estudo analisou os padrões biogeográficos de primatas que ocorrem no Cerrado no passado, no presente e no futuro. Encontramos as seguintes evidências

- Durante os últimos 130 mil anos a fauna de primatas do Cerrado esteve associada, em sua maioria, a uma faixa ao sul do Cerrado que vai da Mata Atlântica ao Chaco;

- Existe uma congruência das distribuições das espécies que ocorrem nas transições no leste, no oeste e no norte, indicando que as espécies dessas áreas foram submetidas a forças que moldaram as suas distribuições tornando-as similares;

- Espécies do leste ocupam áreas de maior altitude, enquanto espécies do oeste e norte ocupam áreas de menor altitude;

- As características do meio que melhor explicam, em média, os padrões de distribuição das espécies são sazonalidade da temperatura e precipitação anual;

- As áreas prioritárias para conservação foram maiores e mais contínuas no norte do Cerrado e pequenas e isoladas no sul. Isso acontece por causa do alto índice de desmatamento ocorrido no sul do Cerrado;

- Quatro táxons estão previstos de serem possivelmente extintos do Cerrado nos próximos anos e mais três de ficarem com área bastante reduzida. Estes animais ocupam áreas na transição oeste e norte do Cerrado ou ocupam áreas da Caatinga;

- No futuro, a área de distribuição das espécies foi projetada de reduzir, em média, 9,3\% se as espécies puderem migrar livremente, 59,2\% se elas não puderem 
migrar e $84,4 \%$ se só puderem ocupar remanescentes nativos estimados para 2050

- O efeito do desmatamento foi maior do que o efeito das mudanças climáticas sobre a distribuição dos primatas do Cerrado.

As áreas prioritárias apontadas concordam, em parte, com as áreas de endemismo no sentido de que as maiores áreas estão dentro de áreas de endemismo e próximas da transição do bioma. Das quatro maiores áreas apontadas como prioritárias para conservação a do Tocantins e Piauí encontra-se marginalmente nas áreas de endemismo Transição Leste e Serras do Centro Leste e a do Rio Araguaia tem ocorrência marginal em todas as áreas de endemismo. Já a área do oeste do Mato Grosso se encontra no núcleo da área de endemismo Transição Oeste e a área em Minas Gerais se encontra no núcleo das áreas de endemismo Transição Leste e Serras do Centro Leste. Estas duas áreas prioritárias (em Mato Grosso e em Minas Gerais) são particularmente importantes para a conservação, pois apresentam alta riqueza de primatas, se encontram em áreas de endemismo e estão em locais mais ameaçados pelo avanço do desmatamento.

Por sua vez, os quatro táxons que estão previstos de serem extintos do Cerrado no futuro ocorrem no norte e oeste do domínio e estão mais associados às áreas Transição Norte e Transição Oeste. Para se conservar áreas prioritárias que protejam esses quatro táxons as mais indicadas são as áreas mais ao oeste, no Rio Araguaia e oeste do Mato Grosso. O Sapajus xanthosternos não está presente em nenhuma das quatro maiores áreas prioritárias apontadas no capítulo 2 .

Existe uma tendência das espécies procurarem áreas mais frias no futuro devido ao aquecimento global, se deslocando para áreas de alta altitude ou alta latitude (ver capítulo 4). Isso pode explicar porque três das quatro espécies mais afetadas no Cerrado serem as representantes das áreas Transição Oeste e Norte que possuem associação com 
áreas de baixa altitude. Outras espécies que possivelmente sofrerão mais com as mudanças globais são as que ocorrem no Cerrado e na Caatinga, porque estão em regiões de baixa latitude e que já apresenta temperaturas elevadas e baixa precipitação em boa parte do ano.

\section{Limitações do estudo}

O presente estudo utilizou de muitas técnicas e modelos ecológicos e cada um deles possui um erro associado. Os algoritmos de modelagem de nicho ecológico são estimativas do nicho abiótico (grinneliano) e não levam em consideração áreas que estão inacessíveis para as espécies ou áreas em que existe uma espécie competidora ou predadora no local (Soberón 2007, Peterson et al. 2011). Se houver uma grande área inacessível ou muita interação biótica os modelos se tornam mais imprecisos. Quando juntamos também as predições de vários algoritmos em um único mapa de consenso procuramos reduzir incertezas, porém adicionamos mais uma possível fonte de erro já que cada algoritmo faz uma previsão de um modo diferente e podem ter resultados bem diferentes (Buisson et al. 2010). Por causa disso decidimos usar somente modelos com um valor alto na medida de avaliação (AUC).

As variáveis ambientais utilizadas como input nos modelos também é uma fonte de erro. Essas variáveis são obtidas a partir de estações meteorológicas espalhadas ao redor do mundo, no entanto a concentração de estações meteorológicas no mundo não é uniforme e algumas regiões apresenta menor densidade de pontos de informações ambientais. Devido a isso, as variáveis ambientais tem diferentes níveis de extrapolação ao longo do mapa. Além disso, as variáveis projetadas para o passado e para o futuro são obtidas a partir de simulação de correntes oceânicas e atmosféricas e também com 
pontos de coleta de amostras de gelo que informam o clima do passado. Cada uma dessas projeções das variáveis ambientais possui também um erro associado já que são modelos matemáticos.

A medida de avaliação do modelo utilizada foi o AUC, que é a medida implantada no software utilizado e é também a mais utilizada nos últimos anos. No entanto, essa medida sofre muitas críticas de alguns autores que afirmam que é uma medida que superestima modelos de alguns algoritmos em relação a outros e também trata a curva ROC de uma forma inapropriada (Lobo et al. 2008). A forma de avaliação mais recomendada por especialistas é o uso de dados independentes para se fazer a avaliação, vindos de outra base de dados (geralmente vindos de trabalhos de campo). No entanto, isso é uma recomendação que na maioria dos casos é de difícil implementação pela dificuldade de tempo e de dinheiro para se obter duas bases de dados independentes para cada táxon.

A estimativa de desmatamento feita para o ano de 2050 possui também um erro associado, já que são definidos alguns pressupostos que podem não se concretizar (por exemplo, que a taxa de desmatamento permanecerá igual à existente entre 2002 e 2008). Todo programa computacional e estimativa estatística realizada também possuem seu erro associado, como ocorre com: o programa para priorização de áreas (Maxan), o modelo de efeito misto e o software para delimitação de áreas de endemismo (NDM/VNDM).

Os resultados deste trabalho devem ser interpretados com cautela, pois todos os modelos são simplificações da realidade. No entanto, utilizamos os métodos mais avançados na atualidade para gerar as predições mais robustas possíveis. Estas predições auxiliam o entendimento das modificações nos padrões biogeográficos e também no planejamento para a conservação. 


\section{Implicações para conservação}

Em termos práticos, visando à implementação de medidas que melhorem o grau de conservação dos primatas no Cerrado, podemos considerar algumas ações possíveis. Primeiramente, as áreas apontadas como prioritárias para a conservação dos primatas são áreas importantes para se considerar na hora de decidir por novas unidades de conservação. Como preferência deve-se focar em criar reservas na área maior que fica em Minas Gerais e em seguida na que fica no oeste de Mato Grosso, pois elas estão presentes em regiões com alta riqueza de primatas (fig. 3 do capítulo 2) e dentro de núcleos de áreas de endemismo (duas áreas de endemismo em MG e uma em MT, fig. 6 do capítulo 3). A área em Mato Grosso também é importante porque abriga o Mico melanurus, animal que está previsto de perder área em toda a sua distribuição e pode se extinguir totalmente por causa das mudanças climáticas (tabela 2 do capítulo 4).

Alguns táxons apresentaram projeções de possível perda de área que põe em risco a sobrevivência deles, dentro e fora do Cerrado. Para esses táxons é recomendável implantar programas de monitoramento populacional para acompanhar as variações na população e poder agir de forma mais rápida, caso seja efetivamente necessário. Três táxons apresentam perda de área elevada em toda a sua extensão geográfica: Mico melanurus, Aotus azarae infulatus e Sapajus libidinosus. Outros dois podem se extinguir somente do Cerrado: Alouatta belzebul e Sapajus xanthosternos.

Além disso, é necessário atuar no sentido de reduzir os fatores que estão causando maior impacto nas populações de primatas ameaçados: desmatamento e caça (Machado et al. 2008). O desmatamento possivelmente terá um impacto no futuro ainda maior do que o impacto das mudanças climáticas nas populações de primatas. A taxa de 
desmatamento do Cerrado nos últimos anos foi muito elevada e se o ritmo continuar assim é possível que todo o bioma esteja ameaçado e venha a desaparecer totalmente, como previsto em outro trabalho (Machado et al. 2004). É necessário um esforço dos governos e dos fazendeiros para reduzir o desmatamento e é incentivado projetos de reflorestamento com árvores nativas do Cerrado.

A caça de animais silvestres é uma ameaça para alguns primatas, que são vistos pelas populações humanas como atraentes e são capturados da natureza para serem vendidos como animais de estimação. Apesar de proibido, ainda existe tráfico de animais silvestres no país e é necessário aumentar a fiscalização em cima dessa prática para que se reduza o risco de extinção das espécies mais procuradas.

Ainda se todas as medidas propostas forem implementadas é incerto se os efeitos decorrentes serão suficientes para manter esses animais fora da lista de ameaçados. Um maior número de estudos nesse campo do conhecimento que modifique os pressupostos assumidos neste trabalho para testar novas interpretações ou que foque em outros táxons ou regiões é importante para analisarmos a real probabilidade de extinção da primatofauna e a melhor forma de se planejar para evitá-la.

\section{REFERÊNCIAS BIBLIOGRÁFICAS}

Buisson, L., W. Thuiller, N. Casajus, S. Lek, and G. Grenouillet. 2010. Uncertainty in ensemble forecasting of species distribution. Global Change Biology 16:1145-1157.

Lobo, J. M., A. Jiménez-Valverde, and R. Real. 2008. AUC: a misleading measure of the performance of predictive distribution models. Global Ecology and Biogeography 17:145-151.

Machado, A. B. M., G. M. Drummond, and A. P. Paglia. 2008. Livro Vermelho da Fauna Brasileira ameaçada de extinção, volume 2. MMA, Brasília-DF.

Machado, R. B., M. B. R. Neto, P. G. P. Pereira, E. F. Caldas, D. A. Gonçalves, N. S. Santos, K. Tabor, and M. Steininger. 2004. Estimativas de perda da área do Cerrado brasileiro. Brasília-DF.

Peterson, A. T., J. Soberón, R. G. Pearson, R. P. Anderson, E. Martinez-Meyer, M. Nakamura, and M. B. Araujo. 2011. Ecological Niches and Geographic Distributions. Princeton University Press, Princeton, USA.

Soberón, J. 2007. Grinnellian and Eltonian niches and geographic distributions of species. Ecology Letters 10:1115-1123. 
ANEXOS 


\section{Anexo 1 - Diferenças entre pares de projeções para o passado}
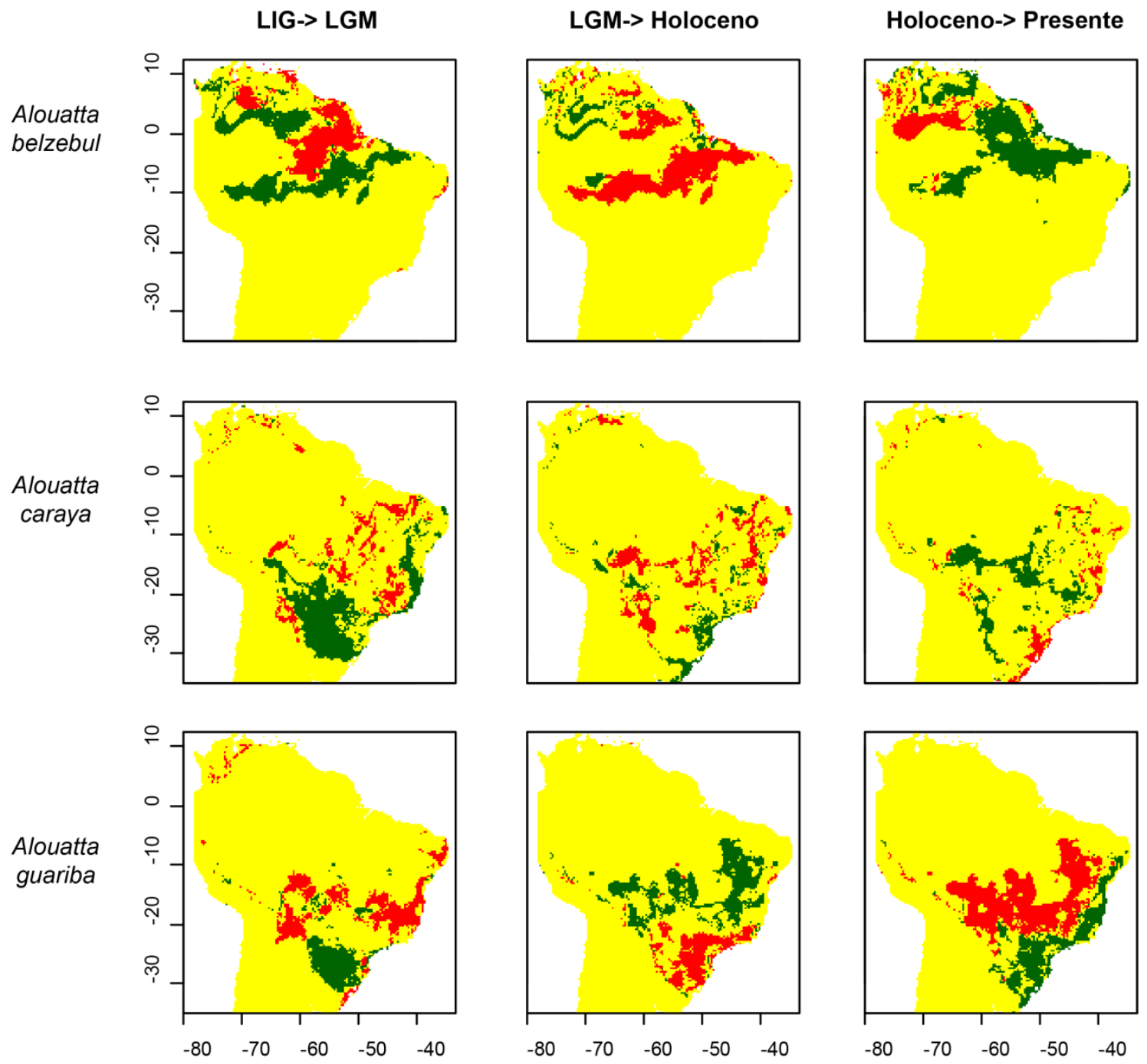

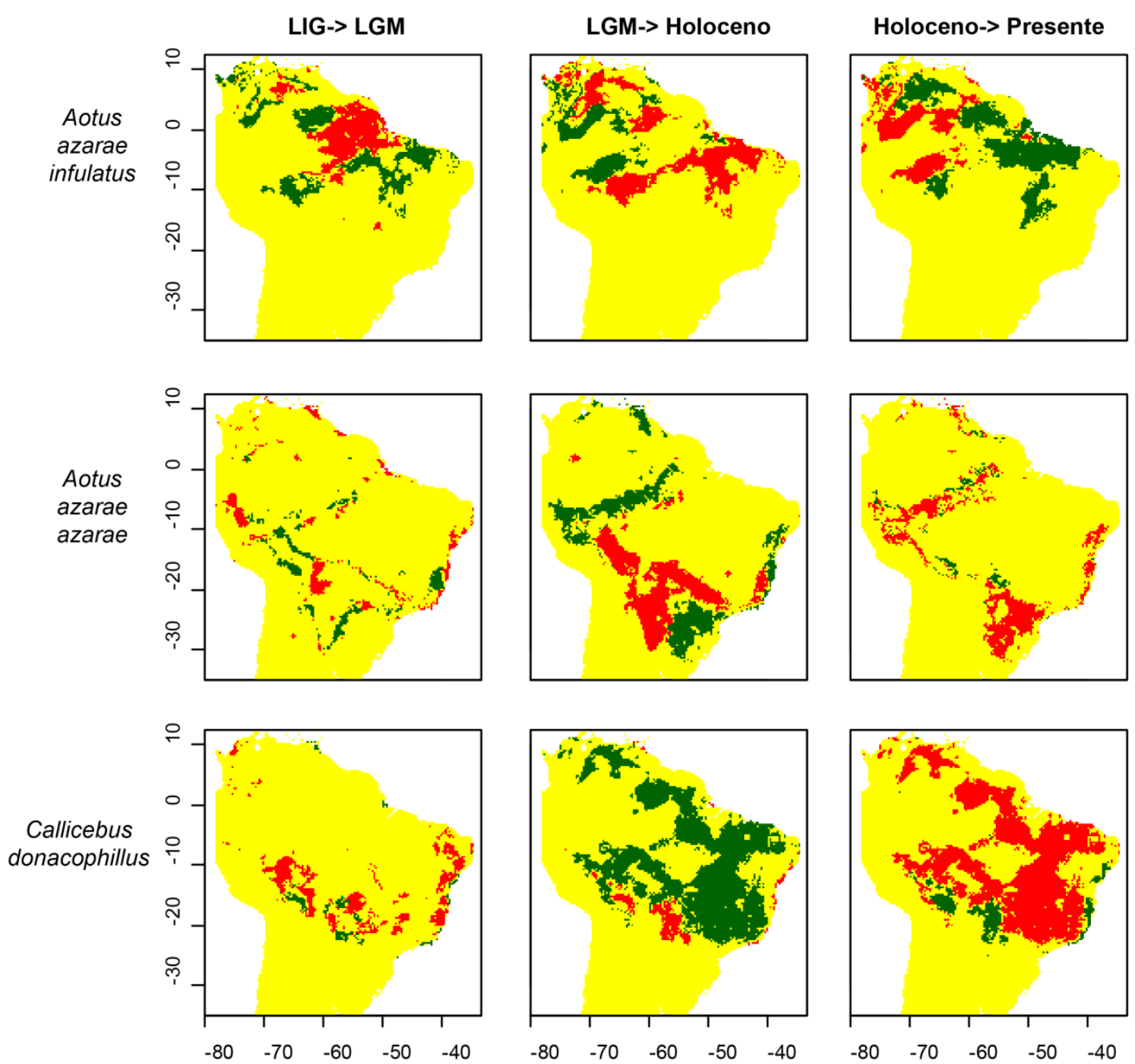

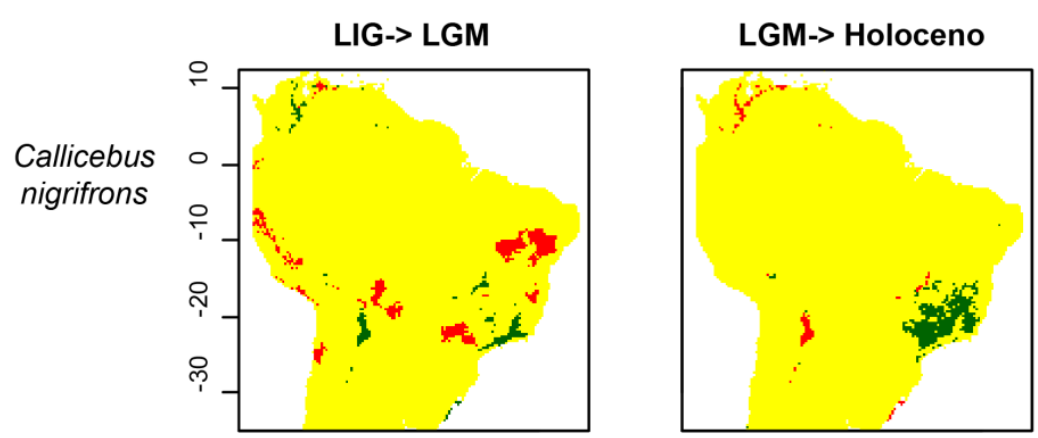

Holoceno-> Presente
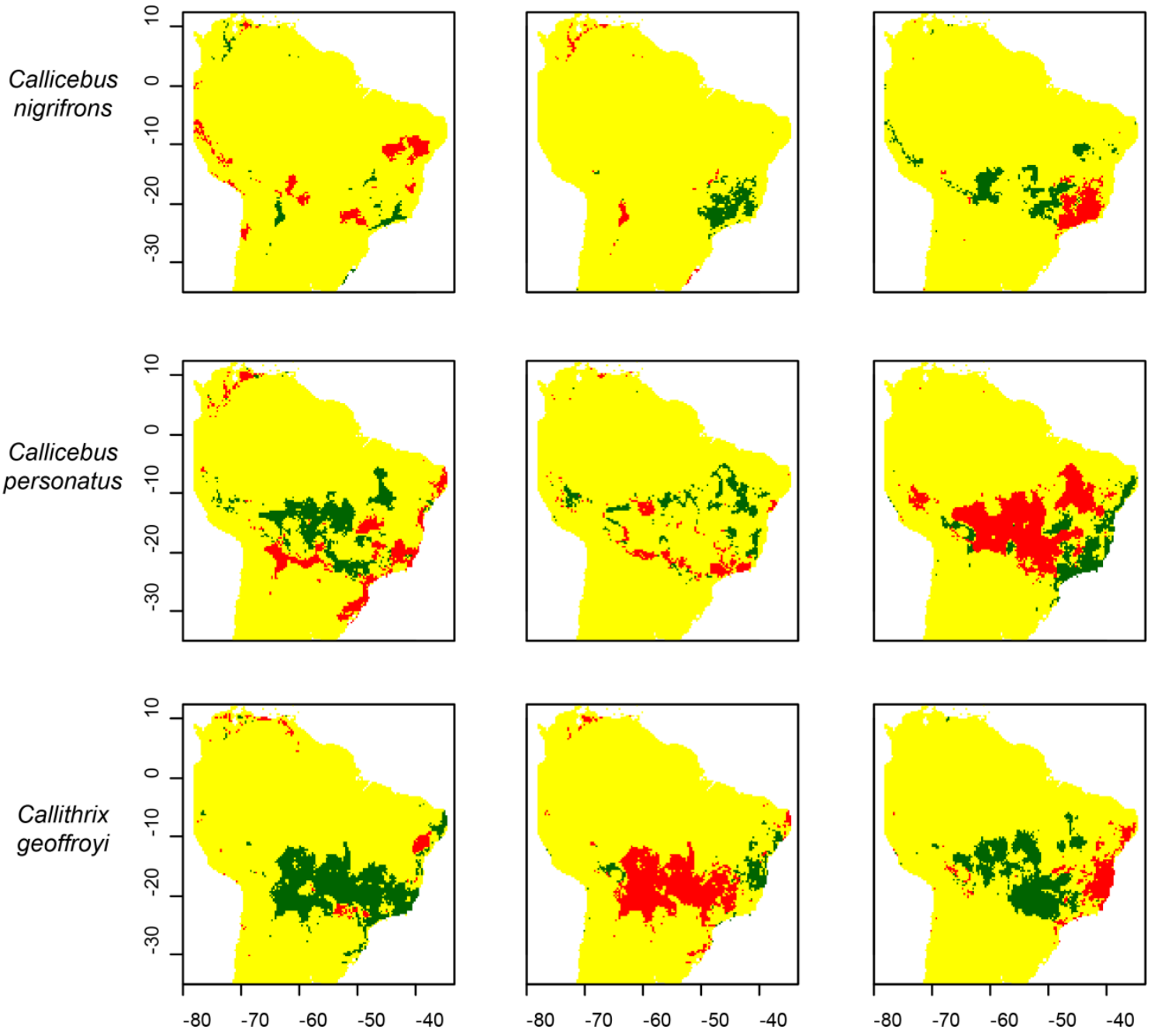


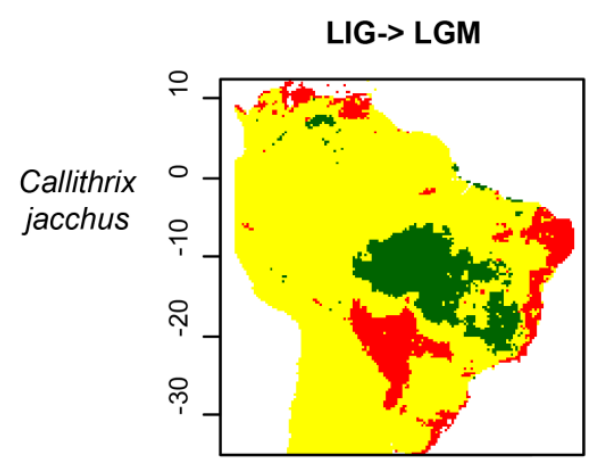

LGM-> Holoceno
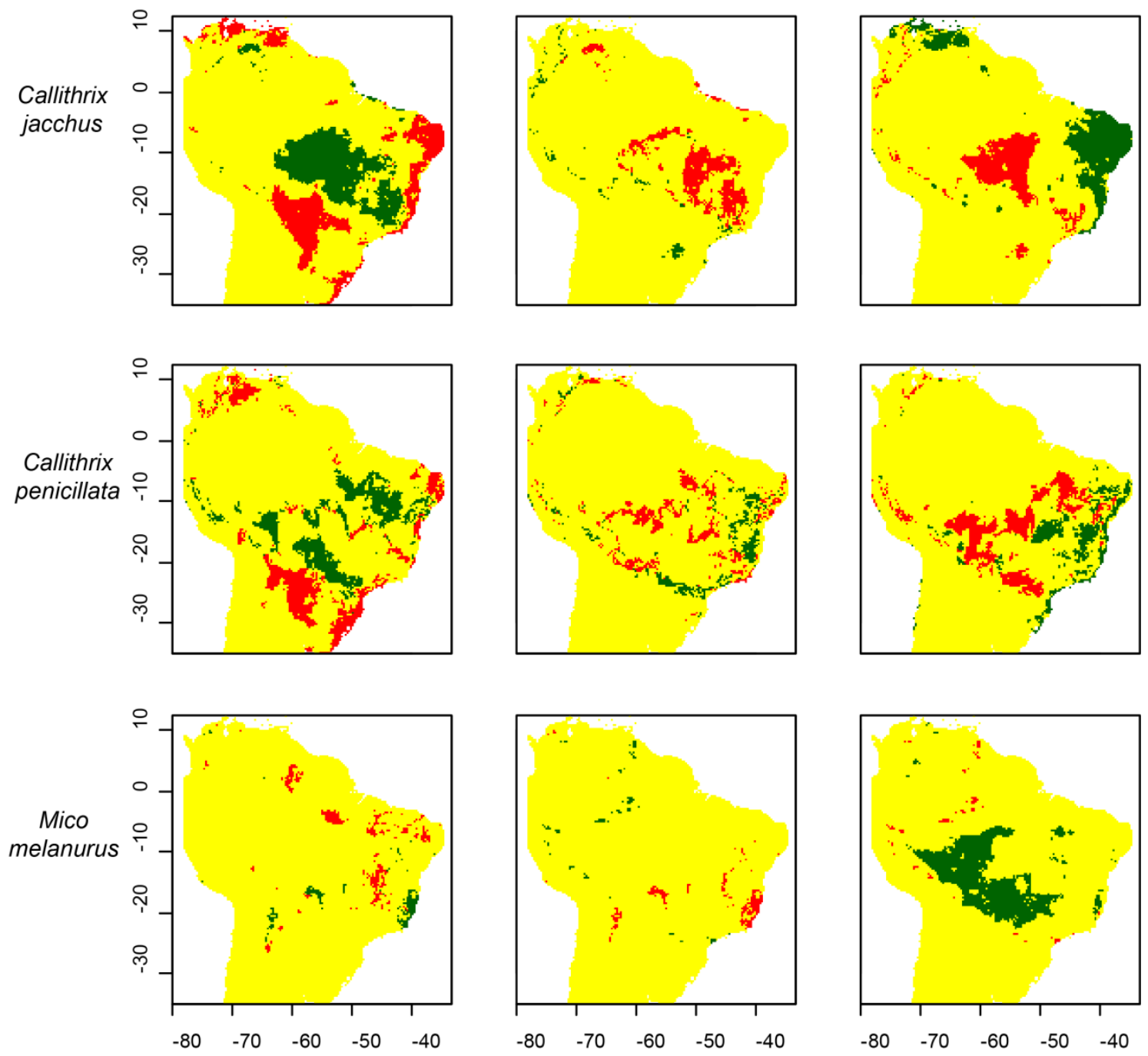

Holoceno-> Presente
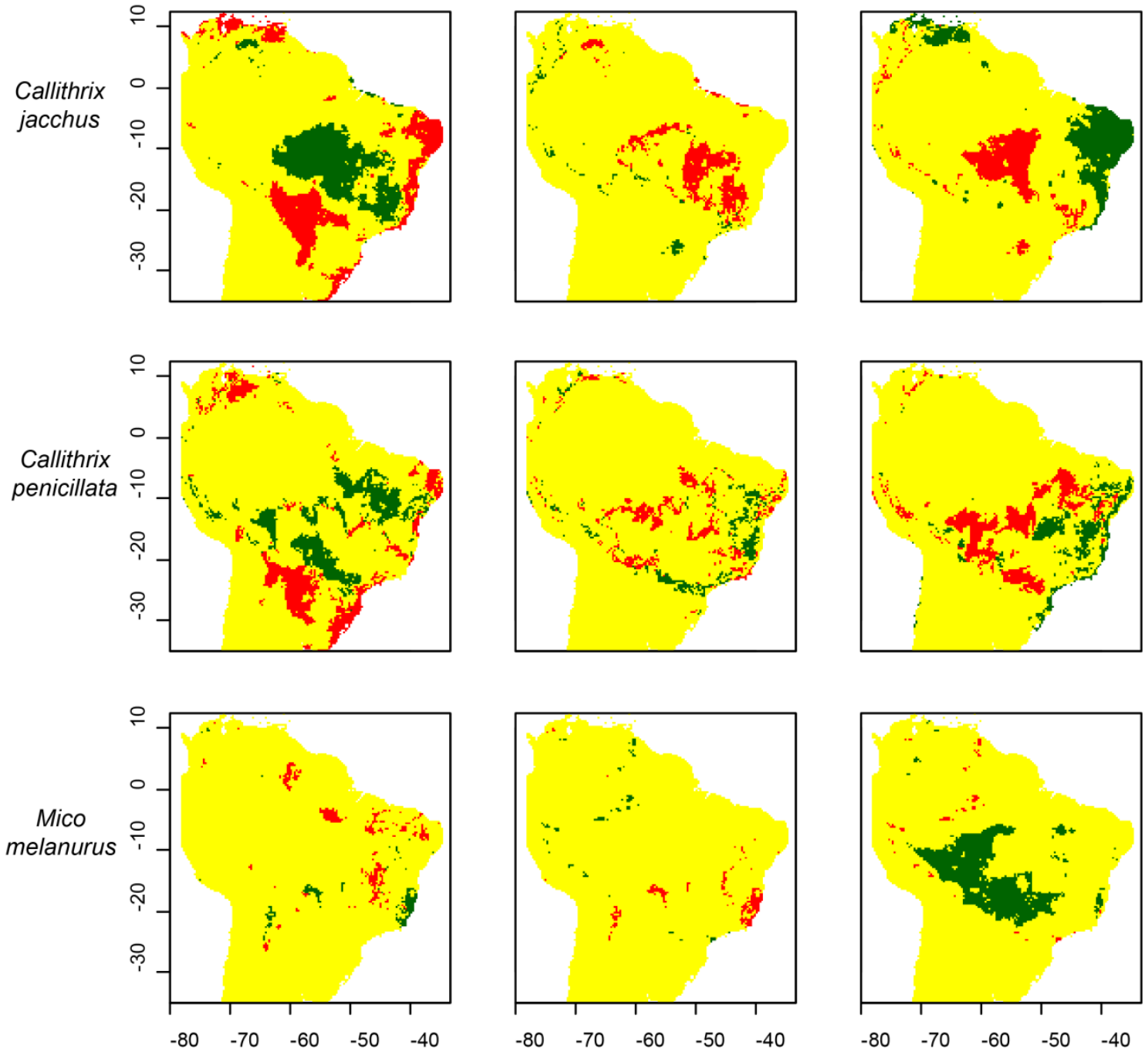

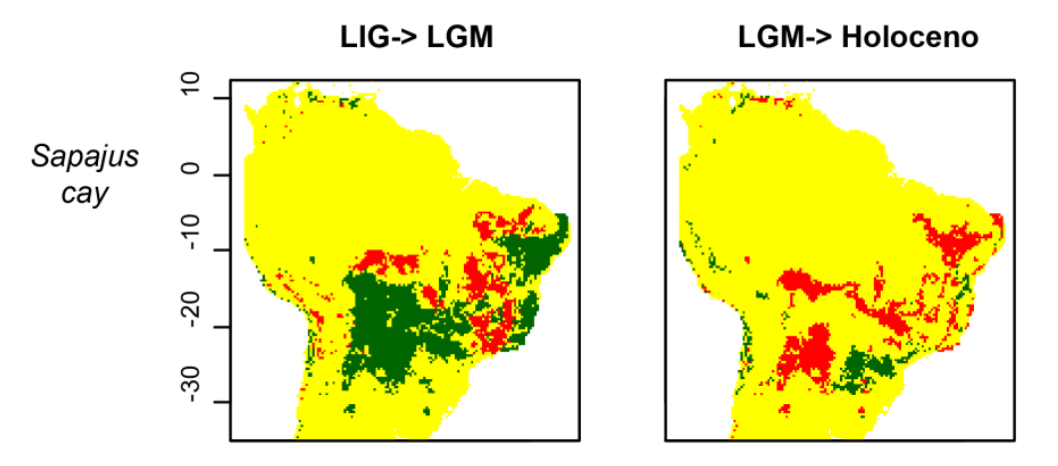

Holoceno-> Presente
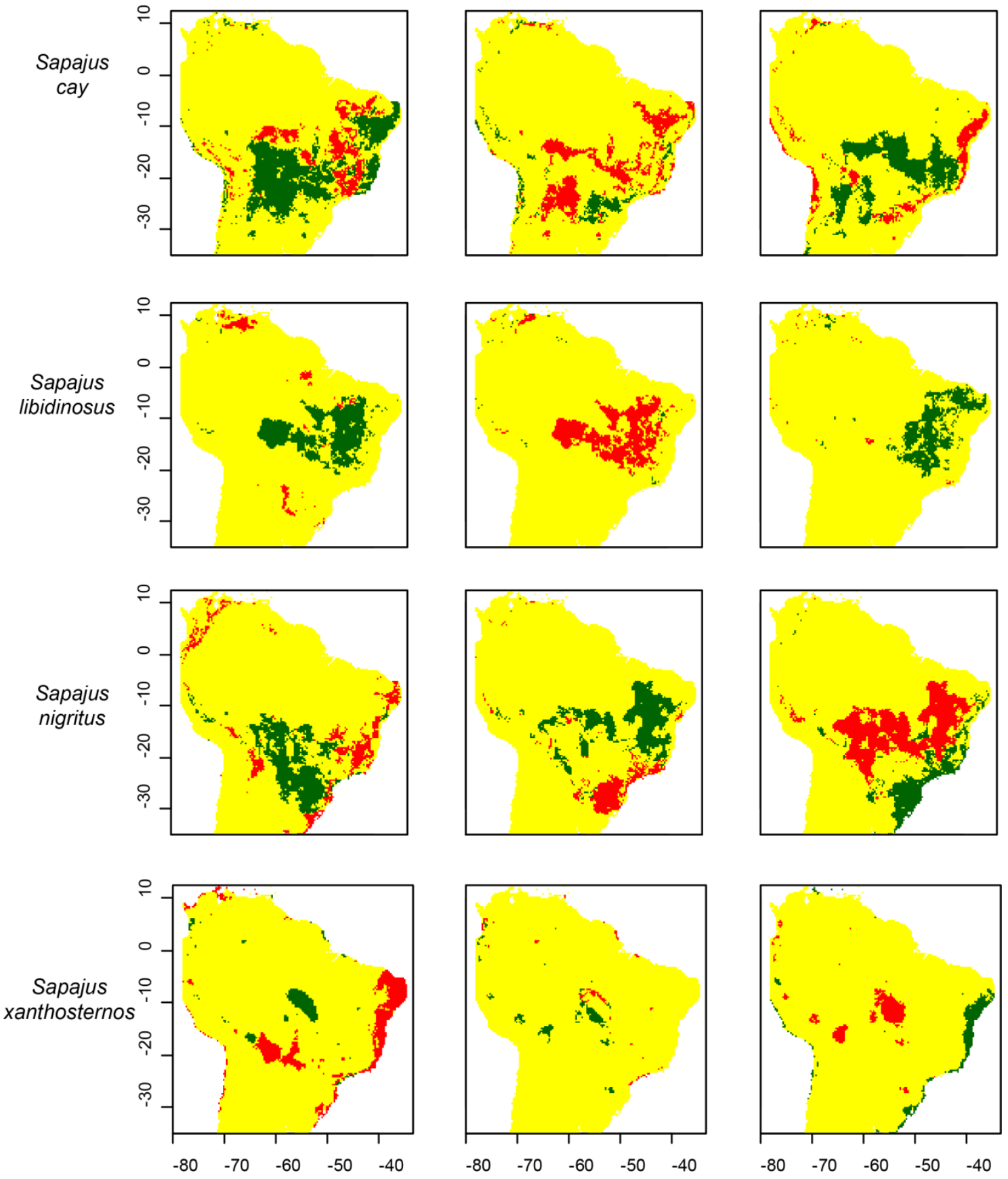

Figura A.1. Figuras ilustrando as diferenças entre pares de projeções temporais para o passado. Áreas em verde representam áreas que aumentaram significativamente a sua adequabilidade ambiental no período mais recente, áreas em vermelho representam áreas que diminuíram significativamente a adequabilidade ambiental e áreas em amarelo não modificaram seu valor entre os períodos de tempo. 


\section{Anexo 2- Remanescentes nativos no Cerrado para o presente e estimados para 2050}
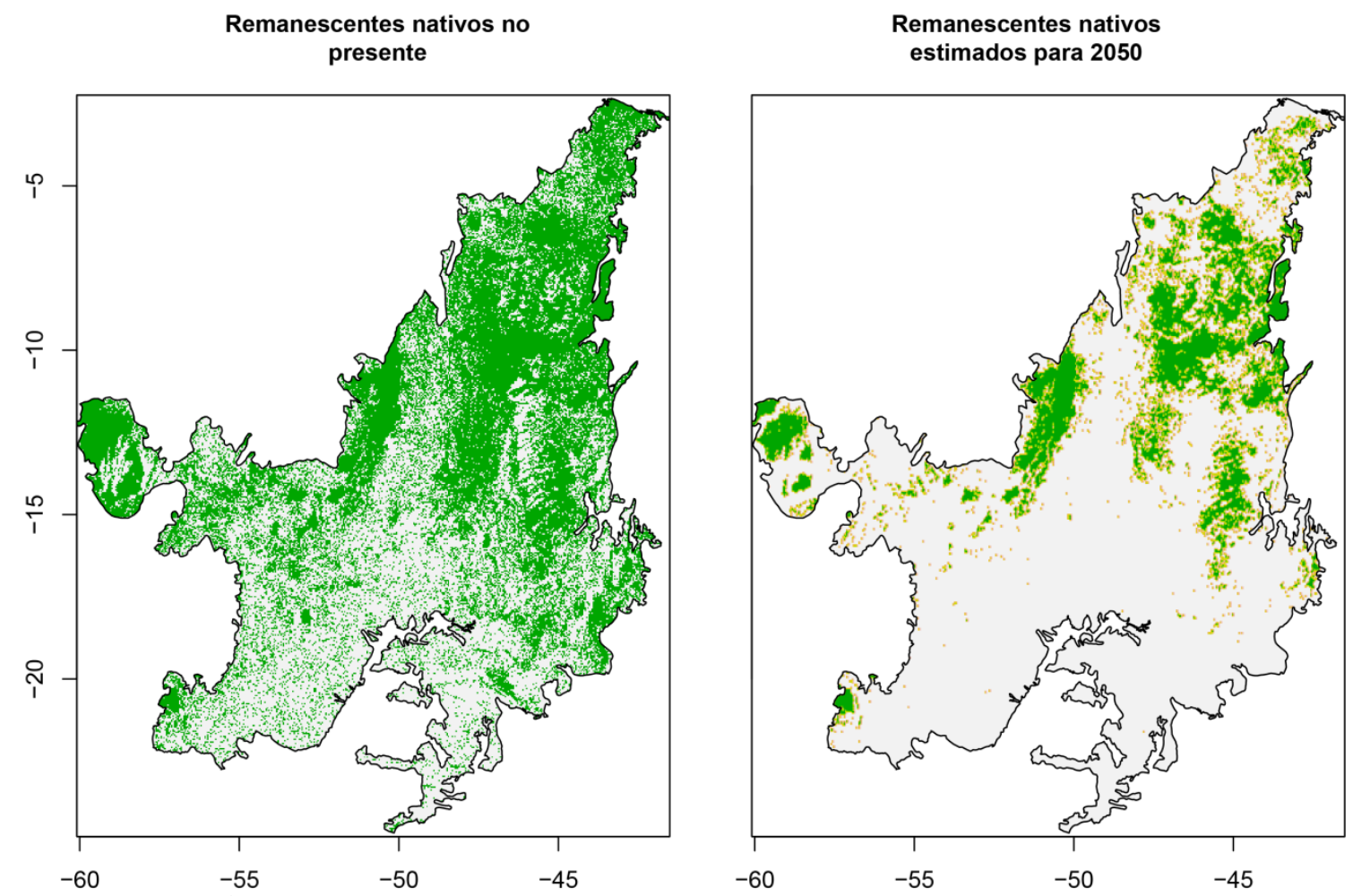

Figura A.2. Áreas de remanescentes nativos no Cerrado (em verde) no presente e estimados para o ano de 2050 (MMA/IBAMA, 2011; Faleiro et. al., 2013).

Faleiro, F. V., R. B. Machado, and R. D. Loyola. 2013. Defining spatial conservation priorities in the face of land-use and climate change. Biological Conservation 158:248-257.

MMA/IBAMA. (2011b). Monitoramento do desmatamento nos biomas brasileiros por satélite: acordo de cooperação técnica MMA/IBAMA. Monitoramento do bioma Cerrado 2009-2010. In. Brasília-DF: MMA. 
ANEXO 3 - PROJEÇÕES PARA O FUTURO NOS QUATRO DIFERENTES MODELOS GLOBAIS DE CIRCULAÇÃO ATMOSFERA-OCEANO E CENÁRIOS CLIMÁTICOS

Alouatta belzebul
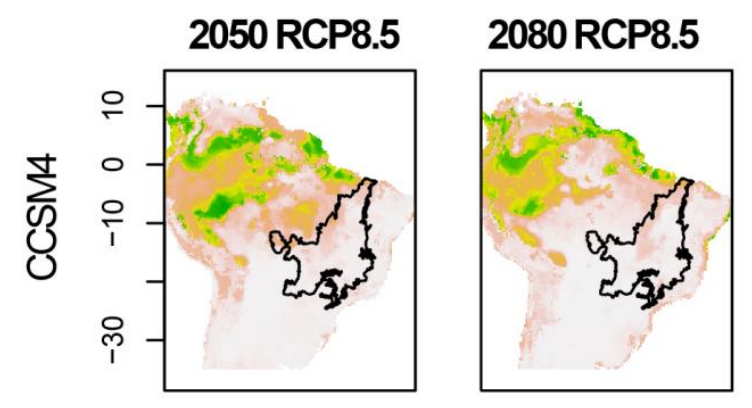

2050 RCP2.6
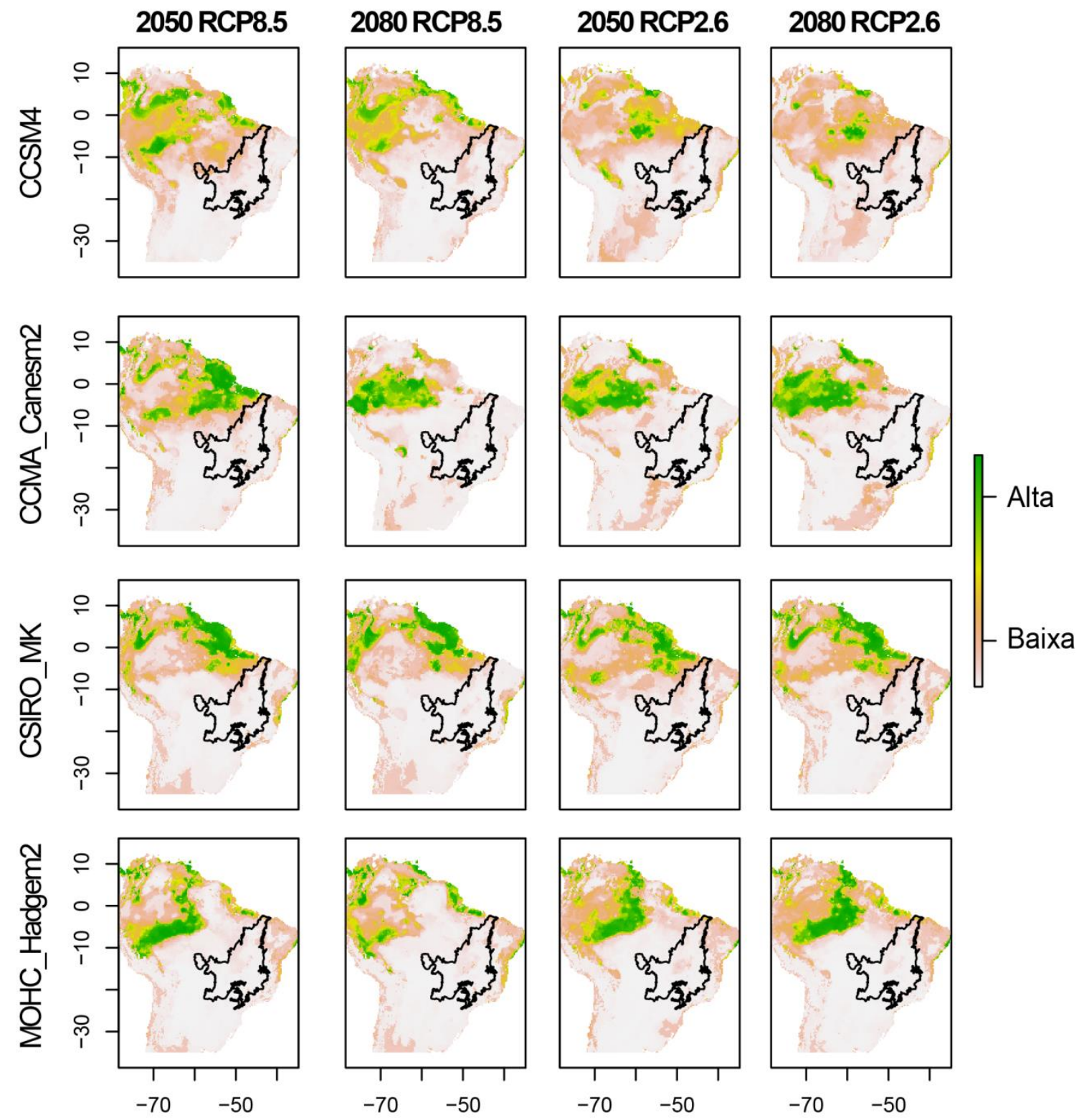
Alouatta caraya
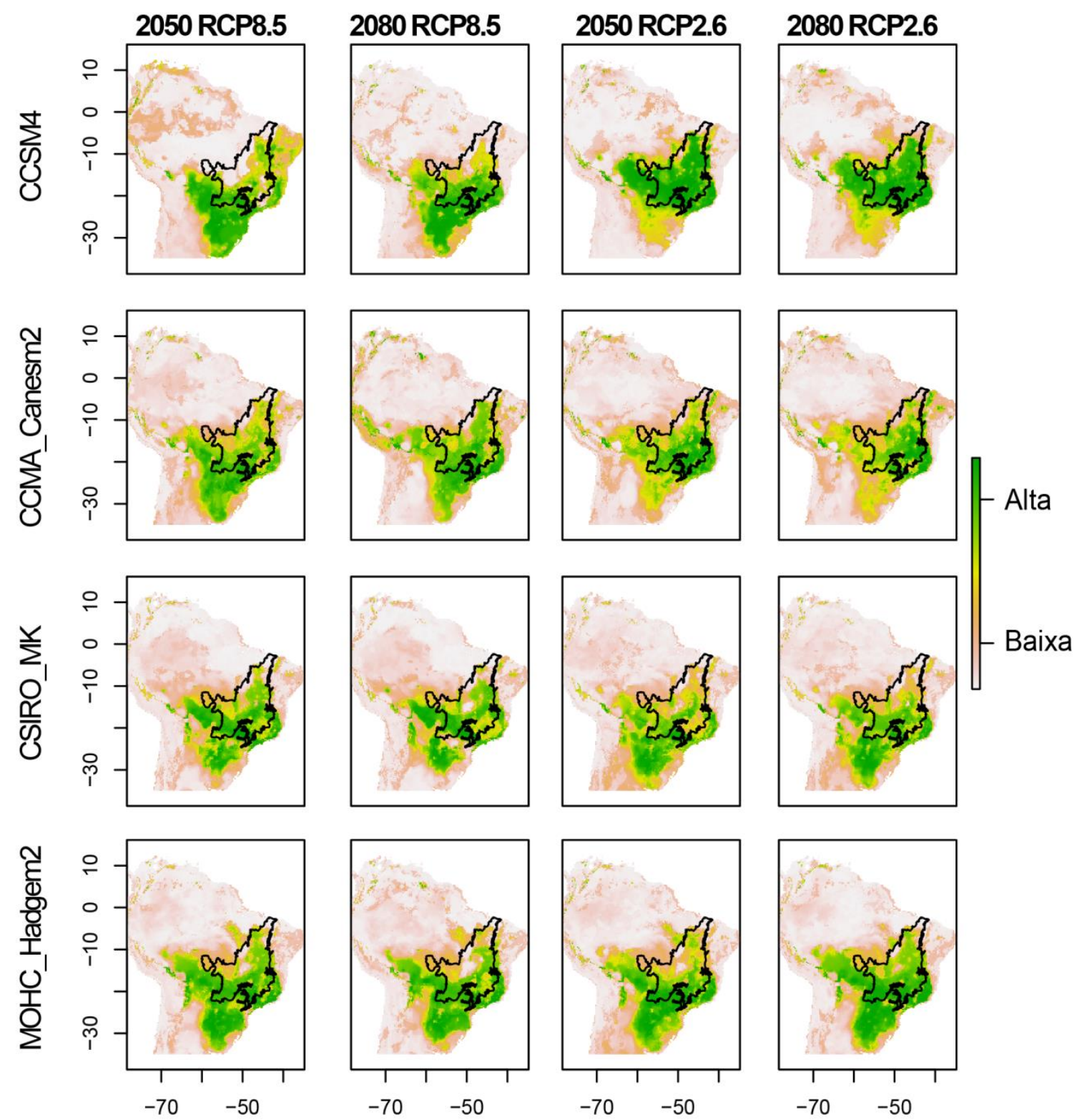
Alouatta guariba
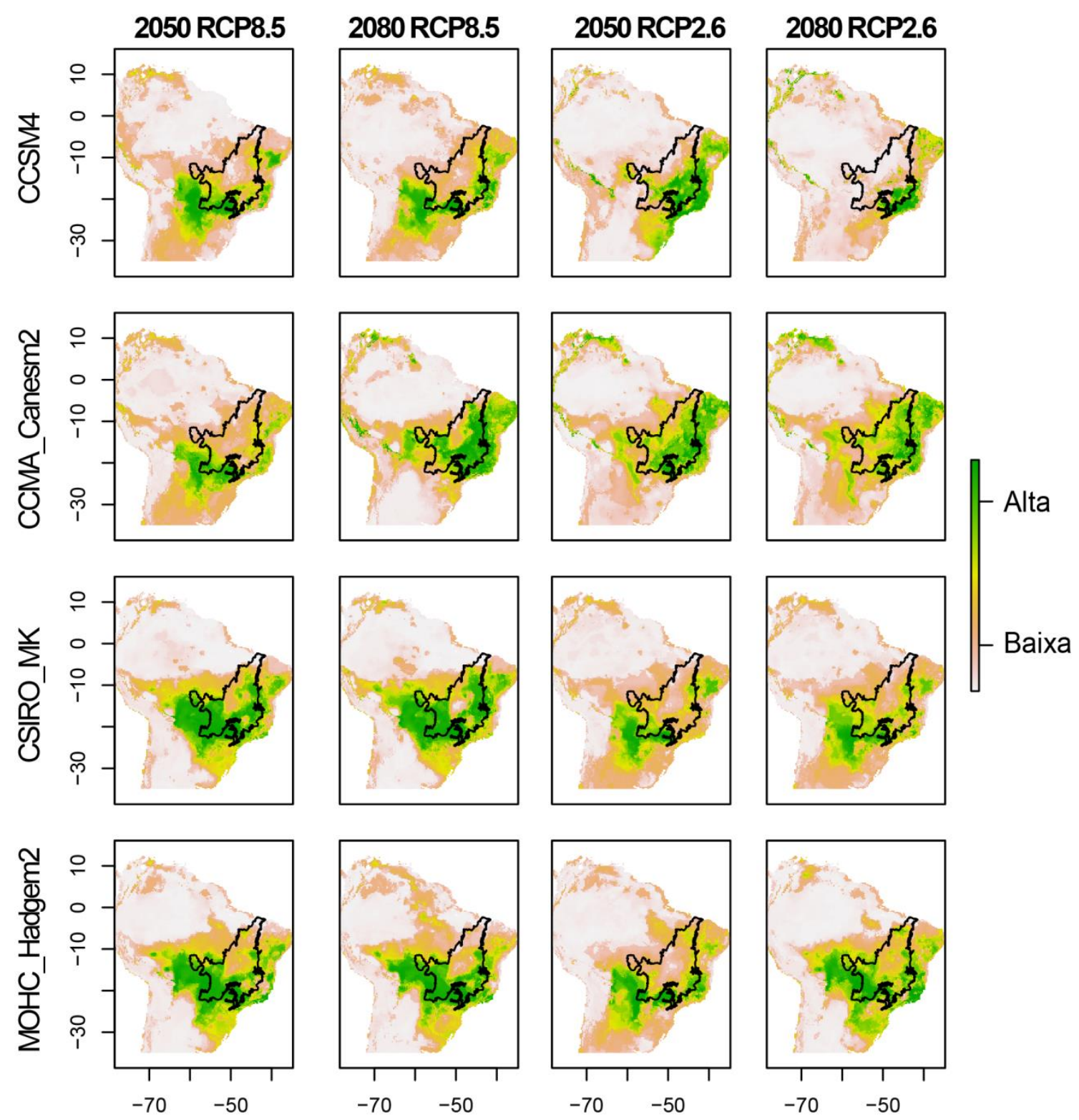
Aotus azarae azarae
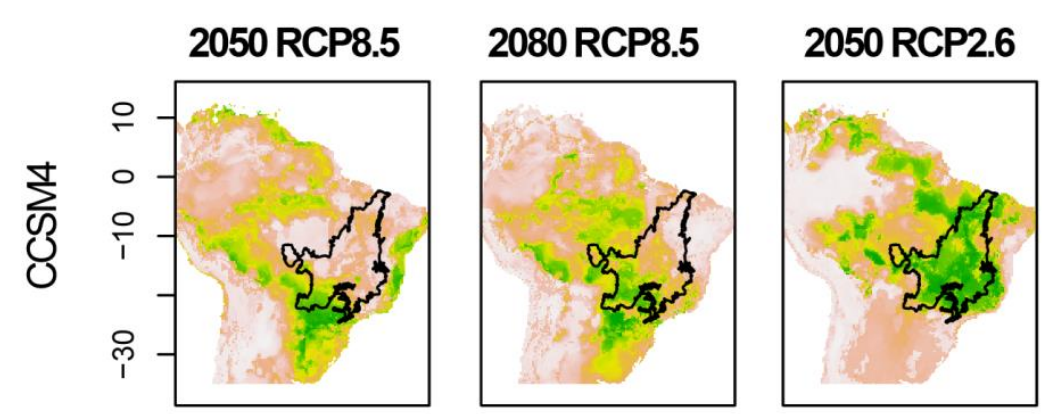

2080 RCP2.6
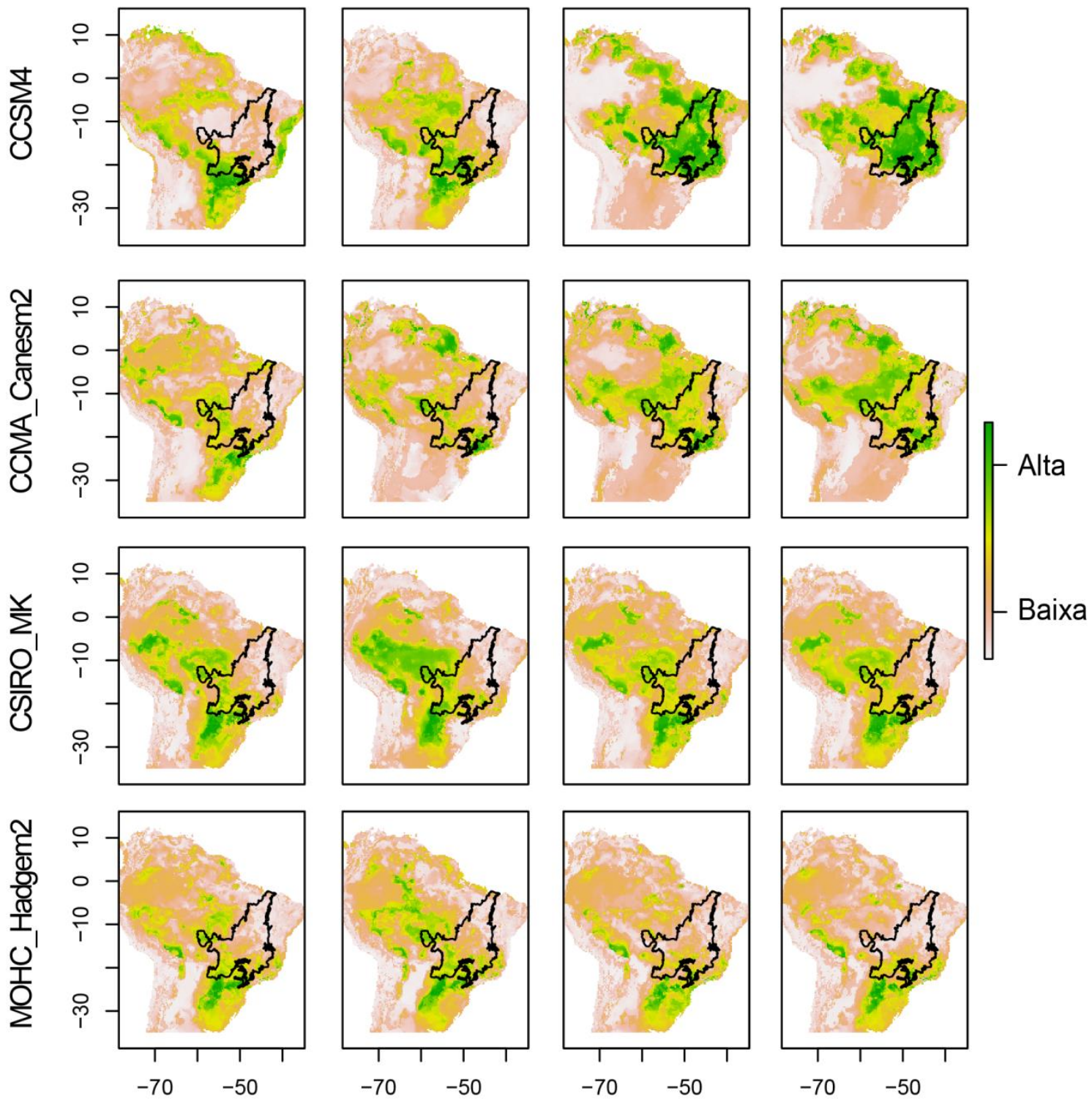
Aotus azarae infulatus
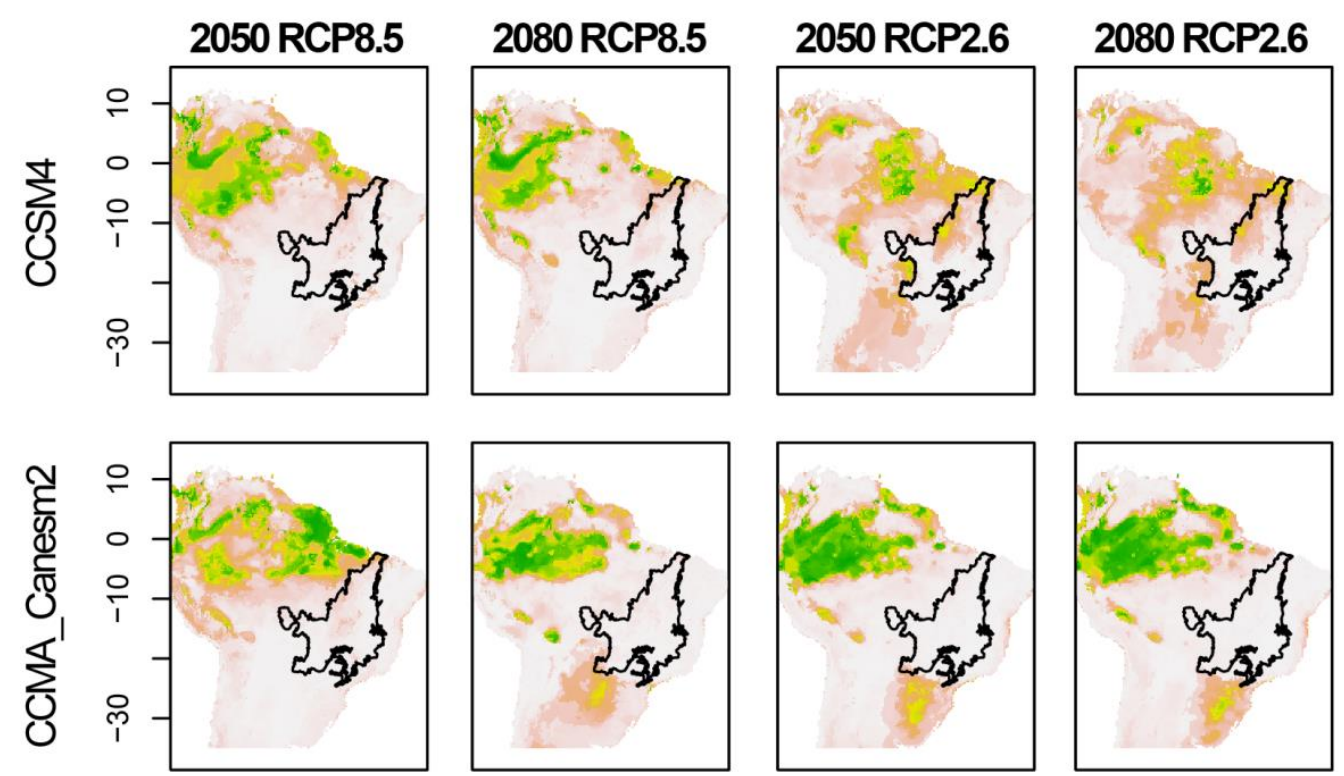

- Alta
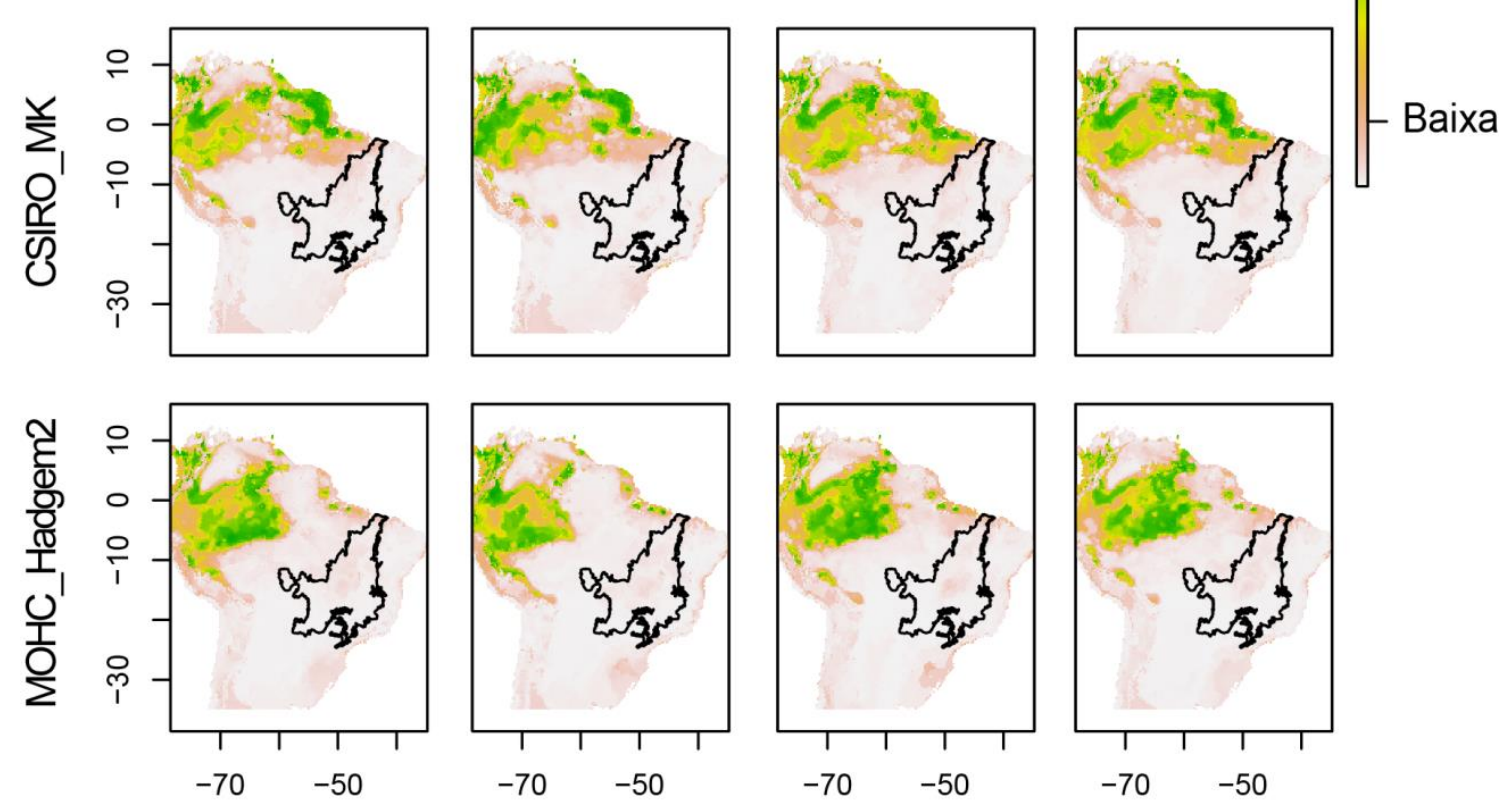
Callicebus donacophilus
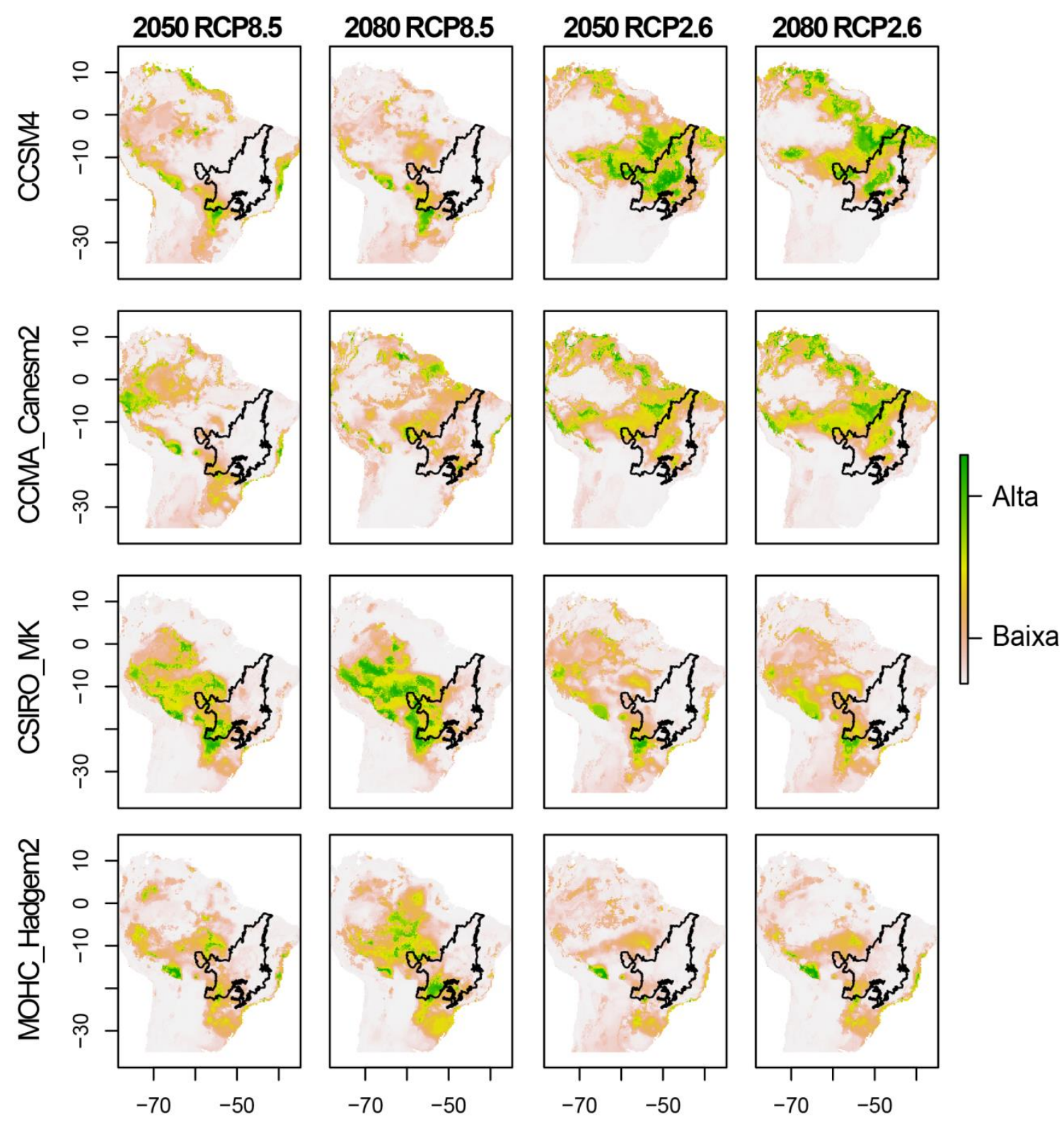
Callicebus nigrifrons
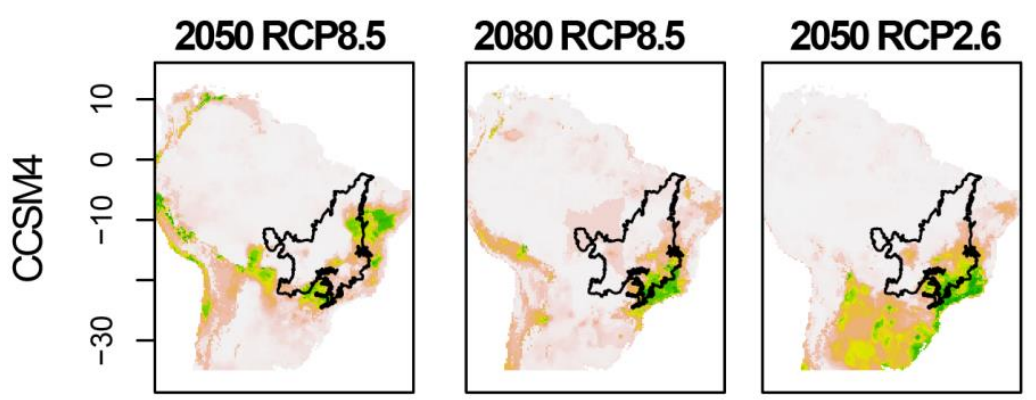

2080 RCP2.6
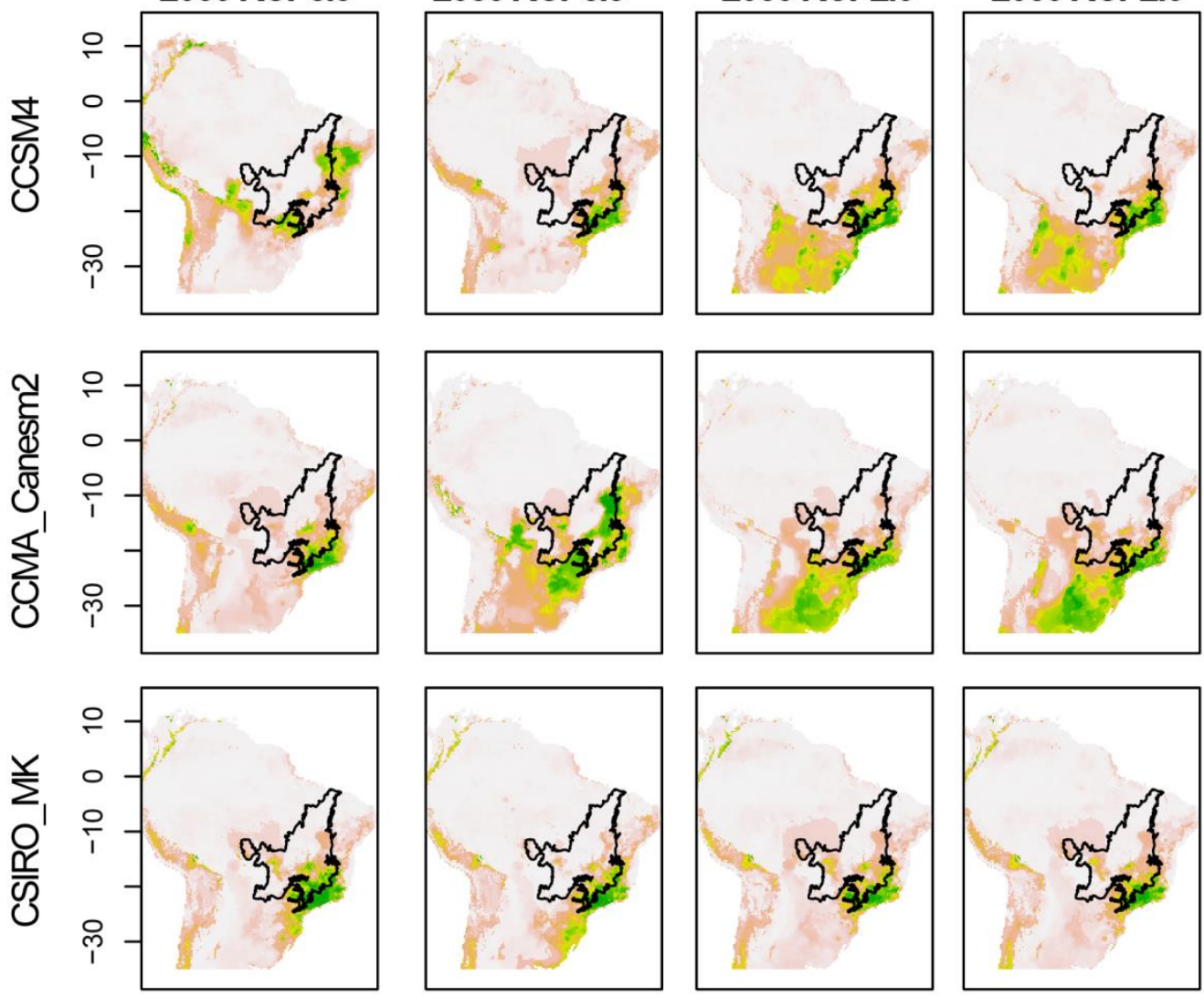

Alta
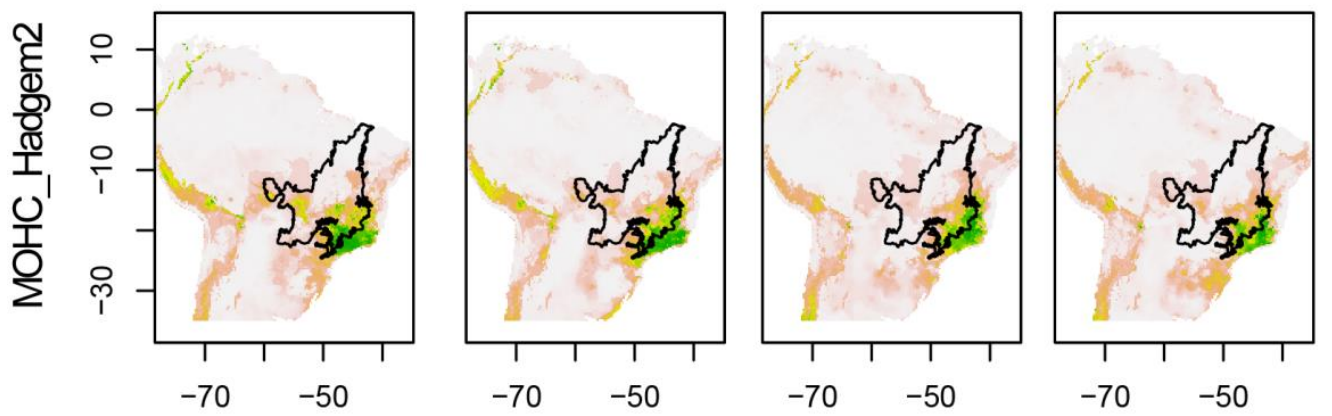
Callicebus personatus
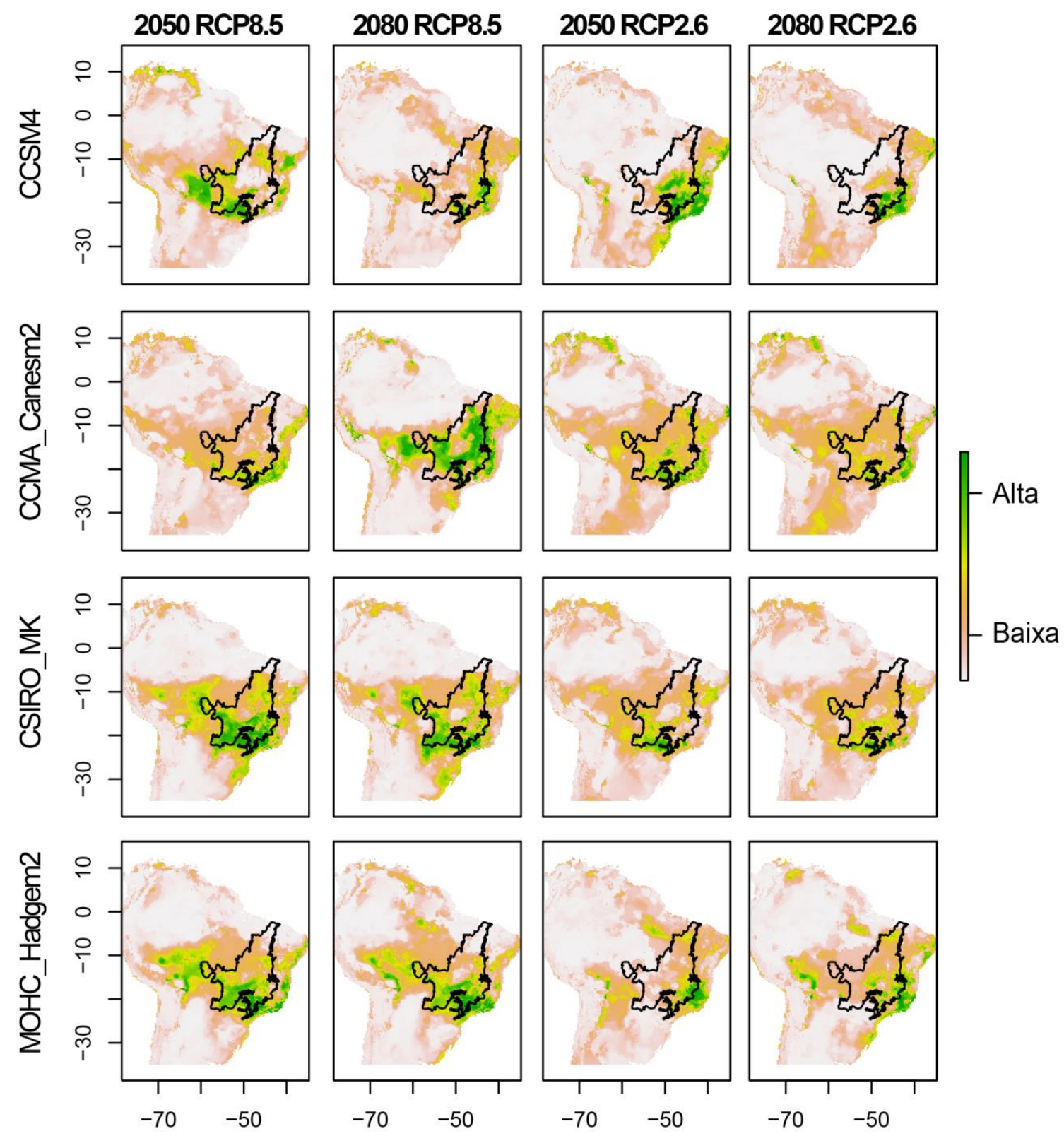
Callithrix geoffroyi
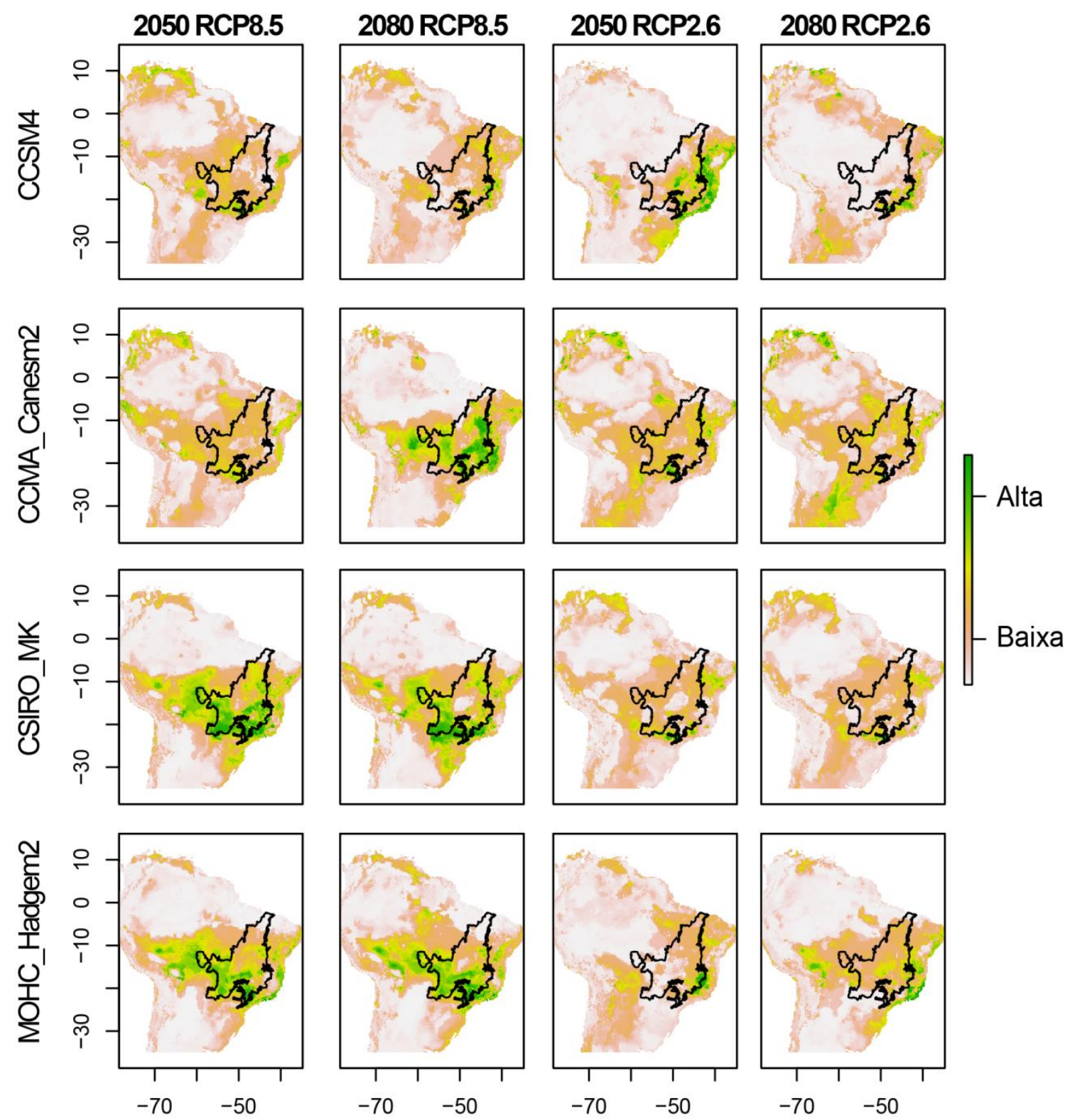


\section{Callithrix jacchus}
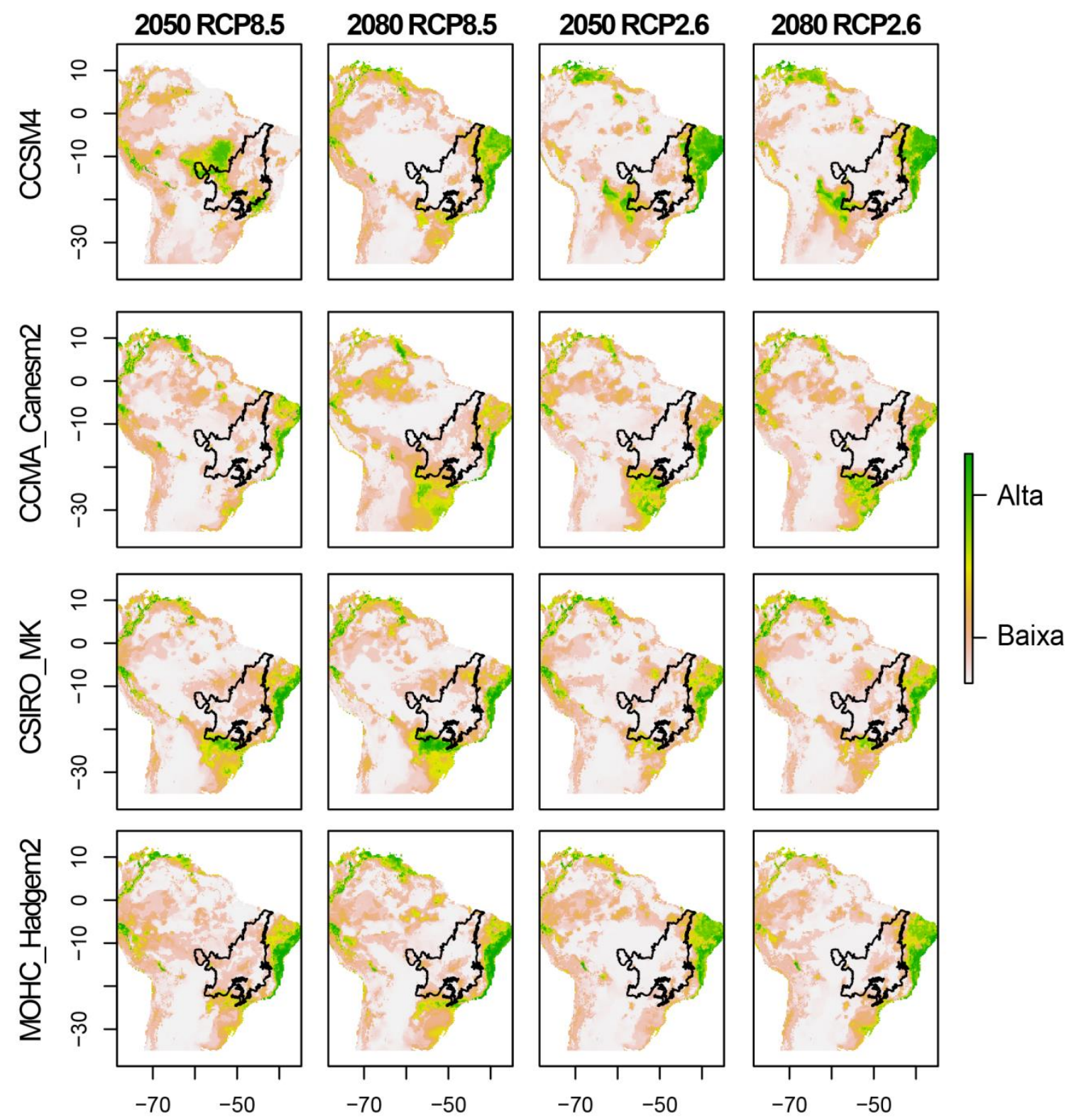
Callithrix penicillata
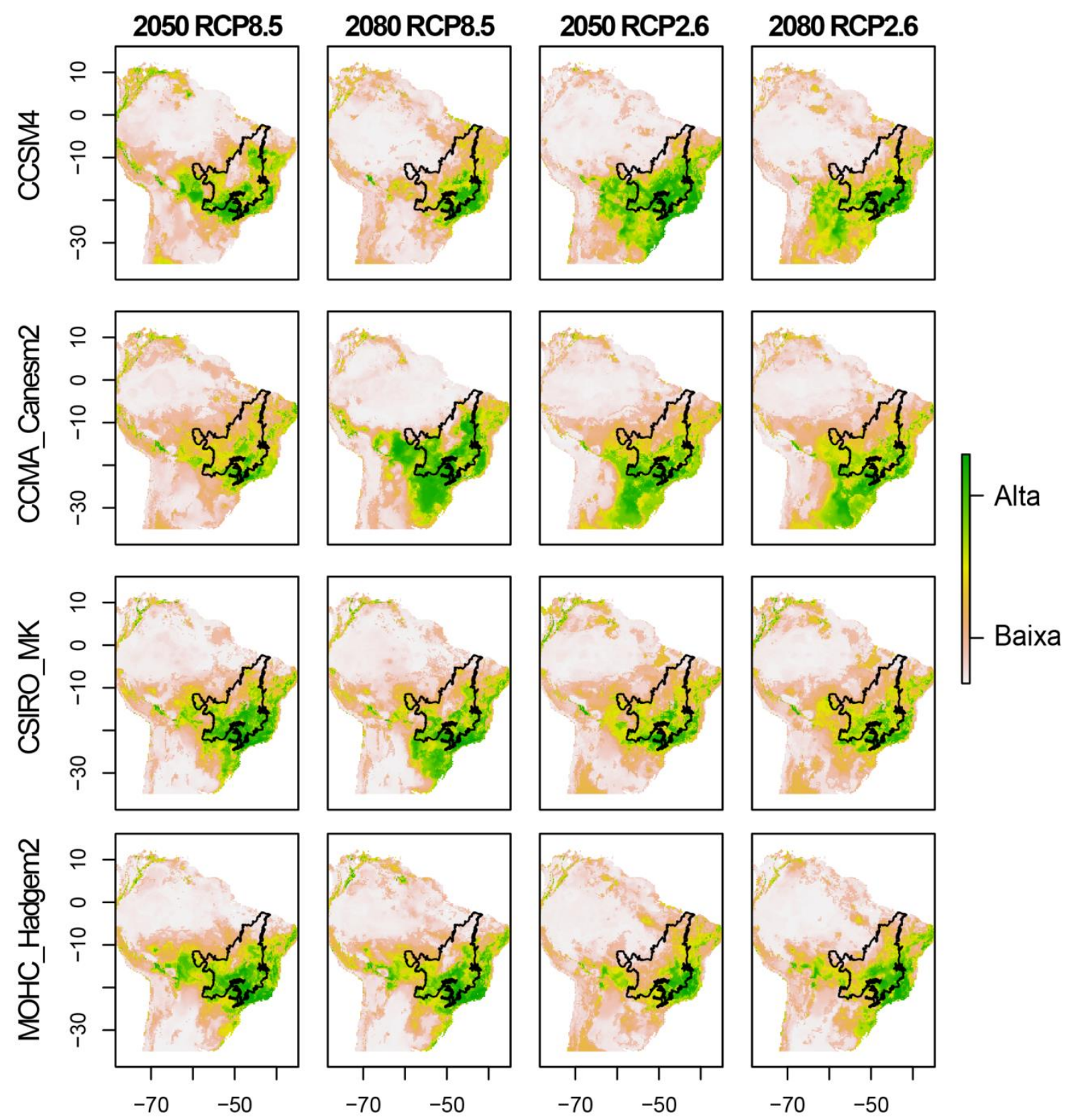
Mico melanurus
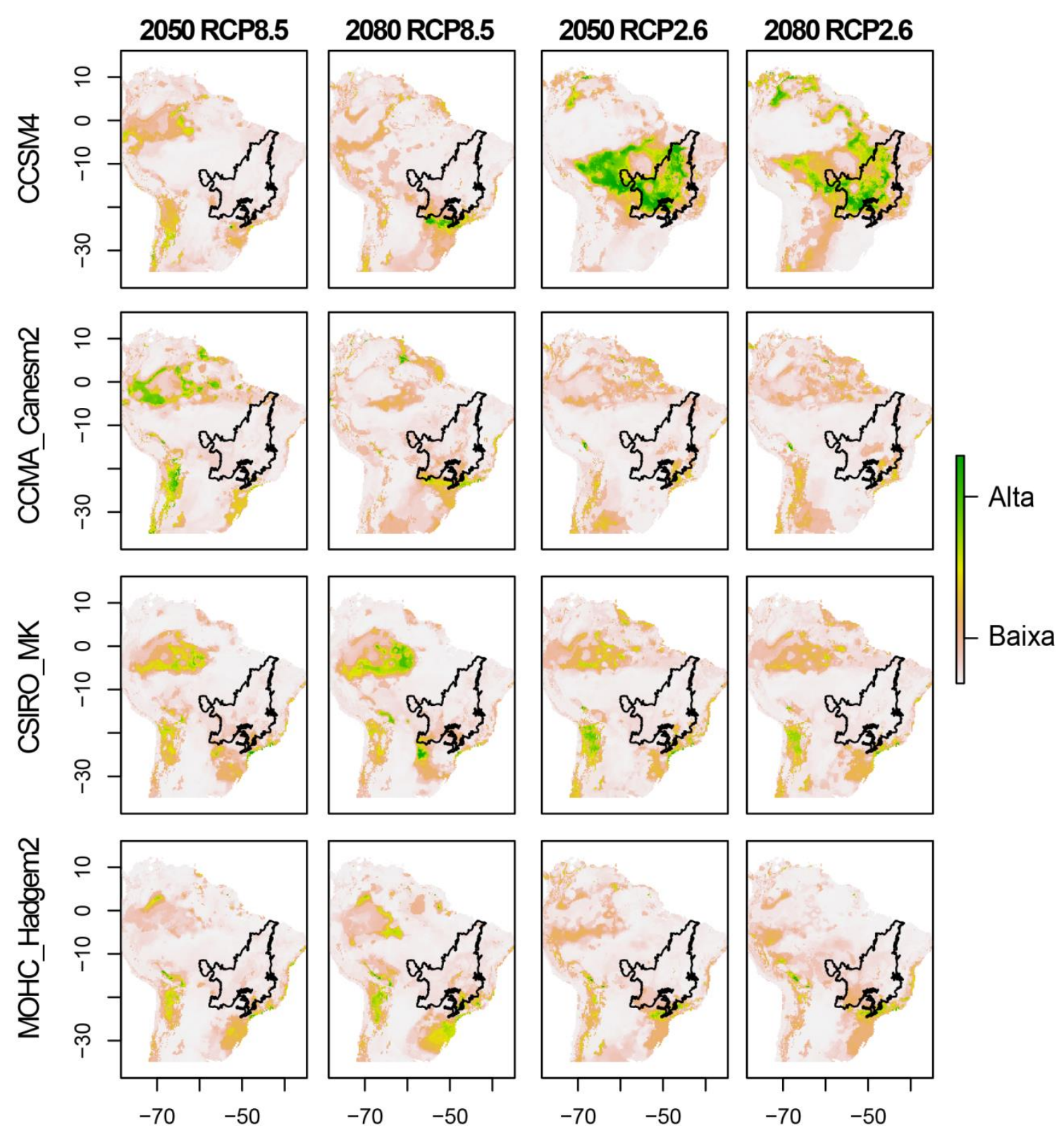
Sapajus cay
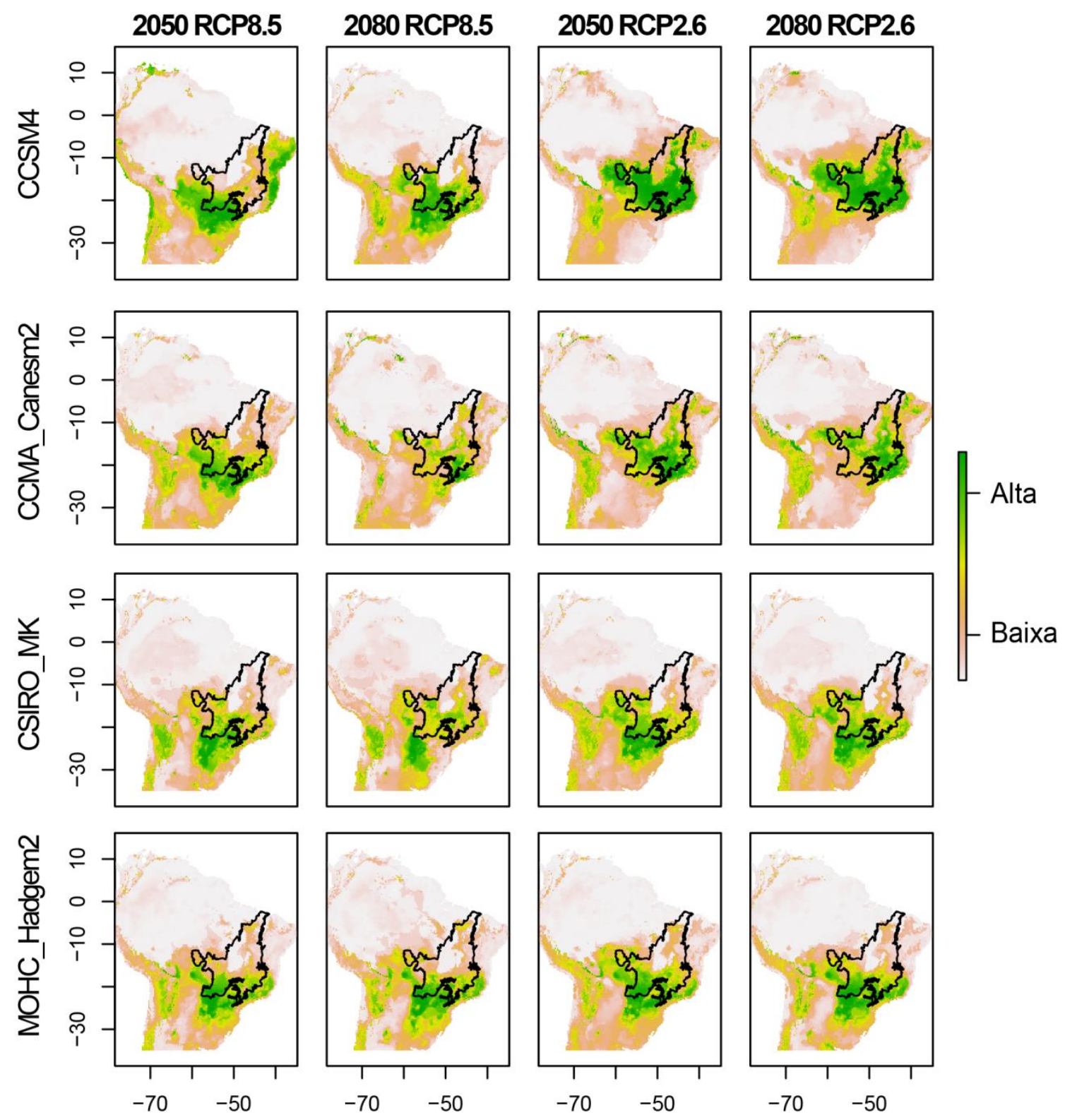
Sapajus libidinosus
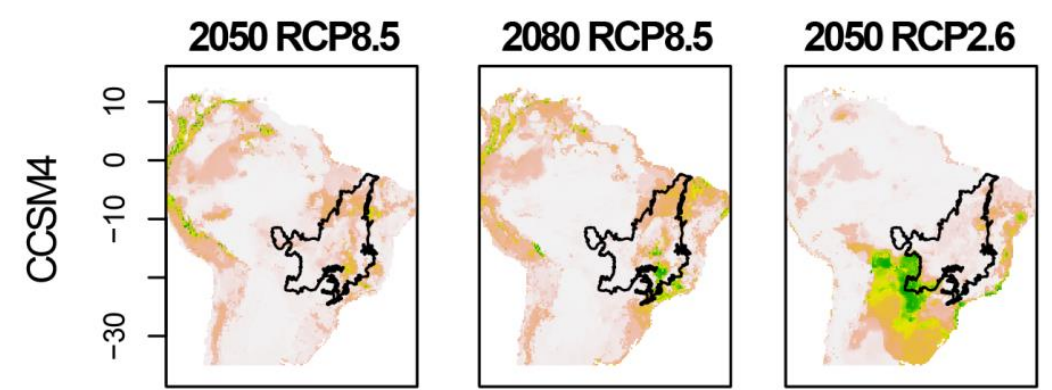

2080 RCP2.6
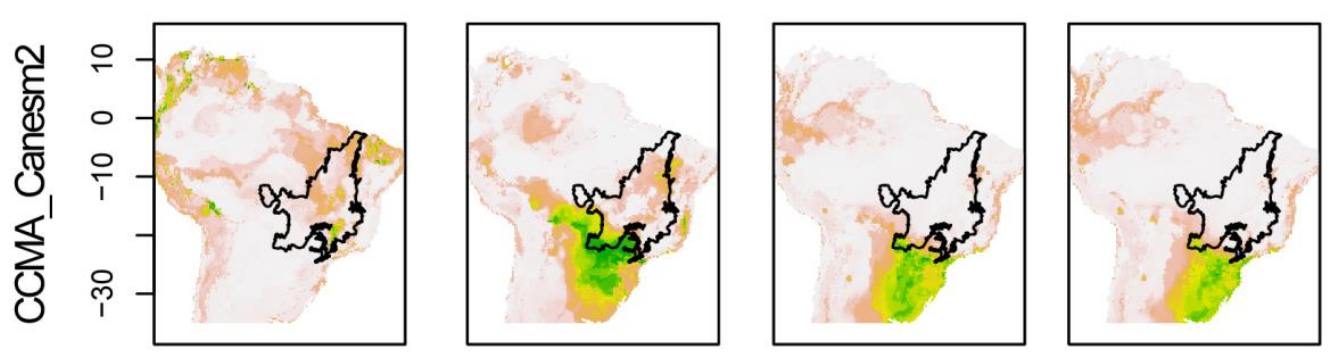

- Alta
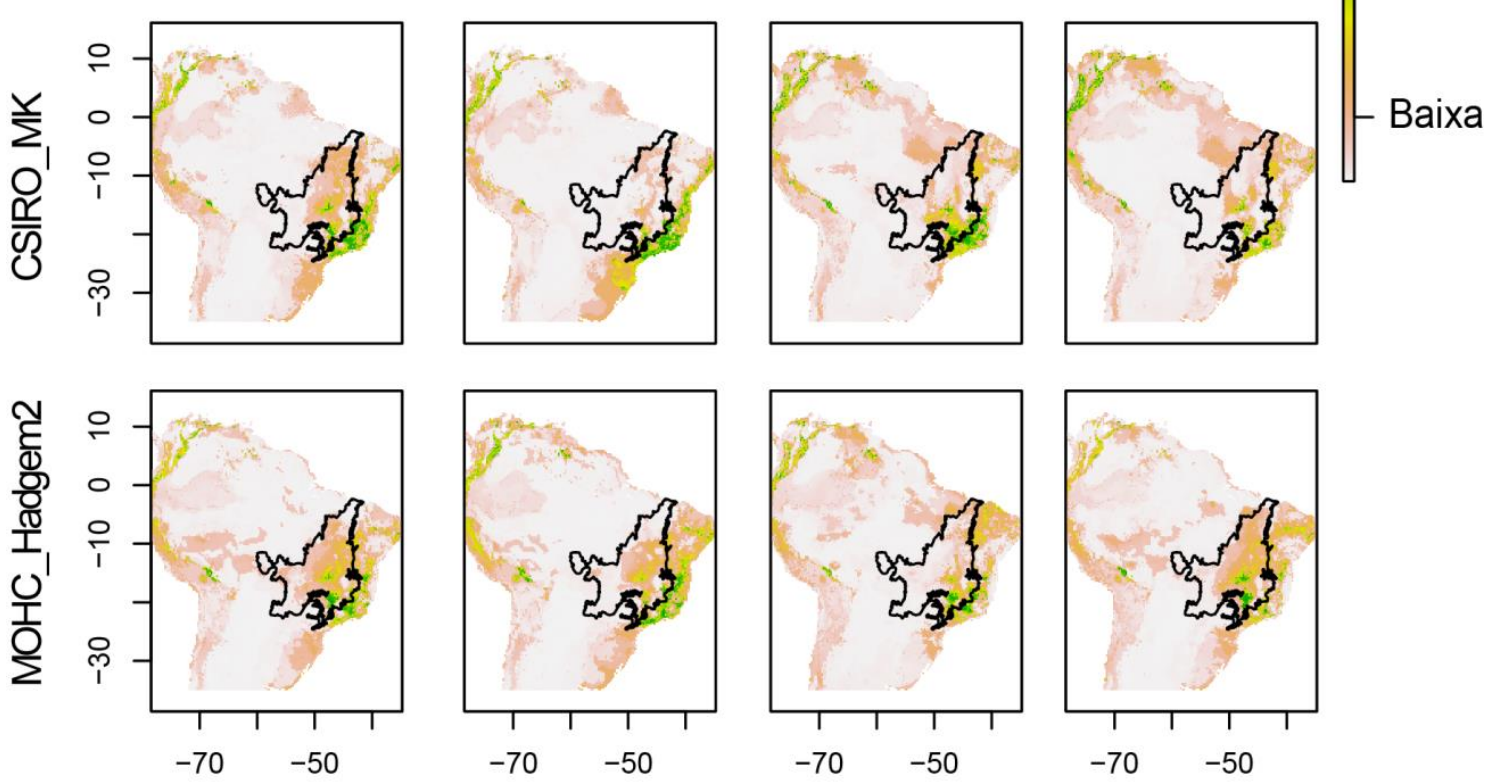


\section{Sapajus nigritus}
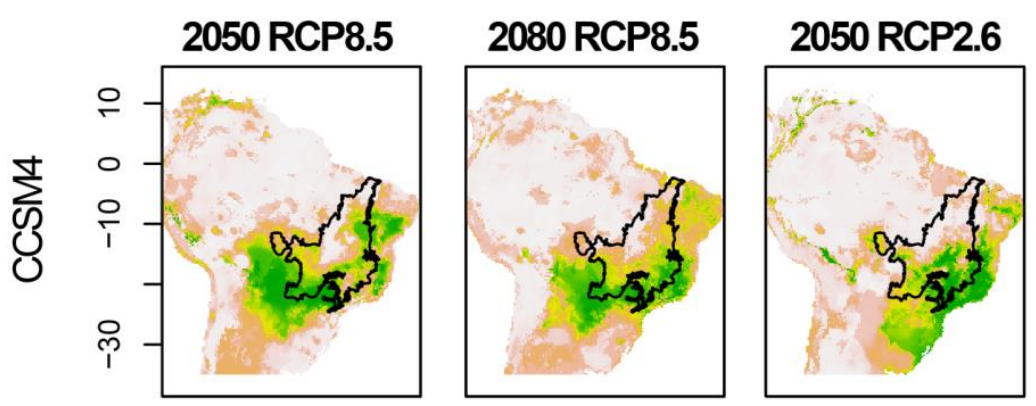

2080 RCP2.6
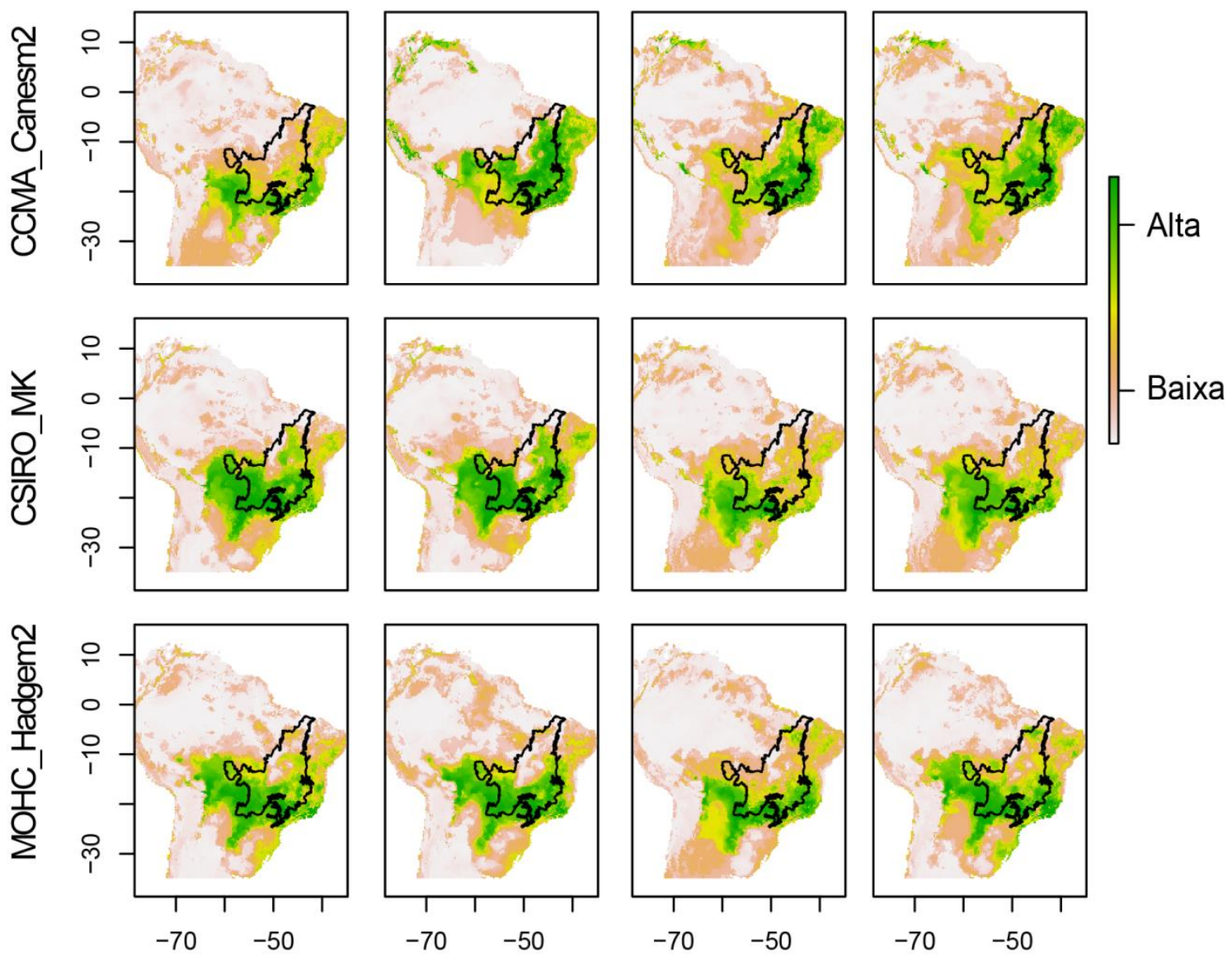
Sapajus xanthosternos
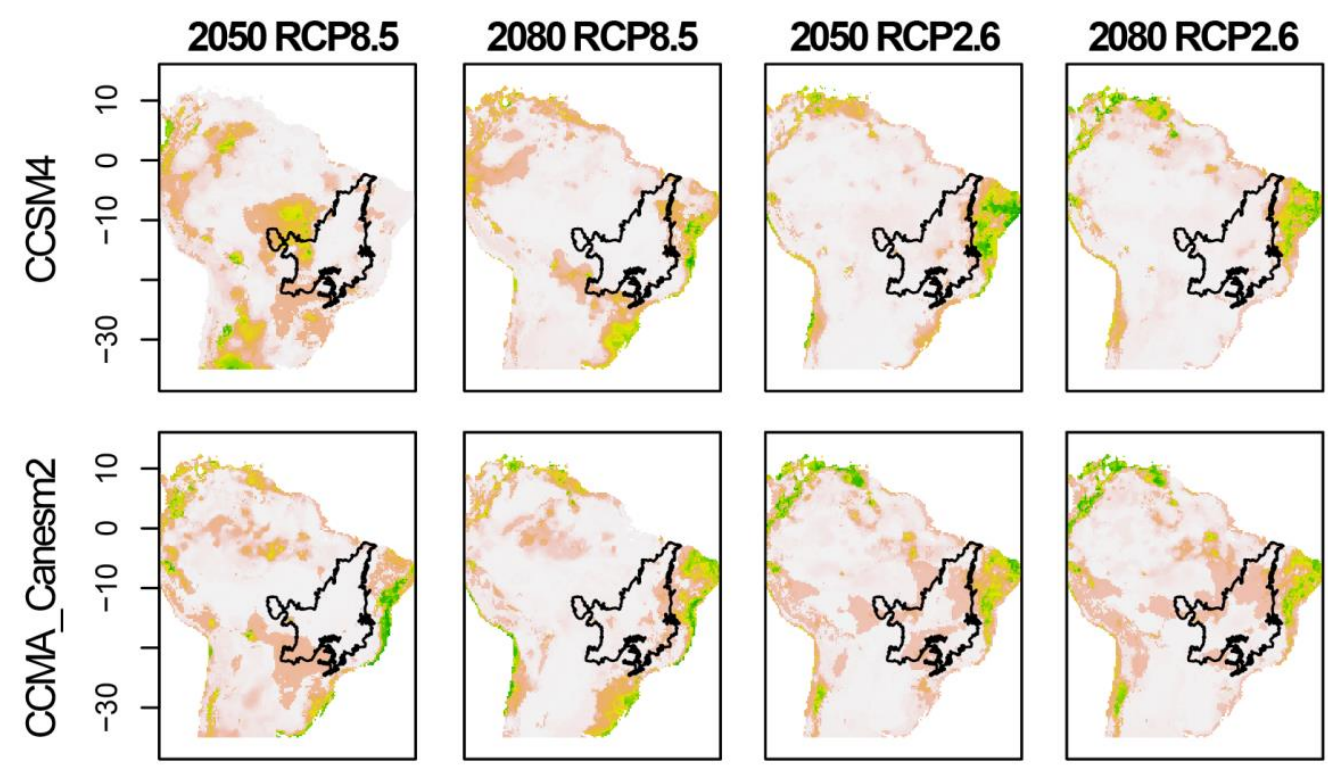

Alta
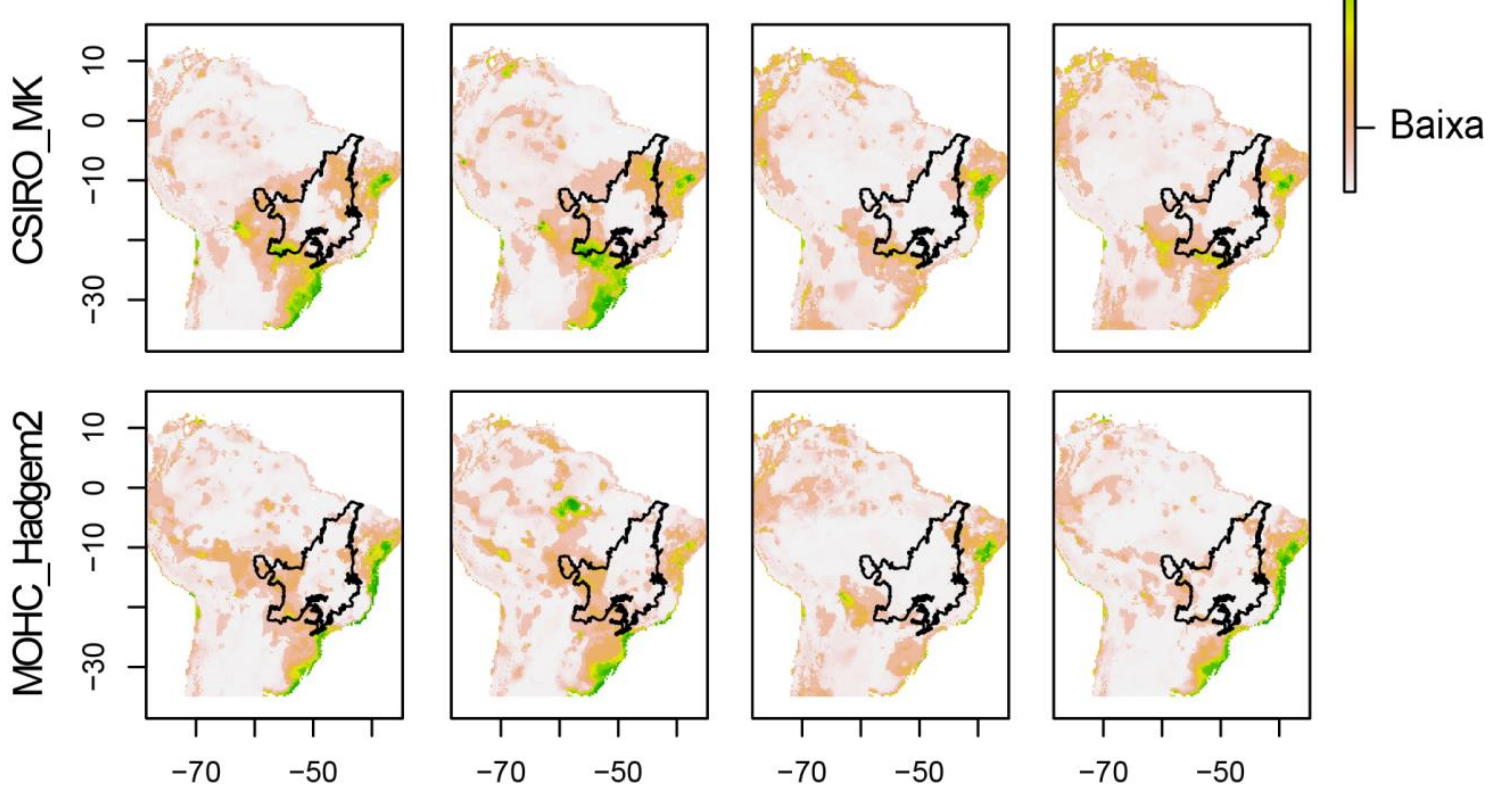

Figura A.3. Figuras representando as projeções de adequabilidade climática para as espécies de primatas analisadas nos diferentes modelos AOGCM (CCSM4, CCMA_Canesm2, CSIRO_MK e MOHC_Hadgem2) e cenários climáticos (RCP2.6 para 2050 e 2080 e RCP85 para 2050 e 2080). 


\section{Anexo 4. Lista das localidades de registro de primatas do Cerrado com suas coordenadas geográficas.}

Tabela A.1. Número de registros de ocorrência por espécie de primata

\begin{tabular}{lc}
\hline Espécie & Número de ocorrências \\
\hline Alouatta belzebul & 231 \\
Alouatta carya & 319 \\
Alouatta guariba & 365 \\
Aotus azarae azarae & 91 \\
Aotus azarae infulatus & 72 \\
Callicebus donacophillus & 38 \\
Callicebus nigrifrons & 68 \\
Callicebus personatus & 171 \\
Callithrix geoffroyi & 197 \\
Callithrix jacchus & 199 \\
Callithrix penicillata & 165 \\
Mico melanurus & 85 \\
Sapajus cay & 92 \\
Sapajus libidinosus & 53 \\
Sapajus nigritus & 358 \\
Sapajus xanthosternos & 97 \\
\hline
\end{tabular}

Cada linha apresenta longitude e latitude em graus decimais e em seguida as localidades

\section{Alouatta belzebul}

$-48,17 ;-3,76$; Rancho Cauaxi, Paragominas, PA

-48,25; -5,02; Complexo Celmar , Reserva de Celmar S. A. (Indústria de Celulose e

Papel), Vila Nova dos Martírios e São Pedro da Água Branca, MA

-49,64; -3,85; Lago de Tucuruí, Pará, PA

$-48,55 ;-0,69$; Fazenda Santa Maria, PA

-35,16; -7,05; Fazenda Pacatuba, Sapé, PB

-35,04; -7,16; Açude dos Reis, Santa Rita, PB

-34,99; -7, Mata de Jacuípe, Santa Rita, PB

-35,1; -7,19; Açude Cafundó, Cruz do Espírito Santo, PB

-34,99; -7,09; Usina Santana, Santa Rita, PB

$-35,93 ;-9,32$; Murici, AL

-36,13; -9,92; Usina Sinimbú, AL

$-35,22 ;-7,1$; Faz, Pacatuba, CE

$-45,25 ;-5,5$; Barra do Corda, MA

$-49,12 ;-5,35$; Marabá, PA

$-47,48 ;-5,53$; Imperatriz, MA

$-48,67 ;-6,42 ;$ Xamboiá, GO

$-50,25 ;-6,17$; Serra Norte, Carajás, PA

$-50,5 ;-5,83$; Serra Carajás, Cobre, PA 
-51,87; -6,63; Igarapé Maguari, PA

$-47,45 ;-0,77$; Maracanã, PA

$-47,4 ;-1,2$; Nova Timboteua, PA

-47,1; -1,55; Alto Igarapé Pedral, Ourém, PA

$-47,32 ;-3$,Paragominas, PA

$-48,98 ;-2,3$; Piratuba, PA

$-45,69 ;-3,99$; Santa Luzia, MA

-44,33; -4,74; São Benedito, Bom Jardim, CE

$-43,45 ;-2,62$; Miritiba, MA

-49,64; -4,09; Canoal, PA

-49,58; -4,27; Vila Brado, PA

-47,49; -1,63; São Miguel, PA

$-49,63 ;-4,55$; Chiqueirinho, PA

-49,47; -4,55; Jacundá, PA

-49,13; -4,5; Jacundá, PA

-49,73; -3,83; Vale do Caraipé, PA

-49,65; -4,03; Igarapé Araparí, PA

-49,61; -3,84; Timbozal, PA

$-49,49 ;-4,87$; Saúde, PA

$-49,53 ;-4,42$; Ilha Tocantins, PA

-48,85; -1,37; Ponta de Pedras, Marajó, PA

-49,92; -0,17; Igarapé Tapereba, Chaves, PA

$-50,82$; -1,95; Pracupy-Portel, Portel, PA

$-55,87 ;-1,75$; Oriximiná, PA

$-49,65 ;-3,84$; Rio Tucurú, PA

$-47,78 ;-1,67$; Rio Carpim, PA

-49,58; -0,03; Ilha Mexicana, PA

-48, 13; -0,8; Rio Muriá, Vigia, PA

-48,2; -1,97; Rio Acará, PA

-48,58; -0,28; Livramento, Marajó, PA

-50,02; -0,85; Igarapé Cururú, Marajó, PA

-36,02; -10,Usina Siminbu, AL

-35,2; -7,06; Fazenda Pacatuba, Sapé, PB

-35,94; -9,31; Serra Branca, Murici, PB

$-35,53 ;-8,71$; Usina Sacramento, Água Preta, PE

-35,01; -6,37; Mata da Estrela, Baía Formosa, RN

$-51,71$; -1,99; Estação Científica Ferreira Penna (ECFPn) no interior da FLONA de

Caxiuanã, Melgaço, PA

-35,17; -6,73; Reserva Biológica Guaribas, Mamanguape, PB

$-35,17$; -6,73; Reserva Biológica Guaribas, Mamanguape, PB

$-49,63 ;-3,84$; Lago de Tucuruí, PA

$-49,62 ;-3,88$; Rio Tocantins, Tucuruí, PA

$-35,21 ;-7,07$; Sapé, PB

$-47,32 ;-3,02$; Paragominas, PA

$-35,21 ;-7,06 ;$ Sapé, PB

$-46,4 ;-3,76$; Reserva Indígena Caru, MA

$-49,64 ;-3,85$; Rio Tocantins, PA

$-35,17 ;-6,72$; Mamanguape, PB

$-35,53 ;-8,71$; Mata do Engenho Sacramento, Água Preta, PE

-49,65; -3,85; Reservatório da UHE- Tucuruí, PA 
$-35,18 ;-6,73$; Reserva Biológica Guaribas, Mamanguape, PB

$-35,18 ;-6,73$; Mamanguape, PB

-35,18; -6,73; Rebio Guaribas, Mamanguape, PB

-51,72; -2,07; Estação Científica Ferreira Penna, PA

$-35,83$; -9,25; Serra Branca, Murici, AL

-35, 16; -7,05; Fazenda Pacatuba- Usina Santa Helena, PB

-35,52; -8,71; Água Preta, PE

$-45,27 ;-5,6$; Aldeia S. Pedro, MA

$-48,53 ;-1,49$; BelÚm, PA

$-45,32 ;-4,01$; Boa Vista, MA

$-50,26 ;-1,81$; Boiuçu, PA

$-44,35 ;-4,74$; Bom Jardim, PA

-55,1; -2,82; Caxiricatuba, PA

-49,08; -4,59; Estr.Belém-Marabá, PA

-47,41; -3,34; Estr.Belém-Brasília, PA

-48,93; -1,39; Faz. Cedro, R. javari, I. Marajó; Ponta de Pedra, PA

$-48,88 ;-1,36$; Faz. S. Joaquim, R. Javari, I. Marajó; Ponta de Pedra, PA

$-51,1 ;-6,76$; Gradaús, PA

-47,15; -1,52; Ig. Pedral Alto, Ourém, PA

-47,13; -1,54; Ig. Pedral Alto Santa fé em Deus, Ourém, PA

$-50,05 ;-0,13$; Ig. Taperebá, Chaves, PA

$-47,5 ;-5,53$; Imperatriz, MA

-59,85; -3,17; Lgo.Janaucá, AM

-49,17; -5,35; Marabá, PA

$-47,47 ;-0,77$; Maracanã, PA

-55,68; -4,08; Monte Cristo, PA

$-47,4 ;-1,23$; Nova Timboteua, mata, Ig. Burrinho; PA

$-47,41 ;-3$,Paragominas, PA

$-53,48 ;-1,8$; Prainha, PA

-45,87; -1,88; Rio Maracacumé, MA

$-50,62 ;-3,07 ;$ R. Pracupy, Portel, PA

$-50,-6$,Serra Carajás, Ser.N(N2); PA

$-50,43$; -5,58; Serra Carajás, Ser.N; PA

-45,66; -3,91; Santa Luzia, MA

$-48,19 ;-1,14$; Santo Antônio, PA

-49,63; -3,83; área do Lago da Barragem/r. tocantins, Tucuruí, PA

$-49,54 ;-4,12$; Canoal, Tucuruí, PA

-49,65; -4,33; Chiqueirinho, Tucuruí, PA

$-49,71 ;-3,8$; I. da Bandeira, Tucuruí, PA

-49,67; -3,79; I. Tocantins, Tucuruí, PA

-49,67; -3,78; Ig. Arapari, Tucuruí, PA

-49,63; -4,1; Ig. Canoal, Tucuruí, PA

-49,67; -3,81; Ig. Maguari, Tucuruí, PA

-49,12; -4,44; Jacundá, Tucuruí, PA

-49,12; -4,48; Jacundá, Tucuruí, PA

-49,66; -3,78; Tucuruí, PA

$-49,68 ;-3,78$; Tucuruí, PA

-49,63; -4,15; Morixaba, Tucuruí, PA

-49,64; -3,79; Rampa, Tucuruí, PA

-49,65; -4,33; S. Miguel, Tucuruí, PA 
-49,59; -4,29; S. Miguel, Tucuruí, PA

-49,21; -5,33; Saúde, Tucuruí, PA

-49,49; -4,53; Saúde, Tucuruí, PA

-49,51; -4,51; Saúde, Tucuruí, PA

-49,65; -3,84; Timbozal, Tucuruí, PA

-49,67; -4,03; Timbozal, Tucuruí, PA

$-49,65 ;-3,9$; Vale do Caraipê, Tucuruí, PA

-49,57; -4,14; Vila Brabo, Tucuruí, PA

-36,58; -10,29; Usina Sinimbú, Manimbu, AL

$-48,21 ;-7,19$; Xamboiá, posto indígena. Carajás; Araguaina, GO

-52,77; -4,62; Igarapé Bom Jardim, Paissandu; PA

-47,34; -3, Igarapé Itinga, Paragominas, PA

-49,12; -5,37; Marabá, PA

$-52,77 ;-4,67$; Par. Do Bom Jardim, Paissandu; PA

-49,65; -3,86; Reservatório de Tucuruí - Ilha Germoplasma, PA

$-49,64 ;-3,85$; Ilha Germoplasma, PA

-49,71; -3,91; Reserva Hidroelétrica de Tucuruí, Tucuruí, PA

$-49,71 ;-3,96$; Germoplasma, PA

$-35,21 ;-7,06$; Sapé, PB

$-46,75 ;-1,88$; Fazenda Amanda, Viseu, PA

$-49,66 ;-3,89$; Ilha de Germoplasma (Usina Hidrelétrica de Tucuruí), PA

$-46,76 ;-3,74 ;$ MA

$-52,-6,69$; Ilha Grande, PA

-53,36; -4,24; Mundo Novo, banco direito rio Iriri; PA

-51,38; -1,73; Estação Científica Ferreira Penna, PA

-50,33; -3,83; Fazenda Arataú, PA

$-49,93 ;-4,47$; Terra Indígena Parakanã, PA

$-51,64 ;-2,07$; Caxiuanã, AM

$-47,58 ;-2,92$; Fazenda Vitoria, AM

$-48,93 ;-2,89$; Tailândia, AM

-47,83; -1,64; São Domingos do Capim, AM

$-47,46 ;-1,74$; Irituia, AM

$-46,75 ;-3,21 ;$ Gurupí, AM

$-49,71 ;-4,31$; Novo Repartimento, AM

$-60,1 ;-3,73$;

-34,98; -6,43; Mata da Estrela, Baía Formosa, RN

-35,08; -6,65; Mata Cajaranas ou Aguas Claras, Rio Tinto, PB

-35,18; -6,73; Rebio Guaribas, Mamanguape, PB

$-35,1 ;-6,97$; Dois Rios, Santa Rita, PB

-35,08; -7,02; Jacuipe ou Sucupira, Mamanguape, PB

-34,95; -7,01; Santana ou Gargaú, Mamanguape, PB

$-35,02 ;-7,15$; Reis, Mamanguape, PB

-35,13; -7,04; Asentamento Santa Helena, Mamanguape, PB

-35,15; -7,04; Pacatuba, Sapé, PB

-35,09; -7,19; Cafundó, Cruz do Espírito Santo, PB

-34,96; -7,03; Mata paú do Pau Brasil, Santa Rita, PB

-35,4; -8,71; Engenho Sacramento, Palmares, PE

-35,79; -9,27; Esec Murici, Flexeiras, AL

-35,98; -9,52; RPPN Santa Tereza, Atalaia, AL

-36,06; -9,88; Mata do junco, São Miguel dos Campos, AL 
-36,18; -10,04; Usina Coruripe, Coruripe, AL

-36,23; -10,02; Usina Coruripe, Coruripe, AL

$-36,3 ;-10,01$; Usina Coruripe, Coruripe, AL

$-35,23 ;-7,1$; Mata de Capatuba, Sapé, PB

-50,64; -5,92; Rio Itacaíunas, PA

$-55,6$; -4,92; Itaituba, Br. 165,Santarem-Cuiaba, Zona Sul; Para

-55,33; -4,42; Itaituba, Guariba, Km. 25,Transamazonica Hwy; Para

$-58,25$; -3,3; Rio Amazonas, Lago do Baptista; Amazonas

$-55,5 ;-3,67$; Rio Tapajos, Fordlandia; Para

$-55,23 ;-3,37$; Rio Tapajos, west bank, Arara; Para

$-55,22 ;-3,42$; Rio Tapajos, east bank, Tavio; Para

$-54,95 ;-3,35$;

$-54,67$; -4, Santarem, Br 165,Santarem To Cuiaba, Km. 212; Para

$-54,95 ;-3,35$;

-52,37; -3,67; Altamira, Ca. 54 Km SSW, Island In Rio Xingu Near E Bank; Para

-57,82; -2,77; Rio Amazonas, Ilha de Urucurituba; Para

$-55,08 ;-2,9$; Rio Tapajos, right bank, Tapaiuna; Para

-55; -3; Rio Tapajos; Prainha, Para

$-49,5 ;-1$, Ilha de Marajo, Para

$-54,93 ;-3,35 ;$ Pará

$-51,47$; -1,7; Estação científica Ferreira Pena, Pará

-50,33; -3,83; Fazenda Arataú, Pará

-49,93; -4,47; Território Indigena Parakanã, Pará

$-35,93 ;-9,32$; Murici, Alagoas

$-36,13 ;-9,92$; Usina Sinimbú, Alagoas

$-45,27 ;-5,53$; Aldeia S. Pedro, Barra do Corda, Maranhão

$-47,48 ;-5,53$; Imperatriz, Maranhão

-49,46; -4,79; Igarapé Araripe (126 km sul da barragem Tucuruí, rio Tocantins); Pará

$-51,9 ;-3,6 ;$ Rio Bacajá (boca), Pará

$-48,48 ;-1,43$; Belém, Pará

-49,5; -2,25; Cametá, Pará

-49,78; -4,08; 30 km sul da barragem Tucuruí, m.d. rio Tocantins; Canoal, Pará

$-47,78 ;-1,67$; Rio Capim, Pará

$-50,25 ;-6,17$; Serra dos Carajás, Pará

$-50,-0,08$; Ilha Caviana, Pará

$-49,73 ;-3,83$; Vale do Caraípe, Pará

$-49,47 ;-4,55 ; 70$ km sul da barragem Tucuruí, m.d. rio Tocantins; Chiqueirinho, Pará

$-50,18 ;-0,65$; Igarapé Cururú, Ilha de Marajo, Pará

-52,38; -3,65; Cachoeira do Espelho (Rio Xingu), Pará

$-47,67 ;-2,12$; BR 010,km 93; Pará

$-49,38 ;-5,68 ;$ m.e. Rio Tocantins, Itupiranga, Pará

$-49,48 ;-4,65 ; 120$ km sul da barragem Tucuruí, m.d. rio Tocantins; Jacundá, Pará

-48,58; -0,28; Livramento, Ilha de Marajo, Pará

-49,58; -4,27; Igarapé Maguari (68 km sul da barragem Tucuruí), Pará

-49,17; -5,35; Marabá, Pará

$-47,45 ;-0,77$; Maracanã, Pará

$-47,24 ;-1,2 ;$ Nova Timboteua, Pará

-47,1; -1,55; Rio Guamá, Ourém, Pará

$-48,98 ;-2,3$; Piratuba, Pará

-47,32; -3,Paragominas, Pará 
-49,12; -0,83; Ponta de Pedras, Rio Ariri; Ilha de Marajó, Pará

-50,82; -1,95; Rio Pracupy, Portel, Pará

$-49,67 ;-2,82 ;$ m.d. rio Tocantins, Santo Antônio, Pará

$-49,21 ;-5,3 ; 170$ km sul da barragem Tucuruí, m.e. Rio Tocantins, Saúde; Pará

-52,67; -3,9; Largo do Souza, Rio Xingu; Pará

-49,12; -0,17; Igarapé Tapereba, Chaves; Ilha de Marajó, Pará

$-49,53 ;-4,42$; Ilha Tocantins, Pará

-49,67; -4,42; Usina Hidrelétrica de Tucuruí, Pará

-35,22; -7,1; Fazenda Pacatuba, Sapé, Paraíba

$-35,52 ;-8,7$; Usina Sacramento, Água Preta, Pernambuco

-35,02; -6,39; Mata da Estrela, Baía Formosa, Rio Grande do Norte

$-48,22 ;-7,35$; Araguaina, Tocantins

-48,58; -3,66; Fazenda Rio Capim, Paragominas, Pará

$-48,78 ;-2,6$; Maçaranduba, PA

\section{Alouatta caraya}

$-53,83 ;-11,42$; Alto Xingu, MT

$-52,25 ;-15,9$; Aragarças, GO

$-53,71 ;-23,23$; Bar. do Ivinheima, I. Santana; MS

$-50,54 ;-16,47$; Bar do R.S. Domingos, GO

-43,47; -14,33; Carinhanha, BA

-49,27; -8,25; Conceição do Araguaia, R. Araguaia; PA

-57,66; -19,02; Corumbá, MS

-44,2; -11,83; Cotegipe, Riacho dos Neves; Ingazeiro, BA

-54,35; -20,45; Faz Brilhante, Campo Grande, MT

-46,35; -18,57; Faz. Cágado, Presidente Olegário, MG

-54,95; -28,42; Faz. Casamata (Eng. Velho), S. Luíz Gonzaga, RS

$-44,42 ;-18,75$; Faz. Chorão, Curvelo, MG

-55,82; -20,45; Faz. Cruzeiro, Aquidauana, MS

-48,96; -16,96; Faz. Dois Irmãos, Bela Vista, GO

-43,72; -14,33; Faz. P. de José, Bambuzada, BA

$-53,84 ;-23,37$; I. Bandeirantes, PR

$-55,67 ;-10,27$; I. de Taiamã, MT

$-53,68 ;-23,3$; I. de Longa, Querência do Norte, PR

$-54,02 ;-23,72$; I. Sete Quedas, PR

$-51,51 ;-20,64$; Itapurá, SP

$-56,39 ;-20,23$; Miranda, MS

$-57,78 ;-16,73$; Paratubal, MT

-59,06; -15,29; R. das Mortes, S. Domingos, MT

$-48,65 ;-20,47$; R. Grande (Mato Grosso de Barretos), SP

$-53,95 ;-23,68 ;$ R. Paracai, PR

$-46,39 ;-12,88$; R. Palma, GO

$-48,52 ;-14,47 ;$ S. José do Tocantins, GO

-57,74; -15,93; S. Luíz de Cáceres, MT

$-57,65 ;-16,09$; Salobra, MT

$-44,91 ;-17,32$; Ser. De Sta. Maria, Pirapora, MG

-44,45; -10,91; Sta. Rita de Cássia, Maracaju, BA

-53,77; -23,32; Alto Rio Paraná, PR 
-54,98; -29,56; Cerro dos Negros, São Francisco de Assis, RS

-55,-29,59; Cerro dos Negros, São Francisco de Assis, RS

-47,83; -21,16; Projeto Barba Negra, Ribeirão Preto, SP

-43,52; -19,26; Morro do Pilar, MG

-53,41; -22,73; Rio Paraná, PR

-56,29; -29,62; Estância Casa Branca, Alegrete, RS

-53,67; -29,04; São Xavier, Tupanciretã, RS

-53,27; -22,77; Alto Rio Paraná, Porto Rico, PR

-53,76; -23,38; Rio Paraná, Mata do Bugio; Icaraíma, PR

-53,32; -22,77; Rio Paraná, Porto Rico, PR

-56,27; -29,61; Estabelecimento Nossa Senhora da Conceição, Alegrete, RS

-53,74; -23,35; Paredão das Araras, Porto Figueira, PR

$-53,47 ;-22,82$; Florestas do Alto Rio Paraná,

-47,83; -15,71; Jardim Zoológico de Brasília, Brasília, DF

-47,91; -15,67; Jardim Zoológico de Brasília, Brasília, DF

$-56,65 ;-18,98$; Mancha de floresta na Fazenda Nhumirim, MS

$-53,84 ;$-29,08; Tupanciretã, RS

$-53,97 ;-29,12$; Lajeado do Celso, Tupanciretã, RS

$-53,89$; -29,05; Coxilha Bonita, Tupanciretã, RS

-54,04; -29,12; Santa Luzia, Tupanciretã, RS

-54,25; -28,96; Espinilho Grande, Tupanciretã, RS

$-53,65 ;-29$, São Xavier, Tupanciretã, RS

$-53,77$; -28,95; Batú, Tupanciretã, RS

$-54,11 ;-28,93$; Santo Inácio, Tupanciretã, RS

-53,84; -29,07; Perímetro Urbano, Tupanciretã, RS

-53,85; -29,07; Perímetro Urbano, Tupanciretã, RS

-54,06; -28,93; São Bernardo, Tupanciretã, RS

$-53,77$; -28,95; Batú, Tupanciretã, RS

-53,88; -28,86; Estância Grande, Tupanciretã, RS

-54,14; -29,12; Santa Luzia, Tupanciretã, RS

-54,31; -29,06; Passo da Lage, Tupanciretã, RS

-53,91; -29,2; Aguapé, Tupanciretã, RS

$-53,95 ;-29$, Bocaverá, Tupanciretã, RS

-53,23; -22,76; Porto Rico, PR

-53,32; -22,71; Porto Rico, PR

$-53,32 ;-22,77$; Ilha Mutum, Porto Rico, PR

$-55,77 ;-29,81$; Alegrete, RS

$-55,8 ;-29,84$; Alegrete, RS

$-47,27 ;-16,19$; Aproveitamento Hidrelétrico de Queimado, MG

-53,37; -27,93; Fazenda do Angico, Fortaleza dos Valos, RS

$-53,51 ;-26,57$; Guaraciaba, SC

$-47,81 ;-21,2$; Ribeirão Preto, SP

-47,85; -21,14; Ribeirão Preto, SP

-47,88; -21,15; Ribeirão Preto, SP

-47,81; -21,23; Ribeirão Preto, SP

$-47,94 ;-21,2$; Ribeirão Preto, SP

-47,-22,43; Zoológico de Mogi-Mirim, SP

$-48,15 ;-21,19$; Fragmento de mata ciliar, Barrinha, SP

$-47,8 ;-21,17$; Ilha de mata urbana no Parque Municipal Morro de São Bento, Ribeirão

Preto, SP 
$-53,69 ;-26,51$; Ouro Verde, Guaraciaba, SC

$-48,17 ;-21,17$; Barrinha, SP

$-48,06 ;-21,24$; Barrinha, SP

$-47,81 ;-20,99 ;$ Jardinápolis, SP

-47,94; -15,9; Mata do Monjolo (Reserva Ecológica do IBGE), DF

-53,19; -28,98; Chácara São Pedro, Campos Borges, RS

-53,61; -29,17; Fazenda do Angico, Fortaleza dos Valos, RS

-53,48; -29,11; Fazenda Santa Maria, Boa Vista do Incra, RS

$-53,57$; -28,93; Fazenda Itapevi, Boa Vista do Incra, RS

-53,42; -28,86; Fazenda da Lagoa, Boa Vista do Incra, RS

-54,09; -29,36; Lageado do Celso, Tupanciretã, RS

-53,72; -29,1; Granja Costa Beber, Bozano: (Ijuí), RS

-53,6; -28,82; Fazenda Três Capões, Cruz Alta, RS

$-54,36$; -29,43; Propriedade particular, Jari, RS

-53,68; -29,23; Fazenda Coxilha Bonita, Julio de Castilhos, RS

$-54,92$; -28,72; Gr. Escoteiros Guaranis, Bossoroca, RS

$-54,92 ;-29,18$; Terreno particular urbano, Santiago, RS

-55,31; -29,19; Zona urbana Praça Central, São Francisco de Assis, RS

$-53,22 ;-28,8$; Propriedade particular, Fortaleza dos Valos, RS

$-55,49 ;-30,24 ;$ São Carlos, RS

$-55,66 ;-30,86$; RS

$-55,57 ;-30,84 ; \mathrm{RS}$

$-55,79 ;-30,1 ; \mathrm{RS}$

$-55,75 ;-29,9$; Alegrete, RS

-55,8; -29,95; Alegrete, RS

$-53,2 ;-28,77$; Fortaleza dos Valos, RS

$-53,47 ;-28,77$; Boa Vista do Incra, RS

$-54,9 ;-29,16 ;$ Santiago, RS

$-54,92 ;-20,73$; Terenos, MS

$-57,65 ;-19$, Morro do Rachid, Corumbá, MS

$-54,87 ;-20,39$; Terenos, MS

-47,8; -21,17; Parque Municipal Morro de São Bento, Ribeirão Preto, SP

-57,02; -19,58; Base de estudos do Pantanal da Universidade Federal de Mato Grosso

do Sul, Corumbá, MS

-57,68; -19,03; Baia Negra, Corumbá, MS

-48,36; -14,08; Usina Hidrelétirca de Serra da Mesa, Goiânia, GO

-56,28; -29,62; Estância Casa Branca, Alegrete, RS

-49,2; -16,64; Parque Zoológico de Goiânia -PZG, Goiânia, GO

-47,61; -19,99; Mata dos Dourados, Fazenda Mandioca; Conquista, MG

$-47,92 ;-15,58 ;$ DF

$-48,08 ;-15,75 ; \mathrm{DF}$

$-47,36 ;-20,49$; Franca, SP

$-61,13 ;-12,4 ;$ Pequeno/K-48,RO

-53,42; -22,78; Ilha Mutum, Porto Rico, PR

-44,63; -10,23; Angico, Parnaguá, PI

$-62,78 ;-12,54 ;$ AC

$-55,8 ;-20,48$; Aquidauana, Aquidauana, MS

$-55,8 ;-20,47$; Aquidauana, Aquidauana, MS

-55,79; -20,46; Hotel Portal Pantaneiro, Aquidauana, MS

-56,2; -19,48; Fazenda Rio Negro, Aquidauana, MS 
-55,81; -20,47; Areeiro Saara, Anastácio, MS

-54,63; -20,44; Área Militar, Campo Grande, MS

-54,61; -20,41; Parque Estadual Matas do Segredo (PEMS), Campo Grande, MS

-47,84; -20,52; PCH Anhanguera, São Joaquim da Barra, SP

$-56,1 ;-21,45$; Fazenda São Marcos, Guia Lopes da Laguna, MS

$-56,16 ;-21,48 ;$ Jardim, MS

$-56,63 ;-21,1$; Parque Nacional da Serra da Bodoquena, Bodoquena, MS

$-56,67 ;-20,53$; Morraria do Sul, Bodoquena, MS

-57,65; -19,05; Maçico de Urucum, Corumbá, MS

-57,48; -18,06; Serra do Amolar, Corumbá, MS

-55,81; -20,48; Rio Aquidauana, Anastácio, MS

$-63,32 ;-12,32$; REBIO Guaporé, RO

-53,59; -28,81; Passo dos Alemães, Cruz Alta, RS

-53,67; -28,74; Bejamim Not, Cruz Alta, RS

-53,69; -28,78; Urupú, Cruz Alta, RS

$-42,32 ;-8,43$; PARNA Serra da Capivara, PI

-54,87; -20,73; Fazendas Nova Querência Nova Esperança, MS

-47,8; -21,58; Estação Ecológia de Jataí, Luís Antônio, SP

-46,64; -23,55; São Paulo, SP

$-47,81 ;-21,18$; Ribeirão Preto, SP

$-48,97 ;-22,32$; Bauru, SP

$-46,07 ;$; 23; Igaratá, SP

-47,47; -21,18; Altinópolis, SP

$-49,02 ;-22,6$; Agudos, SP

$-51,1 ;-20,98$; Mirandópolis, SP

$-48,9 ;-22,47 ;$ Agudos, SP

$-48,92 ;-22,5 ;$ Agudos, SP

$-51,1 ;-20,82$; Pereira Barreto, SP

$-50,97 ;-20,87$; Pereira Barreto, SP

$-47,45 ;-21,18$; Cajuru, SP

$-48,57 ;-20,58$; Barretos, SP

$-50,83 ;-20,98$; Valparaíso, SP

$-51,51 ;-20,65$; Itapura, SP

-59,95; -15,01; Vale do Guaporé, Vila Bela da Santíssima Trindade, MT

-56,62; -16,26; Fazenda Santa Inês, Pocone, MT

-53,27; -22,77; Ilha Mutum, Porto Rico, PR

$-49,62 ;-25,49$; Bugre, Balsa Nova, PR

$-60,03 ;-37,16$; Buenos Aires

-58,42; -34,58; Plaza Italia, Ciudad Autónoma de Buenos Aires

-57,95; -34,92; La Plata, Buenos Aires

$-59,05 ;-28,5$; R Parana, Bella Vista, near; Corrientes

$-59,22 ;-28,04$; Florencia, General Obligado, Santa Fe

$-59,63 ;-28,62$; Isla Laurelty, General Obligado, Santa Fe

-59,47; -28,74; Arroyo Los Amores, cerca de Arroyo Seibal; General Obligado, Santa

$\mathrm{Fe}$

-58,24; -28,47; Estancia Rincón de Luna, Concepción; Concepción, Corrientes

$-58,09 ;-28,03$; Laguna del Potrero 1., Corrientes

$-57,2 ;-28,55$; Reserva Natural del Ibera, Laguna de Pellegrini, en el bosquecito al sur

del destacamento de los guardaparques.; CORRIENTES

-59,16; -27,58; Arroyo Guaycurú, ruta 11; San Fernando, Chaco 
-58,68; -27,05; Las Palmas, Bermejo, Chaco

$-58,1 ;-27,92$; Manantiales, Mburucuyá, Corrientes

$-58,81 ;-27,48$; Corrientes

$-58,64 ;-27,31$; Isla Brasilera, Bermejo, Chaco

$-58,78 ;-27,49$; Corrientes, Capital, Corrientes

$-57,66 ;-27,76 ; 15$ km al SO de Gral. Paz, camino a Iberá; San Miguel, Corrientes

-55,99; -27,8; Río Aguapey, Ituzaingó, Corrientes

$-54,42$; -27,32; Reserva Natural Educativa Colonia Benitez, Chaco; Chaco

$-60,82 ;-26,67$; Sin localidad precisa, Chaco

$-60,89 ;-26,03$; Chaco

-59,37; -26,3; El Colorado, Pirané, Formosa

$-59,61 ;-26,81$; Puente colgante, Chaco

$-59,-27$, Rio de Oro, Chaco

$-58,81 ;-26,81$; Las Palmas y El Perdido, Bermejo, Chaco

-58,63; -26,24; San Francisco de Laishi, Laishi, Formosa

-54,8; -26,62; Arroyo Doradito, Parque Provincial Isla Caraguatay; Montecarlo,

Misiones

-54,17; -26,37; Cuartel Río Victoria, km 273,Ruta 14,Reserva de Uso Múltiple Cuartel

Río Victoria; Guaraní, Misiones

-58,18; -25,12; Monte Destacamento Estero Poí, Formosa

$-58,13 ;-25,17$; Dto de Gpques. Laguna Blanca, Formosa

$-58,89 ;-25,86$; Gran Guardia, Pirané, Formosa

$-58,18 ;-25,12$; Piquete Portada, Formosa

$-57,99 ;-25,09 ;$ Zanja soro, Formosa

$-54,16$; -25,68; Ruta 101. Seccional Yacuy., Misiones

-54,16; -25,57; Andresito. Límite Este. Lote 29,Misiones

-54,46; -25,65; Sendero Yacaratiácute; desvío de la botera. Cuadro redes, luego

cascadita $500 \mathrm{~m}$; Misiones

$-54,45 ;-25,69$; Final del paseo superior, Misiones

$-54,21$; -25,68; Palmital a $1 \mathrm{~km}$ de ruta 101,al Norte, altura curva Palo Rosa.; Misiones

$-54,44$; -25,7; Area Cataratas. 20 metros antes del lugar de venta de agua de Garganta;

Misiones

$-54,45 ;-25,69$; Islote frente a la toma de agua., Misiones

-54,26; -25,94; Río Urugua-í (Curso medio), 30 km Puerto Bemberg; Iguazú, Misiones

$-54,16$; -25,66; Límite Este. Frente a las chacras numero 1 y 21,Misiones

$-54,45 ;-25,69$; Camping ATE, Misiones

$-54,55 ;-25,63$; Mbocaí detras de EA, Misiones

$-54,48 ;-25,69$; Palmital del cruce, Misiones

-54,46; -25,66; Final del Sendero Macuco, Misiones

$-54,43 ;-25,7$; Camping Nandu, Misiones

$-54,22$; -25,67; A 2 km de la Ruta 101,al Norte, en la primera Laguna (entre palmital).;

Misiones

-54,33; -25,58; Iguazu Superior, entre Apepu y boca del Santo Domingo, 10 min (aprox.

$800 \mathrm{~m})$ antes de llegar a la boca del Santo Domingo.; Misiones

$-54,43 ;-25,72 ; \mathrm{RN} 101$. Entre $\mathrm{A}^{\mathrm{o}}$ Nandu y $\mathrm{A}^{\mathrm{o}}$ Central, Misiones

$-54,25 ;-25,67$; Ruta Nacional $N^{\circ} 101$, Misiones

$-54,54 ;-25,65$; Sendero Yacaratia. Repetidora canal 12., Misiones

-54,34; -25,6; Río Iguazú; Superior, Misiones

-54,15; -25,66; Límite Este. Lote21. Chacra Mulauka, Misiones

-54,6; -25,9; Arroyo Urugua-I, 10 miles up Arroyo from Rio Parana; Misiones Province 
-54,32; -25,57; Entre seccional Apepu y Arroyo Santo Domingo, Misiones

$-54,17 ;-25,68$; Yacuy, Misiones

-54,14; -25,68; RN101 y Acceso a la Escuela No 436. Cabure-1́. Andresito, Misiones

-54,23; -25,68; PNI. RN N\&deg; 101. Palo Rosa caído (en picada palmiteros), Misiones

$-54,54 ;$ - 25,76; RN No 12. PECOM. Borde de bañado (mano izquierda, a Posadas),

Misiones

$-54,51 ;-25,65 ; \mathrm{RN} \mathrm{N}^{\mathrm{o}}$ 12,Misiones

-54,47; -25,69; Parcelas Proyecto de Restauracion (320m SUR, 370m OESTE de entrada $(0,0)$ Bloque II.; Misiones

-54,15; -25,65; Límite Este. Lote 22. Chacra Mulauka, Misiones

$-57,2 ;-23,7$; Puerto Ybapobo, San Pedro

$-42,98 ;-22,43$; Teresopolis, Rio de Janeiro

$-57,7 ;-16,75$; Descalvado (=Descalvados Ranch), Mato Grosso

$-56,18 ;-16,14$; Cuiaba Brazil,

$-64,95 ;-14,78 ; 70 \mathrm{~km} \mathrm{~N}$ from: Trinidad,

-64,82; -13,07; Aguadulce, Mamore, El Beni

$-68,68 ;-12,97$; Mouth of Quebrada Juliaca, on Rio Heath, ca. 50 river km S Puerto

Pardo; Madre de Dios Department

-40,22; -11,52; Santa Rita; Maracuja, Bahia

$-43,72 ;-14,33$; Bambuzada, Bahia

$-43,78 ;-14,3$; Carinhanha, Bahia

$-44,2 ;-11,83$; Cotegipe, Bahia

-44,87; -11,Ibipetuba (antiga Santa Rita de Cássia), Bahia

$-43,75 ;-14,33$; Malhada, Bahia

$-52,21 ;-15,95$; m.d. Rio Araguaia, Aragarças, Goiás

$-46,93 ;-13,85$; Cana Brava, Goiás

$-49,27 ;-16,68$; Goiânia, Goiás

$-46,93 ;-12,45$; Inhumas, Goiás

$-48,45 ;-14,45$; Niquelândia, Goiás

-47,87; -12,55; Rio Palma, Goiás

-47,77; -12,72; Barra do Rio São Domingos, Goiás

-48,-12,42; São Miguel, Goiás

-45,97; -9,1; Alto do Rio Parnaíba, Maranhão

-57,73; -11,42; Rio Arrais (Alto Rio Xingu), Mato Grosso

-57,68; -16,07; São Luíz do Cáceres, Mato Grosso

-52,42; -12,03; Jacaré, Alto Rio Xingu; Mato Grosso

$-57,65 ;-19$,Maracaju, Mato Grosso

$-57,73 ;-16,4$; Paratuval, Mato Grosso

$-58,47$; -15,85; Porto Esperidião, Mato Grosso

$-51,4 ;-13,5$; São Domingos (rio das Mortes), Mato Grosso

$-57,42 ;-16,85$; Ilha Taimã, Mato Grosso

-54,6; -20,43; Campo Grande, Mato Grosso do Sul

$-57,65 ;-19,02$; Corumbá, Mato Grosso do Sul

-53,67; -22,97; Barra do Ivinheima (Ilha Santana), Mato Grosso do Sul

-56,37; -20,25; Campo Miranda, Mato Grosso do Sul

$-52,17 ;-21,25$; Fazenda Santa Bárbara (Santa Inês), Mato Grosso do Sul

-56,52; -20,18; Salobra, Mato Grosso do Sul

$-48,18 ;-18,63$; m.d. Rio Grande, Araguari, Minas Gerais

$-44,43 ;-16,2$; Brasília de Minas, Minas Gerais

$-44,42 ;-18,75$; Curvelo, Minas Gerais 
-44,95; -17,35; Pirapora, Minas Gerais

-46,42; -18,42; Presidente Olegário, Minas Gerais

-49,27; -8,3; Rio Araguaia, Conceição do Araguaia, Pará

-54,58; -25,55; Foz do Iguaçu, Paraná

$-54,25 ;-24,07$; Guaíra, Paraná

$-53,5 ;-23,07$; Querência do norte, Paraná

$-53,93 ;-23,47$; Rio Pacaraí, Paraná

$-42,75 ;-8,42$; Parque Nacional Serra da Capivara, Piauí

-45,78; -9,18; Chapada das Mangabeiras, Piauí

-55,13; -29,55; São Francisco de Assis, Rio Grande do Sul

-54,97; -28,4; São Luiz Gonzaga, Rio Grande do Sul

-48,97; -21,13; Catanduva, São Paulo

-51,52; -20,67; Itapura, São Paulo

-53,26; -22,76; Porto Rico, Paraná

$-53,17 ;-22,71$; Porto Rico, Paraná

-53,34; -22,74; Mato Grosso do Sul

$-53,08 ;-22,65$; Porto Rico, Paraná

$-53,14 ;-22,65$; Mato Grosso do Sul

$-53,9 ;-23,87$; Parque Nacional Ilha Grande, Altônia, Paraná

-54,26; -24,08; PARNA Ilha Grande, Guaíra, Paraná

$-53,61 ;-23,4$; Icaraíma, Paraná

$-53,04 ;-22,74$; Marilena, Paraná

-52,99; -22,77; Nova Londrina, Paraná

-54,59; -25,55; PARNA do Iguaçu, Paraná

$-53,73 ;-23,51$; PARNA Ilha Grande, Paraná

-53,27; -22,77; Porto Rico, Paraná

-53,18; -22,72; Porto São José, Paraná

-53,48; -23,08; Ilha Longa, Querência do Norte, Paraná

-53,88; -23,76; PARNA Ilha Grande, São Jorge do Patrocínio, Paraná

-53,22; -22,82; São Pedro do Paraná, Paraná

$-42,49 ;-8,78$; Parque Nacional da Serra da Capivara, Piaui

$-54,79 ;-28,7$; Gr. Escoteiros Guaranis, Bossoroca, RS

-54,87; -29,17; Terreno particular urbano, Santiago, RS

-55,16; -29,09; Zona urbana Praça Central, São Francisco de Assis, RS

$-58,17 ;-25,98$; Guaycolec ranch, Formosa

$-58,68 ;-27,5$; Misiones

$-55,61 ;-19,51$; Fazenda Santa Emília, Mato Grosso do Sul

-63,06; -17,79; Jardim Botânico de Santa Cruz, Santa Cruz - Bolívia

-56,27; -29,61; Estabelecimento Nossa Senhora da Conceição, Alegrete, RS

$-58,68 ;-27,5$; basin of the Riachuelo river, Corrientes province

$-58,67 ;-27,33$; Isla Brasilera,

-59,28; -29,62; Estância Casa Branca, Alegrete, RS

$-59,5 ;-26,92$; Puerto Bermejo, Argentina

-53,76; -23,38; Mata do bugio, Icaraíma, PR

-53,27; -22,77; Porto Rico, PR

$-47,61$; -19,99; Mata dos Dourados, Fazenda Mandioca; Conquista, MG

$-61,45 ;-13,44 ;$ RO

-48,23; -20,02; Reserva Volta Grande, Conceição das Alagoas, MG

-43,52; -19,26; Parque Nacional Serra do Cipó, Morro do Pilar, MG

-43,68; -18,36; João Vasse, Gouveia, MG 


\section{Alouatta guariba}

-51,4; -25,14; Paredão das Araras, Porto Figueira, PR

$-49,02 ;-24,47$; Apiai, SP

-52,62; -27,1; Faz. Boa Esperança, S. Domingos; Chapecó, SC

-49,49; -27,8; Faz. Campos Novos, Bom Retiro, SC

-52,6; -27,07; Faz. Faxinal, S. Domingos; Chapecó, SC

-50,43; -28,67; Faz. Monte Alegre, Bom Jesus, RS

$-48,83 ;-25,99$; Faz. R. Turvo, Garuva, SC

-53,37; -23,16; Faz. Sta. Catarina, Querência do Norte, PR

-49,78; -21,63; Faz. Varjão, Lins, SP

$-49,52 ;-27,06$; Ibirama, SC

$-51,3 ;-30,29$; Barra do Ribeiro, RS

$-46,51 ;-23,37 ; \mathrm{SP}$

$-46,53 ;-23,38 ;$ SP

-51,22; -30,02; Morro da Extrema, Porto Alegre, RS

$-46,65 ;-23,54$; São Paulo, SP

-50,77; -30,1; Estiva-Viamão, RS

-51,22; -30,05; Morro da Extrema, Porto Alegre, RS

-49,23; -26,9; Centro de Pesquisas Biológicas de Indaial - CEPESBI, Indaial, SC

-47,06; -22,91; Mata Ribeirão Cachoeira, Distrito de Sousas e Joaquim Egídio;

Campinas, SP

$-52,98 ;-30$, Cachoeira do Sul, RS

-49,64; -25,58; Chácara Payquerê do Bugre, Balsa Nova, PR

$-42,14 ;-19,79$; Caratinga, MG

$-51,18 ;-29,92$; Porto Alegre, RS

-44,23; -22,52; Área de Relevante Interesse Ecológico Floresta da Cicuta, RJ

-44,33; -22,63; Área de Relevante Interesse Ecológico Floresta da Cicuta, RJ

-44,15; -22,4; Floresta da Cicuta, RJ

$-41,82 ;-19,73 ; \mathrm{MG}$

$-47,11 ;-22,81$; Reserva de Santa Genebra, SP

$-47,11 ;-22,8$; Reserva de Santa Genebra, SP

$-42,57 ;-19,72 ; \mathrm{MG}$

$-48,36 ;-24,36 ;$ SP

-48,63; -26,25; Vila da Glória; Centro de Estudos e Pesquisas Ambientais (CEPA), São

Francisco do Sul, SC

$-50,29 ;-26,21$; São João da Urtiga, SC

$-50,95 ;-30,38$; RS

$-51,14 ;-28,27 ; \mathrm{RS}$

$-50,09 ;-29,22 ; \mathrm{RS}$

$-51,55 ;-28,2$; Lagoa Vermelha, RS

$-51,72 ;-28,51$; São Jorge, RS

$-51,69 ;-28,54$; Guabijú, RS

$-51,81 ;-30,85$; Camaquã, RS

-53,7; -29,72; Campo de Instrução de Santa Maria, RS

-53,81; -29,68; Santa Maria, RS

$-51,13$; -29,91; Porto Alegre, RS

-51,04; -30,16; Viamão, RS 
$-51,05 ;-29,97$; Porto Alegre, RS

$-51,05 ;-29,99$; Porto Alegre, RS

-48,65; -26,24; Distrito do Saí, São Francisco do Sul, SC

-49,67; -25,51; Chácara Payquerê. Distrito do Bugre, Balsa nova, PR

-49,67; -25,49; Chácara Payquerê. Distrito do Bugre, Balsa nova, PR

-49,65; -25,51; Chácara Payquerê. Distrito do Bugre, Balsa nova, PR

$-49,65$; -25,5; Chácara Payquerê. Distrito do Bugre, Balsa nova, PR

$-49,65$; -25,49; Chácara Payquerê. Distrito do Bugre, Balsa nova, PR

$-50,97 ;-30,38 ;$ RS

-46,91; -23,2; Associação Mata Ciliar, Jundiaí, SP

$-46,32 ;-22,85$; Extrema, RS

$-47,97 ;-23,1 ;$ Santa Genebra, SP

-48,05; -24,02; Ribeirão Cachoeira, SP

$-43,05 ;-22,83$; Boa Vista, RJ

$-44,25 ;-20,61$; Bela Fama, MG

$-48,42 ;-24,35$; Ribeirão Grande, SP

$-51,2 ;-30,05$; Morro da Extrema, Porto Alegre, RS

$-51,2 ;-23,2$; Mata Doralice, Ibiporã, PR

$-51,23 ;-30,12$; Morro do Osso, Porto Alegre, RS

$-51,16 ;-28,22 ; \mathrm{RS}$

-53,73; -29,76; Campo de Instrução de Santa Maria (CISM), Santa Maria, RS

-53,73; -29,71; Campo de Instrução de Santa Maria (CISM), Santa Maria, RS

-49,06; -26,94; Parque São Francisco de Assis, Blumenau, SC

$-51,05 ;-23,27$; Mata Doralice, Ibiporã, PR

$-50,47 ;-21,4$; Biriguii, SP

-51,12; -29,82; Parque Zoológico de Sapucaia do Sul, RS

$-51,05 ;-30,25$; Águas Claras, Viamão, RS

$-52,07$; -31,Rio Camaquã, Cristal, RS

-53,46; -24,96; Rio Camaquã, Cascavel, RS

$-52,32 ;-31,17$; Rio Camaquã, Canta Galo, RS

$-47,97 ;-24,11 ; \mathrm{SP}$

$-51,02 ;-23,27$; Mata Doralice, Ibiporã, PR

-44,55; -22,42; Trilha Maromba-Lamego, RJ

$-49,24 ;-26,9$; Morro Geisler, Indaial, SC

$-51,23 ;-30,03$; Porto Alegre, RS

$-41,77 ;-20,42 ; \mathrm{MG}$

$-43,87 ;-21,67 ; \mathrm{MG}$

-49,3; -26,92; Centro de Pesquisas Biológicas de Indaial, Indaial, SC

-49,45; -26,99; Centro de Pesquisas Biológicas de Indaial, Indaial, SC

-45,49; -22,76; Fazenda São Sebastião do Ribeirão Grande - Serra da Mantiqueira,

Pidamonhangaba, SP

$-48,16 ;-22,57$; Fazenda Barreiro Rico, SP

$-43,06 ;-22,49 ; \mathrm{RJ}$

-42,98; -22,54; Fazenda Guapimirim, RJ

-49,65; -25,48; Chácara Payquerê:Centro de Educação Ambiental e apoio à Pesquisa,

localizada no Distrito do Bugre; Balsa Nova, PR

-49,68; -25,49; Chácara Payquerê:Centro de Educação Ambiental e apoio à Pesquisa,

localizada no Distrito do Bugre; Balsa Nova, PR

$-48,85 ;-26,3$; Serra Dona Francisca, Juinville, SC

$-47,94 ;-25,11$; Cananéia, SP 
-46,96; -22,88; Reserva Santa Genebra, Campinas, SP

-43,29; -22,02; Mata Boa Vista, Fazenda Amazonas; Comendador Levy Gaparian, RJ

-42,17; -19,74; Fazenda Montes Carlos, Caratinga, MG

-47,72; -23,81; Fazenda João XXIII, Pilar do Sul, SP

$-43,35 ;-21,76$; Juiz de Fora, MG

$-51,1 ;-30,17$; Morro São Pedro, RS

$-42,14 ;-19,77 ; \mathrm{MG}$

$-42,46 ;-20,7 ; \mathrm{MG}$

$-42,25 ;-19,64$; entorno do Parque Estadual, MG

-40,7; -20,04; fragmento de mata em propriedades privadas, Santa Maria de Jetibá, ES

-42,56; -21,47; Macaé de Cima, RJ

$-41,81 ;$ - 21,86; Boqueirão da Mata na Serra Grande, RJ

$-48,13$; -22,67; Viraeiro/Tabatinguera (Fazenda Barreiro Rico), SP

$-44,63 ;-23,24$; Pedra Branca, Paraty, RJ

-42,22; -16,83; Fazenda Paiol, Virgem da Lapa, MG

-42,34; -16,8; Fazenda Santa Clara, Vila Piauí; Araçuaí, MG

-40,5; -19,77; Santa Tereza, ES

-40,48; -20,3; Reserva Florestal de Duas Bocas (RFDB), Cariacica, ES

-40,32; -20,35; Reserva Florestal de Duas Bocas (RFDB), Cariacica, ES

-40,5; -20,39; Reserva Florestal de Pedra Azul (REPA), Domingos Martins, ES

-40,13; -19,48; Reserva Florestal da Companhia Vale do Rio Doce (RCVRD), Linhares, ES

-40,1; -19,46; Reserva Florestal da Companhia Vale do Rio Doce (RCVRD), Linhares, ES

-39,81; -18,26; Conceição da Barra, ES

-41,06; -20,77; Reserva Florestal da Fazenda Montes Verdes (RFFMV), Vargem Alta, ES

-40,58; -19,92; Estação Biólogica de São Lourenço (EBSL), ES

-51,24; -30,01; Mata da Extrema, Porto Alegre, RS

-51,22; -30,06; Mata de Restinga do Lami, Porto Alegre, RS

$-53,65 ;-27,45$; Terra Indígena da Guarita, RS

$-53,92 ;-27,22$; Parque Estadual do Turvo, Derrubadas, RS

-50,58; -29,45; Floresta Nacional de São Francisco de Paula, São Francisco de Paula,

$\mathrm{RS}$

-46,74; -23,72; APA Municipal Capivari-Monos,

$-46,64 ;-23,76$; São Paulo, SP

$-45,56 ;-23,03$; Taubaté, SP

$-46,63 ;-23,87$; APA Capivari, SP

$-46,62 ;-23,51$; PE Alberto Lüfgren,

$-52,3 ;-22,54$; PE do morro do Diabo, SC

-54,98; -29,57; Cerro dos Negros, São Francisco de Assis, RS

$-54,34 ;-29,1$; Passo da Lage, RS

$-40,63 ;-19,54$; Colatinas, ES

-42,1; -19,23; Cór. Do Santo, Tarumarim, MG

-41,64; -19,63; Faz. Boca da Matá, Pocrane, MG

-39,75; -18,6; Faz. Da Besta, Mata da Besta; Conceição da Barra, ES

-40,08; -19,39; Faz. Dulcelândia, Mata Dulcelândia; Linhares, ES

-41,01; -20,42; Faz. S. José, Mata S. José; S. Domingos, ES

$-40,72 ;-17,08 ;$ Machacalis, MG

$-42,55 ;-19,65$; P.F. do R. Doce, MG 
-42,55; -19,89; R. Matipoó, MG

-41,26; -19,33; Resplendor, ES

-41,92; -19,92; Simonésia, Alegria, MG

-41,51; -17,86; Teófilo Otoni, MG

$-42,42 ;-20,67 ; \mathrm{MG}$

$-42,46 ;-20,69 ; \mathrm{MG}$

$-41,83 ;-19,83$; Fazenda Monte Claros, Caratinga, MG

-42,48; -20,75; Fazenda Neblina, MG

-42,4; -20,6; Fazenda Brigadeiro, MG

$-42,42 ;-20,64$; Ararica, MG

-42,45; -20,71; São Bento, MG

-41,98; -16,67; Estação Biológica de Caratinga, MG

-42,15; -19,75; Estação Biológica de Caratinga, MG

-43,79; -21,84; Lima Duarte, MG

-41,77; -16,61; Fazenda Santa Maria, Itinga, MG

-40,92; -16,89; Fazenda Esplanada, Pampa, MG

-40,65; -16,75; Fazenda Nova Esperança, Felizburgo, MG

-40,76; -16,64; Fazenda Córrego Seco, Joaima, MG

-40,81; -16,62; Fazenda Agua Fria, Felizburgo, MG

-40,69; -16,18; Fazenda Estancia Betania, Almenara, MG

-39,95; -16,Fazenda Serra Branca, Salto de Divisa, MG

$-40,17 ;-19$, Linhares, ES

$-40,14 ;-18,34$; Pinheiros, ES

-40,62; -15,25; Itambé, BA

-40,74; -15,46; Ribeirão do Largo, BA

$-42,53 ;-19,75 ; \mathrm{MG}$

-42,88; -20,75; Viçosa, MG

-38,88; -15,86; Proximidades de Belmonte, BA

-39,53; -15,95; Proximidades de Barreiras, Itapebí - estrada Belmonte; BA

-41,78; -19,62; Estação Biológica de Caratinga (sítio 6), MG

$-41,-16,43$; Jequitinhonha, MG

$-38,95 ;-15,68$; Fazenda Santa Clara, Canavieiras, BA

-47,-24,31; Juréia - Itatins, Peruíbe, SP

$-47,24 ;-23,96$; Juquitiba, SP

-47,96; -25,11; Ilha do Cardoso, Cananéia, SP

-40,61; -19,91; Floresta da Colônia São José, Santa Teresa, ES

-40,14; -18,34; Córrego do Veado, Pinheiros, ES

-40,58; -19,95; Santa Lúcia, Santa Teresa, ES

-39,7; -18,42; Itaúnas, Conceição da Barra, ES

-39,75; -18,58; Faz. da Besta (= Mata da Besta), Conceição da Barra, ES

-40,55; -19,85; Augusto Ruschi (ex- Nova Lombardia), Santa Teresa, ES

-40,5; -20,3; Duas Bocas, Cariacica, ES

-41,01; -20,41; PE Pedra Azul, Domingos Martins, ES

-40,65; -20,13; Porto Cachoeiro, R. Santa Maria; Santa Leopoldina, ES

-40,07; -19,42; Faz. Taquaral, m.dir. do R. Doce; Linhares, ES

-40,07; -19,33; Lagoa Juparanã, Linhares, ES

-40,6; -19,93; São Lourenço, Santa Teresa, ES

-40,63; -20,12; R. da Pedra, Santa Leopoldina, ES

-41,28; -20,15; Brejetuba, Brejetuba, ES

-39,95; -19,12; Linhares - CVRD, Linhares, ES 
-41,18; -20,59; PE Mata das Flores, Castelo, ES

-39,84; -18,73; São Mateus, ES

-40,64; -19,14; Faz. São José (= Mata de São José), São Domingos, ES

-41,-20,43; Faz. Montes Verdes (= Hotel Faz. Caezar Park, 24 km a SE da Sede); Venda

Nova do Imigrante, ES

-40,62; -20,1; Chaves, a $40 \mathrm{~km}$ do litoral; Santa Leopoldina, ES

-40,62; -19,12; Faz. Braço do Sul (= Afluente Branco do Sul), São Domingos, ES

-39,83; -18,25; Córrego Grande (ex- Faz.), Conceição da Barra, ES

$-40,62 ;-19,54 ;$ R. Doce, Colatina, ES

-41,02; -20,4; PE Pedra Azul, Domingos Martins, ES

-39,83; -19,62; R. Doce, m.esq., Regência; Linhares ?, ES

$-40,88 ;-19,5 ;$ Foz do R. Mutum (= Murutu ?), Baixo Guandu ?, ES

-41,47; -20,77; Engenheiro Reeve (= Rive), Alegre, ES

-40,58; -19,92; Córrego Santa Maria, Santa Teresa, ES

-39,75; -19,1; Faz. Dulcelândia (= Mata Dulcelândia), Linhares, ES

-40,08; -18,98; Sooretama, Linhares, ES

-40,12; -19,8; Aracruz Celulose, Aracruz, ES

-40,37; -19,83; Pau Gigante, Ibiraçu (antiga Pau Gigante), ES

-40,74; -20,06; Fragmento Florestal, Santa Maria de Jetibá, ES

-41,29; -20,76; FN de Pacotuba, Cachoeiro de Itapemirim, ES

-40,55; -19,17; Faz. Catete (= Mata Catete), São Domingos, ES

-40,63; -19,13; Faz. Dez de Agosto, São Domingos, ES

-40,09; -19,46; Faz. Goitacazes - Mata Experimental, Rio Doce; Linhares, ES

-45,08; -23,28; Estrada Santa Virgínia/Cunha, Trilhas Itamambuca e Rio dos Veados;

São Luís do Paraitinga, SP

-47,78; -24,05; São Miguel Arcanjo, SP

$-48,83 ;-24,52 ;$ Apiaí, SP

$-46,65 ;-23,42$; Serra da Cantareira, São Paulo, SP

$-49,74 ;-21,68 ;$ Lins, SP

$-45,09 ;-22,53$; M' Boi Mirim, São Paulo, SP

-46,06; -23,8; Distrito de Paranapiacaba, Santo André, SP

$-46,63 ;-23,55$; São Paulo, SP

-40,6; -19,94; Não identificada, Santa Teresa, ES

-40,07; -19,39; Regência. Reserva Biológica de Comboios, Linhares, ES

-40,75; -20,04; Não identificada, Santa Maria de Jetibá, ES

-42,14; -19,79; Estação Biológica de Caratinga. Acesso Estrada B., Caratinga, MG

-40,3; -20,17; Área de Proteção Ambiental, Mestre Álvaro; Serra, ES

-41,78; -19,75; Estrada Ipanema, Caratinga, MG

-40,65; -20,35; Domingos Martins, ES

-51,32; -30,03; Fazenda Maximiliano, BR116,Km 308; Guaíba, RS

-54,65; -27,27; Dos de Mayo, Reserva de Uso Múltiple Saltitos A. Orloff; Cainguás,

Misiones

-54,8; -26,62; El Doradito, Parque Provincial Isla Caraguatay; Montecarlo, Misiones

$-54,1 ;-26,62$; San Pedro, San Pedro, Misiones

-54,27; -26,28; Zona Paranay Guazú, Refugio Privado Timbó Gigante; Montecarlo,

Misiones

-54,17; -26,33; Cuartel Río Victoria, km 273,Ruta 14,Reserva de Uso Múltiple Cuartel

Río Victoria; Guaraní, Misiones

-53,9; -26,47; Tobuna, San Pedro, Misiones

-49,75; -21,67; Fazenda Varjao, Sao Paulo 
-42,88; -20,75; Fazenda do Paraiso, Vicosa; Minas Gerais

-40,85; -16,02; Almenara, Minas Gerais

$-41,8 ;-16,82$; Araçuai, Minas Gerais

$-43,05 ;-17,17$; Botumirim, Minas Gerais

-42,13; -19,78; Caratinga, Minas Gerais

$-40,65 ;-16,75$; Felizburgo, Minas Gerais

-41,98; -16,67; Joaima, Minas Gerais

-42,5; -19,83; Rio Matipó, Minas Gerais

$-42,7 ;-21,88$; Porto Novo, Minas Gerais

-43,13; -21,48; Rio Novo, Minas Gerais

-40,08; -15,95; Salto de Divisa, Minas Gerais

-51,57; -26,17; Bituruna, Paraná

-49,68; -25,97; Campo do Tenente, Paraná

-50,-24,82; Castro, Paraná

$-54,25 ;-24,07$; Guaíra, Paraná

-53,68; -23,38; Icaraima, Paraná

$-50,-25,42 ;$ Fazenda Santa Rita, Palmeiras; Paraná

$-53,73 ;-23,35$; Porto Camargo, Paraná

-53,5; -23,07; Querência do Norte, Paraná

-52,92; -25,57; Roça Nova, Paraná

-44,3; -23,Angra dos Reis, Rio de Janeiro

$-44,72 ;-23,22$; Parati, Rio de Janeiro

-43,17; -22,05; Três Rios, Rio de Janeiro

-50,4; -28,68; Bom Jesus, Rio Grande Do Sul

-52,92; -30,02; Cachoeira do Sul, Rio Grande Do Sul

-51,77; -29,9; General Câmara, Sapiranga; Rio Grande Do Sul

$-51,33 ;-30,12$; Guaiba, Rio Grande do Sul

$-53,5 ;-28,45$; Panambi, Rio Grande do Sul

-50,58; -29,45; São Francisco de Paula, Rio Grande do Sul

$-55,03 ;-28,42$; São Luiz Gonzaga, Rio Grande do Sul

-52,43; -29,72; Santa Cruz do Sul, Rio Grande do Sul

-51,02; -30,75; Viamão, Rio Grande do Sul

-49,52; -27,8; Bom Retiro, Santa Catarina

-52,6; -27,1; Chapecó, Santa Catarina

-49,28; -26,42; Corupá, Santa Catarina

-48,48; -27,48; Florianópolis, Santa Catarina

$-48,9 ;-25,97$; Garuva, Santa Catarina

$-49,77 ;-29, J a c i n t o ~ M a c h a d o$, Santa Catarina

-49,07; -26,48; Jaraguá do Sul, Santa Catarina

-48,83; -26,3; Joinville, Santa Catarina

$-49,95$; -29,2; Praia Grande, Santa Catarina

-49,93; -28,32; São Joaquim, Santa Catarina

-48,13; -22,78; Anhembi, São Paulo

-48,85; -24,5; Apiaí, São Paulo

-47,6; -23,43; Bacaetava (antiga Ipanema), São Paulo

-49,65; -23,42; Serra da Cantareira, São Paulo

-49,33; -24,12; Itararé, São Paulo

-49,75; -21,65; Fazenda Varjão, Lins; São Paulo

-45,85; -23,18; Matto Dentro, São Paulo

-46,28; -23,77; Paranapiacaba, São Paulo 
-45,18; -22,6; Piquete, São Paulo

-45,33; -23,83; São Sebastião, São Paulo

$-39,08 ;-16,43$; Porto Seguro, Bahia

$-39,07 ;-15,3$; Una, Bahia

$-41,13$; -20,83; Afonso Claudio, Espirito Santo

$-40,65 ;-20,03$; Chaves, Espirito Santo

$-39,75 ;-18,58$; Conceição da Barra, Espirito Santo

-40,07; -19,42; Rio Doce, Espirito Santo

-40,38; -19,98; Ibiraçú (antiga Pau-Gigante), Espirito Santo

-40,88; -19,5; Rio Mutum, Espirito Santo

-40,1; -20,55; Santa Leopoldina (antiga Porto Cachoeiro), Espirito Santo

-40,6; -19,92; Santa Teresa, Espirito Santo

-41,2; -21,23; Fazenda São José, São Domingos, Espirito Santo

$-39,85 ;-18,73$; Espirito Santo

-42,02; -20,12; Alegria Simonésia, Minas Gerais

-41,33; -18,93; Floresta Central de Minas Gerais, Minas Gerais

$-40,77 ;-17,07$; Machacalis, Minas Gerais

$-41,62 ;-19,62$; Pocrane, Minas Gerais

$-41,98 ;-19,27$; Tarumirim (Córrego do Susto), Minas Gerais

$-41,52 ;-17,88$; Teófilo Otoni, Minas Gerais

-42,98; -22,43; Teresópolis, Rio de Janeiro

$-39,93 ;-19,2$; Reserva Florestal Linhares, Espirito Santo

-53,83; -29,67; Campo de Instrução de Santa Maria, Santa Maria, Rio Grande Do Sul

$-41,82 ;-19,73$; RPPN Feliciano Miguel Abdala, Minas Gerais

-42,4; -20,59; Parque Estadual da Serra do Brigadeiro, Minas Gerais

-40,53; -19,95; Estação Biológica Santa Lúcia, Santa Teresa, Espírito Santo

-49,31; -25,33; Almirante Tamandaré, Paraná

$-48,71 ;-25,43$; Antonina, Paraná

-48,99; -24,66; Parque Estadual Lauráceas, Adrianópolis, Paraná

-49,52; -25,6; Araucária, Guajuvira; Paraná

-49,41; -25,59; Araucária, Palmital; Paraná

-49,64; -25,58; Balsa Nova, Paraná

$-49,62 ;-25,49 ;$ Balsa Nova, Bugre; Paraná

-49,72; -25,47; Balsa Nova, São Luiz do Purunã; Paraná

-49,68; -25,98; Campo do Tenente, Paraná

-49,51; -25,36; Campo Largo, Bateias; Paraná

-49,65; -25,06; Campo Largo, São Silvestre; Paraná

-49,53; -25,46; Campo Largo, Três Córregos; Paraná

-50,01; -24,79; Castro, Paraná

-53,85; -25,15; Cerro Azul, Paraná

-53,86; -22,66; Diamante do Norte, EE Caiuá; Paraná

-49,31; -25,66; Fazenda Rio Grande, Paraná

$-54,59 ;-25,55$; Foz do Iguaçu, Paraná

-48,33; -25,31; RPPN Salto Morato, Guaraqueçaba, Paraná

-48,58; -25,88; Guaratuba, Cubatão; Paraná

$-51,05 ;-23,27$; Ibiporã, Paraná

$-53,61 ;-23,4$; Icaraíma, Paraná

-53,74; -23,37; Icaraíma, Porto Camargo; Paraná

$-52,2 ;-22,75 ;$ Inaja, Paraná

-49,72; -25,77; Lapa, Paraná 
-48,83; -25,48; Morretes, Paraná

-48,88; -25,44; Morretes, PE Pico Marumbi; Paraná

-51,99; -26,48; Palmas, Paraná

$-50,01 ;-25,43$; Palmeira, Paraná

-49,95; -24,53; Piraí do Sul, Roseta; Paraná

-49,06; -25,44; Piraquara, Paraná

-50,16; -25,1; Ponta Grossa, PE Vila Velha; Paraná

-49,89; -25,55; Porto Amazonas, Paraná

-49,05; -25,39; Quatro Barras, Borda do campo; Paraná

-52,91; -25,45; Quedas do Iguaçu, Rio das Cobras; Paraná

-53,48; -23,08; Querência do Norte, Paraná

-50,3; -25,68; São João do Triunfo, Paraná

-49,21; -25,54; São José dos Pinhais, Renaut; Paraná

$-50,32 ;-25,87$; São Mateus do Sul, Paraná

-50,58; -23,91; Sapopema, Paraná

-50,62; -24,32; Telêmaco Borba, Paraná

-50,41; -24,51; Tibagi, Guartelá; Paraná

-49,2; -25,93; Tijucas do Sul, Paraná

-49,09; -24,97; Tunas do Paraná, Paraná

$-50,24 ;-24,25 ;$ Ventania, Paraná

$-53,73 ;-23,51 ;$ Vila Alta, Paraná

-41,01; -16,34; Mata Escura, Jataí, Duas Barras; Jequitinhonha, MG

-40,24; -15,36; Fazenda São Pedro, estrada entre Itapetinga e Maiquinique, próximo à

balsa do rio Pardo; BA

-51,03; -30,38; Morro do Campista, Ponta de Itapuã; Viamão, RS

$-44,58 ;-22,42$; Parque Nacional do Itatiaia, RJ

$-48,15 ;-22,67$; Barreiro Rico, SP

-43,28; -21,75; Fazenda Floresta , Juíz de Fora, MG

-46,93; -22,84; Mata Ribeirão Cachoeira, Campinas, SP

-43,9; -21,71; Mata Grande, Parque Estadual Ibitipoca; MG

-43,86; -21,71; Mata do Patuá, MG

-43,88; -21,65; Mata dos Luna, MG

$-44,61 ;-22,44$; Parque Nacional do Itatiaia, RJ

-51,05; -23,27; Mata Doralice, Ibiporã, PR

\section{Aotus azarae infulatus}

-46,36; -3,67; Reserva Indígena Caru, MA

-46,75; -1,88; Fazenda Amanda, Viseu, PA

$-50,3 ;-5,15$; Ilha de Germoplasma (Usina Hidrelétrica de Tucuruí), PA

$-57,98 ;-4,67$; área de amortecimento, PA

$-50,12 ;-10,36 ;$ TO

$-46,71 ;-3,54$; MA

$-50,54 ;-5,62 ; \mathrm{PA}$

$-49,18 ;-10,44$; TO

$-54,3 ;-2,52$; Os Patos, PA

$-54,28 ;-2,53$; Taperinha, PA

-54,4; -2,55; Maicá, PA

$-54,95 ;-2,63$; Belterra, PA 
$-55,-2,67$; Cajutuba, PA

$-55,-2,72$; Aramanaí, PA

$-55,02 ;-2,78$; Maguari, PA

$-54,97 ;-2,67$; Piquiatuba, PA

-55,13; -2,83; Caxiricatuba, PA

$-55,08 ;-2,9$; Tapaiúna, PA

$-55,03 ;-2,95$; Itapoama, PA

$-55,17 ;-3,25$; Aveiros, PA

$-55,48 ;-3,83$; FLONA Tapajós, PA

$-55,47 ;-3,83$; Araipá, PA

$-55,63 ;-4,1$; Monte Cristo, PA

-55,62; -4,05; Pedreira, Rio Tapajós; PA

-53,82; -4,55; Mundo Novo, PA

-53,-4,Largo do Souza, Rio Irirí; PA

-53,06; -3,76; Laranjal, tribo indígena arara; PA

$-54,95$; -3,36; Base 117 (Base Sucupira), PA

-54,97; -2,58; Belterra, PA

$-54,94 ;-2,64$; Iruçanga, PA

$-55,01 ;-2,78$; Caxiricatuba, PA

$-55,1 ;-3,05$; Piquiatuba, PA

$-55,05 ;-2,82$; Caxiricatuba, PA

$-49,67 ;-3,77$; banco oeste do rio Tocantins, Tucurui, PA

$-50,2 ;-4,91$; Vila Brabo, PA

-49,64; -4,07; Sítio Calandrinho, PA

$-46,14 ;-1,2$; Timbozal, PA

$-49,48 ;-4,07$; Saúde, PA

$-51,24 ;-1,03$; Cocal, PA

-49,25; -8,28; Conceição do Arraguaia, PA

-49,57; -0,93; Lago Arari, Ilha Marajó, PA

-48,87; -1,39; Ponta de Pedras, Ilha Marajó, PA

-49,91; 0,18; Fazenda Santana, Ilha Caviana, PA

-50,8; 0,71; Carmo do Macacoari, Itaubal, AP

$-56,54$; -9,05; ambas as margens do rio São Benedito, Jacareacanga, PA

-49,-3,72; Reservatório da Usina Hidrelétrica (UHE) de Tucuruí, PA

-50,-5, 25; Reservatório da Usina Hidrelétrica (UHE) de Tucuruí, PA

-49,2; -3,72; Usina Hidrelétrica de Tucuruí, PA

$-49,75 ;-4,5$; Reservatório da Usina Hidrelétrica de Tucuruí, PA

$-49,51 ;-4,27$; Reservatório da Usina Hidrelétrica de Tucuruí, PA

$-42,1 ;-3,32$; Murici das Portelas, PI

$-46,57 ;-5,47$; Jurema, Amarante, MA

$-46,13 ;-5,82$; Grajaú, MA

$-46,5 ;-4,6$; Fazenda Mapisa, Buriticupu, MA

-45,47; -3,88; Perto de Santa Luzia, rio Zutina; MA

$-45,92 ;-4,7$; Perto de Arame, MA

$-44,65 ;-2,42$; Santa Maria, Alcantara, MA

-44,72; -2,47; Canelatiua, Alcantara, MA

-44,47; -4,95; São José das Verdades, Bacabal, MA

$-44,75 ;-4,07$; Perto de Lago Verde, MA

$-44,88 ;-3,47$; Morada Nova, margem esquerda do rio Ipixua-Açu; Vitória do Mearim,

MA 
-45,-4,43; Pedra Preta, Lago da Pedra, MA

-44,78; -4,2; Rio Mearim, Bacabal, MA

$-44,57 ;-4,37$; Margem direita do Rio Mearim,

$-44,13 ;-4,13$; Perto de Coroatá,

-44,45; -4,02; Nova Guiné, São Mateus, MA

$-44,45 ;-3,67$; Palmeiral, Matões, MA

$-43,6$; -4,65; São Miguel, margem esquerda do rio Parnaíba; Caxias, MA

$-42,43 ;-4,82$; Brejinho, Caxias, MA

-46,05; -9,47; Estiva, Alto Parnaíba, MA

$-49,65 ;-3,87$; Ilha de Germoplasma (Usina Hidrelétrica de Tucuruí), PA

$-48,78 ;-2,6$; Maçaranduba, PA

\section{Aotus azarae azarae}

-57,66; -19,01; Fronteira com Bolívia, Corumbá, MS -57,65; -19,07; Maciço de Urucum, Corumbá, MS

-57,7; -19,03; Divisa Brasil-Bolívia, Corumbá, MS

-57,49; -18,04; Serra do amolar, Corumbá, MS

-57,65; -19,07; Maciço de Urucum, Corumbá, MS

-57,65; -19,07; Maciço de Urucum, Corumbá, MS

-57,65; -19,07; Maciço de Urucum, Corumbá, MS

-57,7; -19,03; Divisa Brasil-Bolívia, Corumbá, MS

-57,7; -19,03; Divisa Brasil-Bolívia, Corumbá, MS

-57,7; -19,03; Divisa Brasil-Bolívia, Corumbá, MS

-57,66; -19,01; Fronteira com Bolívia, Corumbá, MS

-57,66; -19,01; Fronteira com Bolívia, Corumbá, MS

-57,66; -19,01; Fronteira com Bolívia, Corumbá, MS

-57,49; -18,04; Serra do amolar, Corumbá, MS

$-57,65 ;-19,01$; Corumbá, MS

$-57,65 ;-19,01$; Corumbá, MS

$-57,65 ;-19,01$; Corumbá, MS

-57,65; -19,07; Maciço do Urucum, Corumbá, MS

-57,65; -19,07; Maciço do Urucum, Corumbá, MS

-57,7; -19,03; Fronteira Brasil-Bolívia, Corumbá, MS

-57,7; -19,03; Fronteira Brasil-Bolívia, Corumbá, MS

-57,7; -19,03; Fronteira Brasil-Bolívia, Corumbá, MS

-57,7; -19,03; Fronteira com Bolívia, Corumbá, MS

-57,7; -19,03; Fronteira Brasil-Bolívia, Corumbá, MS

-57,49; -18,04; Serra do Amolar, Corumbá, MS

-57,49; -18,04; Serra do Amolar, Corumbá, MS

-57,49; -18,04; Serra do Amolar, Corumbá, MS

-57,49; -18,04; Serra do amolar, Corumbá, MS

$-57,66 ;-19,01$; Corumbá, MS

-57,65; -19,01; Corumbá, MS

-57,7; -19,03; Fronteira Brasil-Bolívia, Corumbá, MS

-57,7; -19,03; Fronteira Brasil-Bolívia, Corumbá, MS

-57,49; -18,04; Corumbá, MS

-67,52; -16,41; Chulumani, La Paz - Bolívia

-63,48; -17,37; VERY CLOSE TO SANTA ROSA, Santa Cruz - Bolívia 
-57,66; -23,1; Rio Verde, Los Lapachos; Presidente Hayes - Paraguai

-67,55; -14,33; Rio Beni, Puerto Salinas; Beni - Bolivia

-59,-27, Rio de Oro, Chaco - Argentina

-65,-17,Bolivia; San Firmin,

-64,97; -14,17; Rio Tijamuchi, 240 M; 14D56'S, 65D09'W; Beni - Bolivia

$-65,-14$, Beni; Confl Madr de Dios, Victoria; Beni - Bolivia

$-63,63 ;-17,45$; Buena Vista, Ichilo, Santa Cruz

$-58,66 ;-26,7$; Estancia Velaz, Bermejo, Chaco

$-64,79 ;-23,84$; Ledesma, Ledesma, JUJUY

$-64,82 ;-13,16$; ca. 20 kilometers southwest of San Joaquin, Estancia Yotiole; Mamore,

Beni

-65,35; -13,72; Puerto Caballo, Mamore River; Yacuma, Beni

$-67,57 ;-15,65 ; 35$ kilometers by road north of Caranavi, Serrania Bella Vista; Nor

Yungas, La Paz

-47,33; -1,03; Nova Timboteua, Mata Do Bais, Timboteua Velba, Fazenda Sao

Francisco Do Trombeta, Francisco R. De Morais; Para

$-64,97 ;-14,33$; ca. 10 kilometers west of San Pedro, Yacuma, Beni

$-63,21 ;-21,2$ kilometers south, 10 kilometers east of Tiguipa, Laguna Palmar; Luis

Calvo, Chuquisaca

-69,13; -11,33; Centro Grande, Manuripi, Pando

$-67,53 ;-14,43$; Rurrenabaque, El Beni

-58,47; -15,85; Caceres, Porto Espiridiao, Rio Jauru, Sitio Eucantadinho; Mato Grosso

$-60,82 ;-26,67 ;$ Sin localidad precisa, Chaco

-58,15; -25,07; Camino a Latakue, Formosa

-58, 17; -25,12; Puente $\mathrm{E}^{\mathrm{o}}$ Poi, Formosa

$-58,17 ;-25,12$; Destacamento Estero Poí. \&Aacute; rea de acampe, Formosa

-58,18; -25,12; Destacamento Estero Poí, Formosa

$-58,21 ;-25,08$; Estero Poi, Formosa

-58,18; -25,12; Area acampe, Dto. E ${ }^{\circ}$ Poi; Formosa

-58,15; -25,07; Camino a Latakue, Formosa

$-58,02 ;-25,12$; Ex. Pto. San Miguel, Formosa

$-58,13 ;-25,17$; S. Secretos del Monte, Formosa

-58,17; -25,12; Dto. E ${ }^{\mathbf{o}}$ Poi (Detr\&aacute; s de la caballeriza), Formosa

-58,17; -25,12; Destacamento Estero Poi, en el borde del monte.; Formosa

-58,26; -24,93; Junta de Fontana, Formosa

-58,31; -24,99; Paso Piedras (cercanías de Pto. Ramos), Formosa

$-60,-22,5$; Colonia Mennonita, Orloff; Boqueron

-57,92; -22,33; Puerto Casado, 7 km SW Laguna General Diaz; Alto Paraguay

-57,97; -22,33; Puerto Casado, $30 \mathrm{~km} \mathrm{~W}$ on RR; Alto Paraguay

-65,63; -17,08; Junction of Rio San Antonio and Rio Espirito Santo, colonias; Chapare,

Cochabamba

$-65,48 ;-17,1$; Palmar, Chapare, Cochabamba

$-63,66 ;-17,46$; Buena Vista, Bolivia;

$-63,67 ;-17,45$; Buena Vista, Ichilo, Santa Cruz

-63,16; -17,79; Santa Cruz de la Sierra, Andrés Ibáñez, Santa Cruz

-69,-14,Pampa Grande, below San Ignacio; Sandia, Puno

$-64,82 ;-13,07$; San Joaquin, Mamore, El Beni

$-64,85 ;-13,02$; Azunta (=Casa Morona), Mamore, El Beni

-52,37; -3,65; Altamira, 52 Km SSW, E Bank Rio Xingu; Para

-58,18; -25,05; Puente de Estero Poí, Formosa - Argentina 
-60,8; -27,18; Sobre el camino a las lagunas, a escasos metros del triángulo de acceso al área operativa; Chaco - Argentina

$-62,02 ;-22,78$; quebrachal, Paraguai

-63,06; -17,79; Jardim Botânico de Santa Cruz, Santa Cruz - Bolívia

-58,18; -25,47; Riacho Pilagá, Argentina

$-58,22$; -26,9; Estancia Guaycoléc, Rio Pilagá; Formosa- Argentina

-58,18; -25,97; Estancia Guaycoléc, Rio Pilagá; Formosa- Argentina

$-62,62 ;-14,63$; Perseverancia, Santa Cruz- Bolivia

-62,75; -15,58; Arroyo Chuchui, Santa Cruz- Bolivia

-62,1; -14,5; San Martin, Santa Cruz- Bolivia

$-63,32 ;-15,05$; El Tutumo, Santa Cruz- Bolivia

-63,52; -14,95; Pajaral, Santa Cruz- Bolivia

\section{Callicebus donacophilus}

-57,65; -18,31; Fazenda Santa Teresa, Corumbá, MS

-57,65; -19,01; Corumbá, MS

-57,65; -19,01; Corumbá, MS

-57,65; -19,01; Corumbá, MS

-57,65; -19,07; Maciço do Urucum, Corumbá,

-57,65; -19,07; Maciço do Urucum, Corumbá, MS

-57,65; -19,07; Maciço do Urucum, Corumbá, MS

-57,65; -19,07; Maciço do Urucum, Corumbá, MS

-57,7; -19,03; Fronteira Brasil-Bolívia, Corumbá, MS

-57,7; -19,03; Fronteira Brasil-Bolívia, Corumbá, MS

-57,7; -19,03; Fronteira Brasil-Bolívia, Corumbá, MS

-57,7; -19,03; Fronteira Brasil-Bolívia, Corumbá, MS

-57,49; -18,04; Serra do Amolar, Corumbá, MS

-57,49; -18,04; Serra do Amolar, Corumbá, MS

-57,49; -18,04; Serra do Amolar, Corumbá, MS

$-59,58 ;-22,67$; Fort Wheeler, Presidente Hayes

-63,63; -17,45; Buena Vista, Ichilo, Santa Cruz

-64,24; -16,18; Suruto River, Santiesteban, Santa Cruz

$-64,88$; -15,36; ca. 12 kilometers northwest of Limoquije, Camiaco; Marban, Beni

$-66,74 ;-12,4$; ca. 10 kilometers east of San Antonio, General Jose Ballivian, Beni

-65,14; -16,69; San Antonio, Chapare, Cochabamba

$-65,13 ;-16,8$; Todos Santos, Carrasco, Cochabamba

$-57,97$; -22,33; Puerto Casado, $30 \mathrm{~km} \mathrm{~W}$ on RR; Alto Paraguay

$-63,67 ;-17,45$; Buena Vista, Ichilo, Santa Cruz

-63,17; -17,74; Santa Cruz de la Sierra, Bolivia;

$-65,13 ;-16,8$; Todos Santos, Chapare, Cochabamba

$-66,31 ;-14,84$; Perto da estación biosférica del Beni, Beni - Bolivia

$-66,23 ;-14,73$; Perto da estación biosférica del Beni, Beni - Bolivia

$-65,62 ;-14,99$; Perto da estación biosférica del Beni, Beni - Bolivia

$-65,81 ;-14,25$; Perto da estación biosférica del Beni, Beni - Bolivia

$-61,41 ;-13,49$; Rondônia

-63,06; -17,79; Jardim Botânico de Santa Cruz, Santa Cruz - Bolívia

-63,15; -17,93; Parque Regional Lomas de Arena, Santa Cruz - Bolívia

-65,86; -12,97; Coquinal, lago Rogaguado; Beni- Bolívia 
-63,72; -13,6; Huacaraje, Beni- Bolívia

-64,84; -15,33; Rio Mamoré, Beni- Bolívia

$-66,26 ;-14,75$; Beni- Bolívia

-65,63; -14,99; Lagoa Isireri, Beni- Bolívia

\section{Callicebus nigrifons}

-42,67; -20,55; Parque Estadual Serra do Brigadeiro, Serra da Mantiqueira; MG

-45,11; -23,36; Parque Estadual Serra do Brigadeiro, Serra da Mantiqueira; MG

$-42,57 ;-19,74 ; \mathrm{MG}$

-42,06; -22,24; rio Onças, Campos, RJ

$-42,85 ;-20,75$; Viçosa, MG

$-43,35 ;-21,76$; Juiz de Fora, MG

$-44,55 ;-22,42$; Trilha Maromba-Lamego, RJ

-44,62; -22,43; Trilha Maromba-Lamego, RJ

-44,58; -22,42; Trilha dos Três Picos, RJ

-44,6; -22,43; Trilha dos Três Picos, RJ

$-44,65 ;-22,33 ; \mathrm{RJ}$

-41,79; -20,43; entorno do PARNA do Caparaó, MG

$-43,9 ;-21,72 ; \mathrm{MG}$

-46,25; -21,38; Mata da Olaria na Fazenda Lagoa, Monte Belo, MG

-42,74; -20,07; Fazenda Esmeralda, Rio Casca, MG

-42,74; -20,12; Fazenda Esmeralda de Cima, Rio Casca, MG

-42,74; -20,06; Fazenda Esmeralda, Rio Casca, MG

$-42,42 ;-20,67 ; \mathrm{MG}$

$-44,75 ;-22,32 ; \mathrm{RJ}$

$-44,83 ;-22,75$; RJ

$-43,48 ;-20,3$; Parque Estadual do Itacolomi, Ouro Preto, MG

$-45,-21,25$; Fragmentos Florestais, Lavras, MG

$-47,47$; -22,37; Fazenda São José, SP

-45,97; -22,22; Parque Municipal de Pouso Alegre, Pouso Alegre, MG

-42,86; -20,8; Mata do Paraíso, Viçosa, MG

-48,1; -22,68; Fazenda Barreiro Rico, SP

-42,04; -21,47; Bairro dos Alves, Monte Alegre, SP

-48,39; -21,99; Fazenda Itaquerê, Boa Esperança do Sul, SP

$-47,48 ;-20,6$; Franca, SP

$-44,56 ;-22,5 ;$ Itatiaia, RJ

$-46,84 ;-23,01$; Itatiba, SP

-41,17; -19,39; Rio Doce, ltuéta, MG

-49,74; -21,68; Rio Tietê, Lins, SP

$-48,57$; -20,56; Mato Grosso, Barretos, SP

$-46,52 ;-18,58 ;$ Patos, MG

-42,84; -20,11; banco direito do Rio Doce, MG

-46,61; -20,72; São João da Glória, Passos, MG

-46,64; -23,55; São Paulo, SP

$-48,28 ;-18,92$; Uberlândia, MG

$-48,8 ;-20,42$; Usina HidrelÚtrica Nova Ponte, Nova Ponte, MG

-42,68; -20,14; Rio Doce, banco direito; MG

-46,51; -20,64; São João Baptista do Glória, MG 
$-43,78 ;-16,36$; Tapera, MG

$-47,4 ;-20,54 ;$ Franca, SP

$-46,43 ;-23,49 ;$ Itaquera, SP

$-43,46 ;-19,87$; Matodentro, SP

$-43,55 ;-21,85$; Torrões, Juiz de fora, MG

-44,63; -22,38; Pico das Agulhas Negras, Macieiras; Petropolis, Rio de Janeiro

-43,36; -21,74; Fazenda Tapera, Juiz de Fora, Minas Gerais

$-51583332,-20,67$; Rio Tiete, Sao Paulo

-47,47; -22,37; Fazenda São José, São Paulo

-42,85; -20,75; Universidade Federal de Viçosa, Viçosa, Minas Gerais

-44,92; -21,3; Serra do Carrapato, Lavras, Minas Gerais

$-44,58 ;-22,42$; Parque Nacional do Itatiaia, RJ

$-43,48 ;-20,08$; Serra do Caraça, MG

-46,87; -23,18; Reserva Municipal Serra do Japi, Jundiaí, SP

-46,59; -23,4; Parque Estadual da Cantareira, Serra da Cantareira; SP

-46,92; -22,83; Mata Ribeirão Cachoeira, Campinas, SP

$-48,15$; -22,67; Barreiro Rico, SP

-46,93; -23,23; Serra do Japi, Reserva Biológica Municipal de Jundiaí; Jundiaí, SP

-43,28; -21,75; Fazenda Floresta , Juíz de Fora, MG

-46,93; -22,84; Mata Ribeirão Cachoeira, Campinas, SP

$-43,9 ;-21,71$; Mata Grande, Parque Estadual Ibitipoca; MG

-43,86; -21,71; Mata do Patuá, MG

$-43,88 ;-21,65$; Mata dos Luna, MG

$-44,59 ;-22,42$; Parque Nacional do Itatiaia, RJ

-42,86; -20,8; Mata do Paraíso, Viçosa, MG

-43,98; -20,08; Estação Ecológica Fechos, Belo Horizonte, MG

\section{Callicebus personatus}

-39,05; -15,2; Lemos Maia estação experimental , Uma, BA

$-42,48 ;-20,75$; Fazenda Neblina, MG

$-42,46$; -20,68; Fazenda Neblina, MG

-42,45; -20,7; Fazenda Brigadeiro, MG

-42,42; -20,67; Fazenda Brigadeiro, MG

$-42,43 ;-20,65$; Ararica, MG

$-42,43 ;-20,64$; São Bento, MG

$-42,4 ;-20,6$; São Bento, MG

$-42,36 ;-20,64 ; \mathrm{MG}$

-42,5; -17,75; Córrego da Chacara, Minas Novas, MG

-41,8; -16,82; Fazenda Santa Clara, Vila Piauí; Araçuaí, MG

-41,93; -16,6; Fazenda Santa Maria, Itinga, MG

$-41,87 ;-16,72$; Taquaral, Itinga, MG

-41,35; -16,82; Fazenda Lagoa Enconberta, Joiama, MG

$-40,25 ;-19,08$; Linhares, ES

-39,92; -18,88; Linhares, ES

$-40,18 ;-18,32$; Pinheiros, ES

$-40,23 ;-18,52$; Pinheiros, ES

$-40,05 ;-19,32$; baixo rio Doce, ES

-40,5; -19,77; Santa Tereza, ES 
$-40,58 ;-19,92 ;$ Santa Tereza, ES

-40,48; -20,3; Reserva Florestal de Duas Bocas (RFDB), Cariacica, ES

-40,53; -20,32; Reserva Florestal de Duas Bocas (RFDB), Cariacica, ES

-41,01; -20,42; Reserva Florestal de Pedra Azul (REPA), Domingos Martins, ES

-39,87; -19,03; Reserva Florestal da Companhia Vale do Rio Doce (RCVRD), Linhares, ES

-40,1; -19,25; Reserva Florestal da Companhia Vale do Rio Doce (RCVRD), Linhares, ES

-39,76; -18,66; Conceição da Barra, ES

-41,01; -20,67; Reserva Florestal da Fazenda Montes Verdes (RFFMV), Vargem Alta, ES

-40,52; -19,97; Estação Biólogica de Santa Lúcia (EBSL), ES

-40,6; -19,94; Estação Biólogica de São Lourenço (EBSL), ES

-45,47; -22,75; Fazenda São Sebastião do Ribeirão Grande - Serra da Mantiqueira,

Pidamonhangaba, SP

-48,1; -22,68; Fazenda Barreiro Rico, SP

-48,19; -22,68; Sarã, Fazenda Barreiro Rico; SP

$-40,7 ;$-20,04; fragmento de mata em propriedades privadas, Santa Maria de Jetibá, ES

$-47,47 ;-22,37$; Fazenda São José, SP

$-42,64 ;-19,8 ; \mathrm{MG}$

$-42,47 ;-19,49 ; \mathrm{MG}$

$-40,16 ;-19, \mathrm{ES}$

$-39,75 ;-19,1 ; \mathrm{ES}$

$-39,82 ;-18,42 ; \mathrm{ES}$

$-40,15 ;-18,35 ; \mathrm{ES}$

$-39,8 ;-18,26$; ES

$-39,76 ;-19,1$; ES

$-39,92 ;-18,55$; Linhares, MG

$-39,25 ;-13,83$; Ituberá, BA

-43,28; -18,41; Baixo Rio Suaçuí, MG

-40,63; -19,54; Colatina, ES

-39,88; -19,03; Estrada Linhares-São Mateus, ES

-40,1; -19,2; Lagoa Japurá, ES

-40,63; -19,64; Rio Doce, ES

-40,14; -18,99; Sooretama, ES

$-41,51 ;-17,86$; Teófilo Otoni, MG

$-40,17 ;-19 ; \mathrm{ES}$

$-41,1 ;-20,52$; Castelo, ES

-41,13; -20,52; Castelo, ES

-41,08; -20,52; Fazenda Forno Grande, ES

$-43,55 ;-20,39$; Ouro Preto, MG

$-40,53 ;-19,95 ;$ Santa Teresa, ES

$-40,67 ;-19,63 ;$ ES

$-40,75 ;-19,77 ;$ ES

$-40,53 ;-19,97 ; \mathrm{ES}$

-40,6; -19,93; Reserva Municipal São Lourenço, ES

$-40,45 ;-19,75 ;$ ES

$-40,12 ;-19,8$; Fragmento Florestal Aracruz Cellulose, ES

$-39,78 ;-18,25 ;$ ES

$-40,15 ;-18,35 ; \mathrm{ES}$ 
-40,03; -19,2; Reserva Natural Vale do Rio Doce, ES

$-40,08 ;-18,98 ;$ ES

-40,75; -20,64; Engenheiro Reeve, ES

-40,39; -19,76; Estrada Linhares-São Mateus, ES

-43,35; -21,76; Fazenda Tapera, Juíz de Fora, ES

-40,07; -19,39; Linhares, ES

-41,86; -18,97; Rio Suaçui, Rio Doce; ES

-42,78; -21,68; São Domingos, ES

$-39,86 ;-18,72$; São Mateus, ES

$-43,55 ;-21,85$; Torróes, Juiz de fora, MG

-43,55; -21,85; Distrito de Torreóes, Juiz de Fora, MG

-47,8; -21,58; Estação Ecológia de Jataí, Luís Antônio, São Paulo

-46,87; -23,17; Serra do Japi, Jundiaí, São Paulo

-40,74; -20,06; Fragmento Florestal, Santa Maria de Jetibá, ES

-41,07; -19,9; Córrego Manteiga, Laranja da Terra, ES

-40,6; -19,92; Colégio Santa Catarina, Santa Teresa, ES

-40,17; -19,Rebio de Sooretama, Sooretama, ES

$-40,-19$, Sooretama, Sooretama, ES

$-40,47 ;-19,53$; Colatina, ES

-40,09; -18,99; Sooretama, Linhares, ES

-40,56; -19,86; ReBio Augusto Ruschi, Santa Teresa, ES

-40,16; -19,87; M7/317,Aracruz, ES

-41,02; -20,4; PE Pedra Azul, Domingos Martins, ES

$-40,13 ;-19,05$; REBIO de Sooretama, Sooretama, ES

-40,33; -19,26; Rod. ES-245 (Rio Bananal - Linhares), 10 km a L; Rio Bananal, ES

-40,6; -19,92; Santa Teresa, ES

-40,55; -19,88; REBIO Augusto Ruschi (Nova Lombardia), Santa Teresa, ES

-40,53; -19,98; ReBio de Santa Lúcia, Santa Teresa, ES

-40,05; -19,1; Reserva da CVRD, Linhares, ES

-40,59; -19,96; Santa Lúcia, Santa Teresa, ES

-40,81; -20,32; prox. de Paraju, Domingos Martins, ES

-40,68; -19,84; prox. de Santo Antônio, Santa Teresa, ES

$-40,69 ;-19,81$; prox. de São João de Petrópolis, ES

-40,12; -19,8; Fragmento de MA Aracruz Celulose, Aracruz, ES

-40,61; -19,94; São Lourenço, Santa Teresa, ES

-39,85; -18,73; Rod. BR-101 (Linhares - São Mateus), São Mateus, ES

-40,03; -19,2; Reserva da CVRD, Linhares, ES

-40,6; -19,92; Estação Biológica de São Lourenço, Santa Teresa, ES

-40,54; -19,97; Estação Biológica Santa Lúcia, Santa Teresa, ES

-40,6; -19,92; Avenida da Penha, Santa Teresa, ES

-40,16; -19,9; Putiri, Aracruz, ES

-39,7; -18,42; PE de Itaúnas, Conceição da Barra, ES

-40,65; -19,15; Faz. São José (= Mata de São José), São Domingos, ES

-40,12; -19,81; Aracruz Celulose, Aracruz, ES

-41,01; -20,41; Pedra Azul, Domingos Martins, ES

$-40,33 ;-19,27$; Rio Bananal, ES

$-40,08 ;-19,47$; prox. de Bebedouro, Linhares, ES

$-41,21$; -20,61; prox. da Sede, Castelo, ES

$-40,42 ;-19,37$; prox. de São Rafael, ES

-40,67; -20,37; Domingos Martins, Domingos Martins, ES 
-39,84; -18,26; Córrego Grande (ex- Faz.), Conceição da Barra, ES

-41,07; -20,27; prox. de Barcelos, Domingos Martins, ES

-40,14; -18,35; Córrego do Veado, Pinheiros, ES

-41,01; -20,44; Faz. Montes Verdes (= Hotel Faz. Caezar Park, 24 km a SE da Sede);

Venda Nova do Imigrante, ES

-40,62; -19,12; Faz. Braço do Sul (= Afluente Branco do Sul), São Domingos, ES

-39,75; -18,58; Faz. Klabin (= Faz. São Joaquim), 1 km ao $S$ de Itaúnas; Conceição da

Barra, ES

-39,92; -19,68; Comboios (e AI Comboios), Linhares, ES

$-41,11 ;-20,51$; Forno Grande, Castelo, ES

$-41,1 ;-20,52$; Parque Estadual do Forno Grande, Castelo, ES

-40,66; -20,38; prox. da Sede, Domingos Martins, ES

-41,48; -20,78; Engenheiro Reeve (= Rive), Alegre, ES

$-40,38 ;-19,75$; prox. da Sede, João Neiva, ES

-40,64; -19,14; Faz. Dez de Agosto, São Domingos, ES

-39,95; -19,12; Linhares - CVRD, Linhares, ES

$-40,5 ;-20,3$; Duas Bocas, Cariacica, ES

-40,55; -19,85; Augusto Ruschi (ex- Nova Lombardia), Santa Teresa, ES

$-40,33 ;-19,27$; prox. da Sede, Rio Bananal, ES

-40,07; -19,34; Lagoa Juparanã, Santa Ana; Linhares, ES

-47,88; -22,36; seguindo pela estrada da cachoeira do Saltão, próximo ao início de

descida da cuesta; Itirapina, São Paulo

-48,13; -22,05; Ribeirão Bonito, São Paulo

$-48,2$; -22,12; Ribeirão Bonito, São Paulo

$-48,02 ;-22,1$; Brotas, São Paulo

-48,18; -22,65; Anhembi, São Paulo

-48,12; -22,65; Botucatu, São Paulo

-46,73; -21,6; Divinolândia, São Paulo

-46,7; -21,72; São Sebastião da Grama, São Paulo

$-48,23$; -22,25; Brotas, São Paulo

$-46,35 ;-23,27$; Mairiporã, São Paulo

-46,98; -23,28; Cabreúva, São Paulo

$-46,88 ;-23,2$; Jundiaí, São Paulo

$-48,84$; -23,01; Itatiba, São Paulo

-46,68; -22,68; Monte Alegre do Sul, São Paulo

-40,6; -19,94; Colégio Santa Catarina, Santa Teresa, Espírito Santo

-41,06; -19,9; Córrego Manteiga, Laranja da Terra, Espírito Santo

-40,65; -20,35; Domingos Martins, Espírito Santo

-40,75; -20,04; Estrada para Recreio, Santa Maria de Jetibá, Espírito Santo

-46,79; -22,88; Morungaba, São Paulo

-46,76; -22,7; Estrada Amparo/Serra Negra, Amparo, São Paulo

-45,-21,25; Reserva Biológica do Poço Bonito, Lavras, Minas Gerais

-48,15; -20,67; Fazenda Barreiro Rico, Anhembi, São Paulo

-40,07; -19,39; Reserva Florestal de Linhares, Linhares, Espírito Santo

-48,15; -20,67; Fazenda Barreiro Rico, Anhembi, São Paulo

-39,07; -15,27; Faz Jueirana, Una, Bahia

-47,17; -22,25; Fazenda Campininha, Mogi Guaçu, São Paulo

-44,64; -22,36; 0 km from: Reserva Florestal Sooretama,

-41,18; -20,55; San Domingos, Fazenda Corrego Da Barada, Mata Corrego Da Barada;

Espirito Santo 
$-42,43 ;-21,13$; Sao Joao Da Gloria, Minas Gerais

$-19,63 ;-43,89$; Lagoa Santa,

$-19,07 ;-39,95$;

-40,04; -18,99; Reserva Biológica de Sooretama, Espirito Santo

-39,93; -19,2; Reserva Florestal Linhares, Espirito Santo

-39,85; -18,39; Floresta Nacional Rio Preto, Espirito Santo

-44,07; -19,93; Parque Estadual Fernão Dias, Minas Gerais

-42,4; -20,59; Parque Estadual da Serra do Brigadeiro, Minas Gerais

-40,53; -19,95; Estação Biológica Santa Lúcia, Santa Teresa, Espírito Santo

\section{Callithrix geoffroy}

$-43,52 ;-19,26$; Morro do Pilar, MG

$-42,58 ;-19,68 ; \mathrm{MG}$

$-43,5$; $-18,1$; Emendas, Rio Jequitinhonha; Diamantina, MG

-43,25; -18,03; São Gonçalo do Rio Preto, Diamantina, MG

-42,5; -17,75; Corrégo da Chacara, MG

-42,53; -17,1; Rio Capivarí, Chapada do Norte; Chapada do Norte, MG

-41,88; -16,72; Fazenda Arqueana, Rio Piauí; Araçuaí, MG

-41,87; -16,72; Taquaral, Araçuaí, MG

-41,35; -16,82; Fazenda Lagoa Encoberta, Joiama, MG

-41,98; -16,67; Fazenda Anta Podre, Joiama, MG

-40,67; -16,82; Fazenda Nossa Senhora das Graças, Joiama, MG

-40,68; -16,23; Almenara, Almenara, MG

$-39,93 ;-16,08$; Salto de Divisa, Salto de Divisa, MG

$-40,25 ;-19,08$; Linhares, ES

$-39,92 ;-18,88$; Linhares, ES

$-40,14 ;-18,34$; Pinheiros, ES

$-40,23 ;-18,52$; Pinheiros, ES

-40,5; -19,77; Santa Tereza, ES

-40,58; -19,92; Santa Tereza, ES

-40,48; -20,3; Cariacica, ES

$-40,53 ;-20,32$; Cariacica, ES

-39,87; -19,03; Linhares, ES

-40,1; -19,25; Linhares, ES

$-39,81 ;-18,26$; Conceição da Barra, ES

$-40,6 ;-19,93$; ES

$-40,5 ;-20,25 ; \mathrm{ES}$

-40,53; -19,95; Estação Biológica Santa Lúcia, ES

-40,55; -20,07; Represa Suiça, ES

-40,53; -20,05; Cachoeira Véu de Noiva, ES

-40,67; -20,37; Domingos Martins, ES

-41,78; -20,46; entorno do PARNA do Caparaó, MG

-42,12; -20,25; Fragmento Realeza 1,Manhuaçu, MG

-39,98; -16,3; Alto Cariri, Salto da Divisa, MG

-39,29; -16,5; Estação Vera Cruz, Porto Seguro, BA

-39,28; -16,47; Estação Vera Cruz, Porto Seguro, BA

-39,32; -16,48; Estação Vera Cruz, Porto Seguro, BA

$-43,45 ;-19,2 ; \mathrm{MG}$ 
$-43,63 ;-19,57 ; \mathrm{MG}$

$-40,12 ;-19,8$; Aracruz Celulose SA., ES

-40,66; -19,91; Museu de Biologia Mello Leitão, ES

-40,61; -19,93; Museu de Biologia Mello Leitão, ES

-40,6; -19,91; Museu de Biologia Mello Leitão, Santa Tereza, ES

-42,92; -22,49; Centro de Primatologia do Rio de Janeiro, RJ

-40,6; -19,87; Campus Ecológico da Universidade Federal de Minas Gerais, MG

-42,98; -22,49; Centro de Primatologia do Rio de Janeiro, RJ

$-39,81 ;-18,27$; Florianópolis, SC

-43,94; -19,91; Instituto de Ciências Biológicas da Universidade Federal de Minas

Gerais, MG

$-40,17 ;-19 ; \mathrm{ES}$

$-42,58 ;-19,71 ; \mathrm{MG}$

-40,7; -20,04; fragmento de mata em propriedades privadas, Santa Maria de Jetibá, ES

$-40,72 ;-17,04 ;$ Machacalis, MG

-41,88; -18,77; Baixo Rio Suaçui, Rio Doce; MG

-42,54; -19,6; Baixo Rio Piracicaba, Rio Doce; MG

$-43,45 ;-19,01$; Conceição do Mato Dentro, MG

$-41,52 ;-17,82 ;$ Teófilo Otoni, MG

-43,05; -19,75; São José da Lagoa, MG

-40,38; -19,81; Ibiraçu, ES

-40,34; -20,32; Vitória, ES

$-40,36 ;-20,32$; Argolas, ES

$-40,63 ;-19,51$; Rio Mutum, ES

-40,06; -19,37; Fazenda Jacaré, Linhares, ES

-39,93; -19,01; Estrada Linhares-São Mateus, entre km 12 e km 18; ES

$-40,65 ;-19,54$; Colatina, ES

$-42,89 ;-20,23$; Rio Doce, ES

$-43,55 ;-19,4 ; \mathrm{MG}$

$-39,23 ;-16,88 ; \mathrm{BA}$

$-39,79 ;-18,26 ; \mathrm{ES}$

$-40,14 ;-18,34 ; \mathrm{ES}$

$-40,16 ;-19, \mathrm{ES}$

$-39,89 ;-19,67 ; \mathrm{ES}$

$-40,49 ;-20,28 ; \mathrm{ES}$

$-39,77 ;-19,09 ; \mathrm{ES}$

$-39,06 ;-16,45 ; \mathrm{BA}$

$-40,6 ;-19,85 ;$ ES

$-39,26 ;-16,51 ; \mathrm{BA}$

$-38,9 ;-15,87$; BA

$-40,09 ;-19,13$; ES

-41,79; -16,31; Fazenda Córrego de Areia (Reserva), MG

-40,56; -19,86; Augusto Ruschi (ex- Nova Lombardia), Santa Teresa, ES

-40,15; -20,Estação de Biologia Marinha de Santa Cruz, Aracruz, ES

-40,44; -20,61; PE Paulo César Vinha, Guarapari, ES

$-40,47 ;-19,53$; Colatina, ES

-39,84; -18,25; Córrego Grande (ex- Faz.), Conceição da Barra, ES

-41,66; -20,38; Domingos Martins, Domingos Martins, ES

-40,53; -19,98; Santa Lúcia - Rod. Josil Espíndula - km20,Santa Teresa, ES

-40,16; -19,9; Aracruz, ES 
-40,6; -19,92; Santa Teresa, Santa Teresa, ES

-40,14; -18,35; Córrego do Veado, Pinheiros, ES

-40,61; -19,54; Rio Pancas, Colatina, ES

-40,16; -19,87; Mata da Aracruz Celulose SA., Aracruz, ES

-40,07; -19,42; Brejo Grande, Linhares, ES

-40,05; -19,1; Linhares, ES

$-40,12 ;-19,8 ;$ Aracruz, ES

-39,92; -19,68; Comboios (e AI Comboios), Linhares, ES

$-40,13 ;-19,05$; Sooretama, ES

$-40,62 ;-19,53$; Colatina, ES

-39,81; -18,29; Conceição da Barra, ES

-40,38; -19,91; Três Barras, Fundão, ES

-40,27; -19,82; Aracruz Celulose, Aracruz, ES

-40,13; -19,79; Aracruz - Área 021 - Talhão 008/009,Aracruz, ES

$-40,54 ;-19,98 ;$ Santa Teresa, ES

-40,53; -20,05; Cachoeira Véu de Noiva, Santa Leopoldina, ES

-40,03; -19,2; Reserva da CVRD, Linhares, ES

-39,85; -18,73; São Mateus, São Mateus, ES

$-40,15 ;-18,33$; Pinheiros, ES

$-40,08 ;-19,45$; Goitacazes, ES

$-39,82 ;-19,62 ;$ R. Doce (a), Colatina, ES

-39,83; -18,63; R. São Mateus, São Mateus, ES

-40,35; -20,33; Morro da Argola, Vitória, ES

$-40,07 ;-19,42$; Linhares, ES

-40,07; -19,3; Rod. BR-101 (Vitória - Salvador), km 12-18 do trecho local (Linhares -

São Mateus); Linhares ?, ES

-40,66; -19,16; Faz. São José (= Mata de São José), São Domingos, ES

$-40,2 ;-20,05 ;$ R. Reis Magos, ES

-40,5; -20,47; R. Jucú (= R. Jacú, = R. Espírito Santo), Faz. Coroaba, Araçatiba; Viana

$?, \mathrm{ES}$

-40,37; -19,84; Pau Gigante, Ibiraçu (antiga Pau Gigante), ES

-40,05; -19,38; Faz. Jacaré, Linhares, ES

$-39,85 ;-18,73$; prox. de Guriri, São Mateus ?, ES

-40,62; -19,12; Faz. Braço do Sul (= Afluente Branco do Sul), São Domingos, ES

-40,09; -18,99; Sooretama, Linhares, ES

$-39,78 ;-19,15$; R. Barra Seca, ES

$-40,65 ;-20,37$; prox. da Sede, Domingos Martins, ES

-40,59; -19,96; Santa Lúcia, Santa Teresa, ES

-40,5; -20,47; R. Jucú (= R. Jacú, = R. Espírito Santo), R. Araçatiba, Araçatiba; Viana ?,

ES

-39,96; -19,12; Linhares - CVRD, Linhares, ES

-40,07; -19,34; Lagoa Juparanã, Linhares, ES

-40,42; -19,37; prox. de São Rafael, ES

$-40,51 ;-20,31$; Duas Bocas, Cariacica, ES

$-40,33 ;-20,4 ;$ R. Jucú (= R. Jacú, = R. Espírito Santo), m. N; Vila Velha, ES

-40,64; -19,14; Faz. Dez de Agosto, São Domingos, ES

-39,76; -18,6; Itaúnas, Conceição da Barra, ES

-39,83; -19,42; Povoação, Linhares, ES

$-40,3 ;-20,28$; Mata da Ecologia da UFES, Vitória, ES

-40,33; -20,41; Jacarenema, Vila Velha, ES 
-39,75; -18,58; Faz. Klabin (= Faz. São Joaquim), Conceição da Barra, ES

$-40,63 ;-19,53 ;$ R. Doce (b), Colatina, ES

-40,88; -19,5; Foz do R. Mutum (= Murutu ?), Baixo Guandu ?, ES

-40,55; -20,07; Represa Suiça, Santa Leopoldina, ES

-41,03; -19,05; Alto Rio Novo, ES

$-40,3 ;-20,12$; Serra da Mula, Serra (?), ES

-40,48; -20,27; Tilha da Eduação Ambiental - ReBio de Duas Bocas., Cariacica, ES

-40,42; -20,58; Paulo César Vinha, Guarapari, ES

-40,38; -20,13; Mestre Álvaro, Serra, ES

-40,28; -20,33; Vila Velha do Espírito Santo, Vila Velha, ES

-39,7; -18,42; Itaúnas (= R. Itaúnas), Itaúnas, ES

$-40,6 ;-19,92 ;$ Santa Teresa, ES

-40,6; -19,94; Museu de Biologia Mello Leitão. Eencontrado morto sendo comido por

formigas., Santa Teresa, Espírito Santo

-40,34; -20,32; Em cativeiro., Vitória, Espírito Santo

-40,27; -19,82; Estação de Biologia Marinha de Santa Cruz, Aracruz, Espírito Santo

-40,07; -19,39; Brejo Grande, Linhares, Espírito Santo

-40,63; -19,54; Rio Pancas. Na mata., Colatina, Espírito Santo

-40,46; -20,38; Ribeira, Viana, Espírito Santo

-40,35; -20,35; Parque Estadual da Fonte Grande, Vitória, Espírito Santo

-40,42; -20,25; Cariacica, Espírito Santo

$-40,44 ;$-20,38; Ribeira, Viana, Espírito Santo

$-40,33$; -20,3; Parque Estadual da Fonte Grande, Vitória, Espírito Santo

-40,16; -19,95; Rodovia ES - 010,próximo à Base Oceanográfica de Santa Cruz;

Aracruz, Espírito Santo

-40,07; -19,13; Reserva Florestal da Companhia Vale do Rio Doce-CVRD, Linhares,

Espírito Santo

-38,99; -15,42; Reserva Zoobotânica-Comissão Executiva de Planejamento da Lavoura

Cacaueira-CEPLAC, Itabuna, Bahia

-40,49; -20,27; Reserva Biológica Duas Bocas, Cariacica, Espírito Santo

-40,07; -19,38; Reserva Florestal de Linhares, Linhares, Espírito Santo

-39,86; -18,72; No asfalto., São Mateus, Espírito Santo

-41,02; -20,53; Córrego do Ouro, Colatina, Espírito Santo

-40,63; -19,54; Rodovia Gether Lopes de Farias, Bairro Carlos Germano Naumann.;

Colatina, Espírito Santo

-40,29; -20,33; Barra do Jucu, Vila Velha, Espírito Santo

-40,41; -19,93; Três Barras, Fundão, Espírito Santo

-40,31; -20,28; Planetário, Universidade Federal do Espírito Santo, Goiabeiras; Vitória,

Espírito Santo

-40,37; -19,83; Pau Gigante, Colonia De Basilio Pignoton; Espírito Santo

-40,6; -19,92; Santa Teresa, Espirito Santo

-40,04; -18,99; Reserva Biológica de Sooretama, Espírito Santo

$-39,93 ;-19,2$; Reserva Florestal Linhares, Espírito Santo

-40,15; -18,34; Reserva Biológica Córrego do Veado, Espírito Santo

-39,85; -18,39; Floresta Nacional Rio Preto, Espírito Santo

-39,8; -18,27; Reserva Biológica Córrego Grande, Espírito Santo

-40,53; -19,95; Estação Biológica Santa Lúcia, Santa Teresa, Espírito Santo

-43,52; -19,26; Parque Nacional Serra do Cipó, Morro do Pilar, MG

$-42,07 ;-16,87$; Araçaui, MG

-40,75; -17,08; Machacalis, Rio Stanhem; MG 
$-41,5 ;-17,85$; Teofilo Otoni, MG

-43,42; -19,02; Conceição do Mato Dentro, MG

-43,05; -19,75; São José da Lagoa, Rio Doce; MG

$-42,52 ;-19,5$; Rio Doce, MG

-43,17; -19,92; Rio Piracicaba, MG

-42,83; -21,02; Visconde do Rio Branco, MG

$-40,07 ;-19,42$; Linhares, ES

-40,07; -19,33; Lagoa Japurana, ES

$-39,82 ;-19,62$; Rio Doce, ES

-40,62; -19,53; Colatina, Rio Doce; ES

-40,37; -19,83; Pau Gigante, ES

-40,6; -19,92; Santa Teresa, ES

-40,88; -19,5; Mutum, ES

-40,35; -20,32; Vitória, ES

-40,28; -20,33; Espírito Santo, ES

-40,33; -20,4; Rio Jacú, ES

-40,5; -20,47; Araçatiba, Rio Espirito Santo; ES

-43,74; -18,36; Barão de Guaicuhy, Diamantina, MG

$-43,77 ;-18,43$; Gouveia, MG

-43,69; -18,45; Fazenda São Roberto , Gouveia, MG

\section{Callithrix jacchus}

$-35,3 ;-5,73$; Extremós, RN

$-40,9 ;-3,83 ; \mathrm{CE}$

$-38,4 ;-12,93$; Parque Metropolitano de Pituaçu, Salvador, BA

-38,49; -3,69; Campus do Pici da Universidade Federal do Ceará, Fortaleza, CE

-34,83; -7,12; Reserva Ecológica do Depertamento de Sistemática e Ecologia, Campus

Universitário da UFPB; João Pessoa, PB

-35,2; -6,09; Estação de Experimentação do IBAMA, Nísia Floresta, RN

-35,2; -6,09; Estação de Experimentação do IBAMA, Nísia Floresta, RN

-35,09; -8,04; São Lourenço da Mata, PE

$-40,05 ;-7,21$; Araripe, CE

-34,94; -7,92; Parque Zoobotânico do Curado, Recife, PE

$-34,88 ;-8,04$; Recife, PE

-34,87; -8,02; Campus da Universidade Federal Rural de Pernambuco, Recife, PE

-35,25; -5,89; Estação Florestal e de Experimentação do IBAMA, Nísia Floresta, PB

-35,3; -5,83; Estação Florestal e de Experimentação do IBAMA, Nísia Floresta, PB

-35,2; -6,09; Estação Florestal e de Experimentação do IBAMA, Nísia Floresta, PB

-35,2; -6,08; Estação Florestal e de Experimentação - EFLEX/IBAMA, Nísia Floresta, $\mathrm{RN}$

-35,2; -6,1; Estação Florestal e de Experimentação - EFLEX/IBAMA, Nísia Floresta, $\mathrm{RN}$

-35,21; -6,09; Estação Florestal e de Experimentação - EFLEX/IBAMA, Nísia Floresta, RN

$-34,98 ;-8,02$; Camaragibe, PE

$-35,-8,01$; Camaragibe, PE

$-35,-8$, Camaragibe, $\mathrm{PE}$

-35,35; -5,85; Escola Agrícola de Jundiaí, Macaíba, RN 
-35,38; -5,88; Escola Agrícola de Jundiaí, Macaíba, RN

-34,92; -8,12; Estação Ecológica do Tapacurá, São Lourenço da Mata, PE

$-35,19 ;-5,82$; Natal, RN

$-35,3 ;-5,73$; Natal, RN

-35,36; -5,78; Casa no bairro de Mãe Luiza, Natal, RN

$-35,2 ;-5,73 ; 16^{\circ}$ Batalhão - Tirol, Natal, RN

$-35,2 ;-5,78 ; 7^{\circ}$ Batalhão de Engenharia e Combate - Nova Descoberta, Natal, RN

$-35,19 ;-5,82$; Bosque dos Namorados, Natal, RN

$-36,87 ;-5,52 ;$ Açu, RN

$-35,-8,12$; Mata do Camocim, São Lourenço da Mata, PE

$-35,2 ;-5,8$; Natal, RN

$-36,92 ;-5,56$; Natal, RN

$-36,97 ;-5,47$; Açu, RN

$-36,97 ;-5,36$; Açu, RN

$-36,49 ;-8,91$; Universidade Federal Rural de Pernambuco, PE

-34,88; -8,02; Universidade Federal Rural de Pernambuco, PE

$-34,85$; -8,02; Universidade Federal Rural de Pernambuco, PE

$-35,02 ;-7,96 ;$ PE

$-35,1 ;-8,34$; Ipojuca, PE

$-35,02 ;-8,53$; Ipojuca, PE

$-35,04 ;-8,02$; Camaragibe, PE

$-34,99 ;-8,02$; Camaragibe, $\mathrm{PE}$

-34,99; -8,02; Universidade Federal Rural de Pernambuco, PE

-34,89; -7,97; Universidade Federal Rural de Pernambuco, PE

$-34,88 ;-8,03$; PE

-35,2; -6,09; Estação Experimentação - IBAMA, Nísia Floresta, RN

$-38,54$; -3,64; Campus do Pici da Universidade Federtal do Ceará, Fortaleza, CE

$-35,2 ;-8,05 ; \mathrm{PE}$

$-35,02 ;-8, \mathrm{PE}$

$-35,19 ;-8,06 ; \mathrm{PE}$

$-35,21 ;-6,08$; Nísia Floresta, RN

$-35,2 ;-6,09$; Nísia Floresta, RN

$-35,2 ;-5,83$; Nísia Floresta, RN

$-35,25 ;-5,78$; Nísia Floresta, RN

$-35,2 ;-5,82 ; \mathrm{RN}$

$-35,2 ;-8,05 ; \mathrm{PE}$

$-35,22 ;-8,05 ; \mathrm{PE}$

$-34,99 ;-8,13 ; \mathrm{PE}$

-34,92; -7,84; Refúgio Ecológico Charles Darwin, Igarassu, PE

$-34,98 ;-8,02$; Aldeia, Camaragibe, PE

-34,87; -8,02; Universidade Federal Rural de Pernambuco, PE

-34,83; -7,92; Universidade Federal Rural de Pernambuco, PE

-35,19; -6,09; Estação de Experimentação do IBAMA, Nísia Floresta, RN

$-40,05 ;-2,91$; Acara. , CE

$-40,84 ;-2,91$; Camocim, CE

$-35,2 ;-8,07 ; \mathrm{PE}$

-35,2; -6,08; Estação de Experimentação do IBAMA, Nísia Floresta, RN

-35,3; -5,68; Estação de Experimentação do IBAMA, Nísia Floresta, RN

$-35,45 ;-7,71$; Limoeiro, PE

$-38,7 ;$-3,59; Campus Universitário do Pici, Fortaleza, CE 
-35,22; -6,09; Estação de Experimentação do IBAMA, Nísia Floresta, RN -35,2; -6,1; Estação de Experimentação do IBAMA, Nísia Floresta, RN -35,25; -5,68; Estação de Experimentação do IBAMA, Nísia Floresta, RN -35,21; -6,09; Estação de Experimentação do IBAMA, Nísia Floresta, RN -35,2; -6,09; Estação de Experimentação do IBAMA, Nísia Floresta, RN $-35,21 ;-8,05 ; \mathrm{PE}$

$-35,21 ;-8,06 ; \mathrm{PE}$

$-35,22 ;-8,05 ; \mathrm{PE}$

$-35,21 ;-8,06$; São Lourenço da Mata, PE

$-35,2 ;-8,06$; Mata do Alto da Buchada, PE

-35,2; -6,09; Estação de Experimentação do IBAMA, RN

-35,36; -5,83; Estação de Experimentação do IBAMA, Nísia Floresta, RN

-35,36; -5,68; Estação de Experimentação do IBAMA, Nísia Floresta, RN

$-35,21 ;-8,05$; Mato do Alto da Buchada, São Lourenço da Mata, PE

$-41,05 ;-4,08 ;$ PI

$-41,75 ;-4,15 ;$ PI

$-35,21 ;-8,05 ; \mathrm{PE}$

$-34,87 ;-8,02$; Floresta Dois Irmãos, PE

$-34,87 ;-8,02$; Floresta Dois Irmãos, PE

-34,88; -8,03; Horto Zoo-Botânico de Dois Irmãos, Recife, PE

$-34,99 ;-8,23 ; \mathrm{PE}$

$-34,94 ;-8,13 ; \mathrm{PE}$

$-35,21 ;-8,05 ; \mathrm{PE}$

$-35,21 ;-8,05 ; \mathrm{PE}$

$-38,96 ;-12,2$; Feira de Santana, BA

-34,91; -7,27; Campus da Universidade Federal da Paraíba, PB

-36,95; -5,53; Açu, RN

$-41,77 ;-6,38$; Valença, PI

$-38,88 ;-4,33$; BaturitÚ, CE

-39,42; -7,34; Arajara, CE

-39,72; -3,1; Icaraí, Mosquito; CE

$-38,99 ;-4,22$; Pacoti, CE

$-38,99 ;-4,3$; Guaramiranga, CE

$-38,99 ;-4,14$; Pacoti, CE

$-39,41 ;-7,23$; Crato, CE

$-38,95 ;-4,14$; Pernambuquinho, CE

-40,84; -3,99; Bom Jardim, São Benedito, CE

-40,8; -4,07; Barra, São Benedito, CE

-38,91; -4,72; Juá, CE

$-38,61 ;-6,53$; Triunfo, PB

$-37,98 ;-7,72$; Princesa Isabel, PB

$-34,97 ;-6,59$; Camaratuba, PB

$-35,15 ;-6,87$; Mamamguape, PB

$-38,02 ;-6,97$; Coremas, PB

$-38,06 ;-8,48$; Tapera, PE

-35,39; -7,64; Água Azul, Vicência, PE

-36,02; -8,31; Sítio Vertentes, Serra Vertentes; Caruarú, PE

-35,31; -8,15; São João dos Pompos, PE

-35,57; -8,69; Água Preta, PE

-36,44; -9,32; Quebrângulo, AL 
-36,13; -9,82; São Miguel dos Campos, AL

-35,78; -9,67; Usina Sinimbú, Mangabeiras; AL

-41,98; -11,55; Ibipetuba, BA

-38,68; -12,74; Corupeba, Recôncavo; BA

$-38,51 ;-12,97$; Salvador, BA

$-38,63 ;-12,75$; Ilha Madre de Deus, BA

$-38,87 ;-12,48$; Sítio Vila dos Coqueiros, BA

$-42,08 ;-12,33$; Narandiba, BA

-38,87; -11,83; Lamarãozinho, BA

-39,71; -12,15; Mata da Caboranga, BA

-39,73; -11,23; Queimada da onça, BA

-39,25; -12,15; Fazenda Campo Alegre, Anguera, BA

$-41,72 ;-4,09$; PI

$-42,57 ;-8,71 ;$ PI

$-40,9 ;-3,83 ; \mathrm{CE}$

$-38,02 ;-8,66 ; \mathrm{PE}$

$-35,18 ;-8,72 ; \mathrm{PE}$

$-36,41 ;-9,22 ; \mathrm{AL}$

$-35,17 ;-6,72 ; \mathrm{PB}$

$-34,94 ;-6,75 ; \mathrm{PB}$

$-37,3 ;-6,64 ; \mathrm{RN}$

$-37,28 ;-10,74 ; \mathrm{SE}$

$-45,16 ;-8,9 ;$ PI

$-40,13 ;-6,58 ; \mathrm{CE}$

$-36,43 ;-10,39 ; \mathrm{AL}$

$-38,64 ;-9,73 ;$ BA

$-34,8 ;-7,16 ; \mathrm{PB}$

$-38,99 ;-4,27 ; \mathrm{CE}$

$-35,19 ;-5,82 ; \mathrm{RN}$

$-34,86 ;-7,15 ; \mathrm{PB}$

$-35,25 ;-8,03 ; \mathrm{PE}$

-35,22; -7,1; Sapé - Mata de Capatuba, Sapé, Paraíba

-41,33; -12,8; Macaco Seco, near Mt Andarahy; Bahia

-38,72; -12,53; Santo Amaro, Bahia

$-44,53$; -11,Rio Preto; Santa Rita de Cassia, Bahia State

$-43,65 ;-10,72$; Mum. de Barra; Buritirama, Bahia State

$-35,84 ;-8,98$; Alagoas

$-39,3 ;-6,37$; Jua, near Iguatu; Ceara

$-40,7 ;-4,33$; Ipu, Ceara

$-40,92 ;-4,83$; Arara, Piaui

$-41,6 ;-3,65$; Deserto, Piaui

$-37,23 ;-11,2$; Fazenda Trapsa, Sergipe

-35,19; -6,09; Floresta Nacional de Nísia Floresta, Rio Grande do Norte

-42,49; -8,78; Parque Nacional da Serra da Capivara, Piaui

-37,41; -10,04; Fazenda São Pedro, Porto da Folha , SE

$-43,7 ;-6,5 ;$ São João dos Patos, MA

$-41,58 ;-8,65$; Arara, Piaui

$-40,7 ;-8,1$; Deserto, Piaui

$-39,63 ;-3,05$; Icaraí, Ceará

-38,58; -3,8; Mondubim, Ceará 
-40,88; -4,05; São Benedito, Ceará

-40,7; -4,33; Ipu, Serra do Ibiapaba; Ceará

$-38,93 ;-4,22$; Pacoti, Ceará

$-38,95 ;-4,23$; Pernambuquinho, Ceará

$-38,93$; $-4,23$; Serra de Guaramiranga, Ceará

$-39,-4,3$; Mulungu, Ceará

$-38,88 ;-4,33$; Baturité, Ceará

-40,-7,33; Chapada do Araripe, Ceará

$-39,38 ;-7,23$; Crato, Ceará

$-39,3 ;-6,37$; Iguatu, Ceará

$-35,12 ;-6,83$; Mamaguape, Paraíba

$-34,8 ;-7,17$; Penha, Paraíba

-37,97; -7,02; Coremas, Paraíba

-38,08; -8,4; Tapera, Pernambuco

$-38,12 ;-7,83$; Triunfo, Pernambuco

$-35,05$; -8,São Lourenço, Pernambuco

$-34,9$; -8,05; Dois Irmãos, Recife; Pernambuco

-36,48; -9,33; Quebrangulo, Alagoas

-36,08; -9,78; São Miguel, Alagoas

-35,72; -9,67; Maceió, Alagoas

$-36,13 ;-9,95$; Mangabeiras, Alagoas

-44,53; -11,Santa Rita de Cássia, Bahia

-38,72; -12,53; Santo Amaro, Bahia

$-38,52 ;-12,98$; Salvador, Bahia

$-38,62 ;-12,73$; Madre de Deus, Bahia

$-38,6 ;-12,72$; Curupeba, Reconcavo; Bahia

$-38,7 ;-13$, Ilha de Itaparica, Bahia

\section{Callithrix penicillata}

-47,27; -16,19; Aproveitamento Hidrelétrico de Queimado, MG

$-42,58 ;-19,73 ; \mathrm{MG}$

-42,77; -17,13; Acauá State Reserve (Instituto Estadual de Floresta), Turmalina, MG

-43,22; -17,13; Botumirim, Botumirim, MG

$-43,05 ;-17,17$; Fazenda Guajovira, Botumirim, MG

-42,63; -16,85; Fazenda Irmãos Atachi, Berilo, MG

-42,68; -16,82; Cristália, MG

-42,87; -16,75; Córrego Contendas, Cristália, MG

-42,86; -16,8; Morro do Chapeu, Cristália, MG

-42,7; -16,75; Barra do Itacambiricú, Cristália, MG

-43,2; -16,57; Fazenda Mandasaia, Grão Mogol, MG

-43,03; -16,57; Fazenda Giro, Grão Mogol, MG

-42,22; -16,7; Porto Mandacarú, Virgem da Lapa, MG

-42,22; -16,83; Fazenda Paiol, Virgem da Lapa, MG

-41,98; -16,67; Fazenda Santana, Virgem da Lapa, MG

-41,93; -16,6; Fazenda Santa Maria, Itinga, MG

$-41,78 ;-16,57$; Itinga, Itinga, $\mathrm{MG}$

$-42,64 ;-19,8 ; \mathrm{MG}$

$-42,47 ;-19,49 ; \mathrm{MG}$ 
$-43,35 ;-21,76$; Juiz de Fora, MG

-41,78; -20,42; entorno do PARNA do Caparaó, MG

-42,03; -20,24; Fragmento AABB, Manhuaçu, MG

-42,01; -20,23; Fragmento Feijoal 1,Manhuaçu, MG

-42,02; -20,28; Fragmento Pouso Alegre, Manhuaçu, MG

-43,94; -19,92; Instituto de Ciências Biológicas da Universidade Federal de Minas

Gerais, MG

$-47,99 ;-15,68 ; \mathrm{DF}$

$-52,87 ;-18,12 ; \mathrm{GO}$

$-47,63 ;-14,05 ; \mathrm{GO}$

$-46,48 ;-20,35 ; \mathrm{MG}$

$-43,89 ;-21,7$; Mata dos Luna, MG

$-42,02 ;-22,41 ; \mathrm{RJ}$

-46,5; -16,5; Centro de Primatologia da Universidade de Brasília, Fazenda Água Limpa;

$\mathrm{DF}$

$-43,09 ;-22,5 ; \mathrm{RJ}$

$-43,1 ;-22,5 ; \mathrm{RJ}$

$-38,97 ;-12,27$; Feira de Santana, BA

$-43,51 ;-20,29$; Ouro Preto, MG

-47,83; -15,86; Jardim Botânico de Brasília, Brasília, DF

-49,05; -16,74; Estação Ciência São José da Universidade Católica de Goiás, Goiânia, $\mathrm{GO}$

-47,85; -15,93; Jardim Botânico de Brasília, Brasília, DF

$-44,07 ;-19,93 ; \mathrm{MG}$

$-45,02 ;-23,53 ;$ Ubatuba, SP

$-54,61 ;-20,41$; fragmento de Cerrado, MS

-54,65; -20,44; fragmento urbano de Cerrado, Campo Grande, MT

-48,31; -18,92; Reserva Ecológica do Clube de Caça e Pesca Itororó, Uberlândia, MG

-48,03; -15,92; Mata de Açudinho, Fazenda Sucupíra; DF

-47,89; -15,94; Mata de Monjolo, Reserva Ecológica do IBGE; DF

$-48,47$; -27,6; parte central da Ilha de Santa Catarina, Florianópolis, SC

-44,07; -19,55; Reserva da Fazenda Jaguara, Matozinhos, MG

-47,93; -15,78; Jardim Botânico de Brasília, Brasília, DF

$-51,7 ;-17,9 ;$ Jataí, GO

-51,12; -16,86; Estação Ciência São José, GO

-49,24; -16,82; Estação Ciência São José, GO

-47,88; -15,95; Reserva Ecológica do IBGE, DF

$-50,14 ;-15,93$; campus II da Universidade Católica de Goiás, GO

-50,13; -15,95; campus II da Universidade Católica de Goiás, GO

$-43,73 ;-19,33$; Santana do Riacho, MG

-44,9; -18,76; Felixlândia, MG

$-46,52 ;-18,58 ;$ Patos de Minas, MG

-47,94; -15,94; Trecho de mata ciliar no córrego Capetinga, na Fazenda Água Limpa;

Brasília, DF

-47,93; -15,74; mata ciliar em cerrado do Planato Central Brasileiro, na Fazenda Água

Limpa; Brasília, DF

-47,93; -15,74; mata ciliar no córrego Capetinga, na Fazenda Água Limpa; Brasília, DF

-49,25; -16,68; Viveiro de Mudas da Prefeitura, Goiânia, GO

-45,-21,25; Fragmentos Florestais, Lavras, MG

$-45,1 ;-22,42 ; \mathrm{RJ}$ 
-43,23; -22,58; Parque Municipal Natural da Taquara, Duque de Caxias, RJ

-47,83; -15,83; Reserva Ecológica do Roncador, DF

-48,-16, Reserva Ecológica do Roncador, DF

$-43,9 ;-19,93$; Parque das Mangabeiras, Belo Horizonte, MG

$-54,92 ;-20,73$; Terenos, Terenos, MS

-54,59; -20,39; Campo Grande, Campo Grande, MS

-54,59; -20,39; Campo Grande, Campo Grande, MS

-54,59; -20,39; Campo Grande, Campo Grande, MS

-54,59; -20,39; Campo Grande, Campo Grande, MS

-54,59; -20,39; Campo Grande, Campo Grande, MS

-54,59; -20,39; Campo Grande, Campo Grande, MS

-56,15; -21,46; Margem esquerda do Rio da Prata, Jardins, MS

-47,84; -20,52; PCH Anhanguera, São Joaquim da Barra, SP

-47,8; -21,58; Estação Ecológia de Jataí, Luís Antônio, São Paulo

-40,56; -19,86; Augusto Ruschi (ex- Nova Lombardia), Santa Teresa, ES

$-40,8 ;-21,02$; Barra do Itapemirim, Itapemirim, ES

-45,07; -23,57; Ilha Anchieta, Ubatuba, São Paulo

-46,7; -21,72; São Sebastião da Grama, São Paulo

-46,73; -21,6; Divinolândia, São Paulo

-47,48; -20,26; Pedregulho, São Paulo

$-46,61 ;-20,72$; Passos, Minas Gerais

$-43,89 ;-19,63$; Lagoa Santa, Minas Gerais

-42,52; -14,92; Morro do Chapéu, Ignorada, Bahia

-47,33; -22,73; Parque Municipal de Americana, Americana, São Paulo

-47,96; -15,97; Fazenda Água Limpa - UNB, Brasília, Distrito Federal

-47,06; -22,91; Bosque de Campinas, Campinas, São Paulo

$-42,9 ;-20,25$; Rio Doce, Ignorada, Minas Gerais

-46,51; -20,62; Passos, Distrito Sad Joao Do Gloria, Fazenda Bela Vista, Mata Da Paca;

Minas Gerais

$-48,43 ;-18,48$; Araguari, Piracaiba; Minas Gerais

-39,09; -14,83; Ilheus, Fazenda Pirataquisse; Bahia

-44,33; -18,44; Curvelo, Thomaz Gonzaga, Fanzenda Boa Esperanca; Minas Gerais

-39,18; -14,66; Ilheus, Fazenda Alamada; Bahia

$-48,3$; -18,46; Uberlandia, $50 \mathrm{~km} \mathrm{~N}$; Minas Gerais State

-48,74; -13,77; Formoso, 24 km SE; Goias State

-47,88; -15,94; Reserva Ecológica do Roncador- IBGE, Distrito Federal

-48,03; -15,92; Fazenda Sucupira- Embrapa, Distrito Federal

-47,89; -15,95; Reserva Ecológica do Roncador- IBGE, Distrito Federal

-44,92; -21,3; Serra do Carrapato, Lavras, Minas Gerais

$-52,61 ;-23,66$; Cianorte, Paraná

-49,27; -25,43; Curitiba, Parque Barigui; Paraná

-48,51; -25,52; Paranaguá, Floresta Estadual do Palmito; Paraná

-49,21; -25,54; São José dos Pinhais, Renaut; Paraná

-51,94; -23,43; Maringá, Parque Ingá; Paraná

-43,88; -19,63; Rio das Velhas, near Lagoa Santa; Minas Gerais

-44,63; -15,74; SAO JANUARIA, 25 MI S OF; RIO SAO FRANCISCO; MINAS

GERAIS

-39,-15,83; Ilheus, Fazenda Almada; Bahia

-39,03; -14,79; ILHEUS; FAZENDA PIRATAQUISSE, BAHIA

-40,3; -12,53; Ilheus, Fazenda Morro de Pedra; Bahia 
-43,28; -21,75; Fazenda Floresta , Juíz de Fora, MG

-43,9; -21,71; Mata Grande, Parque Estadual Ibitipoca; MG

-43,86; -21,71; Mata do Patuá, MG

$-43,88 ;-21,65$; Mata dos Luna, MG

-43,98; -20,08; Estação Ecológica Fechos, Belo Horizonte, MG

-48,23; -20,02; Reserva Volta Grande, Conceição das Alagoas, MG

-48,2; -9,23; Canabrava, Rio Tocantins;

$-47,7 ;-12,55$; Palma,

$-49,45 ;-14,68$; Pilar,

-49,03; -14,58; Rio das Almas,

-47,52; -14,12; Veadeiros, Rio Corumbá;

$-49,73 ;-15,45$; Rio Uruhu,

-49,33; -15,75; Jaraguá, Rio das Almas;

$-47,67 ;-15,62$; Planaltina,

-52,25; -15,92; Aragarças, Rio Araguaia;

-48,97; -16,33; Anápolis,

-49,5; -16,37; Inhumas,

-49,32; -16,38; Goiânia,

$-49,22 ;-18,42$; Itumbiara, Rio Paranaíba;

-47,95; -18,17; Catalão, Rio Jordão;

$-45,-12,13$; Barreires, Bahia

-44,73; -11,8; Riachão das Neves, Bahia

-40,35; -10,77; Lamarão, Bahia

$-38,52 ;-12,98$; Salvador, Bahia

$-41,12 ;-12,98$; Macaco Secco, Bahia

$-40,97 ;-15,27$; Mundo Novo, Bahia

-39,2; -14,63; Fazenda Almada, Bahia

-39,32; -14,95; Buerarema, Ribeirão da Fortuna; Bahia

$-39,03 ;-14,82$; Morro das Pedras, Ilheus; Bahia

$-39,05 ;-14,65$; Urucutuca, Bahia

-39,27; -14,8; Itabuna, Rio Ilheus; Bahia

$-38,95 ;-15,65$; Rio Pardo, Bahia

$-39,22 ;-17,35$; Rio Jucurucu, Bahia

$-48,1 ;-18,43$; Rio Jordão, MG

$-48,18 ;-18,63$; Araguari, MG

$-48,3 ;-19,7$; Verissimo, MG

$-47,63 ;-18,88$; Água Suja , MG

$-47,92 ;-19,75$; Uberaba, MG

-46,5; -20,63; São João do Glória, MG

-44,35; -15,5; Januária, rio São Francisco; MG

$-44,93 ;-17,35$; Pirapora, MG

$-44,57$; $-17,9$; Lassance, MG

-44,18; -17,9; Buenopolis, MG

$-44,42 ;-18,75$; Curvelo, MG

-45,18; -18,83; Barra do Paraopeba, MG

-43,97; -19,67; Lagoa Santa, Rio das Velhas; MG

$-44,63 ;-22,38$; Serra do Itatiaia, MG

$-51,07 ;-20,12$; Rio Grande, SP

$-48,55 ;-20,55$; Barretos, Rio Grande; SP

-47,8; -19,98; Porto do Rio Paraná, SP 
-43,68; -18,36; João Vasse, Diamantina, MG

$-43,74 ;-18,36$; Barão de Guaicuhy, Diamantina, MG

-43,69; -18,38; Fazenda do Geraldo, Diamantina, MG

\section{Mico melanurus}

-55,29; -14,82; Hidrelétrica do Aproveitamento Múltiplo de Manso, rio Manso; MT

$-59,13 ;-8,57 ;$ AM

$-58,62 ;-8,35 ; \mathrm{AM}$

$-61,68 ;-9,9 ;$ Aripuanã, MT

-55,58; -15,5; Rio Aricá, MT

-56,08; -15,87; Fazenda Maravilha, Santo Antônio do Leverger, MT

-54,96; -14,94; Palmeiras, MT

-57,68; -16,07; Cáceres, MT

$-55,75 ;-15,46$; Chapada dos Guimarães, MT

$-57,64 ;-19,15$; Urucum, MS

-57,6; -19,Corumbá, MS

-63,57; -12,05; BR-429,São Francisco do Guaporé; RO

$-47,29 ;-23,2 ;$ Jururu, Salto; MT

-55,57; -15,49; Rio Aricá, MT

$-58,46$; -15,85; Porto Esperidião, MT

-61,69; -9,9; Cidade Humboldt, Rio Aripuanã; MT

-57,65; -19,01; Corumbá, MS

$-55,43 ;-20,45$; Palmeiras, MS

-62,79; -11,7; Fazenda do Mansur, São Miguel, RO

-63,31; -12,03; Sítio do Geraldo, São Francisco, RO

-61,46; -13,46; Fazenda São Paulo, Pimenteiras, RO

-55,58; -15,5; Rio Arica, MT

-55,86; -15,34; Chapada dos Guimarães, MT

-57,64; -19,15; Rio Aripuanã, Cidade Humboldt, MT

$-63,39 ;-11,19 ; \mathrm{RO}$

$-62,78 ;-12,53 ; \mathrm{RO}$

$-61,65 ;-9,84 ; \mathrm{RO}$

$-63,06 ;-8,95 ; \mathrm{RO}$

$-57,44 ;-17,68 ;$ MT

$-55,86 ;-15,35 ; \mathrm{MT}$

$-57,48 ;-16,86 ;$ MT

$-57,21 ;-15,67$; MT

$-59,25 ;-12,08 ; \mathrm{MT}$

-57,66; -19,01; Corumbá, MS

-57,65; -19,07; Maciço de Urucum, Corumbá, MS

-57,7; -19,03; Fronteira Brasil-Bolívia, Corumbá, MS

-57,49; -18,04; Serra do Amolar, Corumbá, MS

-57,66; -19,01; Corumbá, MS

-57,66; -19,01; Corumbá, MS

-57,66; -19,01; Corumbá, MS

-57,65; -19,07; Maciço de Urucum, Corumbá, MS

-57,66; -19,07; Maciço de Urucum, Corumbá, MS

-57,7; -19,03; Fronteira Brasil-Bolívia, Corumbá, MS 
-57,7; -19,02; Fronteira Brasil-Bolívia, Corumbá, MS

-57,7; -19,03; Fronteira Brasil-Bolívia, Corumbá, MS

-57,7; -19,03; Fronteira Brasil-Bolívia, Corumbá, MS

-57,49; -18,04; Serra do Amolar, Corumbá, MS

-60,93; -14,55; Los Fierros, Noel Kempff Mercado National Park, Bolivia;

-59,77; -20,02; Agua Dulce, 3 Km E, Parque Nacionales-Defensores Del Chaco; Alto

Paraguay

-66,17; -17,33; Cercado, Cercado, Cochabamba

$-60,63 ;-9,17$; Aripuana, Mato Grosso

-63,03; -7,52; Humaita, Br. 230,Km 969; Amazonas

$-55,83 ;-14,87$; Reservatório do Rio Manso, Mato Grosso

-63,06; -17,79; Jardim Botânico de Santa Cruz, Santa Cruz - Bolívia

-63,15; -17,93; Parque Regional Lomas de Arena, Santa Cruz - Bolívia

$-59,13 ;-8,57$; Serra do Sucunduri, AM

$-58,62 ;-8,35$; Rio Bararati, AM

-62,62; -14,63; Perseverancia, Santa Cruz - Bolívia

-62,75; -15,58; Arroyo Chuchui, Santa Cruz - Bolívia

-62,1; -14,5; San Martin, Santa Cruz - Bolívia

-63,97; -14,72; Rio Negro Caimanes, Santa Cruz - Bolívia

-61,8; -15,05; Orquiriquia, Santa Cruz - Bolívia

-63,32; -15,05; El Tutumo, Santa Cruz - Bolívia

-63,17; -17,83; Santa Cruz de la Sierra, Santa Cruz - Bolívia

-62,58; -16,75; Palmarito, Rio San Julian; Santa Cruz - Bolívia

-62,5; -16,5; Rio Quiser, Santa Cruz - Bolívia

-63,25; -17,75; Cercado, Santa Cruz - Bolívia

-63,28; -17,87; Mapaíso, Rio Grande; Santa Cruz - Bolívia

-61,-17,83; San José de Chiquitos, Santa Cruz - Bolívia

-57,78; -18,58; Puerto Suarez, Rio Paraguay; Santa Cruz - Bolívia

-60,33; -7,42; Castanho, Rio Castanhas; AM

-62,87; -8,05; Rio Jiparaná, alto Rio Madeira; RO

-61,85; -10,9; Urupá, Rio Jiparaná; RO

$-59,95 ;-15$,Vila Bella da Santissima Trindade, MT

$-58,55 ;-15,5$; Rio Jaurú, MT

-57,72; -16,05; Caiçara, Rio Paraguay; MT

-57,68; -16,07; São Luís de Cáceres, MT

-57,65; -19,02; Corumbá, Rio Paraguay; MT

$-57,55 ;-19,22$; Urucum, Rio Paraguai; MT

-56,08; -15,58; Cuiabá, Rio cuiabá; MT

$-55,8 ;-15,5$; Cabral, Rio Arica-Assu; MT

-55,75; -15,43; Santa Ana de Chapada, MT

-56,08; -15,87; Santo Antônio do Laverger, MT

-55,82; -15,67; Fazenda Aricá, MT

-55,5; -16,05; Palmeiras, MT

\section{Sapajus cay}

$-53,27 ;-22,3$; Bataiporã, MT

$-54,5 ;-20,95$; Campo Grande, MT

-53,17; -22,74; Porto Rico, PR 
$-53,18 ;-22,79 ;$ Porto Rico, PR

-53,18; -22,74; Porto Rico, PR

-53,14; -22,77; Porto Rico, PR

-56,5; -16,73; RPPN Sesc Pantanal,

$-56,1 ;-21,45$; Fazenda São Marcos, Guia Lopes da Laguna, MS

$-56,63 ;-21,1$; Parque Nacional da Serra da Bodoquena, MS

-54,61; -20,41; Parque Estadual Matas do Segredo (PEMS), MS

$-54,62 ;-20,41$; Parque Estadual Matas do Segredo (PEMS), MS

-54,87; -20,73; Reserva Particular do Patrimânio Natural, Nova Querência, MS

-54,87; -20,73; Reserva Particular do Patrimânio Natural, Nova Querência, MS

$-56,63 ;-21,1$; Distrito de Morraria do Sul, Bodoquena, MS

$-56,15 ;-21,48$; Jardim, Jardim, MS

$-56,12 ;-19,24$; Cassilândia, Cassilândia, MS

-51,64; -18,89; Itajá, GO

$-60,43 ;-13,47$; Comodoro, MT

$-57,65 ;-19,03$; Corumbá, MT

-57,65; -19,07; Maciço do Urucum, Corumbá, MT

-57,7; -19,03; Fronteira Brasil-Bolívia, Corumbá, MT

-57,5; -18,04; Serra do Amolar, Corumbá, MT

-63,73; -22,11; Playa Ancha, General José de San Martín, Salta

-63,4; -27,72; Santiago del Estero

-50,33; -16,08; Goias, Jose Alves Dos Santos Ranch; Goias

-57,55; -25,53; Villeta, Nueva Italia; Central- Paraguai,

-56,43; -21,45; Cabeceira do Prata, reserva privada; MS

-50,33; -16,08; Goias, Jose Alves Dos Santos Ranch; Goias

-53,27; -22,7; Alto Rio Paraná, MS

-53,31; -22,73; Alto Rio Paraná, MS

-53,27; -22,74; Alto Rio Paraná, MS

-53,21; -22,72; Alto Rio Paraná, MS

-56,25; -16,75; PRNH SESC, MT

-65,77; -27,67; Embalse Escaba, Dique Escaba, Tucuman

$-64,53 ;-22,43$; Foz do rio Lipeo, Salta

-64,63; -24,7; Parque Nacional El Rey, Dep Anta, Salta

-64,48; -24,12; Sierra Santa Bárbara, Jujuy

$-64,97 ;-24,13$; Garrapatal, Jujuy

$-64,37 ;-24,28$; Sierra Centinela, Jujuy

$-64,03 ;-22,28$; Sierra de Itau,

$-63,98 ;-22,35$; Reserva Provincial Acambuco,

-65,28; -22,38; Sierra de Santa Victoria,

$-63,9 ;-22,42$; Sierra de Tartagal,

$-64,85 ;-22,48$; Parque Nacional Baritu,

$-64,62 ;-22,67$; Bacia do Rio Pescado,

$-64,52 ;-22,9 ;$ Bacia do Rio Iruya,

$-65,08 ;-23,05$; Sierra de Zenta,

-64,62; -23,08; Bacia do Rio San Andrés,

-64,23; -23,28; Bacia do Rio Santa Maria,

-64,78; -23,78; Sierra de Calilegua,

$-69,48 ;-28,4$; Bacia do Rio Bermejo,

$-63,88 ;-18,75 ; 14.5 \mathrm{~km}$ NW de Masicuri, Santa Cruz

$-63,5 ;-21,25$; Villa Montes, Tarija 
$-63,9 ;-21,7$; Sierra Santa Rosa, Tarija

$-63,77 ;-21,82$; Carapari, Tarija

$-63,75 ;-22,03$; Yacuiba, Tarija

$-64,55 ;-22,27 ; 3 \mathrm{~km}$ SE de Cuyambuyo, Tarija

$-64,43 ;-22,68$; Rio Lipeo, Tarija

$-55,75 ;-15,43$; Chapada, MS

$-56,83 ;-17,16$; Fazenda Jofre, Pantanal; MS

-54,8; -17,95; Rio Piqueri, Coxim, MS

$-57,65 ;-19,02$; Corumbá, MS

$-57,63 ;-19,15$; Urucum, MS

$-56,47 ;-20,18 ;$ Salobra, MS

$-56,37 ;-20,25$; Miranda, MS

-52,17; -21,25; Fazenda Santa Barbara, MS

$-53,7 ;-23,23$; Rio Ivanheima, MS

-57,13; -21,27; Leste do Rio Paraguai, Salobra, MS

$-55,17 ;-21,6$; Maracaju, MS

-57,73; -11,42; Rio Arraia, Manissauá-Missu, Alto Xingu; MT

$-52,42 ;-12,03$; Posto Jacaré (Kamaiurá), Rio Kuluene, Parque do Xingu; MT

$-53,38 ;-12,25$; Lagoa Ipavu, Parque do Xingu; MT

-52,85; -12,93; Rio Kuluene, afluente do Xingu; MT

-52,75; -13,28; Mata do 7 de Setembro, Porto Garapu, Rio 7 de Setembro; MT

-51,4; -13,5; São Domingos, Rio das Mortes; MT

$-52,35 ;-14,68$; Xavantina, MT

-56,08; -15,62; Capão do Boi, Cuiabá, MT

-55,88; -15,7; Fazenda Aricá, Rio Aricá; MT

$-52,25 ;-15,88$; Barra do Garças, MT

-57,85; -15,07; Porto Esperidião, Rio Jauru, Poção; Cáceres, MT

$-57,77 ;-16,37$; Jacutinga, Rio Jauru; MT

$-55,7 ;-24,52 ; 40 \mathrm{~km} \mathrm{~N}$ de Curuguaty, Canindeyu,

-58,28; -20,28; Parque Nacional Cerro Corá,

-55,08; -24,3; M’baracayu Reserve,

$-56,03 ;-25,43$; Parque Nacional Caaguazu,

$-56,23$; -25,5; Santa Bárbara,

$-56,47 ;-25,75$; Guiara, Villarrica;

$-57,05 ;-26,02$; Y'bicui National Park,

$-54,75 ;-26,05$; Bosque Protector Nacuday,

$-55,-26,1$; Bosque Protector Yakui,

$-57,73$; -26,5; Santa Catalina, Chaco Paraguaio;

$-58,63$; -27,3; Margem Esquerda do Rio Paraguai,

\section{Sapajus libidinosus}

-47,27; -16,19; Aproveitamento Hidrelétrico de Queimado, MG

-48,03; -15,92; Mata de Açudinho, Fazenda Sucupíra; DF

-47,89; -15,94; Mata de Monjolo, Reserva Ecológica do IBGE; DF

-49,25; -16,68; Viveiro de Mudas da Prefeitura, Goiânia, GO

$-46,73 ;-10,92$; Jalapão, TO

$-45,-9$,Fazenda Boa Vista, PI

$-47,32$; -20,5; Ribeirão dos Correias e seus afluentes, Franca, SP 
-47,3; -20,53; Ribeirão dos Correias e seus afluentes, Franca, SP

$-47,93 ;-15,78 ;$ DF

-45,34; -9,83; Fazenda Boa Vista, Gilbués, PI

$-49,12 ;-16,5$; Goiânia, GO

$-49,18 ;-16,57$; Goiânia, GO

$-48,08 ;-15,58 ; \mathrm{DF}$

$-47,88 ;-15,75 ;$ DF

$-42,61 ;-8,67 ;$ PI

$-37,9 ;-6,07$; Martins, RN

$-37,93 ;-6,08$; Portalegre, RN

$-38,37$; -6,38; Luiz Gomes, RN

-37,98; -6,18; Serrinha dos Pintos, RN

$-38,32 ;-6,4$; Major Sales, RN

$-38,27 ;-6,32 ;$ José da Penha, RN

$-38,17$; -6,28; Marcelino Vieira, RN

$-37,78 ;-6,27$; João Dias, RN

$-37,83 ;-6,15$; Frutuoso Gomes, RN

$-50,63 ;-10,9$; Vale da Serra - borda do Rio Zacarias, Nova Xavantina, MT

-48,43; -18,48; Araguari, Piracaiba, Fazenda Piracaiba; Minas Gerais

-39,32; -7,65; Serrita, Cariri-Mirm, Sitio Ferreira Vicente, Agua Do Pingo; Pernambuco

-47,92; -19,75; Uberaba, Capao Do Cachorro; Minas Gerais

$-48,43$; -18,48; Araguari, Piracaiba, Fazenda Do Grotao; Minas Gerais

$-48,7 ;-18,58$; Tupaciguara, Minas Gerais

-48,18; -18,63; Araguari, Minas Gerais

$-49,02 ;-17,3$; Pouso Alto, Fazenda Taquari; Goias

$-48,63 ;-17,75$; Caldas Novas, Fazenda Cachoeirinha, Mata Cachoeirinha; Goias

$-46,87 ;-17,22$; Paracatu, Minas Gerais

$-44,55 ;-14,17$; Carinhanha, Cocos, Mata Do Boi, Fazenda Da Tolda; Bahia

-45,2; -11,05; Itajui, Fazenda Aldeia; Bahia

-48,18; -8,98; Pedro Afonso, Fazenda Mirador, Mata Mirador; Goias

-47,47; -7,33; Carolina, Fazenda Madeira, Cabeceira Damazio; Maranhao

-39,32; -7,93; Serrita, Cariri-Mirim, Sitio Boi, Morro Redondo; Pernambuco

-39,78; -7,68; Exu, Timorante, Serra Da Luveja, Pedra Da Ventania; Pernambuco

$-36,63 ;-9,4$; Palmeira dos Índios, Alagoas

-39,72; -7,35; Fazenda Catareno, NE de Exu; Exu, Pernambuco

$-39,55 ;-7,65$; Serrita, Caririmirim; Pernambuco

$-53,33 ;-22,76$; Mato Grosso do Sul

$-53,3 ;-22,72 ;$ Mato Grosso do Sul

$-53,21 ;-22,72$; Mato Grosso do Sul

$-42,49 ;-8,78$; Parque Nacional da Serra da Capivara, Piaui

-47,93; -15,74; Parque Nacional de Brasília, DF

-45,38; -9,67; Fazenda Boa Vista, Piauí

-45,42; -9,65; Fazenda Boa Vista, Piauí

-48,03; -15,92; Fazenda Sucupira- Embrapa, Distrito Federal

-47,89; -15,95; Reserva Ecológica do Roncador- IBGE, Distrito Federal

-49,26; -16,61; Campus Samambaia - UFG, Goiânia, GO

\section{Sapajus nigritus}


-53,74; -23,35; Paredão das Araras, Porto Figueira, PR

$-53,67 ;-26,52$; Ouro Verde, Guaraciaba, SC

$-42,67 ;-20,55 ; \mathrm{MG}$

$-42,42 ;-20,72 ; \mathrm{MG}$

-42,17; -19,74; Fazenda Monte Claros, Caratinga, MG

-42,37; -20,57; Fazenda Neblina, MG

-42,53; -20,88; Fazenda Neblina, MG

-40,5; -19,77; Santa Tereza, ES

$-40,58 ;-19,92$; Santa Tereza, ES

$-40,48 ;-20,3$; Cariacica, ES

$-40,53 ;-20,32$; Cariacica, ES

-41,01; -20,42; Domingos Martins, ES

$-41,01 ;-20,67$; Vargem Alta, ES

$-40,53 ;-19,95 ;$ ES

$-40,6 ;-19,94 ;$ ES

$-42,28 ;-22,56 ; \mathrm{RJ}$

$-43,35 ;-21,76$; Juiz de Fora, MG

$-51,7 ;-28,5$; São Jorge, RS

$-47,96 ;-24,14 ;$ SP

-44,55; -22,42; Trilha Maromba-Lamego, RJ

-44,62; -22,43; Trilha Maromba-Lamego, RJ

-44,58; -22,42; Trilha dos Três Picos, RJ

-44,6; -22,43; Trilha dos Três Picos, RJ

$-42,73 ;-20,05 ; \mathrm{RJ}$

$-41,8 ;-20,43$; entorno do PARNA do Caparaó, MG

$-43,05 ;-22,55 ; \mathrm{RJ}$

$-42,72 ;-20,09$; Fazenda Guapimirim, RJ

$-51,02 ;-23,27$; Mata Doralice, Ibiporã, PR

-48,19; -22,68; Sarâ, Fazenda Barreiro Rico; SP

-48,85; -26,3; Serra Dona Francisca, Juinville, SC

-47,72; -23,81; Fazenda João XXIII, Pilar do Sul, SP

$-47,95 ;-24,14 ;$ SP

-48,1; -22,68; Viraeiro/Tabatinguera (Fazenda Barreiro Rico), SP

-45,47; -22,75; Fazenda São Sebastião do Ribeirão Grande - Serra da Mantiqueira,

Pidamonhangaba, SP

$-48,13$; -22,79; Fazenda Barreiro Rico, SP

$-42,73 ;-20,05$; Fazenda Esmeralda, MG

$-43,88 ;-21,71$; Lima Duarte, MG

-46,25; -21,38; Mata da Olaria na Fazenda Lagoa, Monte Belo, MG

-42,74; -20,07; Fazenda Esmeralda, Rio Casca, MG

-42,74; -20,12; Fazenda Esmeralda de Cima, Rio Casca, MG

-42,74; -20,06; Fazenda Esmeralda, Rio Casca, MG

-42,73; -20,02; Fazenda Córredo do Ouro, Rio Casca, MG

-42,73; -20,07; Fazenda Esmeralda, Rio Casca, MG

$-41,78 ;-19,62 ; \mathrm{MG}$

-42,14; -19,79; Fazenda Montes Claros, Caratinga, MG

-42,68; -20,14; Fazenda Esmeralda, MG

$-42,77 ;-20,08 ;$ MG

$-42,42 ;-20,66 ; \mathrm{MG}$

$-41,82 ;-19,73$; Caratinga, MG 
$-47,96 ;-24,15 ; \mathrm{RJ}$

-42,45; -22,35; Macaé de Cima, RJ

-42,58; -22,47; Macaé de Cima, RJ

$-41,89$; -21,89; Boqueirão da Mata na Serra Grande, RJ

$-44,75 ;-22,32 ; \mathrm{RJ}$

$-44,83 ;-22,75 ; \mathrm{RJ}$

$-43,1 ;-22,5 ; \mathrm{RJ}$

$-44,75 ;-23,15$; Pedra Branca, Paraty, RJ

$-47,47 ;-22,37$; Fazenda São José, SP

-53,17; -22,74; Porto Rico, PR

$-53,18 ;-22,79$; Porto Rico, PR

$-53,18 ;-22,74$; Porto Rico, PR

$-53,16 ;-22,77$; Porto Rico, PR

$-47,99 ;-24,12 ; \mathrm{SP}$

$-51,23 ;-30,03$; Fragmento de floresta, Porto Alegre, RS

$-46,76 ;-23,46 ;$ SP

$-47,95 ;-24,14 ; \mathrm{SP}$

$-47,93 ;-24,17 ; \mathrm{SP}$

$-51,05 ;-23,27$; Mata Doralice, Ibiporã, PR

$-42,56 ;-19,7 ; \mathrm{MG}$

$-42,55 ;-19,71 ; \mathrm{MG}$

$-51,24 ;-23,51 ; \mathrm{PR}$

$-51,95 ;-23,91 ; \mathrm{PR}$

$-40,55 ;-19,9 ;$ ES

-44,71; -23,37; Serra Paranapiacaba, SP

-49,85; -24,1; Barra Mansa Farm, Arapoti, PR

$-43,21 ;-22,9$; Maciço da Tijuca, RJ

$-48,43 ;-24,33 ; \mathrm{SP}$

$-46,47 ;-23,49 ; \mathrm{SP}$

$-46,52 ;-23,49 ; \mathrm{SP}$

$-47,32 ;-20,5$; Fragmentos da área de manancial do rio Canoas, Franca, SP

$-46,53 ;-23,46 ; \mathrm{SP}$

$-47,99 ;-24,15 ; \mathrm{SP}$

$-47,92 ;-24,16 ; \mathrm{SP}$

-46,76; -23,46; Parque Jaraguá, SP

$-50,74 ;-22,9$; Florínea, SP

$-46,44 ;-23,53 ;$ SP

$-46,77 ;-23,46 ;$ SP

$-46,52 ;-23,49 ; \mathrm{SP}$

$-46,51 ;-23,5 ; \mathrm{SP}$

-47,81; -21,18; Mata urbana, Ribeirão Preto, SP

$-48,4 ;-24,35 ; \mathrm{SP}$

-51,-23,25; Parque Municipal Arthur Thomas, Londrina, PR

$-51,25 ;-23,5$; Parque Municipal Arthur Thomas, Londrina, PR

$-48,43 ;-24,34 ;$ SP

$-42,64 ;-19,8 ;$ MG

$-42,47 ;-19,49 ; \mathrm{MG}$

$-45,97 ;-22,22$; Parque Municipal de Pouso Alegre, Pouso Alegre, MG

$-41,1 ;-20,52$; Castelo, ES

$-41,13 ;-20,52$; Castelo, ES 
-41,08; -20,52; Fazenda Forno Grande, ES

$-40,53 ;-19,95 ;$ Santa Teresa, ES

$-40,67 ;-19,63$; ES

$-40,75 ;-19,77$; ES

-40,1; -19,48; Fragmento florestal (M7/317), ES

$-40,11 ;-19,49$; Fragmento florestal (Puriti), ES

$-42,53 ;-19,53 ; \mathrm{MG}$

$-53,63 ;-27,42$; Terra Indígena da Guarita, RS

-53,92; -27,17; Parque Estadual do Turvo, Derrubadas, RS

$-48,55 ;-22,3$; Reserva ecologica amadeu botelho,

-53,46; -22,77; APA das Ilhas e Va?rzeas do Rio Paraná, PR

-47,-24,31; Juréia - Itatins, Peruíbe, São Paulo

-47,24; -23,96; Juquitiba, São Paulo

-41,02; -20,4; PE Pedra Azul, Domingos Martins, ES

-40,67; -19,82; Floresta da Capela São Braz, Santa Teresa, ES

-40,67; -19,83; Floresta da Capela São Braz, Santa Teresa, ES

-40,37; -19,83; Pau Gigante, atualmente Ibiraçu; Ibiraçu, ES

$-40,47 ;-19,53$; Colatina, ES

-40,53; -20,1; Córrego Jequitibá, Santa Leopoldina, ES

-40,53; -20,1; Rio Formoso, Santa Leopoldina, ES

-40,58; -19,92; Colônia do Milanez, Santa Teresa, ES

-41,29; -20,76; FN de Pacotuba, Cachoeiro de Itapemirim, ES

-40,54; -19,97; Estação Biológica de Santa Lúcia, Santa Teresa, ES

-40,74; -20,06; Fragmento Florestal, Santa Maria de Jetibá, ES

-40,61; -19,94; Estação Biológica São Lourenço, Santa Teresa, ES

-40,56; -19,86; REBIO Augusto Ruschi (Nova Lombardia), Santa Teresa, ES

-40,62; -19,53; Vila Colatina; Margem direita do Rio Doce, Colatina, ES

-40,49; -20,24; REBIO Duas Bocas, Cariacica, ES

-45,08; -23,28; Estrada Santa Virgínia/Cunha, Trilhas Itamambuca e Rio dos Veados;

São Luís do Paraitinga, São Paulo

-47,77; -22,41; seguindo pela estrada sentido Fazenda Paredão, após a sede da Fazenda

Santo Antônio do Rochedo, proprietário Sr. Alfredo Barthman; Ipeúna, São Paulo

-47,84; -22,31; Fazenda Sinimbú, arrendada pela RIPASA; Itirapina, São Paulo

-47,88; -22,36; seguindo pela estrada da cachoeira do Saltão, próximo ao início de

descida da cuesta; Itirapina, São Paulo

-47,78; -24,05; São Miguel Arcanjo, São Paulo

$-53,27 ;-22,77$; Porto Rico, Paraná

$-51,55$; -23,6; Apucarana, Jandaia, Mata Pradoximo; Parana

-45,93; -21,35; Alfenas, Fazenda Primavera, Capao Das Perobas; Minas Gerais

-51,19; -23,Sertanopolis, Primeiro De Maio, Sitio Santo Antonio, Mata Santo Antonio;

Parana

-53,78; -27,85; Tres Passos, Santo Augusto, Area Dos Indios, Do Estado; Rio Grande

Do Sul

-52,6; -27,1; Xapeco, Sao Domingos, Fazenda Esperanca; Santa Catarina

-52,32; -27,27; Concordia, Ita, Bank Of Rio Uruguai, Mata Da Corredeira Feia; Santa

Catarina

$-54,1 ;-26,62$; San Pedro, San Pedro, Misiones

-49,67; -26,92; Ibirama, Jose Boiteux, Alto Dollmann, Serra Do Fachinal, Companhia

Hanseatica; Santa Catarina

-51,65; -23,6; Apucarana, Jandaia, Mata Campanar; Parana 
$-51,48 ;-23,55$; Apucarana, Parana

-45,07; -23,43; Ubatuba, Serra Do Mar; Sao Paulo

-51,02; -22,8; Sertanopolis, Primeiro De Maio, Sitio Corrego Dos Limoeiros, Mata

Corrego Dos Limoeiros; Parana

-44,3; -23,Angra Dos Reis, Mambucaba, Pau Preto; Rio De Janeiro

-43,12; -22,15; Tres Rios, Bemposta; Rio De Janeiro

-51,83; -21,87; Presidente Venceslau, Fazenda Bandeirantes, Mata Da Conserva, Near

Lagoa Sao Paulo; Sao Paulo

-47,02; -21,47; Mococa, Fazenda Barra Alegre, Mata Da Barra; Sao Paulo

-45,95; -21,43; Alfenas, Campestre, Fazenda Da Pedra, Mata Da Pedra; Minas Gerais

-43,42; -21,95; Juiz De Fora, Ibitiguai, Fazenda Santa Mayalda; Minas Gerais

-43,33; -21,88; Mathias Barbosa, Fazenda Cabui, Mata Do Grotao; Minas Gerais

-42,68; -21,87; Alem Paraiba, Fazenda Paraiso; Minas Gerais

-46,38; -20,87; Alpinopolis, Fazenda Cachoeira, Mata Barro Branco; Minas Gerais

-40,6; -19,92; Santa Tereza, Colonia Dos Irmaos, Rasseli; Espirito Santo

$-53,28 ;-22,78$; Porto Rico, Paraná

$-53,37 ;-22,81$; Porto Rico, Paraná

$-53,31 ;-22,78$; Porto Rico, Paraná

-47,47; -22,37; Fazenda São José, São Paulo

-42,4; -20,59; Parque Estadual da Serra do Brigadeiro, Minas Gerais

-49,34; -25,99; Agudos do Sul, Paraná

$-48,71 ;-25,43$; Antonina, Paraná

-51,46; -23,55; Apucarana, Paraná

-49,41; -25,59; Araucária, Paraná

$-50,01 ;-24,79$; Castro, Paraná

$-52,47 ;-26,4$; Clevelândia, Paraná

-52,86; -22,66; Diamante do norte, EE Caiuá; Paraná

-51,98; -23,92; Fênix, PE Vila Rica do Espirito Santo; Paraná

$-54,59 ;-25,55$; Foz do Iguaçu, Paraná

-51,32; -26,43; General Carneiro, Paraná

$-48,33 ;-25,31 ;$ Guaraqueçaba, Paraná

$-50,19 ;-23,85$; Ibaiti, Paraná

$-51,05 ;-23,27$; Ibiporã, Paraná

$-52,2 ;-22,75$; Inaja, Paraná

-49,97; -23,16; Jacarezinho, Paraná

$-51,64 ;-23,6$; Jandaiá do Sul, Paraná

$-52,42 ;-25,41$; Laranjeiras do Sul, Paraná

-53,14; -22,92; Loanda, RPPN Faz. Matão; Paraná

-51,16; -23,31; Londrina, PE Mata dos Godoy; Paraná

-51,94; -23,43; Maringá, parque Ingá; Paraná

-48,54; -25,82; Matinhos, PM Rio da Onça; Paraná

-54,09; -25,3; Medianeira, Paraná

-48,88; -25,44; Morretes, PE Pico Marumbi; Paraná

-54,26; -24,08; PARNA 7 quedas, Paraná

-51,99; -26,48; Palmas, Paraná

-52,47; -23,07; Paranavaí, Paraná

$-52,67$; -26,23; Pato Branco, Paraná

$-49,06 ;-25,44$; Piraquara, Paraná

$-53,27 ;-22,77$; Porto Rico, Paraná

-53,18; -22,72; Porto São José, Paraná 
-49,08; -25,37; Quatro Barras, Paraná

-53,48; -23,08; Querência do Norte, Porto Natal; Paraná

-49,63; -23,6; Salto do Itararé, Paraná

-51,04; -23,06; Sertanópolis, Paraná

-50,62; -24,32; Telêmaco Borba, Paraná

-50,41; -24,51; Tibagi, Guartelá; Paraná

$-53,33 ;-23,77$; Umuarama, Paraná

$-48,63 ;-25,56$; Paranaguá, Alexandra; Paraná

$-51,21$; -23,33; Floresta da UEL, Londrina, Paraná

-53,14; -23,34; Parque A. Thomaz, Londrina, Paraná

-50,38; -24,13; Telemaco-Borba, Paraná

$-53,31 ;-22,78$; Mata de galeria do rio paraná, Telemaco-Borba, Paraná

-53,3; -22,85; Floresta Zezão, Porto-Rico, Paraná

$-53,33 ;-22,87$; Floresta G. Nogueira, Porto-Rico, Paraná

$-44,58 ;-22,42$; Parque Nacional do Itatiaia, RJ

$-48,15 ;-22,67$; Barreiro Rico, SP

-43,28; -21,75; Fazenda Floresta , Juíz de Fora, MG

-46,93; -22,84; Mata Ribeirão Cachoeira, Campinas, SP

$-43,9 ;-21,71$; Mata Grande, Parque Estadual Ibitipoca; MG

$-44,59 ;-22,42$; Parque Nacional do Itatiaia, RJ

$-43,77 ;-18,43$; Gouveia, MG

$-54,42 ;-25,72$; Parque Nacional Iguazú, Misiones

$-54,57 ;-25,6$; Parque Nacional Iguazú, Misiones

-40,63; -19,72; Pau Gigante, atualmente Ibiraçu; ES

-40,07; -40,43; Reserva Florestal Goitacazes, margem direita do Rio Doce; ES

-40,08; -19,47; Bebedouro, ES

-40,-19,47; 10km Eda BR-101,na estrada 440 para Regência; ES

-40,92; -19,5; Mascarenhas, ES

$-40,62 ;-19,53$; Colatina, ES

-40,82; -19,53; Itapina, ES

$-40,63 ;-19,57$; Baunilha, ES

-40,2; -19,58; Jacupemba, ES

$-40,62 ;-19,63$; Boapaba, ES

-39,83; -19,65; Reserva Biológica Comboios, ES

-40,85; -19,65; Itaimbé, ES

-40,28; -19,7; Corrego da Água, ES

-40,03; -19,73; Riacho, ES

$-40,67 ;-19,73$; São Roque, ES

-40,38; -19,75; João Neiva, ES

-40,5; -19,77; Reserva Biológica Nova Lombardia, ES

-40,28; -19,82; Aracruz, ES

-40,67; -19,82; Colônia dos Irmãos Rosseli, ES

-40,73; -19,87; Itarama, ES

-40,58; -19,92; Colonia do Milanez, ES

-40,13; -19,93; Coqueiral, ES

$-40,4 ;-19,93$; Fundão, ES

-40,73; -19,95; Alto Caldeirão, ES

-40,15; -19,97; Santa Cruz, ES

-40,63; -20,Lagoa do Rio Bonito, ES

-40,83; -20,Itapemirim, ES 
-40,75; -20,02; Santa Maria (Jetibá), ES

-40,53; -20,1; Córrego Jequitibá, Santa Leopoldina; ES

-40,28; -20,15; Reserva Florestal Mestre Álvaro, ES

$-41,5 ;-20,23$; Ibatiba, ES

$-41,07 ;-20,27$; Barcelos, ES

-40,48; -20,3; Reserva Florestal Duas Bocas, Cariacica; ES

$-40,67 ;-20,32$; Todos os Santos, ES

$-40,8 ;-20,32$; Paraju, ES

-41,2; -20,33; São João de Viçosa, ES

$-40,65 ;-20,37$; Domingos Martins, ES

$-40,5 ;-20,38 ;$ Viana, ES

$-40,62 ;-20,38$; Santa Isabel, ES

$-40,67 ;-20,4$; Marechal Floriano, ES

$-40,33 ;-20,47$; Nova Manta, ES

$-41,1 ;-20,5$; Parque Estadual Forno Grande, ES

-40,7; -20,57; Sagrada Família, ES

-41,08; -20,57; Patrimônio do Ouro, ES

$-41,2 ;-20,6$; Castelo, ES

-41,18; -20,62; Área de Floresta Permanente e Preservação da Flora, ES

-41,28; -20,73; Reserva Florestal Bananal do Norte, ES

-41,47; -20,77; Engenheiro Reeve, ES

-41,4; -20,78; Jerônimo Monteiro, ES

$-40,78 ;-20,9$; Itaoca, ES

-41,2; -20,9; Atílio Vivacqua, ES

-41,-20,95; Reserva Florestal Usina, ES

$-41,35 ;-20,95$; Muqui, ES

$-41,25 ;-19,33$; Resplendor, MG

$-42,3 ;-19,58$; Vargem Alegre, MG

-41,47; -19,68; Laginha do Mutum, MG

-42,13; -19,75; Fazenda Montes Claros, MG

-43,03; -19,77; Fazenda Esperança, São José da Lagoa, Rio Piracicaba; MG

-42,07; -19,78; Caratinga, MG

-42,55; -19,88; Rio Matipoo, MG

-41,67; -20,13; Areado, 3 km E de Laginha; MG

$-41,87 ;-20,25$; Martin Soares, MG

-41,73; -20,32; Parque Nacional de Caparaó, MG

$-46,85 ;-2,33$; Delfinópolis, MG

-41,93; -20,37; Manhumirim, MG

-43,85; -20,43; Fazenda Cágado, Pires Olegário; MG

-46,93; -20,6; Santa Rita de Cássia, MG

$-46,62 ;-20,72$; Passos, MG

$-42,85 ;-20,75$; Mata do Paraíso, MG

$-42,03 ;-20,9$; Tombos, MG

$-42,07 ;-21,55$; Serrania, MG

$-45,77 ;-21,6$; Concórdia, MG

$-43,88 ;-21,7$; Parque Estadual de Ibitipoca, MG

-43,4; -21,78; Fazenda Cabul, Mathias Barbosa; MG

-42,6; -21,88; Além Paraíba, MG

-51,03; -23,03; Mata Corrego do Limoeiro, PR

$-53,47 ;$-23,08; Porto Natal, ; Querência do Norte, PR 
-49,98; -23,15; Jacarezinho, PR

-53,72; -23,37; Porto Camargo, Rio Paraná; PR

-51,45; -23,57; Apucarana, PR

$-48,82 ;-25,25$; Serra Graciosa, PR

-54,08; -25,28; São Vicente Grande, PR

-53,72; -25,38; Parque Nacional Foz do Iguaçu, PR

-52,35; -26,4; Chacara Aparício, Clevelândia; Pato Branco, PR

$-41,97 ;-21,03$; Natividade, RJ

-41,12; -21,3; Fazenda São Pedro, João Pessoa; RJ

$-41,72 ;-22,17 ; 6 \mathrm{~km} \mathrm{~N}$ do desvio para Macaé da BR-101,RJ

$-44,67 ;-22,42$; Parque Nacional de Itatiaia, RJ

-42,28; -22,58; Reserva Biológica Poço das Antas, RJ

-44,57; -23,08; Mambucaba, RJ

$-44,23 ;-23,15$; Ilha Grande, RJ

$-44,65 ;-23,17$; Parati, RJ

$-44,77 ;-23,22$; Pedra Branca, RJ

-53,93; -27,55; Gleba do Pinhal, Santo Augusto; Três Passos, RS

$-54,97 ;-28,4 ;$ São Luís Gonzaga, RS

$-48,88 ;-26,03$; Fazenda Rio Turvo, Garuva, SC

-48,68; -26,25; Figueira, São Franciso do Sul, SC

$-48,83 ;-26,3$; Joinville, SC

$-48,23 ;-26,42$; Corupá, SC

$-49,27 ;-26,42$; Colônia Hansa, SC

-49,55; -27,05; Alto Dollman, Serra do Fachinal, Ibirama, Xapecó; Xapecó, SC

-52,68; -27,22; Fazenda Esperança, São Domingos, Xapecó; Xapecó, SC

$-50,27 ;-20,17$; Fernandópolis, SP

$-47,78 ;-20,35$; Ituverava, SP

$-47,38 ;-20,55 ;$ Franca, SP

-48,57; -20,57; Rio Grande, Mato Grosso de Barretos; SP

$-51,5 ;-20,63$; Itapura, SP

$-47,8 ;-21,17$; Ribeirão Preto, SP

$-50,87 ;-21,22$; Valparaíso, SP

-50,63; -21,25; Fazenda Conceição, Guararapes; SP

-49,95; -21,47; São Jerônimo, Avanhandava; SP

-49,75; -21,67; Campestre, Lins, SP

-52,1; -21,77; Presidente Epitácio, SP

-51,85; -21,95; Presidente Venceslau, Fazenda Bandeirantes; SP

-47,87; -21,97; São Carlos (Campus da UFSC), SP

-47,65; -22,13; Analândia, SP

$-49,08 ;-22,33$; Bauru, SP

-52,63; -22,33; Porto Cabral, Rio Paraná; SP

-52,25; -22,38; Morro do Diabo, SP

-49,65; -22,4; Reserva Estadual Caetetus, Gália; SP

$-52,17 ;-22,52$; Morro do Diabo, SP

$-45,18 ;-22,6$; Piquete, SP

$-48,15 ;-22,65$; Fazenda Barreiro Rico, SP

-45,47; -22,75; Fazenda São Sebastião do Ribeirão Grande, Pindamonhangaba; SP

-48,42; -22,9; Victória (=Botucatu), SP

$-49,92 ;-23$, Ourinhos, SP

-48,9; -23,1; Fazenda Santa Madalena, Avaré, SP 
-45,87; -23,2; São Francisco Xavier, São José dos Campos, SP

$-46,75 ;-23,27$; Francisco Morato, SP

$-46,6 ;-23,37$; Cantareira, SP

$-46,28 ;-23,43$; Paranapiacaba (= Alto da Serra), SP

$-46,62 ;-23,53$; Ipiranga, SP

$-46,55 ;-23,55$; Belém, SP

$-45,83 ;-23,67$; Boracéia, Salesópolis; SP

$-45,9 ;-23,72$; Varjão do Guaratuba, SP

$-47,4 ;-23,72$; Piedade, SP

$-45,33 ;-23,78$; Ilha de São Sebastião, SP

$-45,32 ;-23,8 ;$ São Sebastião, SP

-47,98; -23,88; São Miguel Arcanjo, SP

-46,6; -23,98; Estação Engenheiro Ferraz, SP

-49,33; -24,12; Itararé, SP

$-48,75 ;-24,6$; Lageado, Iporanga; SP

-47,77; -24,73; Parque Estadual Carlos Botelho, SP

$-47,88 ;-25$, Cananéia, SP

-57,77; -25,2; Santa Ana al Ihguasu, Paraguai

\section{Sapajus xanthosternos}

-39,03; -14,82; Ilhéus, BA

$-38,92 ;-15,81$; Passui, Belmonte, BA

-38,92; -15,81; Vitória, Fazenda Vitória; Belmonte, BA

$-43,81 ;-14,32$; Malhada, Serra do Iuiu, Fazenda da Serra; Carinhanha, BA

-40,-16,Salto de Divisa, MG

$-40,54 ;-15,85$; Bandeira, MG

$-41,-16,43$; Jequitinhonha, MG

$-39,25 ;-15,12 ; \mathrm{BA}$

$-39,42 ;-15,25 ; \mathrm{BA}$

$-39,08 ;-15,29$; Una, BA

$-38,95 ;-15,68$; Fazenda Santa Clara, Canavieiras, BA

-40,07; -15,12; Fazenda Quaiaquil, Itororó, BA

-40,14; -14,79; Fazenda Limoeiro, Nova Canaã, BA

-39,46; -14,32; Piancó, Gongoji, BA

$-40,25 ;-15,25$; Fazenda Camponesa, Itapetinga, BA

$-43,79 ;-14,3$; Serra Geral, Carinhanha, BA

$-39,7 ;-14,75$; Ilhéus, BA

$-38,93 ;-15,81$; Passui, Belmonte, BA

$-39,22 ;-17,35$; Rio Jucurucu, BA

$-43,79 ;-14,4$; Serra do Iuiu, BA

$-43,78 ;-14,36$; Malhada, BA

-39,83; -14,47; Rio Gongogi, BA

$-40,2 ;-10,45$; Senhor do Bonfim, BA

-39,07; -12,58; Governador Mangabeira, BA

$-39,72 ;-13,5$; Três Braços, BA

$-39,02 ;-13,3$; Valença, BA

$-39,13 ;-15,2$; BA

-39,08; -15,25; Estação Experimental Lemos Maia, Una, BA 
-39,1; -15,28; Estação Experimental Lemos Maia, Una, BA

-39,2; -15,38; Estação Experimental Canavieiras, BA

-39,22; -15,45; Estação Experimental Canavieiras, BA

$-39,08 ;-15,72$; Ouricana, BA

$-37,62 ;-11,23$; Arauá, SE

$-37,42 ;-11,25 ;$ Umbaúba, SE

$-37,75 ;-11,5$; Cachoeira do Abadia, SE

$-37,68 ;-11,53$; Jandaira, BA

$-38,2 ;-12,2 ;$ Araçás, BA

$-38,2 ;-12,37$; Catu, BA

-39,42; -12,95; São Miguel das Matas, BA

-39,25; -13,Santo Antônio de Jesus, BA

-39,-12,98; Nazaré, BA

$-39,53 ;-13,23$; Mutuípe, BA

$-39,6 ;-13,25$; Jiquiriçá, BA

$-39,7 ;-13,27$; Ubaíra, BA

$-38,98 ;-13,28$; Guaibim, BA

-39,08; -13,45; Taperoá, BA

$-39,12 ;-13,6$; Nilo Peçanha, BA

-39,5; -13,58; Teolândia, BA

-39,38; -13,77; Piraí do Norte, BA

-39,17; -13,75; Ituberá, BA

-39,12; -13,93; Camamu, BA

-39,38; -14,05; Itamarati, BA

$-39,5 ;-14,17$; Travessão, BA

$-39,38 ;-14,17$; Ibirapitanga, BA

$-39,75 ;-14,13$; Ipiaú, BA

$-39,33 ;-14,33$; Aurelino Leal, BA

-39,87; -14,27; Itagibá, BA

-39,92; -14,42; Dário Meira, BA

-39,88; -14,5; Ibitupã, BA

$-39,28 ;-14,6$; Uruçuca, BA

$-39,65 ;-14,7$; Almadina, BA

$-39,6 ;-14,87$; Ibicarai, BA

$-39,67 ;-14,8$; Barro Preto, BA

$-39,2 ;-14,78$; Salobrinho, BA

-39,08; -14,78; Ponto do Pitu, BA

$-39,32 ;-14,83$; Itabuna, BA

$-39,03 ;-14,85 ; 7 \mathrm{~km}$ ao sul de Ilhéus na estrada BA-001 para Olivença, BA

$-39,-15,03$; Olivença, BA

-39,93; -14,98; Firmino Alves, BA

$-40,07 ;-15,95$; Itororó, BA

$-39,03 ;-15,33 ; 4 \mathrm{~km}$ ao sul de Uma na estrada BA-001 de Canavieiras, BA

$-39,2 ;-15,37$; Vila São João, BA

$-39,95 ;-15,6$; Potiraguá, BA

$-40,07 ;-15,65$; Itarantim, BA

$-40,27 ;-15,62$; Maiquinique, BA

-39,4; -15,83; Santa Maria Eterna, BA

$-40,57 ;-15,88$; Bandeira, MG 
$-40,53 ;-16,08$; Terminal de travessia do Rio Jequetinhonha, na margem norte, a leste de Almenara; MG

$-40,7 ;-16,17$; Almenara, MG

$-39,25 ;-13,83$; Ituberá, BA

$-38,92 ;-15,81$; Passuí, Belmonte, BA

$-40,32 ;-10,51$; Senhor do Bonfim, BA

-40,91; -12,36; Reserva Indígena Pataxó, BA

-39,19; -14,66; Proximidades de Ilhéus, BA

-38,99; -15,46; Proximidades de Poxim do Sul, BA

$-39,12 ;-15,76$; Próximo de Ouricana, BA

-39,53; -15,92; Proximidades de Itapebi, BA

-37,22; -11,16; Área de Proteção Ambiental do Litoral Sul, SE

-37,31; -11,Fazenda Trapsa (Itaporanga d`Ajuda), SE

$-39,08 ;-15,29$; Belmonte, Vitoria, Fazenda Vitoria; Bahia

$-38,9 ;-15,85$; Belmonte, Passui; Bahia

-43,78; -14,3; Carinhanha, Malhada, Serra Do Iuiu, Fazenda Da Serra; Bahia

$-39,03 ;-14,82$; Ilheus, Bahia

$-37,23 ;-11,2$; Fazenda Trapsa, Sergipe

-39,08; -14,32; RPPN Reserva Capitão, Bahia

-41,01; -16,34; Mata Escura, Jataí, Duas Barras; Jequitinhonha, MG

-40,24; -15,36; Fazenda São Pedro, estrada entre Itapetinga e Maiquinique, próximo à balsa do rio Pardo; BA 


\section{ANEXO 5 - LISTA DAS INSTITUIÇÕES E REFERÊNCIAS QUE POSSUEM OS REGISTROS DE PRIMATAS DO CERRADO PRESENTES NO ANEXO 4.}

\section{Instituições}

American Museum of Natural History

Administración de Parques Nacionales, Argentina

Arctos- Museum of Vertebrate Zoology- mammal catalog(Alves 2005)

Avian Knowledge Network, Macaulay Library - Audio Data

Coleção de Mamíferos da Universidade Federal do Espírito Santo

Coleção de Mamíferos da UNEMAT - Campus Nova Xavantina

Coleção de Mamíferos do Museu de Zoologia Adão José Cardoso - UNICAMP

Coleção Mastozoológica DZUP- Universidade Federal do Paraná

FAPESP, Sinbiota

Field Museum of Natural History (Zoology) Mammal Collection

Fonoteca Neotropical Jacques Viellaird - UNICAMP

Fundación Miguel Lillo - Colección Mamíferos

GBIF-Sweden, Mammals (NRM)

MCP-Mamiferos - Coleção de Mamíferos da PUCRS

Museo Argentino de Ciencias Naturales

Museu de Biologia Professor Mello Leitão, Coleção de Mamíferos

Museu Nacional do Rio de Janeiro

Museum of Natural Science- Louisiana State University

Natural History Museum of Los Angeles County

National Museum of Natural History, Smithsonian Institution, Vertebrate Zoology Mammals Collections

Peabody Museum - Yale University

Zoological Museum - Natural History Museum of Denmark

\section{REFERÊNCIAS BIBLIOGRÁFICAS}

Abbehusen, A., R. M. L. Silva, and C. E. Barreto. 2005. Ecologia alimentar e área do uso do sagui-da-cara-branca, Callithrix geoffroyi (Humboldt, 1812), na Estação Vera-Cruz, Porto Seguro-BA.in XI Congresso Brasileiro de Primatologia.

Abbehusen, A., R. M. L. Silva, and C. E. Barreto. 2007. Dieta e área de uso do saguida-cara-branca (Callithrix geoffroyi) em Porto Seguro, Bahia.in J. C. BiccaMarques, editor. A PRIMATOLOGIA NO BRASIL.

Abreu, G. R., F. R. M. Silva, and M. A. O. Monteiro-da-Cruz. 1997. Atividade social durante o período de descanso diurno em grupos silvestres de Callithrix jacchus.in VIII Congresso Brasileiro de Primatologia.

Aguiar, L. M., G. Ludwig, and F. C. Passos. 2009. Group size and composition of black-and-gold howler monkeys (Alouatta caraya) on the Upper Parana River, Southern Brazil. Primates 50:74-77.

Aguiar, L. M., G. Ludwig, W. K. Svoboda, C. L. S. Hilst, I. T. Navarro, and F. C. Passos. 2007a. Occurrence, local extinction and conservation of Primates in the corridor of the Upper Paraná River, with notes on other mammals Revista Brasileira de Zoologia 24:898-906. 
AGUIAR, L. M., G. LUDWIG, W. K. SVOBODA, G. M. TEIXEIRA, C. L. S. HILST, M. M. SHIOZAWA, L. S. MALANSKI, A. M. MELLO, I. T. NAVARRO, F. C. M. M. PASSOS, L. S. MALANSKI, A. M. MELLO, I. T. NAVARRO, and PASSOS. 2007b. Use of Traps to Capture Black and Gold Howlers (Alouatta caraya) on the Islands of the Upper Paraná River, Southern Brazil. American Journal of Primatology.

Aguiar, L. M., D. M. Mellek, K. C. Abreu, T. G. Boscarato, I. P. Bernardi, J. M. D. Miranda, and F. C. Passos. 2007c. Simpatria entre Alouatta caraya e Alouatta clamitans e a re-descoberta de híbridos potenciais em vida livre no sul do brasil.in XII Congresso Brasileiro de Primatologia.

Aguiar, L. M. and F. C. Passos. 2007. Grupos mistos entre Alouatta caraya, Alouatta guariba clamitans e morfotipos híbridos em vida livre no sul do Brasil.in XII CONGRESSO BRASILEIRO DE PRIMATOLOGIA. SBPr, Belo HorizonteBrazil.

Aguiar, L. M., N. R. Reis, G. Ludwig, and V. J. Rocha. 2003. Dieta, área de vida, vocalizações e estimativas populacionais de Alouatta guariba em um remanescente florestal no norte do estado do Paraná. Neotropical Primates 11:78-86.

Aguiar, L. M., N. R. Reis, V. J. Rocha, and G. Ludwig. 2002. Área de uso de Alouatta guariba (HUMBOLDT, 1812) no remanescente florestal Mata Doralice, IbiporãParaná. Page 98 in X Congresso Brasileiro de Primatologia.

Albuquerque, A. C. S. R., M. C. L. Nascimento, H. M. Santos, and M. B. C. Sousa. 2004. Função ovariana e adrenocortical de fêmeas adultas do sagui (Callithrix jacchus) em relação ao comportamento de emigração.in S. L. Mendes and A. G. Chiarello, editors. A PRIMATOLOGIA NO BRASIL. SBPr.

Albuquerque, A. C. S. R., M. C. L. Nascimento, M. B. C. Sousa, and H. M. Santos. 1999. Atividade ovariana e comportamento de emigração de fêmeas adultas do sagui (Callithrix jacchus) vivendo no mesmo grupo no ambiente natural.in IX CONGRESSO BRASILEIRO DE PRIMATOLOGIA.

Albuquerque, A. C. S. R., M. B. C. Sousa, H. M. Santos, and T. E. Ziegler. 2001. Behavioral and Hormonal Analysis of Social Relationships Between Oldest Females in a Wild Monogamous Group of Common Marmosets (Callithrix jacchus). International Journal of Primatology 22:631-645.

Albuquerque, F. S. and M. D. F. Arruda. 1997. Socialização de filhotes de Callithrix jacchus em ambiente natural.in M. B. C. Sousa and A. A. L. Menezes, editors. A PRIMATOLOGIA NO BRASIL. SBPr, Natal- RN- Brazil.

Albuquerque, F. S., E. Otta, and M. F. Arruda. 2000. Comparação do cuidado de uma prole de gêmeos com uma prole de filhote único de Callithrix jacchus no ambiente natural. Pages 11-21 in C. Alonso and A. Langguth, editors. A Primatologia no Brasil. Sociedade Brasileira de Primatologia, João Pessoa- PB.

Alencar Araripe, A. C. and M. A. O. Monteiro da Cruz. 1997. Flexibilidade da organização social em uma amostra populacional do Callithrix jacchus.in VIII Congresso Brasileiro de Primatologia.

Almeida-Silva, B., A. A. Cunha, J. P. Boubli, S. L. Mendes, and K. B. Strier. 2005a. Population density and vertical stratification of four primate species at the Estação Biológica de Caratinga/RPPN-FMA, Minas Gerais, Brazil. NEOTROPICAL PRIMATES 13:25-29.

Almeida-Silva, B., P. G. Guedes, J. P. Boubli, and K. B. Strier. 2005b. Deslocamento terrestre e o comportamento de beber em um grupo de barbados (Alouatta 
guariba clamitans CABRERA,1940) em Minas Gerais. Neotropical Primates 13:1-3.

Almeida, L. M. I., O. D. S. V. Melo, and V. Boere. 2002. Observações sobre o comportamento alimentar do Sagui (Callithrix penicillata) em uma árvore de goma de Mata mesofítica de interlúvio, no Jardim Botânico de Brasília, DF.in X Congresso Brasileiro de Primatologia.

Alonso, A. C., J. N. H. Cabral, L. X. Lokschin, G. Marsicano, A. Roll, G. Buss, R. C. Printes, M. F. M. Santos, M. M. A. Jardim, and H. P. Romanowski. 2005. Programa Macacos Urbanos: manejo de bugio-ruivo (Alouatta guariba clamitans, CABRERA 1940) em Porto Alegre.in XI Congresso Brasileiro de Primatologia. SBPr, Porto Alegre- Brazil.

Alonso, A. C., I. Pfeifer, and L. Jerusalinsky. 2002. Ocorrência e distribuição do bugiopreto (Alouatta guariba clamitans) no Morro do Osso: Implpicações para a conservação. Page 80 in X Congresso Brasileiro de Primatologia.

Alonso, C. 1984. Observações de campo sobre o cuidado à prole e o desenvolvimento dos filhotes de Callithrix jacchus jacchus.in M. T. Melo, editor. A PRIMATOLOGIA NO BRASIL. SBPr.

Alonso, C., D. S. Faria, and D. P. Santee. 1986. Distribuição espacial e variação morfológica das populações de Callithrix jacchus na área do recôncavo bahiano.in M. T. Melo, editor. A PRIMATOLOGIA NO BRASIL. SBPr, Belo Horizonte.

Alonso, C. and A. Langguth. 1989. Ecologia e comportamento de Callithrix jacchus (Primates: Callithrichidae) numa ilha de Floresta Atlântica. Revista Nordestina de Biologia 6:105-138.

Alves, F. C. and V. B. Fortes. 2005. Germinação de sementes de banana-do-mato, Philodendron selloun (Araliaceae), consumidas pelo bugio-preto, Alouatta caraya (Primates: Atelidae). XI Congresso Brasileiro de Primatologia, Porto Alegre- Brazil.

Alves, S. L. 2005. Records of Primates at Itatiaia National Park, Brazil. Neotropical Primates 13:36-37.

Alves, S. L. and A. S. Zau. 2005a. Comportamento alimentar de Alouatta guariba clamitans na árrea de relevante interesse ecológico floresta da Cicuta, RJ.in XI Congresso Brasileiro de Primatologia, Porto Alegre- Brazil.

Alves, S. L. and A. S. Zau. 2005b. Estimativas populacionais de Alouatta guariba clamitans Cabrera, 1940 na área de relevante interesse ecológico Floresta da Cicuta, RJ.in XI Congresso Brasileiro de Primatologia. SBPr, Porto AlegreBrazil.

Alves, S. L. and A. S. Zau. 2005c. A importância da área de relevante interesse ecológico Floresta da Cicuta (RJ) na conservação do bugio-ruivo (Alouatta guariba clamitans Cabrera, 1940). Revista Universidade Rural, série Ciências da Vida 25:41-48.

Amaral, J. M. J. and D. De Jong. 1999. The behavior of the tufted capuchin monkey (Cebus apella) in an urban forest in Ribeirao Preto, SP.in IX Congresso Brasileiro de Primatologia.

Amoedo, P. and A. Abbehusen. 2005. Influência das alterações sazonais no comportamento alimentar e exploratório do Callithrix jacchus no Parque Metropolitano de Pituaçu, Salvador- Bahia.in XI Congresso Brasileiro de Primatologia, Porto Alegre- Brazil. 
Antunes, A. Z. and M. R. Eston. 2009. Mamíferos (Chordata: Mammalia) florestais de médio e grande porte registrados em Barreiro Rico, Anhembi, estado de São Paulo. Revista Instituto Florestal 21:201-215.

Aquino, E. A. C. A. 1999. Preferência alimentar de Alouatta caraya relacionada ao sexo e a idade no pantanal Miranda-Abobral, Corumbá, MS. IX Congresso Brasileiro de Primatologia.

Araujo, A. 2002. Volume testicular, idade e status reprodutivo em Callithrix jacchus (Callitrichidae, Primates) selvagens.in X Congresso Brasileiro de Primatologia.

Araújo, A. 1997. Características dos locais de dormida de Callithrix jacchus em ambiente natural.in VIII Congresso Brasileiro de Primatologia, João Pessoa PB- Brazil.

Araújo, A. and M. F. Arruda. 1997. Sucessão de pares reprodutores em grupos de Callithrix jacchus em ambiente natural.in VIII Congresso Brasileiro de Primatologia e V Reunião Latinoamericana de Primatologia, João PessoaBrazil.

Araújo Jr., A. C., U. R. Gomes, J. J. Didonet, M. V. R. Soares, C. S. Araújo, P. Saletti, and V. F. Pessoa. 2007. Aprendizagem em discriminação de cores no bugio preto (Alouatta caraya ).in XII Congresso Brasileiro de Primatologia.

Araujo, R. M., V. Sabatini, and C. R. Ruiz-Miranda. 2005. Análise bioacústica da vocalização de longa distância de saguis (Callithrix jacchus e Callithrix penicillata) sob efeitos da fragmentação florestal.in XI Congresso Brasileiro de Primatologia.

Araújo, R. M., M. B. Souza, and C. R. Ruiz-miranda. 2005. Densidade Populacional de Cebidae (Alouatta guariba guariba e Cebus apella nigritus) em remanescentes de Mata Atlântica do norte do estado do Rio de Janeiro.in XI Congresso Brasileiro de Primatologia, Porto Alegre- Brazil.

Arins, F. O., A. L. Roveda, H. G. Matias, and S. S. Dornelles. 2005. Padrão de utilização do espaço por um grupo de bugios Alouatta gariba clamitans (CABRERA,1940) (Primates, Atelidae), em São Francisco do Sul - SC.in XI Congresso Brasileiro de Primatologia, Porto Alegre- Brazil.

Arruda, M. F., A. Araújo, M. B. C. Sousa, F. A. Albuquerque, A. C. S. R. Albuquerque, and M. E. Yamamoto. 2002. Reprodução ocasional de fêmeas subordinadas silvestres de Callithrix jacchus: monogamia ou poliginia?in $\mathrm{X}$ Congresso Brasileiro de Primatologia.

Atlântica, I. d. P. d. M. 2005. Conservação da Mata Atlântica no estado do Espírito Santo: cobertura florestal, unidades de conservação e fauna ameaçada. Programa Centros para a Conservação da Biodiversidade. Conservação Internacional do Brasil/ IPEMA, Vitória- Brazil.

Ayres, M. J., I. J. Bonsiepe, and T. J. Townshend. 1989. Notes on monkeys and habitat in the northeast of Marajó Island, Brazil. Primate Conservation 10:21-22.

Azevedo, R. B. and J. C. Bicca-Marques. 2007. Posturas de alimentação e tipos de locomoção utilizados por bugios-ruivos (Alouatta guariba clamitans Cabrera, 1940). Pages 375-385 in J. C. Bicca-Marques, editor. A Primatologia no Brasil. SBPr, Porto Alegre - Brazil.

Balestra, R. and R. P. Bastos. 1999. Social interactions among tufted capuchins (Cebus apella) in an area of anthropic influence.in IX Congresso Brasileiro de Primatologia.

Barbosa, E. F., L. G. Dias, L. S. Moreira, and F. R. Melo. 2007. Influência do muriquido-norte, Brachyteles hypoxanthus (Primates, Atelidae) no comportamento de 
outros animais no Parque Estadual da Serra do Brigadeiro.in XII Congresso Brasileiro de Primatologia. SBPr, Belo Horizonte- Brazil.

Bardier, G. and D. Santee. 1999. Callithrix jacchus (L. 1758): Competição ou coexistência pacífica?in IX Congresso Brasileiro de Primatologia.

Barreto, C. E. and M. F. Arruda. 1997. Perfil da interação social de fêmeas reprodutivas de Callithrix jacchus (Primates: Callitrichidae) em grupos poligínicos no ambiente natural.in VIII Congresso Brasileiro de Primatologia e V Reunião Latino-americana de Primatologia, João Pessoa- Brazil.

Barros, R. S. M. 2008. Levantamento e estimativas populacionais de mamíferos de médio e grande porte num fragmento de Mata Atlântica em área urbana no sudeste do Brasil.

Bastazini, V. A. G. 2011. Efeitos da estrutura de habitat e do espaço sobre a diversidade de mamíferos no norte do Pantanal: uma abordagem de resolução fina.

Bastos-Neto, O. J., E. G. R. de Oliveira, D. P. Souza, B. F. Mello, T. O. S. Amorim, K. C. P. Gomes, and A. Andriollo. 2009. Mamíferos de um fragmento florestal particular periurbano de Juiz de Fora, Minas Gerais, Brasil. Revista Brasileira de Zoociências 11:269-276.

Batista-Morais, N., B. Neilson-Rolim, H. H. Matos-Chaves, J. De Brito-Neto, and L. Maria-da-Silva. 2000. Rabies in tamarins (Callithrix jacchus) in the State of Ceara, Brazil, a distinct viral variant? MEMORIAS DO INSTITUTO OSWALDO CRUZ.

Bezerra, B. M. and A. S. Souto. 2005. Uso preferencial das mãos pelo sagui comum (Callithrix jacchus) em testes envolvendo problema com fios paralelos em seu ambiente natural.in XI Congresso Brasileiro de Primatologia.

Bezerra, B. M. and A. S. Souto. 2008. Structure and usage of the vocal repertoire of Callithrix jacchus. International Journal of Primatology 29:671-701.

Bicca-Marques, J. C. and C. Calegaro-Marques. 1994. Activiy budget and diet of Alouatta caray: an age-sex analysis. Folia Primatologica 63:216-220.

Bicca-Marques, J. C., H. M. Prates, F. R. C. De Aguiar, and C. B. Jones. 2008. Survey of Alouatta caraya, the black-and-gold howler monkey, and Alouatta guariba clamitans, the brown howler monkey, in a contact zone, State of Rio Grande do Sul, Brazil: evidence for hybridization. Primates 49:246-252.

Bobadilla, U. L. and S. F. Ferrari. 1997. Evaluation of the populations of Chiropotes satanas utahicki at two sites en eastern Amazonia.in VIII Congresso Brasileiro de Primatologia e V Reunião Latino Americana de Primatologia.

Boere, V., L. Tilmann, M. C. De Resende, and C. Tomaz. 2000. Uso do espaco e comportamento social de saguis do cerrado (Callithrix penicillata) selvagens, no centro de primatologia da Universidade de Brasília.in C. Alonso and A. Langguth, editors. A PRIMATOLOGIA NO BRASIL. SBPr.

Bonvicino, C. R. 1989. Ecologia e comportamento de Alouatta belzebul (Primates: Cebidae) na Mata Atlântica. Revista Nordestina de Biologia 6:149-179.

Bonvicino, C. R., A. Langguth, and R. A. Mittermeier. 1989. A study of pelage color and geographic distribution in Alouatta belzebul (Primates: Cebidae). Revista Nordestina de Biologia 6:139-148.

Briania, D. C., R. T. Santori, M. V. Vieira, and N. Gobbi. 2001. Mamíferos nãovoadores de um fragmento de mata mesófila semidecídua, do interior do Estado de São Paulo, Brasil. Holos Environment:141-144.

Brigido, M. C., H. S. Ferreira, J. A. Muniz, M. A. Costa, and V. J.H. 1997. Dados hematológicos em animais da espécie Alouatta caraya, oriundos do resgate de 
fauna da área de influência do lago da UHE - Serra da Mesa - Goiás (Primates: Atelidae).in VIII Congresso Brasileiro de Primatologia.

Burity, C. H. F., L. D. Cruz, V. L. Rocha, N. B. Conceição, D. E. Luz, D. S. Santos, D. C. Campos, and A. Pissinatti. 2007. Golden lion tamarins, Leontopithecus rosalia (Linnaeus, 1766) in the Taquara Municipal Natural Park (Duque de Caxias, RJ): a southern extension of the known range. Neotropical Primates 14:30-31.

Buss, G. and H. P. Romanowski. 2002. Estudo preliminar do monitoramento do bugio ruivo, Alouatta guariba clamitans, através da contagem de bolos fecais no Parque Estadual de Itapuã, Rio Grande do Sul, Brasil. . Neotropical Primates 10:76-79.

Cabral, A., A. F. O. Fermoseli, T. Pereira, and W. F. Santos. 2005a. Fissão de um bando de bugios-pretos (Alouatta caraya)em mata urbana de Ribeirão Preto-SP.in XI Congresso Brasileiro de Primatologia.

Cabral, A., A. F. O. Fermoseli, T. Pereira, and W. F. Santos. 2005b. Relato de interações agressivas entre Alouatta caraya em fragmentos de mata remanescentes na região de Ribeirão Preto-SP.in XI Congresso Brasileiro de Primatologia.

Calegaro-Marques, C. and J. C. Bicca-Marques. 1997a. Comportamento agressivo em um grupo de bugios-pretos, Alouatta caraya (Primates, Cebidae). A Primatologia no Brasil.

Calegaro-Marques, C. and J. C. Bicca-Marques. 1997b. Vocalizações de Alouatta caraya (Primates, Cebidae). A Primatologia no Brasil.

Camargo, C. C. and S. F. Ferrari. 2007a. Interactions between tayras (Eira barbara) and red-handed howlers (Alouatta belzebul) in eastern Amazonia. Primates 48:147150.

Camargo, C. C. and S. F. Ferrari. 2007b. Observations of daytime births in two groups of red-handed howlers (Alouatta belzebul) on an island in the Tucurui Reservoir in eastern Brazilian Amazonia. American Journal of Primatology 69:1075-1079.

Camargo, C. C. C. 2008. Ecologia e Comportamento do Macaco-Caiarara (Cebus kaapori). . Museu Paraense Emílio Goeldi.

Camargo, C. G., A. Langguth, and S. Porfírio. 2002. Variações dos padrões de atividades diárias do Guariba, Alouatta Belzebul, de acordo com a precipitaçlão e após Intervalo de 13 anos.in X Congresso Brasileiro de Primatologia. Sociedade Brasileira de Primatologia, Belém- PA.

Camaroti, F. L. M. and M. A. B. Oliveira. 2007. Atos de predação no local de pernoite de Callithrix jacchus por gaviões-carijós (Rupornis magnirostris).in XII Congresso Brasileiro de Primatologia.

Camarotti Seal, F. L. M. and M. A. O. M. Da Cruz. 1997. Fatores ecológicos e comportamentais implicados na seleção e uso dos locais de pernoite de grupos de Callithrix jacchus em ambiente natural.in VIII Congresso Brasileiro de Primatologia.

Camillo, C. S., C. V. M. Azevedo, L. B. Bezerra, and J. P. S. Macedo. 1997. Efeito do estado reprodutivo sobre o padrão diário da atividade locomotora na fêmea de Callithrix jacchus mantida em cativeiro.in VIII Congresso Brasileiro de Primatologia.

Canale, G. R., L. C. Gondim, and D. P. Santee. 2002a. Observação de marcação esternal em Callithrix penicillata.in X Congresso Brasileiro de Primatologia.

Canale, G. R., D. P. Santee, L. Gondim, T. F. Pianta, D. Sampaio, L. M. Almeida, I. O. Silva, and V. Boere. 2002b. Novos registros de Callithrix penicillata 
(Callitrichidae, Primates) na região sudoeste de Goiás.in X Congresso Brasileiro de Primatologia.

Carmignotto, A. P. and C. C. Aires. 2011. Mamíferos não voadores (Mamalia) da Estação Ecológica Serra Geral do Tocantins. Biota Neotropica 11:307-322.

Carminatti, M. O. F. and P. Izar. 2007. Initial interactions among infants in tufted capuchin monkeys (Cebus apella).in XII Congresso Brasileiro de Primatologia.

Carminatti, M. O. F. and E. Z. F. Setz. 2005. Desenvolvimento de dois infantes de bugio preto Alouatta caraya em cativeiro.in XI Congresso Brasileiro de Primatologia.

Carneiro, R. S., J. A. G. Da Silva, and M. A. F. Andrade e Silva. 1999. Ocorrência de seis casos de albinismo em um grupo familiar de Callithrix jacchus (Linnaeus, 1758) no município de Limoeiro-PE-BR.in IX Congresso Brasileiro de Primatologia.

Carvalho-Junior, O. '2003. Primates in a forest fragment in eastern Amazonia. Neotropical Primates 24:424-431.

Carvalho, A. C. T., E. O. Cisalpino, A. F. P. De Castro, M. B. Serafim, and L. H. Pereira. 1991. Escherichia coli in marmosets, Callithrix penicillata and C. geoffroyi (Callitrichidae, Primates).in A. B. Rylands and A. T. Bernardes, editors. A Primatologia no Brasil. SBPr, Belo Horizonte- Brazil.

Cäsar, C., R. Byrne, R. J. Young, and K. Zuberbuhler. 2012. The alarm call system of wild black-fronted titi monkeys, Callicebus nigrifrons. . Behavioral ecology and sociobiology 66:653-667.

Caselli, C. B. and E. Z. F. Setz. 2011. Feeding ecology and activity pattern of blackfronted titi monkeys (Callicebus nigrifrons) in a semideciduous tropical forest of southern Brazil. . Primates 52:351-359.

Cassimiro, R. A., N. Freitas, and A. Araujo. 2002. Influência das atividades diárias na utilização de área de uso em grupos de Callithrix jacchus (Primates: Callitrichidae).in X Congresso Brasileiro de Primatologia.

CASTRO, C. S. S. 2002. Técnicas de germinação utilizadas em sementes dispersadas, pelo sagui (Callithrix jacchus), num fragmento de Mata Atlântica.in X Congresso Brasileiro de Primatologia.

Castro, C. S. S., A. Araujo, M. M. Dias-Filho, and C. Alho. 1997. Influência da distribuição espaço-temporal de frutos na dieta e no padrão da área do sagui (Callithrix jacchus).in VIII Congresso Brasileiro de Primatologia.

Castro, C. S. S., A. A. L. Menezes, J. W. Queiroz, and L. F. S. Moreira. 1994. Estudo dos ritmos biológicos da catação no sagui comum (Callithrix jacchus) em ambiente natural.in M. E. Yamamoto and M. B. C. Sousa, editors. A Primatologia no Brasil. SBPr.

Castro, C. S. S., D. M. R. Silva, F. A. Costa Junior, P. H. G. Angeiras, M. F. Souza, R. A. M. Torres, W. F. Alves, and P. G. Souza. 2007. Censo populacional do sagüi (Callithrix jacchus) em remanescente de Mata Atlântica.in XII Congresso Brasileiro de Primatologia.

Chabrawi, S., F. Caixeta, V. Pessoa, and M. Araújo. 2007. Caracterização cromática das fontes alimentares de um grupo de bugio-preto (Alouatta caraya - Primates. XII Congresso Brasileiro de Primatologia.

Chagas, R. R. D. and S. F. Ferrari. 2010. Habitat use by Callicebus coimbrai (Primates: Pitheciidae) and sympatric species in the fragmented landscape of the Atlantic Forest of southern Sergipe, Brazil. Zoologia (Curitiba, Impresso) 27:853-860.

Chiarello, A. G. 2002. Primates of the Brazilian Atlantic Forest: The Influence of Forest Fragmentation on Survival. Pages 99-118 in L. K. Marsh, editor. Primates in 
Fragments - Ecology and Conservation. Kluwer Academic/ Plenum Publishers, New York, USA.

Chiarello, A. G. and F. R. Melo. 2001. Primate population densities and sizes in Atlantic Forest remnants of northern Espirito Santo, Brazil. International Journal of Primatology 22:379-396.

Chiarello, A. G. and M. Passamani. 1993. A reintroduction program for Geoffroy's marmoset, Callithrix geoffroyi. Neotropical Primates 1:6-7.

Cirne, M. F. C. and H. M. Bezerra. 1997. O comportamento agonista intersexos em função do acesso ao alimento em pares de saguis( Callithrix jacchus).in M. B. C. Sousa and A. A. L. Menezes, editors. A Primatologia no Brasil. SBPr, NatalRN- Brazil.

Codenoti, T. L., V. M. Silva, V. J. Albuquerque, E. V. Camargo, and R. M. M. Silveira. 2002. Distribuição e situação atual de Conservação de Alouatta caraya (Humboldt, 1812) no Rio Grande do Sul, Brasil Neotropical Primates:132-141.

Codenotti, T. L., L. Betanin, V. J. Albuquerque, L. Ferrari, and B. S. Silva. 2005. Ocorrência do bugio-ruivo (Alouatta guariba clamitans) na região dos Campos de Cima da Serra, Rio Grande do Sul. Page 87 in XI Congresso Brasileiro de Primatologia.

Codenotti, T. L., V. M. Da Silva, V. J. Albuquerque, E. V. Camargo, and R. M. M. Silveira. 2002. Distribuição e densidade populacional de Alouatta caraya (HUMBOLDT, 1812) (Primates, Atelidae) no Rio Grande do Sul.in X Congresso Brasileiro de Primatologia.

Coimbra-Filho, A. F. 1984. Situação atual dos calitriquídeos que ocorrem no Brasil (Callithricidae - Primates).in M. T. Melo, editor. A Primatologia no Brasil. Sociedade Brasileira de Primatologia, Belo Horizonte.

Coimbra-Filho, A. F., I. G. Câmara, and A. B. Rylands. 1995. On the geographic distribution of the Red-Handed Howling Monkey, Alouatta belzebul, in NorthEast Brazil. Neotropical Primates 3:176-178.

Coimbra-Filho, A. F., A. B. Rylands, A. Pissinati, and I. B. Santos. 1991a. The distribution and status of the buff-headed capuchin monkey, Cebus xanthosternos Wied 1820, in the Atlantic Forest region of eastern Brazil Primate Conservation 12-13:24-30.

Coimbra-Filho, A. F., R. R. Silva, and A. Pissinati. 1991b. Acerca da distribuição geográfica original de Cebus apella xanthosternos Wied, 1820 (Cebidae, Primates). A Primatologia no Brasil. Sociedade Brasileira de Primatologia, Belém- PA- Brazil.

Colombini, F. R. X., L. M. Scoss, and F. R. Melo. 2005. Tamanho de um grupo de Cebus nigritus (Primates: Cebidae) na área de uso intensivo do Parque Estadual do Rio Doce, MG.in XI Congresso Brasileiro de Primatologia.

Consenza, B. A. P. and F. R. Melo. 1998. Primates of the Serra do Brigadeiro State Park, Minas Gerais, Brazil. Neotropical Primates 6:18-20.

Cormier, L. 2000. Cultural practices benefitting primate conservation among the Guajá of eastern Amazonia. Neotropical Primates 11:100-103.

Corrêa, H. K. M., P. E. G. Coutinho, and S. F. Ferrari. 2002. Diet of Mico argentatus groups in natural forest fragments of Alter do Chao, Santarem, Para.in XI Congresso Brasileiro de Primatologia.

Corrêa, H. K. M., P. E. G. Coutinho, and S. F. Ferrari. 2007. Seasonal variation in activity patterns of groups of Mico argentatus in a natural forest fragment in Alter do Chao, Santarem, Para.in XII Congresso Brasileiro de Primatologia. 
Cosenza, B. A. P. and F. R. Melo. 1998. Primates of the serra do Brigadeiro State Park, Minas Gerais, Brazil. . Neotropical Primates. 6:18-20.

Costa-Araújo, R., A. Schuler-da-Silva, L. V. Astarita, and J. C. Bicca-Marques. 2007. Variações temporais na germinação de sementes de Ficus organensis (miq.) miq. ingeridas por um bando de bugios-ruivos (Alouatta guariba clamitans Cabrera, 1940).in XII Congresso Brasileiro de Primatologia. SBPr, Belo HorizonteBrazil.

Coutinho, J. F. V., I. D. Lima, A. M. T. Silva, M. S. B. Freire, W. M. Bonfim, V. L. R. S. Barros, P. F. C. Vasconcelos, E. Hoefer, M. G. L. De Queiroz, G. B. Araujo, J. A. P. C. Muniz, R. A. Carvalho, I. C. Da costa, and M. F. De souza. 2007. Investigação etiológica de uma epizootia em saguis (Callithrix jacchus) numa área para febre amarela.in J. C. Bicca-Marques, editor. A Primatologia no Brasil. SBPr, Porto Alegre- Brazil.

Cunha, A. A. 2005. Estratificação vertical do macaco-prego (Cebus sp.) e do sagui-detufos-brancos (Callithrix jacchus) no Maciço da Tijuca, Rio de Janeiro, RJ, Brasil.in XI Congresso Brasileiro de Primatologia.

Cutrim, F. H. R., M. D. P. Ribeiro, and M. F. Arruda. 2007. Transferência de alimento em um grupo de Callithrix jacchus como parte do cuidado à prole na Floresta Nacional de Assu-RN.in XII Congresso Brasileiro de Primatologia.

Da Cunha, R. G. T. and R. W. Byrne. 2007. The screech complex of calls of immature black howler monkeys (Alouatta caraya). XII Congresso Brasileiro de Primatologia.

Da Silva, G. S. AND M. A. O. Monteiro da Cruz. 1993. Comportamento e composição de um grupo de Callithrix jacchus Erxleben (Primates, Callithrichidae) na mata de dois Irmãos, Recife, Pernambuco, Brasil. Revista Brasileira de Zoologia.

Da Silva, J. A. G. 1999. Determinação da área de uso de um grupo de saguis Callithrix jacchus (Primates: Callithrichidae) no campus do Pici-UFC.in IX Congresso Brasileiro de Primatologia.

Da Silva, R. M., J. C. Bicca-Marques, and H. D. Ferreira. 1996. Dados preliminares sobre a dieta do bugio Alouatta caraya (Primates, Cebidae) na área do Parque Zoológico de Goiânia - PZG.in XXI Congresso Brasileiro de Zoologia.

Da Silva, S. S. B. 2003. Comportamento alimentar do cuxiú-preto (Chiropotes satanas) na área de influência do reservatório da usina hidrelétrica de Tucuruí-Pará.

Da Silva, V. M. and T. L. Codenotti. 2007. Mapeamento das áreas de ocorrência de Alouatta caraya em fragmentos florestais de Tupanciretã, Rio Grande do Sul.in J. C. Bicca-Marques, editor. A Primatologia no Brasil. SBPr, Porto AlegreBrazil.

Dame, D. V. and C. A. Souza. 2005. Coexistência e frugivoria de Alouatta guariba clamitans e Carponis cucullatu s sobre os frutos da espécie-chave Schefflera morototoni em um fragmento de mata no município de Camaquã. Page 95 in XI Congresso Brasileiro de Primatologia.

De Faria, D. S. 1984a. Aspectos gerais do comportamento de Callithrix jacchus penicillata em mata ciliar do cerrado.in M. T. Melo, editor. A Primatologia no Brasil. SBPr, Belo Horizonte- Brazil.

De Faria, D. S. 1984b. Uso de àrvores gomíferas do cerrado por Callithrix jacchus penicillata.in M. T. Melo, editor. A Primatologia no Brasil. SBPr, Belo Horizonte- MG- Brazil.

De Marques, A. A. B., M. Schneider, and C. J. R. Alho. 2002. Radiotelemetry monitoring of Mico melanurus (E. Geoffroy in Humboldt, 1812) translocated by the Manso Reservoir, Mato Grosso.in X Congresso Brasileiro de Primatologia. 
De Medeiros, L. N. and C. S. S. De Castro. 2007. Substituição de indivíduos reprodutores em um grupo de sagui (Callithrix jacchus) em ambiente natural.in J. C. Bicca-Marques, editor. A Primatologia no Brasil. SBPr, Porto Alegre-RSBrazil.

De Melo, A. L. and L. H. Pereira. 1986. Sobre o parasitismo por Primasubulura jacchi em Callithrix penicillata (Primates, Callitrichidae).in M. T. Melo, editor. A Primatologia no Brasil. SBPr, Belo Horizonte- Brazil.

de Melo Júnior, T. A. and J. Z. Fernando. 2007. Black-Tufted-Ear Marmoset Callithrix penicillata(Primates: Callitrichidae) Following the Army ant Labidus praedator(Formicidae: Ecitoninae) in the Cerrado and the Atlantic Forest, Brazil. Neotropical Primates 14:32-33.

De Melo, W. F. and G. A. Damasceno JR. 1999. Características de habitat de bugiospretos (Alouatta caraya) da Baia Negra, do município de Corumbá.in IX Congresso Brasileiro de Primatologia.

De Resende, D. M., L. H. Pereira, A. L. De Melo, W. L. Tafure, N. I. B. Moreira, and C. L. Oliveira. 1994. Parasitism by Primasubulura jacchi (Marcel, 1857) Inglis, 1958 and Trichospirura, leptostoma Smith and Chitwood, 1967 in Callithrix penicillata marmosets, trapped in the wild environment and maintained in captivity. Memorias do Instituto Oswaldo Cruz.

de Sá, R. M. L. 1991. A populaçõa de Brachyteles arachnoides (Primates, cebidae) da Fazenda Esmeralda, Rio Casca, Minas Gerais. Pages 235-238 in A. B. Rylands and A. T. Bernardes, editors. A Primatologia no Brasil. SBPr, Belo Horizonte Brazil.

De Sá, R. M. L., T. R. Pope, K. E. Glander, T. T. Struhsaker, and G. A. B. Da Fonseca. 1990. A pilot study of genetic and morphological variation in the muriqui (Brachiteles arachnoides). Primate Conservation 11:26-30.

Decanini, D. P. and R. H. Macedo. 2008. Sociality in Callithrix penicillata: I. Intragroup Male Profile. International Journal of Primatology 29:433-447.

Dias, L. G. and K. B. Strier. 2000. Agonistic encounters between muriquis, Brachyteles arachnoides hypoxanthus (Primates, Cebidae), and other animals at the Estação Bioloógica de Caratinga, Minas Gerais, Brazil. Neotropical Primates 8:138-141.

Diego, V. H., S. F. Ferrari, and F. D. C. Mendes. 1993. Conservação do Sagüi-da-Serra (Callithrix flaviceps) o papel de matas particulares. Pages 205-206 in M. E. Yamamoto and M. B. C. Sousa, editors. A Primatologia no Brasil. SBPr, Belo Horizonte - Brazil.

Digby, L. J. 1998. Vertebrate predation in common marmosets. Neotropical Primates 6:124-126.

Digby, L. J., S. F. Ferrari, and A. A. J. F. Castro. 1996. Preliminary records of common marmosets (Callithrix jacchus) from the Sete Cidades National Park, Piaui, Brazil. Neotropical Primates 4:53-55.

Duque, E. F. and S. P. Bravo. 1997. Population genetics and conservation of owl monkeys (Aotus azarai) in Argentina: a promising field site. Neotropical Primates 5 48-50.

Duque, E. F., C. P. Juaréz, and A. Di Fiore. 2008. Adult male replacement and subsequent infant care by male and siblings in socially monogamous owl monkeys (Aotus azarai). Primates 49:81-84.

Evaristo, G. H., F. Beilke, and S. S. Dornelle. 2009. Censo e análise da situação dos primatas encontrados na APA Serra Dona Francisca, Joinville/SC.in XIII Congresso Brasileiro de Primatologia. 
Faulkes, C. G., M. F. Arruda, and A. O. Monteiro da Cruz. 2003. Matrilineal genetic structure within and among populations of the cooperatively breeding common marmoset, Callithrix jacchus. Molecular ecology 12:1101-1108.

Fermoseli, A. F. O., A. Cabral, F. R. Tognon, T. S. Pereira, and W. F. Santos. 2005a. Relações de tamanho de grupo com dinâmica populacional em bugios pretos (Alouatta caraya) em regime de semi-cativeiro no município de Ribeirão Preto/SP.in XI Congresso Brasileiro de Primatologia.

Fermoseli, A. F. O., O. G. Marne, C. C. Minei, T. S. Pereira, F. R. Tognon, A. Cabral, and W. F. Santos. 2005b. Efeitos da ação antrópica sobre o padrão de atividades diárias e o tamanho do grupo de bugios Alouatta caraya (Primates, Cebidae) em regime de semi-cativeiro em Barrinha, SP.in XI Congresso Brasileiro de Primatologia.

Ferrari, S. F. 1991. Preliminary Report on a Field Study of Callithrix flaviceps. Pages 159-171 in A. B. Rylands and A. T. Bernardes, editors. A Primatologia no Brasil. Sociedade Brasileira de Primatologia, Belo Horizonte- Brazil.

Ferrari, S. F., U. L. Bobadilla, and C. Emidio-Silva. 2007. Where have all the Titis gone? The heterogeneous distribution of Callicebus moloch in Eastern Amazonia, and its implications for the conservation of Amazonian primates. Primate Conservation 22:49-54.

Ferrari, S. F., S. Iwanaga, M. R. Messias, E. M. Ramos, P. C. S. Ramos, E. H. C. CruzNeto, and P. E. G. Coutinho. 2000. Titi Monkeys (Callicebus spp., Atelidae: Platyrrhini) in the Brazilian State of Rondônia. Primates. 41:229-234.

Ferreira, R. G., L. Jerusalinsky, T. C. F. Silva, M. S. Fialho, A. A. Roque, A. Fernandes, and F. Arruda. 2009. On the occurrence of Cebus flavius (Schreber 1774) in the Caatinga, and the use of semi-arid environments by Cebus species in the Brazilian state of Rio Grande do Norte. Primates 50:357-362.

Fialho, M. S. 2010. Contribuição à distribuição no gênero Mico (Callitrichidae, Primates) no médio Teles Pires, Jacareacanga, Pará. Neotropical Primates 17:3132.

Fialho, M. S., M. M. Valença-Montenegro, T. C. Silva, J. G. Ferreira, and P. O. Laroque. 2014. Ocorrência de Sapajus flavius e Alouatta belzebul no Centro de Endemismo Pernambuco. Neotropical Primates 21:214-219.

Flaiban, K. K. M. C., K. A. H. Spohr, L. S. Malanski, W. K. Svoboda, M. M. Shiozawa, C. L. S. Hilst, F. C. Aguiar, I. T. Navarro, M. R. S. Balarin, J. A. N. L. M. Lisboa, G. Ludwig, and F. C. PASSOS. 2008. Hematologic values of freeranging Cebus cay and Cebus nigritus in southern Brazil. 29:1375-1382.

Flesher, K. 1999. Primates of the Ituberá Forest Complex, Bahia, Brazil. Neotropical Primates 7:127-131.

Florestas, I. E. d. 1994. Parque Estadual do Rio Doce: Um convite à pesquisa. Neotropical Primates 2:17-18.

Fontes, M. A. L., A. T. Oliveira-Filho, and M. Galetti. 1996. The Muriqui in the Parque estadual de Ibitipoca, Minas Gerais. Neotropical Primates 4:24-25.

Fortes, V. B. 2002. Um caso de rapto ou transferência acidental de um infante entre bandos vizinhos de Allouata guariba clamitan s. Neotropical Primates 10:122124.

Fortes, V. B., F. Koch, R. B. Azevedo, and J. C. Bicca- Marques. 2005. Comportamento de beber do bugio-ruivo, Alouatta guariba clamitans em duas áreas de estuo no Rio Grande do Sul. Page 102 in XI Congresso Brasileiro de Primatologia. 
Freitas, C. H., E. Z. F. Setz, and N. Gobbi. 2005. Home range of Cebus apella in forest fragments in the municipality of Franca, SP.in XI Congresso Brasileiro de Primatologia.

Freitas, E. B. 2010. Levantamento das Populações de Mamíferos e Aves em um Fragmento de Caatinga no Alto Sertão Sergipano. .

Fries, B. G., M. E. L. Summa, 1. 1. Silva, A. F. Magalhães, A. M. Brischi, R. G. Q. Benesi, M. K. Vasconcellos, S. Namba, V. M. R. Oliveira, M. M. P. Vicentim, H. C. Franco, M. A. Rizzo, R. G. Romano, E. A. P. Recco, R. J. F. Garcia, G. M. P. Ferreira, J. L. Summa, and V. C. Geraldi. 2007. Adaptação pré-soltura e soltura do bugio Alouatta guariba clamitans (Cabrera, 1940), (Primates, Atelidae), em áreas de mata atlântica no município de São Paulo*.in XII Congresso Brasileiro de Primatologia. SBPr, Belo Horizonte- Brazil.

Gabriel, C., M. M. Weber, J. T. Paranhos, and V. B. Fortes. 2005. Germinação de sementes de timbaúva, Enterolobium contortisiliquum (Leguminosae: Mimosoidae), consumidas pelo bugio-ruivo, Alouatta guariba clamitans (Platyrrhini: Altelidae). Page 103 in XI Congresso Brasileiro de Primatologia.

Galetti, M., F. I. Pedron, and M. Paschoal. 1994. Infanticide in the Brown howler monkey, Alouata fusca. Neotropical Primates 2:6-7.

Garcia, J. L., W. K. Svoboda, A. L. Chryssafidis, L. S. Malanski, M. M. Shiozawa, L. L. Aguiar, G. M. Teixeira, G. Ludwig, L. R. Silva, C. Hilst, and I. T. Navarro. 2005. Sero-epidemiological survey for toxoplasmosis in wild New World monkeys (Cebus spp.; Alouatta caraya) at the Paraná river basin, Paraná State, Brazil. . Veterinary parasitology 133:307-311.

Garcia, V. L. A. 2005. Status of the muriqui (Brachyteles) populations remaining in the state of Rio de Janeiro, Brazil: Projeto Muriqui-Rio. Neotropical Primates 13:73-78.

Gaspar, D. A. 2005. Comunidade de mamíferos não voadores de um fragmento de floresta Atlântica semidecídua do município de Campinas, SP. Unicamp, Campinas, SP.

Gestich, C. C. 2012. Influência da temperatura na ecologia e no comportamento de Callicebus nigrifrons (Primates: Pithecidae). . Unicamp.

Godoy, K. C. I., A. Odalia-Rimoli, and J. Rimoli. 2002. Primeiras informações sobre infestação por endoparasitas em grupo de bugios-pretos (Alouatta caraya Humboldt, 1812; Primates, Atelinae) no Estado de Mato Grosso do Sul.in X Congresso Brasileiro de Primatologia.

Gomes, D. F. and J. C. Bicca-Marques. 2007. Cognitive ecology and social foraging in black-horned capuchin monkeys, Cebus nigritus (Goldfuss, 1809).in XII Congresso Brasileiro de Primatologia, Belo Horizonte- Brazil.

Gonçalves, E. C., S. F. Ferrari, E. V. Menezes, A. Silvia, and M. P. C. Schneider. 2002. Impacto na Diversidade Genética e Viabilidade a Longo Prazo de Populaçoes de Alouatta belzebul (Primates, Plattyrrhini): Uma Análise após 17 anos da construção da Usina Hidrelétrica de Tucuruí (PARÁ).in X Congresso Brasileiro de Primatologia. Sociedade Brasileira de Primatologia, Belém - Brazil.

Gonçalves, R. F. and D. P. Santee. 1999. Comportamento de um grupo de machos de mico-estrêla (Callithrix penicillata) numa área metropolitana de Goânia, GO.in IX CONGRESSO BRASILEIRO DE PRIMATOLOGIA.

Gondim, L. C., A. B. B. Alvarenga, G. R. Canale, D. P. Santee, and F. D. C. Mendes. 2002. Gomivoria em imaturos de Callithrix penicillata.in X Congresso Brasileiro de Primatologia. 
Graeff, V. G., J. C. Bicca-Marques, and L. V. Astarita. 2007. Viabilidade e germinação de sementes de esporão-de-galo, Celtis iganaea (Jacq.) Sargent., ingeridas pelo bugio-ruivo Alouatta guariba clamitans Corera, 1940.in J. C. Bicca-Marques, editor. A Primatologia no Brasil. SBPr, Porto Alegre- Brazil.

Greco, M. V., M. A. Andrade, G. D. M. Carvalho, E. P. M. Carvalho-Filho, and C. E. Carvalho. 2004. Callithrix penicillata na dieta de Spizaetus ornatus (Aves: Accipitridae) em área de cerrado no Estado de Minas Gerais.in S. L. Mendes and A. G. Chiarello, editors. A Primatologia no Brasil. SBPr.

Gregorin, R. 2006. Taxonomia e variação geográfica das espécies do gênero Alouatta Lacépede (Primates, Atelidae) no Brasil. Revista Brasileira de Zoologia 23:64144.

Guimarães, T. M. M., J. F. V. Coutinho, D. C. L. Dantas, and J. S. Cortez. 1999. Análise comparativa dos parâmetros hematológicos entre machos e fêmeas do Callithrix jacchus.in IX Congresso Brasileiro de Primatologia.

Hershkovitz, P. 1977. Living New World Monkeys (platyrrhini): with an introduction to primates. . The Univiversity of Chicago Press, Chicago. .

Hershkovitz, P. 1990. Titis, New World monkeys of the genus Callicebus (Cebidae, Platyrrhini): A preliminary taxonomic review. Fieldiana Zoology 55:1-109.

Hilário, R. R., F. H. G. Rodrigues, A. G. Chiarello, and I. Mourthé. 2011. Can Roads Be Used as Transects for Primate Population Surveys? . Folia Primatologica 83:4755.

Hirano, Z. M. B., I. C. Correa, and D. A. G. Oliveira. 2008. Contexts of rubbing behavior in Alouatta guariba clamitans: a scent-marking role? American Journal of Primatology 70:575-583.

Hirano, Z. M. B., P. Theis, F. Robl, and G. N. Rosa. 2002. Estudo do comportamento de um casal de bugios (Alouatta guariba clamitans) reintroduzido no Parque São Francisco de Assis- Blumenau. Page 114 in X Congresso Brasileiro de Primatologia.

Hirsch, A., E. C. Landau, A. C. M. Tedeschi, and J. O. Menegheti. 1991. Estudo comparativo das espécies do gênero Alouatta Lacépède, 1799 (Platyrrhini, Atelidae) e sua distribuição geográfica na América do Sul. Pages 239-262 A primatologia no Brasil. Sociedade Brasileira de Primatologia, Belo HorizonteBrazil.

Izar, P. 2002. Dispersão de sementes por Cebus apela e Brachyteles arachnoides em área de Mata Atlântica, Parque Estadual Intervales, SP.in X Congresso Brasileiro de Primatologia. SBPr, Belém- Brazil.

IZAR, P. and R. G. FERREIRA. 2005. Socioecology of wild and provisioned capuchin monkeys (Cebus apella): a comparative analysis.in XI Congresso Brasileiro de Primatologia.

Izar, P., A. Presotto, and M. D. Fogaça. 2008. Goal-directed movements of black capuchin monkeys, Cebus nigritus, in Atlantic Forest.in Primate Eye, IPS 2008. IPS, Edinburgh, Scotland.

Izar, P., E. D. Ramos-da-Silva, B. D. de-Resende, and E. Ottoni. 2007. A case of infanticide in tufted capuchin monkeys (Cebus nigritus). Mastozoologia Neotropical 14:73-76.

Izar, P. and T. Sato. 1997. Seed dispersal by black-capped cebus (Cebus apella) at Mata Atlantica.in VII Congresso Brasileiro de Primatologia \& V Reuniao LatinoAmericana de Primatologia. 
JARDIM, M. M. A. and E. Z. F. SETZ. 2001. Group size changes in free-ranging howler monkeys (Alouatta guariba clamitans) in southern Brazil., American Journal of Primatology.

Jardim, M. M. A. and E. Z. F. Setz. 2005. Programa Macacos Urbanos:Ecologia populacional de grupos de bugio-ruivos (Alouatta guariba clamitans) em fragmentos florestais no Sul do Brasil. Page 113 in XI Congresso Brasileiro de Primatologia.

Kierulff, M. C. M., G. Canale, and P. S. Gouveia. 2005. Monitoring the yellow-breasted capuchin monkey (Cebus xanthosternos) with radiotelemetry: choosing the best radio-collar. Neotropical Primates 13:32-33.

Kinzey, W. G. and M. Becker. 1983. Activity pattern of the masked titi monkey, Callicebus personatus. Primates 24:337-343.

Kobayashi, S. and A. Langguth. 1999. A new species of titi monkey, Callicebus Thomas, from northeasthn Brazil (Primates, Cebidae). Revista Brasileira de Zoologia 16:531-551.

Koch, F. and J. C. Bicca-Marques. 2004. Substrate manipulation by Alouatta guariba clamitans is solving a locomotor problem. Neotropical Primates 12:138-139.

Koch, F. and J. C. Bicca-Marques. 2007. Comportamento social de um grupo de bugios ruivos (Alouatta guariba clamitans).in XII Congresso Brasileiro de Primatologia, Belo Horizonte- Brazil.

Kowalewski, M. and G. E. Zunino. 2004. Birth Seasonality in Alouatta caraya in Northern Argentina. International Journal of Primatology 25:383-400.

Langguth, A., D. M. Teixeira, R. A. Mittermeier, and C. R. Bonvicino. 1987. The RedHanded Howler Monkey in Northeastern Brazil. Primate Conservation 8:36-39.

Laroque, P., S. Porfírio, and M. M. Oliveira. 2002. Adaptação de rádio transmissor para macho de Alouatta belzebul.in X Congresso Brasileiro de Primatologia. Sociedade Brasileira de Primatologia, Belém- Brazil.

Lazaro-Perea, C., C. T. Snowdon, and M. F. Arruda. 1999. Scent-marking behavior in wild groups of common marmosets (Callithrix jacchus). Behavioral ecology and sociobiology 46:313-324.

Leão, D. T., M. Brum, V. Lamim- Guedes, and Y. Antonini. 2007. Ecologia e comportamento de um grupo de Callicebus nigrifrons spix, 1823 residentes no Parque Estadual do Itacolomi, Ouro Preto, MG - dados preliminares.in XII Congresso Brasileiro de Primatologia.

Liesenfeld, M. V. A. 2005. Será o bugio-ruivo (Alouatta guariba) um eficiente dispersor das sementes do caquizinho-do-mato (Diospyros inconstans)? Um estudo in situ. Page 118 in XI Congresso Brasileiro de Primatologia.

Lima, A. K. M. and M. F. Arruda. 2007. Desenvolvimento de Callithrix jacchus no ambiente natural.in XII Congresso Brasileiro de Primatologia.

Lima, C. C. F., C. S. Oliveira, and A. C. S. R. Albuquerque. 2007. Comportamento sócio-sexual de machos adultos de Callithrix jacchus, em ambiente de caatinga.in XII Congresso Brasileiro de Primatologia.

Lima, E. M., S. S. Martins, P. M. Castor, E. C. Gonçalves, R. Ghilardi, and E. F. Ferrari. 2005a. Técnica de captura, biometria e ectopasitismo em populações fragmentadas de guaribas das mãos ruivas (Alouatta belzebul) em Tucuruí, Pará , Brasil.in XI Congresso Brasileiro de Primatologia, Porto Alegre - Brazil.

Lima, E. M., S. S. Martins, P. M. Castro, E. C. Gonçalves, R. Ghilardi, and E. F. Ferrari. 2005b. Técnica de captura, biometria e ectoparasitismo em populações fragmentadas de guaribas das maoes ruivas (Alouatta belzebul) em Tucuruí, 
Pará, Brasil.in XI Congresso Brasileiro de Primatologia, Porto Alegre- RSBrazil.

Limeira, V. L. A. 2000. Uso do espaço por um grupo de Alouatta fusca clamitans em um fragmento degradado de Floresta Atlântica. A Primatologia no Brasil.

Lokschin, L. X., F. P. Paim, J. N. H. Cabral, R. S. Rossato, R. B. SETUBAL, A. C. Alonso, F. E. Silva, G. Buss, and H. P. Romanowski. 2005. Programa Macacos Urbanos: ocorrência e distribuição do bugio-ruivo (Alouatta guariba clamirtans CABRERA 1940) em Porto Alegre, RS Etapa 2 - resultados preliminares. Page 120 in XI Congresso Brasileiro de Primatologia.

Lopes, F. A., M. F. C. Cirne, and M. E. Yamamoto. 1999. Prioridade no acesso ao alimento em machos e fêmeas reprodutivos de sagui-comum (Callithrix jacchus).in IX Congresso Brasileiro de Primatologia.

Lopes, V. S. and D. S. De Faria. 2002. Dieta do Callithrix penicillata (Primates, Callitrichidae) em áreas de cerradono Distrito Federal, Brazil. Neotropical Primates 10:17-20.

LORETTO, D. and H. RAJÃO. 2005a. Novos registros de primatas no Parque Nacional do Itatiaia, com ênfase em Brachyteles arachnoides (Primates, Atelidae).

Loretto, D. and H. Rajão. 2005b. Novos registros de primatas no Parque Nacional do Itatiaia, com ênfase em Brachyteles arachnoides (Primates, Atelidae). Neotropical Primates 13:28-29.

Lousa, T. C., K. A. Portilho, T. O. Grande, R. M. Cardoso, and F. D. C. Mendes. 2007. Feeding behavior of a semi-free ranging group of Cebus libidinosus in an experiment in a natural context.in XII Congresso Brasileiro de Primatologia.

Ludwig, G., L. M. Aguiar, and F. C. Passos. 2007. Área de vida de Alouatta caraya (Primates, Atelidae) (humboldt, 1812) em ilha e continente do alto Rio Paraná.in XII Congresso Brasileiro de Primatologia.

Ludwig, G., L. M. Aguiar, and V. J. Rocha. 2005. Uma avaliação da dieta, da área de vida e das estimativas populacionais de Cebus nigritus (Goldfuss, 1809) em um fragmento florestal no norte do Estado do Paraná. Neotropical Primates 13:1218.

Ludwig, G., L. M. Aguiar, and V. J. Rocha. 2006. Comportamento de obtenção de Manihot esculenta Crantz (Euphorbiaceae),mandioca, por Cebus nigritus (Goldfuss) (Primates, Cebidae) como uma adaptação alimentar em períodos de escassez. Revista Brasileira de Zoologia

23:888-890.

Ludwig, G., N. R. Reis, V. J. Rocha, and L. M. Aguiar. 2002. Dieta sazonal de Alouatta guariba (HUMBOLDT,1812) (Primates, Mammalia) na mata doralice, IbiporãParaná. Pages 101-121, X Congresso Brasileiro de Primatologia.

Ludwig, G., N. R. Reis, V. J. Rocha, and L. M. Aguiar. 2003. Comparação das dietas de Alouatta guariba (HUMBOLDT, 1812) e Cebus nigritus (GOLDFUSS,1809) no remanescente Mata Doralice, Ibiporã, Paraná.in II Congresso Brasileiro de Mastozoologia, Belo Horizonte- Brazil.

Magalhães, O. and C. F. Carvalho. 2004. Levantamento das espécies de Primatas da Estação Ecológica do Tripuí - Ouro Preto, MG.in XXV Congresso Brasileiro de Zoologia, Brasília.

Mannu, M. and E. B. Ottoni. 2005. Tool use by two groups of capuchins (Cebus apella) in Caatinga: preliminary data.in XI Congresso Brasileiro de Primatologia.

Maranhão, M. C. G., M. M. Oliveira, S. Porfírio, and M. A. O. Monteiro-da-Cruz. 2002. Estado de Conservação das populações de Allouatta belzebul belzebul no 
Nordeste Brasileiro.in X Congresso Brasileiro de Primatologia. Sociedade Brasileira de Primatologia, Belém- PA.

Marques, A. A. B. 2002. Use of space by Alouatta guariba clamitans Cabrera, 1940, in temperate and subtropical habitats in southern Brasil.31.

Marques, A. A. B., M. Schneider, and C. J. R. Alho. 2011. Translocation and radiotelemetry monitoring of black-tailed marmosets, Callithrix (Mico) melanura (É. Geoffroy in Humboldt), in a wildlife rescue operation in Brazil. . Brazilian journal of biology 71:983-989.

Martinez, J. and R. Wallace. 2007. Further Notes on the Distribution of Endemic Bolivian Titi Monkeys, Callicebus modestus and Callicebus olallae. . Neotropical Primates 14: 47-54.

Martinez, J. and R. B. Wallace. 2013. New information about the distribution of Callicebus (Pithecidae, Primates) in northern Beni Department, Bolivia. . Ecología en Bolivia. 48:57-62.

Martins, E. S., J. M. Ayres, and M. B. Ribeiro do Valle. 1988. On the status of Ateles belzebuth marginatus with notes on other primates of the Iriri River Basin. Primate Conservation 9:87-91.

Martins, J. R., E. L. Salomão, R. L. Doyle, M. C. Teixeira, V. C. Onofrio, and D. M. Barros-Batesti. 2006. First record of Amblyomma aureolatum (Pallas, 1772), (Acari: Ixodidae) parasitizing Allouata guariba (Humboldt, 1812) (Primata: Atelidae) in southern Brasil. Revista Brasileira de Parasitologia Veterinária 15:203-205.

Martins, M. M. 2005. Density of primates in four semi-deciduous forest fragments of Sao Paulo, Brazil. Biodiversity and Conservation 14:2321-2329.

Martins, M. M., S. F. Ferrari, and C. S. Silvia. 2002. Fragmentação de Hábiat e parasitismo em populações de Alouatta belzebul (Platyrrhini, Atelidae ) na Amazônia Oriental.in X Congresso Brasileiro de Primatologia, Belém- Brazil.

Martins, M. M. and E. Z. F. Sertz. 2000. Diet of buffy tufted-eared marmosets (Callithrix aurita) in a forest fragment in southeastern Brazil. International Journal of Primatology 21:467-476.

Matias, H. G., A. L. Roveda, F. O. Arins, E. L. Costa, S. S. Domelles, and J. C. J. Melo. 2005. Dieta de Alouatta guariba clamitans, Cabrera (1940), em um fragmento de Floresta Atlântica em São Francisco do Sul, SC. Page 125 in XI Congresso Brasileiro de Primatologia.

Melo-Jr, T. A. and F. J. Zara. 2007. Black-tufted- ear Marmoset Callithrix penicillata (Primates: Callitrichidae) Following the Army Ant Labidus predator (Formicidae: Ecitoninae) in the Cerrado and the Atlantic Forest, Brazil. . Neotropical Primates 14:32-33.

Melo, F. R. 2004. Primatas e áreas prioritárias para a conservação da biodiversidade no vale do rio Jequitinhonha, Minas Gerais Primatas e áreas prioritárias para a conservação da biodiversidade no vale do rio Jequitinhonha , Minas Gerais. UFMG.

Melo, F. R., B. A. P. Consenza, D. S. Ferraz, S. L. F. Souza, M. S. Nery, and M. J. R. Rocha. 2005a. Declínio de uma população de Brachyteles hypoxanthus (Atelidae: Primates) na Fazenda Esmeralda, Rio Casca, Minas Gerais.in XI Congresso Brasileiro de Primatologia, Porto Alegre- Brazil.

Melo, F. R., B. A. P. Consenza, D. S. Ferraz, S. L. F. Souza, M. S. Nery, and M. J. R. Rocha. 2005b. The near extinction of a population of Northern Muriquis (Brachyteles hypoxanthus) in Minas Gerais, Brazil. Neotropical Primates 13:1014. 
Melo, F. R., D. F. Fontes, and A. B. Rylands. 2002a. Primatas do Vale Jequitinhonha, Minas Gerais.in X Congresso Brasileiro de Primatologia, Belém- Brazil.

Melo, F. R. d., G. Buss, M. M. d. A. Jardim, R. C. Printes, J. d. S. e. S. Júnior, M. M. d. Oliveira, J. Rímoli, and M. Talebi. 2008. Estado da arte da primatologia no Brasil.in S. F. Ferrari and J. Rímoli, editors. A Primatologia no Brasil. SBPr, Belém- PA- Brazil.

Melo, L. C. O., A. R. Mendes- Pontes, and M. A. O. Monteiro da Cruz. 2002b. Infanticídio e canibalismo em Callithrix jacchus -saguis-do- nordeste.in $\mathrm{X}$ Congresso Brasileiro de Primatologia.

Melo, L. C. O., L. A. M. Silva, and M. A. O. Monteiro da Cruz. 2002c. Atividade de forrageio na captura de presas por Callithrix jacchus em um fragmento de Mata Atlântica - PE.in X Congresso Brasileiro de Primatologia.

Melo, L. C. O., M. M. Valenca- Montenegro, A. S. Souto, and M. A. O. Monteiro da Cruz. 2002d. Seleção de recursos alimentares por Callithrix jacchus - Sagui- donordeste: Um foco sobre a Teoria da otimização.in X Congresso Brasileiro de Primatologia.

Melo, W. F. and C. A. Pedroso. 2002. Estudo preliminar da lateralidade do ato de coçar de um grupo de bugios (Alouatta caraya) em ambiente natural. X Congresso Brasileiro de Primatologia.

Mendes, S. L. 1989. Estudo ecológico de Alouatta fusca (Primates: Cebidae) na Estação Ecológica de Caratinga, MG. Revista Nordestina de Biologia 6:71-104.

Mendes, S. L. 1991. Situação atual dos primatas em reservas florestais do Estado do Espírito Santo. Pages 347-356 in A. B. Rylands and A. T. Bernardes, editors. A Primatologia no Brasil. SBPr, Belo Horizonte- Brazil.

Menezes, M. O. T. 2004. The use of date palms (Phoenix sp.) as resting and sleeping sites by Callithrix jacchus in northeastern Brazil. Neotropical Primates 12:5355.

Menezes, M. O. T. 2005. Influência da urbanização sobre uma população de Callithrix jacchus (Linnaeus, 1758) de vida livre no campus do PICI (UFC), Fortaleza, Ceará.in XI Congresso Brasileiro de Primatologia, Porto Alegre- Brazil.

Milano, M. Z. and E. L. A. Monteiro-Filho. 2009. Predation on Small Mammals by Capuchin Monkeys, Cebus cay. . Neotropical Primates 16:78-80.

Milton, K. 1984. Habitat, diet, and activity patterns of free-ranging woolly spider monkeys (Brachyteles arachnoides E. Geoffroy 1806). International Journal of Primatology 5:491-514.

Miranda, J. M. D., I. P. Bernardi, R. F. Moro-Rios, and F. C. Passos. 2006. Three years on demography of a group of Alouatta guariba clamitans Cabrera (Primates, Atelidae): growth and fragmentation. Revista Brasileira de Zoologia 23:703706.

Miranda, J. M. D., R. F. Moro-Rios, I. P. Bernardi, and F. C. Passos. 2005. Formas não usuais para obtenção de àgua por Alouatta guariba clamitans em ambiente de floresta com araucárua no Sul do Brasil. Neotropical Primates 13:21- 23.

Miranda, J. M. D. and F. C. Passos. 2004. Hábito alimentar em Alouatta guariba (Humboldt) (Primates, Atelidae) em Floresta de Araucária, Paraná, Brasil. Revista Brasileira de Zoologia 21:821-826.

Miranda, J. M. D. and F. C. Passos. 2005. Composição e dinâmica de grupos de Alouatta guariba clamitans Cabrera (Primates, Atelidae) em Floresta Ombrófila Mista no Estado do Paraná, Brasil. Revista Brasileira de Zoologia 22:99- 106.

Miranda, J. M. D., J. E. Silva- Pereira, D. M. Mellek, and F. C. Passos. 2007. Nota sobre o hábito de beber água e o consumo de macrófitas aquáticas em Alouatta 
caraya (Humboldt, 1812) na ilha Mutum, alto rio Paraná, Brasil.in XII Congresso Brasileiro de Primatologia.

Monteiro-da-Cruz, M. A. O. 2002. Fragmentação das matas do Nordeste do Brasil x plasticidade social e ambiental do Callithrix jacchus.in X Congresso Brasileiro de Primatologia, Belém - Brazil.

Monteiro-da-Cruz, M. A. O. and E. M. Santos. 1996a. Roubo e contestação envolvendo filhote(s) e adulto(s) de Callithrix jacchus na Estação Ecológica do Tapacurá, São Lourenço da Mata/PE. XXI Congresso Brasileiro de Zoologia.

Monteiro-da-Cruz, M. A. O. and E. M. Santos. 1996b. Roubo e contestação envolvendo filhote(s) e adulto(s) de Callithrix jacchus na Estação Ecológica do Tapacurá, São Lourenço da Mata/PE.in XXI Congresso Brasileiro de Zoologia.

Monteiro, R. M., F. S. Albuquerque, and M. F. Arruda. 1997. Comparação metodológica do estudo do cuidado ao filhote em um grupo silvestre de Callithrix jacchus.in VIII Congresso Brasileiro de Primatologia.

Montilha, E. O. and K. Del-Claro. 2002. Germinação de sementes de Nectandra cissiflora, defecadas pelo bugio-ruivo (Alouatta guariba -HUMBOLT, 1812). Page 115 in X Congresso Brasileiro de Primatologia.

Moreira, J. C., E. G. Manduca, P. R. Gonçalves, R. Stumpp, C. G. C. Pinto, and G. Lessa. 2008. Mammals, Volta Grande Environmental Unity, Triângulo Mineiro, states of Minas Gerais and São Paulo, Southeastern Brazil. .

Moura, A. C. A. 2007. Primate Group Size and Abundance in the Caatinga Dry Forest, Northeastern Brazil. International Journal of Primatology 28:1279-1297.

Muhle, C. B. and J. C. Bicca-Marques. 2002. Comportamento de uma fêmea adulta de Alouata guariba clamitans antes e depois de sua transferência para um recinto com enriquecimento ambiental, no Parque Zoológic de Sapucaia do Sul, Rio Grande do Sul. Page 142 in X Congresso Brasileiro de Primatologia.

Nakai, E. S. and P. Izar. 2007. Orçamento de atividades e dieta de Cebus nigritus na mata atlântica.in XII Congresso Brasileiro de Primatologia. SBPr, Belo Horizonte- Brazil.

Nakai, E. S., J. T. Taira, and P. Izar. 2005. Interactions of Cebus apella nigritus with competitors (Brachyteles arachnoids, Alouatta guariba and Nasua nasua) in the Atlantic forest Carlos Botelho State Park, SP.in XI Congresso Brasileiro de Primatologia, Porto Alegre - Brazil.

Nascimento, F. F., C. R. Bonvicino, M. M. Oliveira, M. P. C. Schneider, and H. N. Seuanez. 2008. Population genetic studies of Alouatta belzebul from the Amazonian and Atlantic forests. American Journal of Primatology 70:423-431.

Nascimento, H. G., A. S. Souto, B. M. Bezerra, and L. G. Halsey. 2005. Correlação entre os níveis de hormônios esteroidais e a Performance do sagui comum (Callithrix jacchus) em seu ambiente natural em solucionar o teste com fios paralelos: Uma questão de individualidade e aprendizado.in XI Congresso Brasileiro de Primatologia.

Nascimento, M. C. L. and M. F. Arruda. 1997. Atividades dos cuidadores em um grupo silvestre de Callithrix jacchus: fatores que as influenciam.in VIII Congresso Brasileiro de Primatologia.

Nascimento, M. C. L. and M. F. Arruda. 2000. Influência de filhotes dependentes no padrão de atividades de um grupo silvestre de Callithrix jacchus.in C. Alonso and A. Langguth, editors. A Primatologia no Brasil. Sociedade Brasileira de Primatologia, João Pessoa- Brazil. 
Nery, M. S., D. S. Ferraz, S. L. F. Souza, E. R. Rodes, and F. R. Melo. 2007. Inventário de primatas do médio rio pardo, Bahia.in XII Congresso Brasileiro de Primatologia, Belo Horizonte.

Neves, L. G. 2008. Distribuição Geográfica e Conservação ee Callithrix kuhlii (Coimbra-Filho, 1985) (Primates, Callitrichidae) no Sul da Bahia, Brasil. . Universidade Estadual de Santa Cruz.

Nogueira, C. P., A. P. Paglia, F. A. Pimenta, R. L. Dias, L. O. Martins, and W. L. Cabral. 2002. Levantamento e distribuição de sauás, Callicebus nigrifrons, e sagui-de-tufos-pretos, Callithrix penicillata em fragmentosflorestais na região de Lavras, MG.in X Congresso Brasileiro de Primatologia.

Nogueira, D. F., D. S. Ferraz, A. F. Oliveira, F. P. Tabacow, S. M. S. Amâncio, and F. R. Melo. 2010. Ocorrência de Primatas No Parque Estadual do Ibitipoca e Entorno, Estado de Minas Gerais, Brasil. Neotropical Primates 17:67-70.

Nogueira, D. F., D. S. Ferraz, A. F. Oliveira, F. P. Tabacow, S. M. Souza, and F. R. Melo. 2007. Parâmetros populacionais de um grupo de muriqui-do-norte (Brachyteles hypoxanthus) no entorno do Parque Estadual do Ibitipoca, Minas Gerais.in XII Congresso Brasileiro de Primatologia, Belo Horizonte- Brazil.

Noronha, M. A., J. S. Silva- Junior, W. R. Spironello, and D. C. Ferreira. 2008a. New Occurrence records of Maues marmoset Mico mauesi (Primates, Callitrichidae).in X Congresso Brasileiro de Primatologia.

Noronha, M. A., J. S. Silva Jr, and D. C. Ferreira. 2008b. New Occurrence Records for Mico melanurus (Primates, Callitrichidae). Neotropical Primates. 15:26-28.

Odalia-Rimoli, A., J. D. Gonçalves, and J. Rimoli. 2002. Padrão de atividade de um grupo de saguis-de-tufo-preto (Callithrix penicillata É Geoffroy, 1812; Cebidae, Callitrichinae.in X Congresso Brasileiro de Primatologia.

Odalia-Rimoli, A. and J. Rimoli. 2005. Demografia e composição de um grupo de vida livre de Callithrix penicillata em um fragmento urbano de Cerrado, Campo Grande, Mato Grosso do Sul.in XI Congresso Brasileiro de Primatologia.

Odalia-Rimoli, A. and J. Rimoli. 2007. Demografia de um grupo de saguis-de-tufopreto (Callithrix penicillata É. Geoffroy, 1812) em um fragmento urbano de Cerrado Mato Grosso do Sul, Brasil.in J. C. Bicca-Marques, editor. A Primatologia no Brasil. SBPr, Porto Alegre- Brazil.

Oliveira, D. A. G. 2005. Description of the alarm vocalization specific to loud noises in free and semi-free capuchin monkeys (Cebus apella) in the state of Sao Paulo.in XI Congresso Brasileiro de Primatologia.

Oliveira, L. C., E. M. V. C. Camara, A. Hirsch, A. M. O. Paschoal, R. M. Alvarenga, and M. G. Belamirno. 2003. Callithrix geoffroyi (Primates: Callitrichidae) and Alouatta caraya (Primates: Atelidae) in the Serra do Cipo National Park, Minas Gerais, Brazil. Neotropical Primates 11:86-89.

Oliveira, M. F. and L. Manzatti. 1996. New locations for the muriqui (Brachyteles arachnoides) in the state of São Paulo, Brasil. Neotropical Primates 4:84-85.

Oliveira, M. M. 2007. Translocação de Alouatta belzebul na Paraíba.in XII Congresso Brasileiro de Primatologia. Sociedade Brasileira de Primatologia, Belo Horizonte- Brazil.

Oliveira, M. M. and J. C. C. Oliveira. 1993. A situação dos cebídeos como indicador do estado de conservação da mata atlântica no estado da Paraíba, Brasil. Pages 155167 A primatologia no Brasil. Sociedade Brasileira de Primatologia, SalvadorBA- Brazil.

Oliveira, M. M., S. Porfírio, P. Laroque, D. Canales, and A. Langguth. 2002. Translocação de um grupo de guaribas, Alouatta belzebul, no Nordeste 
Brasileiro.in X Congresso Brasileiro de Primatologia. Sociedade Brasileira de Primatologia, Belém - Brazil.

Oliveira, S. G., H. M. Prates, M. Mentz, and J. C. Bicca-Marques. 2007. Prevalência de Bertiella sp. em um grupo de bugios-pretos (Alouatta caraya Humbolt, 1812) de vida livre.in XII Congresso Brasileiro de Primatologia.

Oliveira, V. B., A. M. Linares, G. L. C. Corrêa, and A. G. Chiarello. 2008. Predation on the black capuchin monkey Cebus nigritus (Primates: Cebidae) by domestic dogs Canis lupus familiaris (Carnivora: Canidae), in the Parque Estadual Serra do Brigadeiro, Minas Gerais, Brazil. Revista Brasileira de Zoologia 25:376-378.

Ottoni, E. B. 2005. Behavioral traditions and adaptive plasticity in capuchin monkeys (Cebus apella).in XI Congresso Brasileiro de Primatologia.

Paccagnella, S. G. 1991. Censo da população de Monos (Brachyteles arachnoides) do parque Estadual Carlos Botelho, Estado de São Paulo. Pages 225-233 in A. B. Rylands and A. T. Bernardes, editors. A Primatologia no Brasil. SBPr, Belo Horizonte.

Passamani, M. 1996. Uso de árvores gomíferas por Callithrix penicillata no Parque Nacional da Serra do Cipó. Boletim do Museu de Biologia Mello Leitão 4:2531.

Passamani, M. 2008. Densidade e tamanho de grupos de primatas na Mata Atlântica serrana do sudoeste do Espírito Santo. Revista Brasileira de Zoociência 10:2934.

Passamani, M., S. L. Mendes, and A. G. Chiarello. 2000. Non-volant mammals of the Estação Biológica de Santa Teresa, Espírito Santo, Brazil. Boletim do Museu de Biologia Mello Leitão:207-214.

Passamani, M. and A. B. Rylands. 2000. Feeding behavior of Geoffroy's marmoset (Callithrix geoffroyi) in an Atlantic forest fragment of south-eastern Brazil. Primates 41:27-38.

Passos, F. C. and J. R. Alho. 1999. Importância de diferentes substratos no comportameno de forrageio por presas do mico-leão-preto, Leontopithecus chrysopygus.in IX Congresso Brasileiro de Primatologia.

Passos, F. C., J. M. D. Miranda, L. M. Aguiar, G. Ludwig, I. P. Bernardi, and R. F. Moro-Rios. 2006. Distribuição e Ocorrência de Primatas no Estado do Paraná, Brasil. in J. C. Bicca-Marques, editor. A Primatologia no Brasil Porto Alegre.

Paula Prist, C. and G. M. Pizzutto. 2005. Feeding And Environmental Enrichment For Howler Monkeys (Alouatta guariba). Page 134 in Proceedings of the Seventh International Conference on Environmental Enrichment.

Pereira, A. P. C. P. 2002. Ecologia alimentar do cuxiú-preto (Chiropotes satanas satanas) na Fazenda Amanda, Pará. UFPA, Belém- Brazil.

Pereira, D. G. 2006. Interações entre espécies exóticas invasoras e espécies nativas: calitriquídeos no Parque Nacional da Serra dos Órgãos, RJ. Universidade Federal Fluminense, Niterói.

Pereira, L. C. M., P. A. Nicola, and M. Valduga. 2005a. Feeding on a cone of Pinus taeda by tufted capuchin monkeys (Cebus nigritus) in south Brazil.in XI Congresso Brasileiro de Primatologia. SBPr, Porto Alegre- Brazil.

Pereira, R. F., H. O. Pengel, and C. C. De Melo. 2003. O impacto ambiental da exploração florestal de subsistência na fauna de primatas. Brasil Florestal 78:6165 .

Pereira, T. S. 2005. Padrão de atividades diárias e comportamento alimentar de um grupo de bugios-pretos (Alouatta caraya Humboldt, 1812) em um fragmento de 
mata remanescente no município de Barrinhas/SP.in XI Congresso Brasileiro de Primatologia.

Pereira, T. S., O. G. Marne, C. C. Minei, A. F. O. Fermoseli, F. R. Tognon, A. Cabral, Z. M. B. Hirano, and W. F. Santos. 2005b. Uso de áreas e utilização de rotas de locomoção por um grupo de bugios pretos (Alouatta caraya Humboldt, 1812) em um fragmento de mata no município de Barrinha/SP.in XI Congresso Brasileiro de Primatologia.

Pimenta, F. E. and J. S. Silva Júnior. 2005. An update on the distribution of primates of the Tapajos-Xinggu interfluvium, Central Amazonia. Neotropical Primates 13:23-28.

Pinha, P. S. 2007. Interações Sociais em grupos de macacos-prego (Cebus libidinosus) no Parque Nacional de Brasília. . Universidade de Brasília.

Pinha, P. S., L. B. R. Pereira, R. G. P. Silva, T. F. Brisolla, and R. H. F. Macedo. 2007. Social play in Cebus libidinosus groups in the Brasília National Park.in XII Congresso Brasileiro de Primatologia.

Pinto, A. C. B., C. Azevedo-Ramos, and O. Carvalho-Junior. 2003. Activity patterns and diet of the howler monkey Alouatta belzebul in areas of logged and unlogged forest in Eastern Amazonia. Animal Biodiversity and Conservation 26:39-49.

Pinto, A. C. B., C. Azevedo-Ramos, and O. Carvalho-Junior. 2005. Guaribas (Alouatta belzebul) e a exploração madeireira na Amazônia Oriental: Algum efeito sobre seu comportamento social?in XI Congresso Brasileiro de Primatologia. Sociedade Brasileira de Primatologia, Porto Alegre - Brazil.

Pinto, L. P. S. 1994. Distribuição geográfica, população e estado de conservação do mico-leão-da-cara-dourada, Leontopithecus chrysomelas (Callitrichidae, Primates).

Pinto, L. P. S., C. M. R. Costa, K. B. Strier, and G. A. B. Fonseca. 1993. Habitat, density and group size of primates in a Brazilian Tropical forest. Folia Primatologica 61:135-143.

Podgaiski, L. R. and A. A. Biedzicki-de- Marques. 2005. Estudo da interação mãefilhote em bugio ruivo Alouatta guariba clamitans Cabrera1940 em hábitat natural no Parque de Iatapuã, RS (Atelidae, Alouattinae). Page 153 in XI Congresso Brasileiro de Primatologia.

Pontes, A. R. M. and M. A. O. Monteiro-da-Cruz. 1995. Home range intergroup transfers, and reproductive status of common marmosets Callithrix jacchus in a forest fragment in north-eastern Brazil. Primates 36:335-347.

Porfírio, S., P. Laroque, and M. M. Oliveira. 2002a. Reintrodução de um casal de guaribas, Alouatta belzebul na Reserva Biológica Guaribas, Paraíba, Brasil.in X Congresso Brasileiro de Primatologia, Belém - Brazil.

Porfírio, S., P. Laroque, M. M. Oliveira, and C. C. Camargo. 2002b. Criação de filhote de Alouatta belzebul em semi-cativeiro: um estudo de caso.in X Congresso Brasileiro de Primatologia, Belém- Brazil.

Port-Carvalho, M. and S. F. Ferrari. 2002. Uso do hábitat por Chiropotes satanas satanas e outras três espécies de primatas em fragmentos florestais no oeste do Maranhão.in X Congresso Brasileiro de Primatologia, Belém- Brazil.

Prates, H. M. and J. C. Bicca- Marques. 2007. Age-sex analysis of activity budget, diet, and positional behavior in black-and-gold howler monkeys (Alouatta caraya).in XII Congresso Brasileiro de Primatologia.

Presotto, A. and P. Izar. 2007. Padrões de movimento de Cebus nigritus no Parque Estadual Carlos Botelho.in XII Congresso Brasileiro de Primatologia. 
Price, E. C., H. M. Piedade, and D. Wormell. 2002. Population densities of Primates in a Brazilian Atlantic Forest. Folia Primatologica 73:54-56.

Printes, R. C., M. V. A. Liesenfeld, and L. Jerusalinsky. 2001. Alouatta guariba clamitans Cabrera,1940: a new southern limit of the species and for neotropical primates. Neotropical Primates 9:118-121.

Printes, R. C., M. V. A. Liesenfeld, and L. Jerusalinsky. 2002. Novo limite Sul para a distribuição de Alouatta Clamitans e dos Primatas Neotropicais.in X Congresso Brasileiro de Primatologia, Belém- Brazil.

Printes, R. C. and M. C. C. Malta. 2007. Translocação de duas fêmeas de bugio-preto (Alouatta caraya Humboldt, 1812) do lago da Hidrelétrica de Queimado, Minas Gerais, Brasil. A Primatologia no Brasil.

Pyritz, L. W., A. B. S. Büntge, S. K. Herzog, and M. Kessler. 2010. Effects of Habitat Structure and Fragmentation on Diversity and Abundance of Primates in Tropical Deciduous Forests in Bolivia. International Journal of Primatology 31:796-812.

Queiroz, J. S. G. and M. F. Arruda. 2002. Partilha de alimento em filhotes de Callithrix jacchus com restrição de acesso à fonte.in $\mathrm{X}$ Congresso Brasileiro de Primatologia.

R.M., D. L.-N., M. A. B. Oliveira, W. R. Telino- Junior, and E. M. Santos. 2007. Comportamentos interespecíficos entre Callithrix jacchus (Linnaeus) (Primates, Calithrichidae) e algumas aves da Mata Atlântica, Pernambuco, Brasil. Revista Brasileira de Zoologia 24:709-716.

Reis, M. N. B. 2012. Ecologia e comportamento de Callicebus nigrifrons em um fragmento florestal da Mata Atlântica em Campinas-SP.

Reis, P. C. and F. D. C. Mendes. 2007. Ecologia comportamental de macaco-prego, Cebus libidinosus em uma área urbana (campus samambaia da UFG): espaçamento entre indivíduos.in VIII Congresso de Ecologia do Brasil.

Ribeiro, M. D. P., F. H. R. Cutrim, and M. F. Arruda. 2007. O desenvolvimento do padrão de atividade da infância ao período juvenil em um grupo de Callithrix jacchus na Floresta Nacional de Assu-RN.in XII Congresso Brasileiro de Primatologia.

Rimoli, J. 2007. Estudo dos primatas em fragmentos urbanos de cerrado: Cebus cay (Illiger, 1815) \& Callithrix penicillata (Geofroy, 1812) em Campo Grande, Mato Grosso do Sul.in XII Congresso Brasileiro de Primatologia.

Rimoli, J., O. Fernandes-Júnior, and A. Odalia-Rimoli. 2005. Interações sociais em calitriquineos: Uma análise da brincadeira em um grupo de saguis-de-tufo-preto (Callithrix penicillata, Geoffroy, 1812, Primate, Callitrichinae) em um fragmento urbano de Cerrado, Campo Grande, Mato Grosso do Sul.in XI Congresso Brasileiro de Primatologia.

Rimoli, J., E. M. O. Valdivino, and A. Odalia-Rimoli. 2002. Orçamento de atvidades de um grupo de bugios-pretos (Alouatta caraya, Humboldt, 1812) em um fragmento de floresta em Terenos (MS).in X Congresso Brasileiro de Primatologia.

Rocha, S. A. A. and F. D. C. Mendes. 2002. Predation of a wasp's nest by Cebus libidinosus.in X Congresso Brasileiro de Primatologia.

Rocha, V. J., L. M. Aguiar, G. Ludwig, C. L. S. Hilst, G. M. Teixeira, W. K. Svoboda, M. M. Shiozawa, L. S. Malanski, I. T. Navarro, J. H. F. Mariño, and F. C. Passos. 2007. Techniques and Trap Models for Capturing Wild Tufted Capuchins. . International Journal of Primatology 28.:231- 243. 
Rosa-Filho, A. F. and U. L. Bobadilla. 2002. A importância dos cipós na dieta do bugioruivo (Alouatta guariba clamitans) em um fragmento de Mata Atlântica do Rio Grande do Sul. Page 61, X Congresso Brasileiro de Primatologia.

Rosas Ribeiro, P. F., M. L. Soares, M. S. L. Barbosa, and A. R. Mendes Pontes. 2002. Abundância de saguis, Callithrix jacchus (Callithrichidae, Primate) em framgmentos urbanos: um estudo de caso.in $\mathrm{X}$ Congresso Brasileiro de Primatologia.

Rotundo, M., E. F. Duque, and A. F. Dixson. 2005. Infant Development and Parental Care in Free-Ranging Aotus azarai azarai in Argentina. International Journal of Primatology 26:1459-1473.

Rylands, A. B. 1988. Primates of the Rio Jequitinhonha Valley, Minas Gerais, Brazil. Primate Conservation 9:100-109.

Rylands, A. B. and A. T. Bernardes. 1989. Two Priority Regions for primate Conservation in the Brazilian Amazon. Primate Conservation 10:56-62.

Rylands, A. B., A. F. Coimbra- Filho, and R. A. Mittermeier. 1993. Systematics, geographic distribuition, and some notes on the conservation status of the Callitrichidae. Marmosets and Tamarins: Systematics, Behavior and Ecology. Oxford University Press, Oxford, USA.

Sampaio, D. T. and S. F. Ferrari. 2007. Interspecific associations of Cebus apella apella and five primates in a forest fragment in Amazonia. XII Congresso Brasileiro de Primatologia, Belo Horizonte- MG- Brazil.

Sampaio, R., J. M. Pedrosa, W. F. Santos, Z. M. B. Hirano, and H. L. Gomes. 2002. Relações de espaçamento em um grupo de bugios pretos Alouatta caraya de uma mata urbana em Ribeirão Preto.in X Congresso Brasileiro de Primatologia.

Santana, O. A. and D. P. Santee. 1999. Variação do deslocamento em função do tempo nos registros mensais de um grupo de mico-estrêla (Callithrix penicillata).in IX Congresso Brasileiro de Primatologia.

Santini, M. E. L. 1986. Padrões de Atividade Diária de Alouatta caraya (Primates, Cebidae), Reintroduzido no Parque Nacional de Brasília. Page 530 in M. T. Melo, editor. A Primatologia no Brasil, Campinas- SP- Brazil.

Santos, E. M., G. R. Abreu, and M. A. O. Monteiro-da-Cruz. 1997. Flores visitadas por Callithrix jacchus (Callitrichidae, Primates) na Estação Ecológica do Tapacurá, PE: Um provável caso de polinização.in VIII Congresso Brasileiro de Primatologia.

Santos, I. B., R. A. Mittermeier, A. B. Rylands, and C. M. C. Valle. 1987. The distribution and conservation status of primates in southern Bahia, Brazil. Primate Conservation 8:126-131.

Santos, J. S., K. Leus, and L. Van Elsacker. 2004. A sighting of Muriquis (Brachyteles) in Uma Biological Reserve, Bahia, Brazil. Neotropical Primates 12:96-97.

Santos, M. V. S., M. T. Ueta, E. Z. F. Set, and R. R. Madi. 2006. Primeiro registro de nematódeos da família kathlaniidae Travassos, 1918 (cosmocercoidea), parasitando primatas neotropicais Alouatta guariba clammitans (Atelidae), na mata Ribeirão Cachoeira, Distrito de Sousas, campinas, SP, Brasil. Bioikos 20:81-86.

Santos, R. R. 2002. Ecologia de cuxiús (Chiropotes satanas) na Amazônia Oriental: Perspectivas para a conservação de populações fragmentadas.

São Bernardo, C. S. and M. Galetti. 2004. Densidade e tamanho populacional de primatas em um fragmento florestal no sudeste do Brasil. Revista Brasileira de Zoologia 21:827-832. 
Scanlon, C. E., N. R. Chalmers, and M. A. O. Monteiro da Cruz. 1988. Changes in the size, composition, and reproductive condition of wild marmoset groups (Callithrix jacchus jacchus) in North East Brazil. Primates 29:295-305.

Scanlon, C. E., N. R. Chalmers, and M. A. O. Monteiro da Cruz. 1989. Home range use and the exploitation of gum in the marmoset Callithrix jacchus jacchus. International Journal of Primatology 10:123-136.

Schiel, N., M. B. Bezerra, A. Souto, and L. Huber. 2002. Um novo método de identificação individual do Callithrix jacchus (Primates: Callitrichidae).in $\mathrm{X}$ Congresso Brasileiro de Primatologia.

Seabra, H. F., J. I. Encinas, and J. M. Felfili. 1991. Análise estrutural da mata ciliar do córrego Capetinga - DF, habitat de Callithrix jacchus penicillata 1.in Pesquisa Agropecuaria Brasileira.

Setz, E. Z. F., G. G. Leitão, Z. M. B. Hirano, M. S. Fialho, D. A. Gaspar, and V. Garcia. 2005. O bugio ruivo Alouatta guariba clamitans e a fragmentação da Floresta Atlântica. Page 52 in XI Congresso Brasileiro de Primatologia.

Sevciuc, S. M., P. O. Laroque, and M. M. Valença-Montenegro. 2007. Consolidação do repovoamento da Reserva Biológica Guaribas, Mamanguape - PB, por Alouatta belzebul belzebul, através dos métodos de translocação e soltura progressiva de indivíduos.in XII Congresso Brasileiro de Primatologia. Sociedade Brasileira de Primatologia, Belo Horizonte- Brazil.

Silva-Júnior, J. S. 2001. Especiação nos macacos-prego e Caiaras, gênero Cebus Erxleben, 1777 (Primates, Cebidae). UFRJ.

Silva-Junior, J. S. and M. A. Noronha. 1998. On a new species bare-eared marmoset, genus Callithrix Ersleben, 1777, from Central America, Brasil (Primates: Callithrichidae). Goeldiana Zoologia 21:31.

Silva-Junior, J. S., A. Nunes, and M. E. B. Fernandes. 1995. Geographic distruition of night monkeys, Aotus, in Northern Brazil: New data and a correlation. Neotropical Primates 3:72-73.

Silva, C. R. 2001. Riqueza e diversidade de mamíferos não-voadores em um mosaico formado por plantios de Eucalyptus saligna e remascescentes de floresta atlântica no município de Pilar do Sul, SP.

Silva, E. D. R., B. D. Resende, and E. B. Ottoni. 2005. Techniques of manipulating food used by the free-living group of capuchin monkeys (Cebus apella) of the Jaragua State Park: A preliminary study.in XI Congresso Brasileiro de Primatologia.

Silva, G. M. M., K. C. S. Verissimo, and M. A. B. Oliveira. 2007. Orçamento das atividades diárias de dois grupos de Callithrix jacchus em área urbana.in XII Congresso Brasileiro de Primatologia.

Silva, I. O., A. B. B. Alvarenga, and V. Boere. 2008. Occasional field observations of the predation of mice, dove and ants by black-tufted-ear marmosets (Callithrix penicillata). Neotropical Primates 15:59-62.

Silva Jr, J. S. and M. E. B. Fernandes. 1999. A northeastern extension of the distribution of Aotus infulatus in Maranhão, Brazil. Neotropical Primates. 7:76-89.

Silva, L. D. and M. Passamani. 2007. Levantamento de mamíferos de médio e grande porte em remanescentes florestais na serra do Carrapato em Lavras/MG. .in VIII Congresso de Ecologia do Brasil, Caxambu- MG.

Sousa, M. B. C. 1999. Coleta de fezes em primatas de pequeno porte vivendo em ambiente natural: uma experiência com o Callithrix jacchus.in IX Congresso Brasileiro de Primatologia. 
Sousa, M. B. C. and M. C. Pontes. 2008. Diurnal temporal variation in affiliative behaviors of common marmosets (Callithrix jacchus) males living in natural conditions.]. Revista Brasileira de Zoociência.

Souto, A., B. M. Bezerra, N. Schiel, and L. Huber. 2007. Saltatory search in free-living Callithrix jacchus: environmental and age influences. International Journal of Primatology 28:881-893.

Souza-Júnior, J. C. 2005. Levantamento de parasitas intestinais de bugios-ruivos (Alouatta guariba clamitans) mantidos em cativeiro no município de Indaial- SC. Page 168 in XI Congresso Brasileiro de Primatologia.

Souza, L. L. Dispersão de sementes por guaribas (Alouatta belzebul ) na Amazônia Oriental (Caxiuanã, Melgaço, Pará). Estação Científica Ferreira Penna - Campus de Pesquisa, Belém- Brazil.

Souza, L. L. and S. F. Ferrari. 1999. The role of red-handed howler monkeys (Alouatta belzebul) as seed dispersers in south-eastern amazônia (Ferreira Penna Scientific Station, Caxiuaná, Pará).in IX Congresso Brasileiro de Primatologia, Santa Teresa- ES- Brazil.

Souza, R. M. M. and M. F. Arruda. 1999a. Distribução do carregar e o perfil de atividades dos cuidadores em duas proles de filhote único e uma de gêmeos de Callithrix jacchus em ambiente natural.in IX Congresso Brasileiro de Primatologia.

Souza, R. M. M. and M. F. Arruda. 1999b. Uma abordagem comportamental dos custos relacionados à reprodução e ao cuidado à prole em um grupo silvestre de Callithrix jacchus.in IX Congresso Brasileiro de Primatologia.

Stallings, J. R. and J. G. Robinson. 1991. Disturbance, forest heterogeneity and primate communities in a Brazilian Atlantic Forest Park. Pages 357-368 in A. B. Rylands and A. T. Bernardes, editors. A Primatologia no Brasil. SBPr, Belo Horizonte- Brazil.

Steinmetz, S. 2001. Densidade e Conservação do Bugio (Alouatta fusca) no Parque Estadual Intervales. Neotropical Primates 9:69-73.

Steinmetz, S. 2005. Vocalizações de longo alcance como comunicação intra-grupal nos bugios (alouatta guariba). Neotropical Primates 13:11-15.

Thorington, R. W., J. C. Ruiz, and J. F. Eisenberg. 1984. A Study of a Black Howling Monkey (Alouatta caraya) Population in Northern Argentina. . American Journal of Primatology 6:357-366.

Tillman, L., M. C. Resende, V. Boere, and C. Tomaz. 1997. Migração, habituação e uso do espaço e um grupo semi- selvagem de saguis do cerrado (Callithrix penicillata).in VIII Congresso Brasileiro de Primatologia.

Tognon, F. R., A. F. O. Fermoseli, T. S. Pereira, A. Cabral, C. C. Minei, O. G. Marne, Z. M. B. Hirano, and W. F. Santos. 2005. Alguns aspectos do comportamento alimentar de um grupo de bugios pretos (Alouatta caraya, Humboldt, 1812) em um fragmento de mata no município de Jardinópolis -SP.in XI congresso brasileiro de primatologia.

Trevelin, L. C., M. Port-Carvalho, M. Silveira, and E. Morell. 2007. . Abundance, habitat use and diet of Callicebus nigrifrons (Primates, Pithecidae) in Cantareira state park, São Paulo, Brazil. . Revista Brasileira de Zoologia 24:1071-1077.

Valenca-Montenegro, M. M., J. B. De Oliveira, and M. A. O. M. Da Cruz. 2004a. Infecção natural por Trypanosoma sp. Em Callithrix jacchus de vida livre.in S. L. Mendes and A. G. Chiarello, editors. A Primatologia no Brasil. SBPr.

Valenca-Montenegro, M. M., J. B. De Oliveira, M. A. O. M. Da Cruz, L. B. G. Da Silva, and M. C. N. Botelho. 2004b. Parasitismo por ácaro (Trombiculidae, 
Ewing, 1944) em Callithrix jacchus (Linnaeus, 1758), Callitrichidae Primates.in S. L. Mendes and A. G. Chiarello, editors. A PRIMATOLOGIA nO BRASIL. SBPr.

Valenca-Montenegro, M. M., L. C. O. Melo, Y. B. M. Valle, and M. A. O. Monteiro da Cruz. 2002a. Riscos associados à urbanização de uma área de ocorrência natural de Callithrix jacchus.in X Congresso Brasileiro de Primatologia.

Valenca-Montenegro, M. M., L. C. O. Melo, Y. B. M. Valle, and M. A. O. Monteiro da Cruz. 2002b. Tétano em Callithrix jacchus de vida livre: relato de caso.in $\mathrm{X}$ Congresso Brasileiro de Primatologia.

Valença-Montenegro, M. M., M. A. O. Monteiro-da-Cruz, J. E. Neto, and L. B. Evecio. 2005. Afecções dentárias e periodontais em saguis (Callithrix jacchus Linnaeus, 1758) de vida livre do compus da UFRPE, Recife - PE.in XI Congresso Brasileiro de Primatologia.

Valenca, M. M., J. B. Oliveira, and M. A. O. Monteiro da Cruz. 1999a. Infecção natural por Trypanosoma sp. Em Callithrix jacchus de vida livre.in IX Congresso Brasileiro de Primatologia.

Valenca, M. M., J. B. Oliveira, M. A. O. Monteiro da Cruz, M. D. Cavalcanti, and M. E. P. As. 2000. Trypanoxyuris (Hapaloxyuris) callithricis (Oxyurida, Oxyuridae) in wild Callithrix jacchus (Linnaeus, 1758), in northeast Brazil. Laboratory Primate Newsletter 39:4-5.

Valenca, M. M., J. B. Oliveira, M. A. O. Monteiro da Cruz, and L. B. G. Silva. 1999b. Parasitismo por ácaro em Callithrix jacchus de vida livre na Estação Ecológica do Tapacurá Pernambuco.in IX Congresso Brasileiro de Primatologia.

Valle, C. M. C., I. B. Santos, M. C. Alves, and C. A. Pinto. 1984. Algumas observações preliminares sobre o comportamento do mono (Brachyteles arachnoides) em ambiente natural (Fazenda Montes Claros, município de Caratinga, Minas Gerais, Brasil). Pages 271-283 in M. T. Melo, editor. A Primatologia no Brasil. SBPr, Belo Horizonte- Brazil.

Valle, Y. B. M., M. A. O. Monteiro da Cruz, L. C. O. Melo, and M. M. ValençaMontenegro. 2005. Análise comportamental de fêmeas sexualmente maduras em Callithrix jacchus de vida livre nos períodos pré- e pós-parto.in XI Congresso Brasileiro de Primatologia.

Vallinoto, M., L. Sena, I. Sampaio, H. Schneider, and M. P. Schneider. 2000. Mitochondrial DNA-like sequence in the nuclear genome of Saguinus (Callitrichinae, Primates):Transfer estimation. Genetics and Molecular Biology 23:35-42.

Van Roosmalen, M. G. M., T. Van Roosmalen, and R. A. mittermeier. 2002. A taxonomic review of the titi monkeys, genus Callicebus Thomas, 1903, with the description of two new species, Callicebus bernhardi and Callicebus stephennashi, from Brazilian Amazonia. Neotropical Primates 10:1-52.

Vaz, S. M. 2001. Primatas da região do Rio Tapajós, Pará, Brasil. Neotropical Primates 9:54-57.

Vaz, S. M. 2005. Mamíferos coletados em Pedra Branca, Município de Paraty, Rio de Janeiro, Brasil. Revista Brasileira de Zoologia 22:1164-1169.

Veras, M. M. 2004. Aspectos morfológicos do aparelho reprodutor feminino em bugios ( Alouatta guariba e Alouatta cararya) o modelo feminino.

Veras, M. M., M. A. Miglino, and Z. Silva. 2007. Morfologia do aparelho reprodutor em fêmeas de bugio marrom (Alouatta guariba clamitans). Brazilian Journal of Veterinary Research and Animal Science 44:12-17. 
Verderane, M. P., E. D. Ramos da Silva, N. Spagnoletti, D. M. Fragasy, E. Visalberghi, E. B. Ottoni, and P. Izar. 2007. Predation of vertebrates by wild capuchin monkeys (Cebus libidinosus) in a zone of ecological transition between Cerrado and Caatinga in Piaui.in XII Congresso Brasileiro de Primatologia.

Verissimo, K. C. S., L. Ianuzzi, and M. A. B. Oliveira. 2007. Dieta do Callithrix jacchus (Linnaeus, 1758) em ambiente de restinga do nordeste do Brasil.in XII Congresso Brasileiro de Primatologia.

Verissimo, K. C. S. and M. A. B. Oliveira. 2007. Callithrix jacchus (Linnaeus, 1758) na restinga: influência da distribuição espacial da vegetação e da sazonalidade sobre a área de uso.in XII Congresso Brasileiro de Primatologia.

Vilel, A. A. and K. Del Claro. 2007. Caracterização das populações e uso de habitat por Callithrix penicillata em uma reserva de cerrado no triângulo mineiro.in XII Congresso Brasileiro de Primatologia.

Vilela, S. L. 2007. Simpatria e dieta de Callithrix penicillata (Hershkovitz) (Callitrichidae) e Cebus libidinosus (Spix) (Cebidae) em matas de galeria do Distrito Federal, Brasil. Revista Brasileira de Zoologia 24:601-607.

Vilinec, K., J. E. M. Lambery, and J. David. 2006. Primate and dung beetle communities in secondary growth rain forests: implications for conservation of seed dispersal systems. International Journal of Primatology 27:855-879.

Villar, D. N. A., T. C. Costa, and F. D. C. Mendes. 2007. Population census of Cebus libidinosus in the Altamiro de Moura Pacheco State Park, an area of Cerrado in Goiania (GO).in XII Congresso Brasileiro de Primatologia.

Visalberghi, E., D. Fragaszy, E. Ottoni, P. Izar, M. G. de Oliveira, and F. R. D. Andrade. 2007. Characteristics of hammer stones and anvils used by wild bearded capuchin monkeys (Cebus libidinosus) to crack open palm nuts. American Journal of Physical Anthropology 132:426-444.

Vivo, M. d. 1991. Taxonomia de Callithrix Erxleben, 1777 (Callitrichidae, Primates). Fundação Biodiversitas, Belo Horizonte- Brazil.

Waga, I. C., P. S. Pinha, G. Sabbatini, M. Stammati, and M. C. H. Tavares. 2007. Predation and the ingestion of vertebrates by capuchin monkeys (Cebus libidinosus) in the Brasilia National Park.in XII Congresso Brasileiro de Primatologia.

Wallace, R. B., R. L. E. Painter, D. I. Rumiz, and A. B. Taber. 2000. Primate diversity, distribution and relative abundances in the Rio Blanco y Negro wildlife reserve, Santa Cruz, Bolivia. Neotropical Primates. 8:24-28.

Wright, B. W., K. A. Wright, J. Chalk, M. P. Verderane, D. Fragaszy, E. Visalberghi, P. Izar, E. B. Ottoni, P. Constantino, and C. Vinyard. 2009. Fallback foraging as a way of life: Using dietary toughness to compare the fallback signal among capuchins and implications for interpreting morphological variation. American Journal of Physical Anthropology 140:687-699.

Zago, L., A. L. Regolin, L. Juk, and C. V. Santos. 2007. Resultados preliminares de estimativa populacional e ecologia de Callithrix penicillata no parque ecológico do Córrego Grande (Florianópolis, SC).in XII Congresso Brasileiro de Primatologia. 